\title{
LNG Station Analysis for the Prediction of Pressure Rise and Vented Emissions
}

John T. Hailer

Follow this and additional works at: https://researchrepository.wvu.edu/etd

\section{Recommended Citation}

Hailer, John T., "LNG Station Analysis for the Prediction of Pressure Rise and Vented Emissions" (2015). Graduate Theses, Dissertations, and Problem Reports. 5735.

https://researchrepository.wvu.edu/etd/5735

This Thesis is protected by copyright and/or related rights. It has been brought to you by the The Research Repository @ WVU with permission from the rights-holder(s). You are free to use this Thesis in any way that is permitted by the copyright and related rights legislation that applies to your use. For other uses you must obtain permission from the rights-holder(s) directly, unless additional rights are indicated by a Creative Commons license in the record and/ or on the work itself. This Thesis has been accepted for inclusion in WVU Graduate Theses, Dissertations, and Problem Reports collection by an authorized administrator of The Research Repository @ WVU. For more information, please contact researchrepository@mail.wvu.edu. 


\title{
LNG Station Analysis for the Prediction of Pressure Rise and Vented Emissions
}

\author{
John T. Hailer \\ Thesis submitted \\ to the Benjamin M. Statler College of Engineering and Mineral Resources \\ at West Virginia University \\ in partial fulfillment of the requirements for the degree of \\ Master of Science in \\ Mechanical Engineering \\ Dr. Scott Wayne, Chair \\ Dr. Gregory Thompson \\ Dr. John Zondlo \\ Department of Mechanical and Aerospace Engineering \\ Morgantown, West Virginia \\ 2015
}

Keyword: LNG, Natural Gas, Emissions, Methane, Model, Heat Transfer Copyright 2015 John T. Hailer 


\title{
Abstract \\ LNG Station Analysis for the Prediction of Pressure Rise and Vented Emissions
}

\author{
John T. Hailer
}

Liquefied natural gas is seeing increased usage in the over-the-road trucking sector. Stations which refuel LNG vehicles sometimes emit natural gas into the atmosphere through a pressure relief valve due to over pressurization of the storage tank. Methane, the dominant constitute in natural gas, is considered a more potent greenhouse gas than carbon dioxide. However, natural gas has less of a carbon foot print than other fuels such as gasoline, diesel, and coal when burned giving it a global warming potential (GWP) advantage. Emission of methane into the atmosphere degrades the GWP advantage of burned methane or natural gas. Therein a tipping point exists where switching from conventional fuels to natural gas, if natural gas emissions are high, reduces or eliminates benefits in terms of GWP. This has many concerned about the accurate and comprehensive quantization of emissions from natural gas systems to enable well informed policy decisions for the natural gas sector.

A numerical model was developed to predict the pressurization of a LNG vehicle refueling station for the purpose of estimating vented emissions emanating from the pressure release valve (PRV). Factors which were addressed included the behavior of components of a station, local environmental conditions, and the vehicle fleet characteristics. The model formulation used two thermodynamic approaches to predict pressure rise. The thermodynamics were coupled with heat transfer calculated from station components including the bulk storage tank, transfer piping, and dispensers. Energy transfer was calculated by a combination of specific idealizations of cryogenic components and 1-D resistance networks incorporated into computer algorithms detailed in this thesis. Data were collected from two operating LNG stations, including the associated vehicle fleets, and were used for model creation and validation. Comparison of the pressure rise rate between the collected data and the model results indicated an average absolute model error for the first LNG station of $7.3 \%$ and for the second station of $25.1 \%$. A range of emissions was deduced from the data indicating the first station emitted $0.1 \%$ to $1.5 \%$ of fuel dispensed to vehicles and $0.9 \%$ to $5.3 \%$ for the second station over the course of the 3 week evaluation periods. The analysis of the stations indicated vehicle transactions were a major factor in the pressure trends of the bulk storage tank. Vehicles refueling at the station caused the pressure rise rate to increase driving PRV emissions. However, at a certain point, specific to the design of each station, removal of LNG by vehicles outpaced pressure rise which caused stations to have zero PRV emissions. Therefore, stations emissions found were indicative of stations tendency to venting but not representative of stations on the whole. 


\section{Acknowledgements}

I would like to thank my family for their unwavering support and encouragement which allowed me to pursue a master's degree. I also wish to thank Dr. Scott Wayne for his patience, generosity, and advice during my education at WVU. As well, I thank Dr. Gregory Thompson and Dr. John Zondlo for their support and advice in writing this thesis. I extend my thanks to my coworker Cesar Sandoval who put a great deal of time and thought into the thermodynamic models used in conjunction with this thesis. Much of the data presented was collected or negotiated for through the hard work and effort of Dr. Nigel Clark, David McKain, April Covington, and Dr. Derek Johnson in support of the research study. I am grateful for the opportunity of working with WVU CAFEE.

In addition, support was provided by the Environmental Defense Fund (EDF), Cummins, Cummins-Westport, Royal Dutch Shell, the American Gas Association, Chart Industries, Clean Energy, the International Council on Clean Transportation, PepsiCo, Volvo Group, Waste Management, and Westport Innovations. Funding for EDF's methane research series, including the West Virginia University study, was provided for by Fiona and Stan Druckenmiller, Heising-Simons Foundation, Bill and Susan Oberndorf, Betsy and Sam Reeves, Robertson Foundation, Alfred P. Sloan Foundation, TomKat Charitable Trust, and the Walton Family Foundation. 


\section{Contents}

LNG Station Analysis for the Prediction of Pressure Rise and Vented Emissions..................................... i

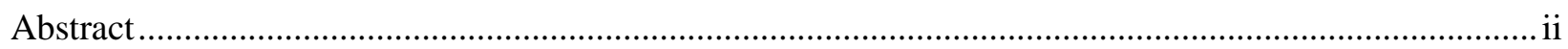

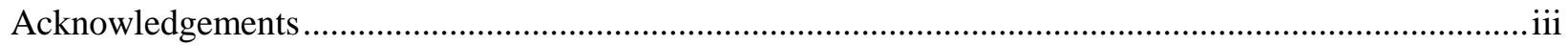

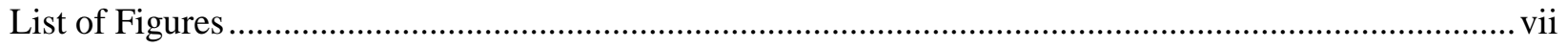

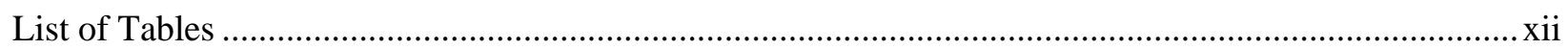

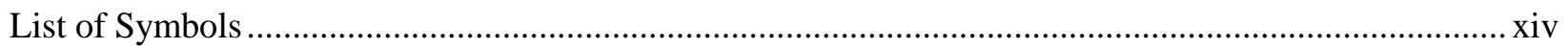

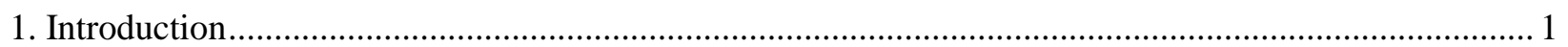

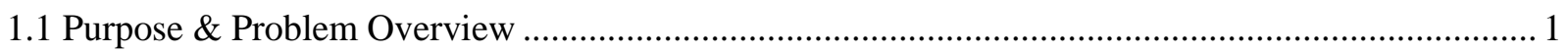

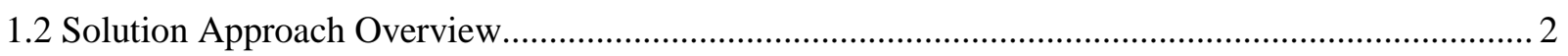

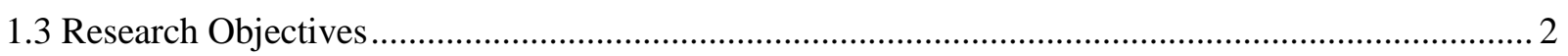

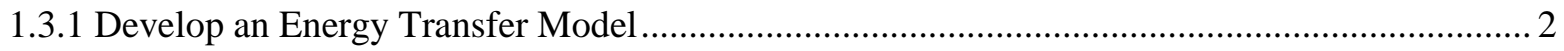

1.3.2 Incorporate Energy Transfer Model into a Larger Process Model............................................. 3

1.3.3 Compare Larger Process Model against Experimental Data .................................................... 3

1.4 Project Scope ….................................................................................................................

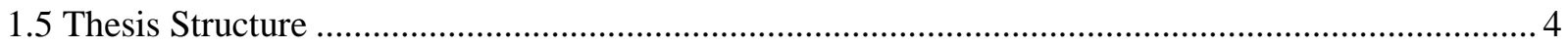

2. Background \& Literature Review ................................................................................................... 5

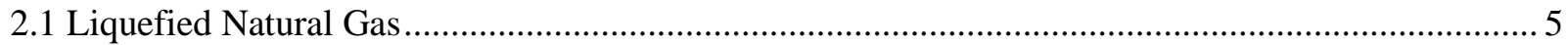

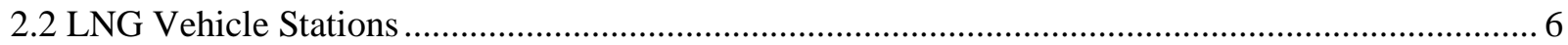

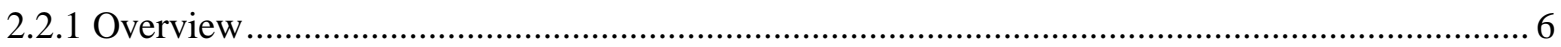

2.2.2 LNG Station Vehicle Fleet................................................................................................... 7

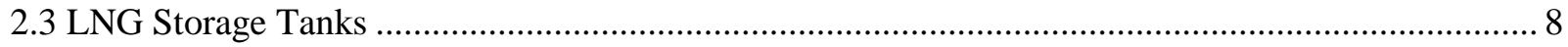

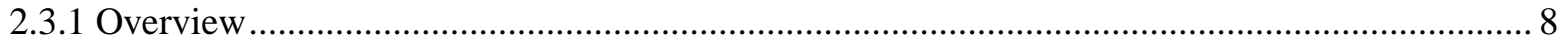

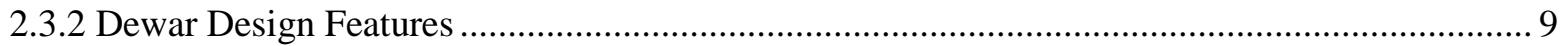

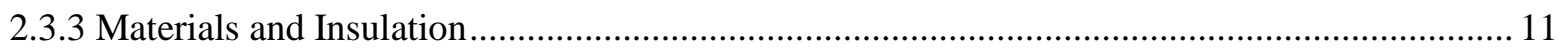

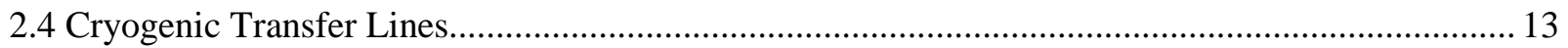

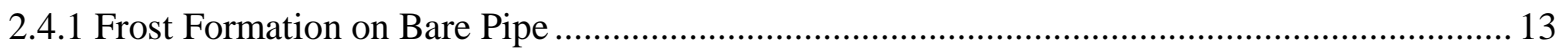

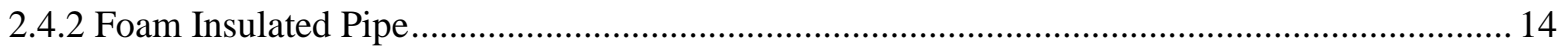

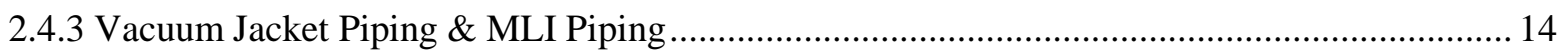

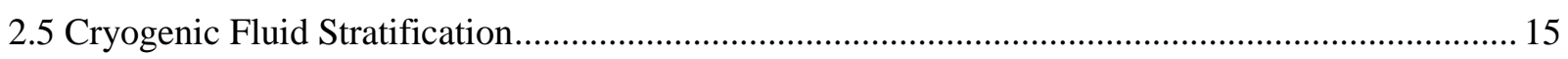

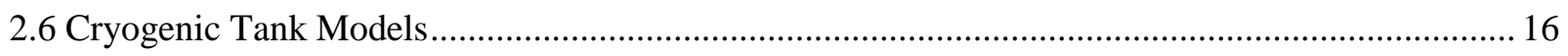

2.6.1 Additional Model Research Information ......................................................................... 17

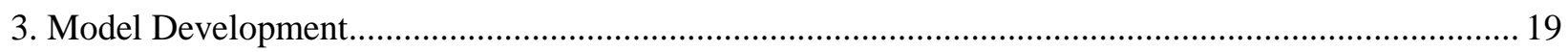


3.1 LNG Station Energy Transfer Model Development Introduction ............................................... 19

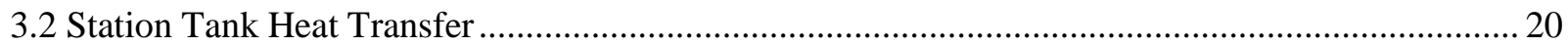

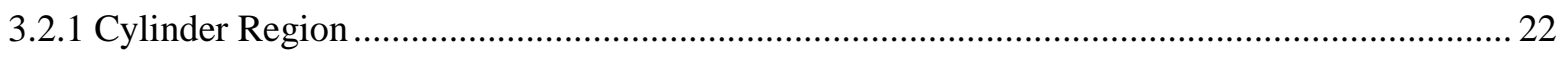

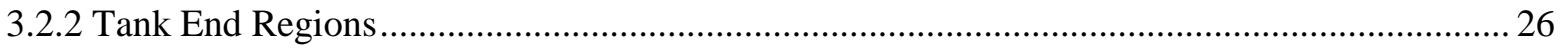

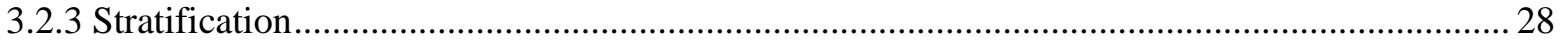

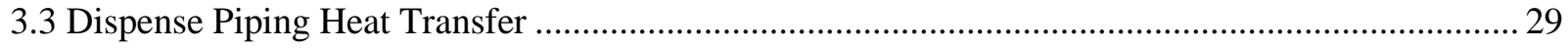

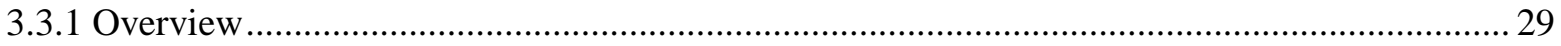

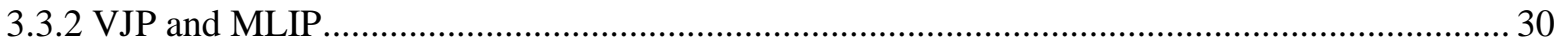

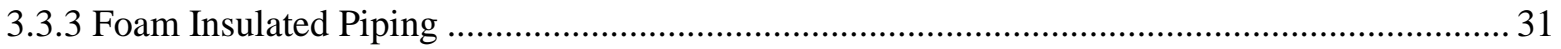

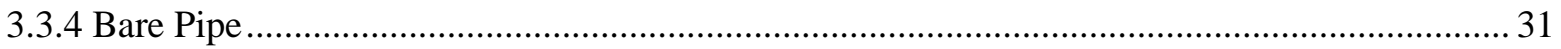

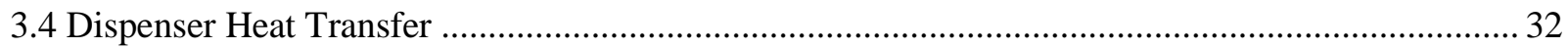

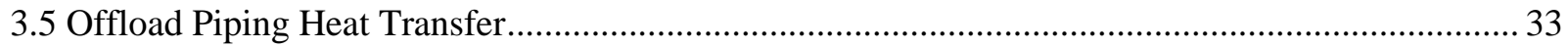

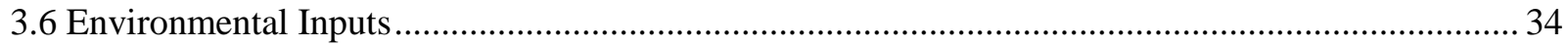

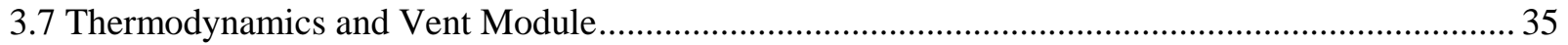

3.7.1 Saturated Homogeneous Thermodynamic Approach ............................................................. 35

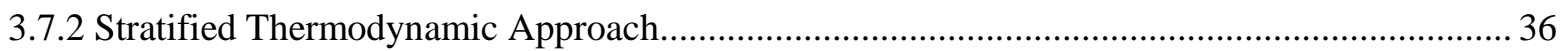

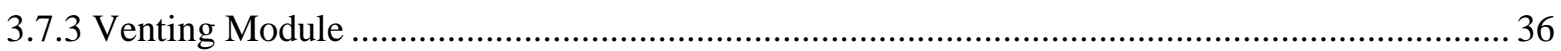

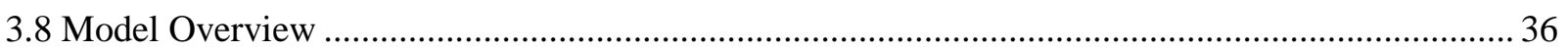

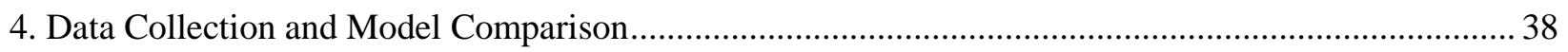

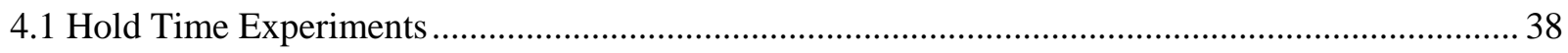

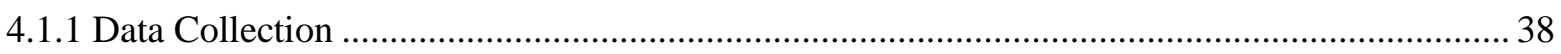

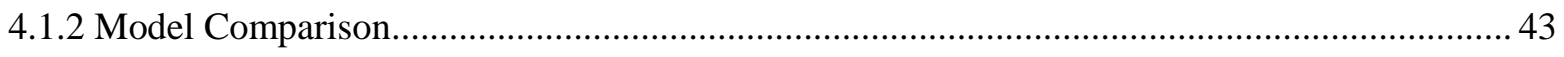

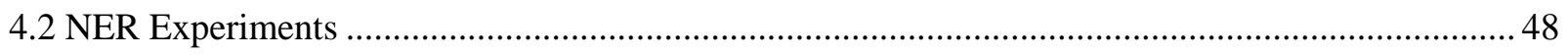

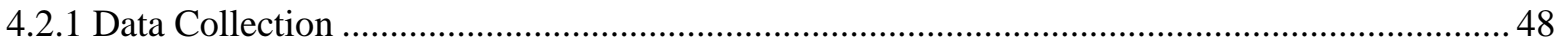

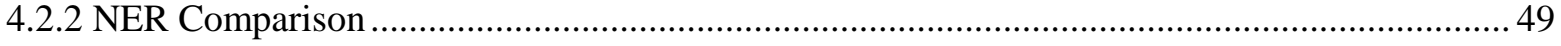

4.3 Full Station Tank Model Experiments ....................................................................................... 51

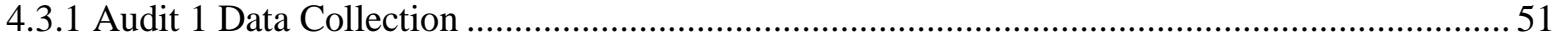

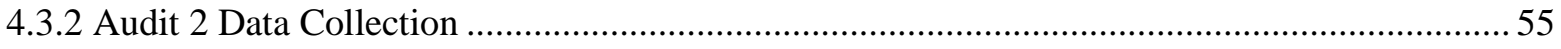

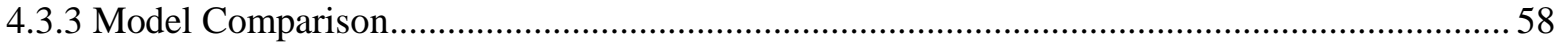

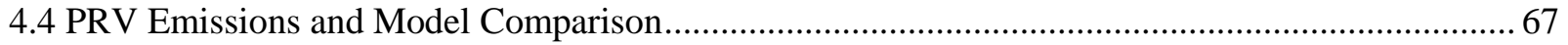

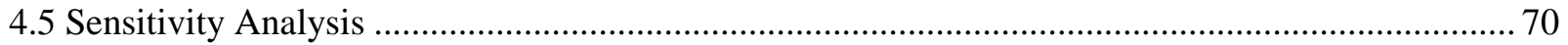

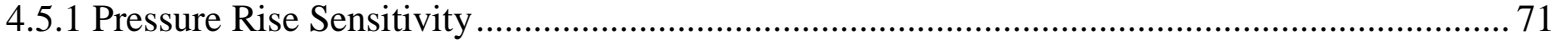

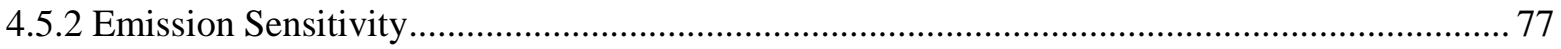

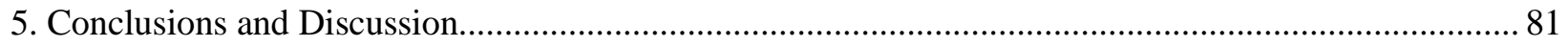




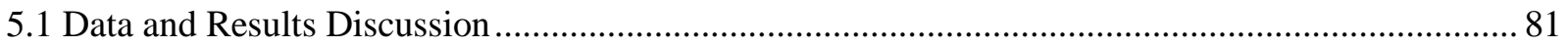

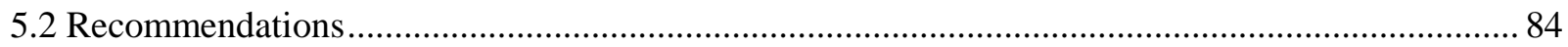

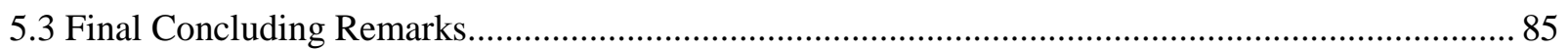

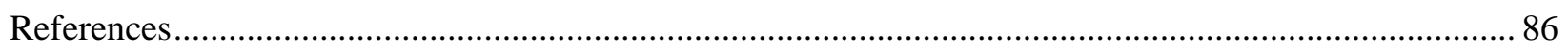

A- Appendix: Additional Model Development Information ................................................................ A-1

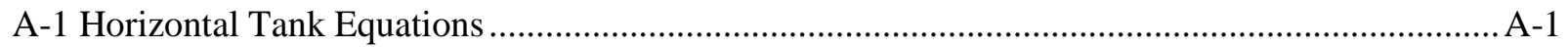

A-2 Station Tank Area Ratio Calculation .................................................................................... A

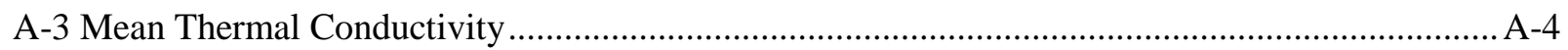

B- Appendix B: Additional Data Collection Information...................................................................... B-1

B-1 Vehicle Tank Sensor Accuracy .......................................................................................... B-1

B-2 Vehicle Tank NER Sensor Accuracy .................................................................................... B-3

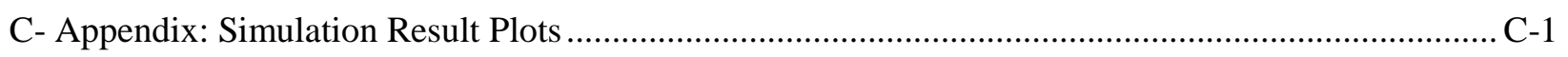

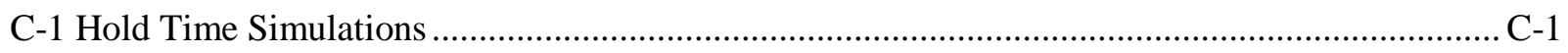

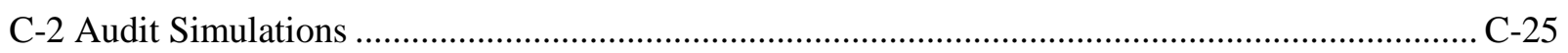




\section{List of Figures}

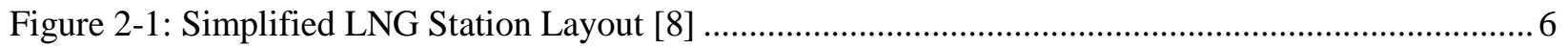

Figure 2-2: Flat Bottomed Large Cryogenic LNG Storage Tanks [9], [10] ........................................... 8

Figure 2-3: Horizontal and Vertical LNG Storage Tanks for Vehicle Fueling Facilities [11], [12]............ 8

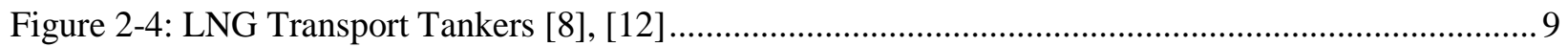

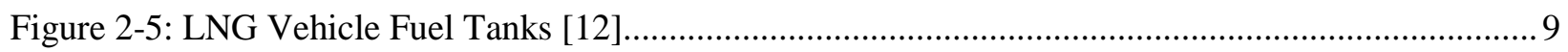

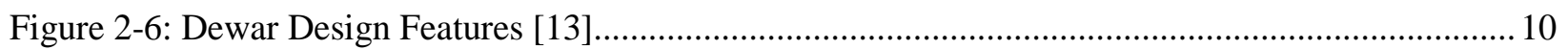

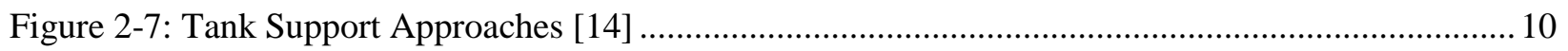

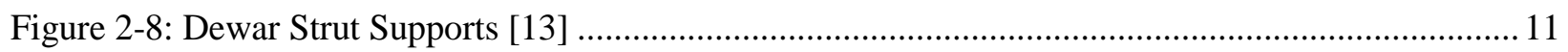

Figure 2-9: Thermal Conductivity of Stainless Steel 304 from 4K to 300K [18]................................... 13

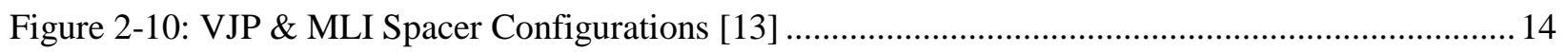

Figure 2-11: Convection in an Enclosed Tank [25] ............................................................................. 15

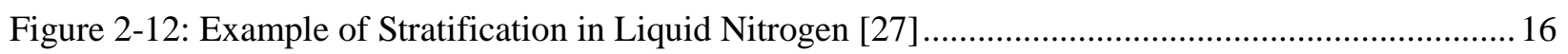

Figure 3-1: Station Components Modeled for Energy Transfer to Storage Tank LNG............................ 19

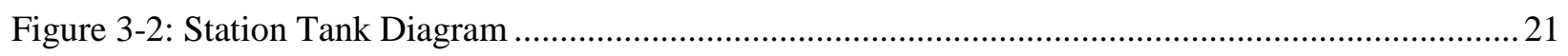

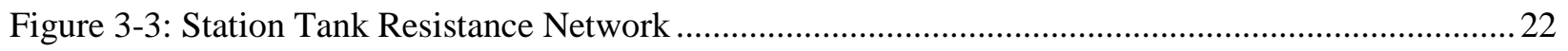

Figure 3-4: Illustration of Thermal Stratification Layers for Model....................................................... 28

Figure 3-5: MLIP Diagram \& Thermal Resistance Network...................................................................... 30

Figure 3-6: Assumed Daily Solar Loading Profile for Audit Station Comparions.................................... 34

Figure 3-7: LNG Station Model Flow Chart........................................................................................... 37

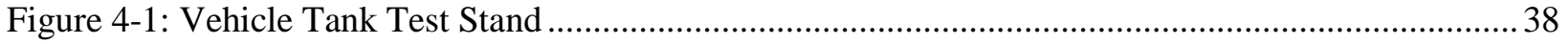

Figure 4-2: NON-IFP Tank Hold Time Test 2 - Tank Pressure Rise Trend with PRV Historesis ............. 39

Figure 4-3: NON-IFP Tank Hold Time Test 2 - Tank Weight Used to Calculate Fill Level..................... 40

Figure 4-4: NON-IFP Tank Hold Time Test 2 - Ambient Temperatures and Surface Temperatures for

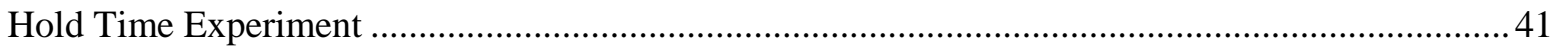

Figure 4-5: NON-IFP Tank Hold Tim Test 2 - Solar Loading Incident on Tank Surfaces....................... 41

Figure 4-6: Exp. 4 NON-IFP Tank Hold Time Sim. - Tank Heat Distribution..........................................47

Figure 4-7: NON-IFP Tank NER Test 1 - Heat Leak Extraplated from Normal Evaporation Rate by Using

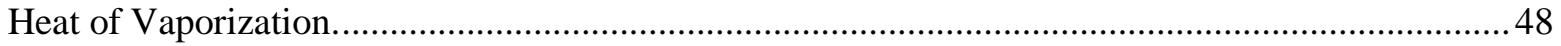

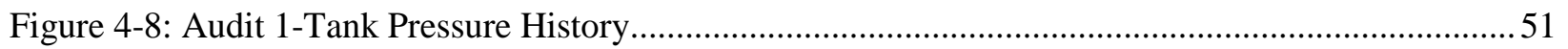

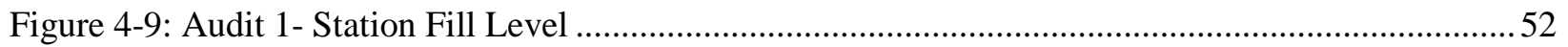

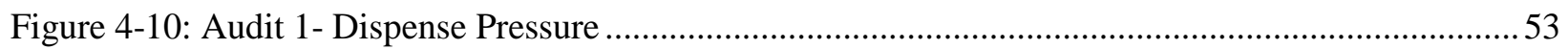

Figure 4-11: Audit 1- Offload Pump Pressure and Temperature .............................................................. 53

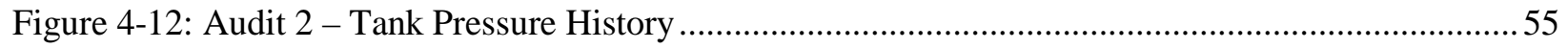


Figure 4-13: Audit 2 Segment 4 Simulation Pressure Comparison ......................................................... 59

Figure 4-14: Audit 2 Segment 6 Simulation Pressure Comparison ......................................................... 60

Figure 4-15: Audit 1 Pressure Rise Rate Comparison ................................................................................ 61

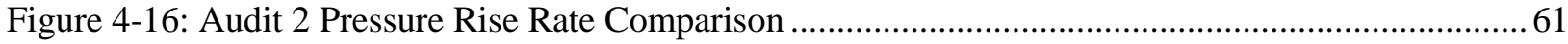

Figure 4-17: Audit 1 Average Energy Transfer Rate for Each Segment by Source ................................... 63

Figure 4-18: Audit 2 Average Energy Transfer Rate for Each Segment by Source .................................. 63

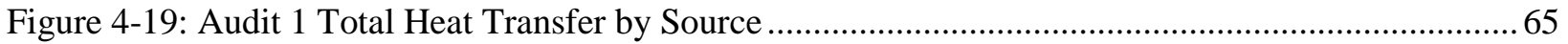

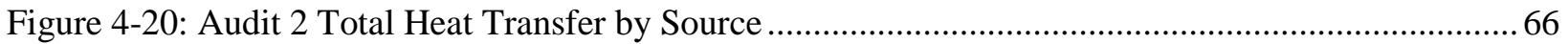

Figure 4-21: Heat Transfer by Source Over All Audit 1 Segments .......................................................... 67

Figure 4-22: Heat Transfer by Source Over All Audit 2 Segments ........................................................6 67

Figure 4-23: Audit 1 Segment 3 - Pressure Rise History with Venting Module Behavior ........................ 69

Figure 4-24: Audit 1 Segment 4 - Pressure Rise History with Venting Module Behavior ......................... 70

Figure 4-25: Audit 1 Seg. 1 - Tank Pressure Rise Sensitivity to Ambient Temperature Modified by a

Precentage Decrease and Increase of the Average......................................................................... 71

Figure 4-26: Audit 1 Seg. 1 - Sensitivity to MLI Thermal Conductivity …............................................... 73

Figure 4-27: Audit 1 Seg. 1 -Variation of Dispense Piping Lengths Summary ........................................ 74

Figure 4-28: Audit 1 Seg. 1 - Sensitivity of Ambient Temperature............................................................. 75

Figure 4-29: Audit 1 Seg. 1 -Sensitivity to MLI Thermal Conductivity ................................................... 76

Figure 4-30: Audit 1 Seg. 3 - Dispense Piping Lengths Venting Module Sensitivity Pressure Trends..... 78

Figure A-1: Horizontal Tank Stratification Layers and Geometry ....................................................... A-2

Figure B-1: NON-IFP LNG Tank Attached to Data Acquisition System............................................... B-2

Figure B-2: IFP LNG Tank Attached to Data Acquisition System ..................................................... B-2

Figure B-3: Full Flow Sampling System ....................................................................................... B-3

Figure C-1: NON-IFP Tank Exp. 2 Hold Time Simulation Pressure Comparison.................................... C-1

Figure C-2: NON-IFP Tank Exp. 2 Hold Time Simulation Stratified Tank Heat Transfer...................... C-1

Figure C-3: NON-IFP Tank Exp. 2 Hold Time Simulation Ambient Temperature ................................. C-2

Figure C-4: NON-IFP Tank Exp. 2 Hold Time Simulation Solar Loading ............................................ C-2

Figure C-5: NON-IFP Tank Exp. 3 Hold Time Simulation Pressure Comparison....................................

Figure C-6: NON-IFP Tank Exp. 3 Hold Time Simulation Stratified Tank Heat Transfer.......................

Figure C-7: NON-IFP Tank Exp. 3 Hold Time Simulation Ambient Temperature ..................................

Figure C-8: NON-IFP Tank Exp. 3 Hold Time Simulation Solar Loading ..............................................-4

Figure C-9: NON-IFP Tank Exp. 4 Hold Time Simulation Pressure Comparison................................... C-5

Figure C-10: NON-IFP Tank Exp. 4 Hold Time Simulation Stratified Tank Heat Transfer.................... C-5

Figure C-11: NON-IFP Tank Exp. 4 Hold Time Simulation Ambient Temperature .................................6 
Figure C-12: NON-IFP Tank Exp. 4 Hold Time Simulation Solar Loading ............................................6

Figure C-13: NON-IFP Tank Exp. 5 Hold Time Simulation Pressure Comparison ................................. C-7

Figure C-14: NON-IFP Tank Exp. 5 Hold Time Simulation Stratified Tank Heat Transfer.....................-7

Figure C-15: NON-IFP Tank Exp. 5 Hold Time Simulation Ambient Temperature ................................8

Figure C-16: NON-IFP Tank Exp. 5 Hold Time Simulation Tank Heat Transfer..................................... C-8

Figure C-17: NON-IFP Tank Exp. 6 Hold Time Simulation Pressure Comparison................................. C-9

Figure C-18: NON-IFP Tank Exp. 6 Hold Time Simulation Tank Heat Transfer................................... C-9

Figure C-19: NON-IFP Tank Exp. 6 Hold Time Simulation Ambient Temperature ............................. C-10

Figure C-20: NON-IFP Tank Exp. 6 Hold Time Simulation Tank Heat Transfer................................. C-10

Figure C-21: NON-IFP Tank Exp. 7 Hold Time Simulation Pressure Comparison............................... C-11

Figure C-22: NON-IFP Tank Exp. 7 Hold Time Simulation Tank Heat Transfer.................................. C-11

Figure C-23: NON-IFP Tank Exp. 7 Hold Time Simulation Ambient Temperature ............................. C-12

Figure C-24: NON-IFP Tank Exp. 7 Hold Time Simulation Tank Heat Transfer.................................. C-12

Figure C-25: NON-IFP Tank Exp. 8 Hold Time Simulation Pressure Comparison............................... C-13

Figure C-26: NON-IFP Tank Exp. 8 Hold Time Simulation Tank Heat Transfer.................................. C-13

Figure C-27: NON-IFP Tank Exp. 8 Hold Time Simulation Ambient Temperature ...............................14

Figure C-28: NON-IFP Tank Exp. 8 Hold Time Simulation Tank Heat Transfer....................................14

Figure C-29: NON-IFP Tank Exp. 9 Hold Time Simulation Pressure Comparison.................................15

Figure C-30: NON-IFP Tank Exp. 9 Hold Time Simulation Tank Heat Transfer...................................15

Figure C-31: NON-IFP Tank Exp. 9 Hold Time Simulation Ambient Temperature ..............................16

Figure C-32: NON-IFP Tank Exp. 9 Hold Time Simulation Tank Heat Transfer...................................16

Figure C-33: IFP Tank Exp. 2 Hold Time Simulation Pressure Comparison..........................................17

Figure C-34: IFP Tank Exp. 2 Hold Time Simulation Tank Heat Transfer............................................17

Figure C-35: IFP Tank Exp. 2 Hold Time Simulation Ambient Temperature ........................................ C-18

Figure C-36: IFP Tank Exp. 2 Hold Time Simulation Tank Heat Transfer............................................ C-18

Figure C-37: IFP Tank Exp. 3 Hold Time Simulation Pressure Comparison........................................ C-19

Figure C-38: IFP Tank Exp. 3 Hold Time Simulation Tank Heat Transfer............................................ C-19

Figure C-39: IFP Tank Exp. 3 Hold Time Simulation Ambient Temperature .......................................20

Figure C-40: IFP Tank Exp. 3 Hold Time Simulation Tank Heat Transfer........................................... C-20

Figure C-41: IFP Tank Exp. 4 Hold Time Simulation Pressure Comparison......................................... C-21

Figure C-42: IFP Tank Exp. 4 Hold Time Simulation Tank Heat Transfer............................................21

Figure C-43: IFP Tank Exp. 4 Hold Time Simulation Ambient Temperature .......................................22

Figure C-44: IFP Tank Exp. 4 Hold Time Simulation Tank Heat Transfer........................................... C-22

Figure C-45: IFP Tank Exp. 5 Hold Time Simulation Pressure Comparison........................................ C-23 
Figure C-46: IFP Tank Exp. 5 Hold Time Simulation Tank Heat Transfer.. C-23

Figure C-47: IFP Tank Exp. 5 Hold Time Simulation Ambient Temperature C-24

Figure C-48: IFP Tank Exp. 5 Hold Time Simulation Tank Heat Transfer..... C-24

Figure C-49: Audit 1 Segment 1 Simulation Pressure Comparison. C-25

Figure C-50: Audit 1 Segment 1 Simulation Heat Transfer C-25

Figure C-51: Audit 1 Segment 1 Ambient Temperature. C-26

Figure C-52: Audit 1 Segment 1 Simulation Fleet Arrival Times and Mass Dispensed C-26

Figure C-53: Audit 1 Segment 2 Simulation Pressure Comparison. C-27

Figure C-54: Audit 1 Segment 2 Simulation Heat Transfer $\mathrm{C}-27$

Figure C-55: Audit 1 Segment 2 Simulation Fleet Arrival Times and Mass Dispensed C-28

Figure C-56: Audit 1 Segment 2 Ambient Temperature. C-28

Figure C-57: Audit 1 Segment 3 Simulation Pressure Comparison. C-29

Figure C-58: Audit 1 Segment 3 Simulation Heat Transfer C-29

Figure C-59: Audit 1 Segment 3 Ambient Temperature. C-30

Figure C-60: Audit 1 Segment 3 Simulation Fleet Arrival Times and Mass Dispensed C-30 Figure C-61: Audit 1 Segment 4 Simulation Pressure Comparison. C-31 Figure C-62: Audit 1 Segment 4 Simulation Heat Transfer C-31 Figure C-63: Audit 1 Segment 4 Ambient Temperature. C-32 Figure C-64: Audit 1 Segment 4 Simulation Fleet Arrival Times and Mass Dispensed .........................32 Figure C-65: Audit 1 Segment 5 Simulation Pressure Comparison......................................................... C-33 Figure C-66: Audit 1 Segment 5 Simulation Heat Transfer .................................................................33

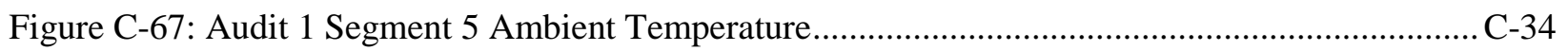
Figure C-68: Audit 1 Segment 5 Simulation Fleet Arrival Times and Mass Dispensed ........................ C-34 Figure C-69: Audit 1 Segment 6 Simulation Pressure Comparison.........................................................35 Figure C-70: Audit 1 Segment 6 Simulation Heat Transfer ...................................................................35 Figure C-71: Audit 1 Segment 6 Ambient Temperature........................................................................ C-36 Figure C-72: Audit 1 Segment 6 Simulation Fleet Arrival Times and Mass Dispensed ....................... C-36

Figure C-73: Audit 2 Segment 1 Simulation Pressure Comparison.........................................................37

Figure C-74: Audit 2 Segment 1 Simulation Heat Transfer ....................................................................37

Figure C-75: Audit 2 Segment 1 Ambient Temperature..........................................................................38

Figure C-76: Audit 2 Segment 1 Simulation Fleet Arrival Times and Mass Dispensed ........................ C-38

Figure C-77: Audit 2 Segment 2 Simulation Pressure Comparison........................................................ C-39

Figure C-78: Audit 2 Segment 2 Simulation Heat Transfer ..................................................................39

Figure C-79: Audit 2 Segment 2 Ambient Temperature........................................................................ 
Figure C-80: Audit 2 Segment 2 Simulation Fleet Arrival Times and Mass Dispensed ........................ C-40

Figure C-81: Audit 2 Segment 3 Simulation Pressure Comparison...................................................... C-41

Figure C-82: Audit 2 Segment 3 Simulation Heat Transfer ............................................................... C-41

Figure C-83: Audit 2 Segment 3 Simulation Fleet Arrival Times and Mass Dispensed ........................ C-42

Figure C-84: Audit 2 Segment 3 Ambient Temperature.................................................................... C-42

Figure C-85: Audit 2 Segment 4 Simulation Pressure Comparison........................................................43

Figure C-86: Audit 2 Segment 4 Simulation Heat Transfer ..................................................................43

Figure C-87: Audit 2 Segment 4 Ambient Temperature....................................................................

Figure C-88: Audit 2 Segment 4 Simulation Fleet Arrival Times and Mass Dispensed ......................... C-44

Figure C-89: Audit 2 Segment 5 Simulation Pressure Comparison.......................................................45

Figure C-90: Audit 2 Segment 5 Simulation Heat Transfer .................................................................45

Figure C-91: Audit 2 Segment 5 Ambient Temperature..........................................................................

Figure C-92: Audit 2 Segment 5 Simulation Fleet Arrival Times and Mass Dispensed ......................... C-46

Figure C-93: Audit 2 Segment 6 Simulation Pressure Comparison.......................................................47

Figure C-94: Audit 2 Segment 6 Simulation Heat Transfer ...............................................................4

Figure C-95: Audit 2 Segment 6 Simulation Fleet Arrival Times and Mass Dispensed .........................-48

Figure C-96: Audit 2 Segment 6 Ambient Temperature........................................................................

Figure C-97: Audit 2 Segment 7 Simulation Pressure Comparison.........................................................49

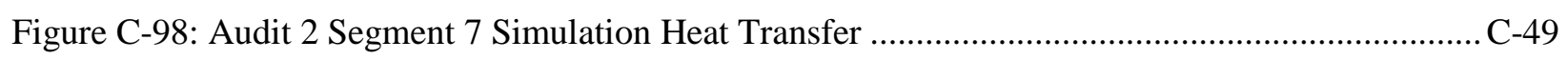

Figure C-99: Audit 2 Segment 7 Simulation Fleet Arrival Times and Mass Dispensed ........................ C-50

Figure C-100: Audit 2 Segment 7 Ambient Temperature................................................................... -50

Figure C-101: Audit 2 Segment 8 Simulation Pressure Comparison ....................................................51

Figure C-102: Audit 2 Segment 8 Simulation Heat Transfer ............................................................

Figure C-103: Audit 2 Segment 8 Simulation Fleet Arrival Times and Mass Dispensed ....................... C-52

Figure C-104: Audit 2 Segment 8 Ambient Temperature................................................................. -52 


\section{List of Tables}

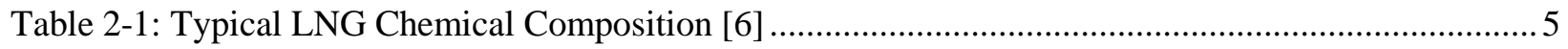

Table 2-2: Properties of Saturated Methane at $101.3 \mathrm{kPa}$. [5] .................................................................. 5

Table 2-3: Properties of Evacuated Powders and Fibrous Insulation Materials. [13]............................... 12

Table 2-4: Mean Thermal Conductivity of MLI in Vacuum at Various Boundry Temperatures [16] ........ 12

Table 3-1: Common Surface Absorptivity and Emissivity Values [47] ................................................... 25

Table 3-2: MLIP Specifications From Industry Technical Specifications [50], [52] ............................... 31

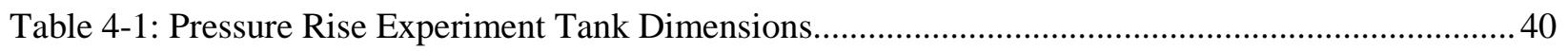

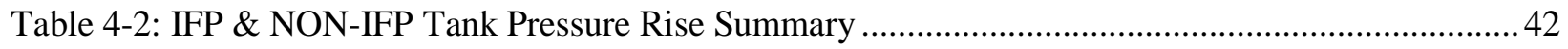

Table 4-3: Tank Hold Time Pressure Rise Rate Comparison .................................................................. 43

Table 4-4: Tank Hold Time Final Pressure Comparison ...................................................................... 44

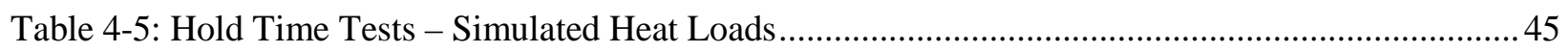

Table 4-6: Hold Time Simulations - Average Heat Leak to Liquid and Vapor Phases ............................. 46

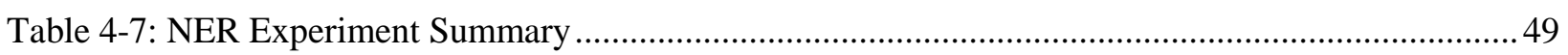

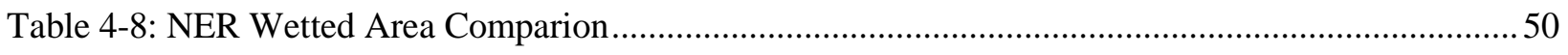

Table 4-9: Audit 1 Vehicle Transaction Summary .................................................................................. 54

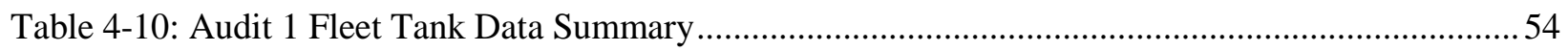

Table 4-11: Audit 1 Experimental Data Summary by Segment ............................................................... 55

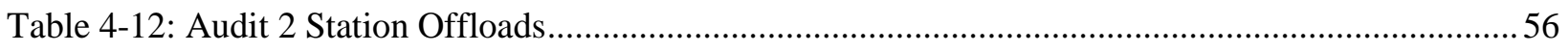

Table 4-13: Audit 2 Manual Station Vent (Witnessed) ............................................................................ 56

Table 4-14: Audit 2 Vehicle Transaction Summary ............................................................................... 57

Table 4-15: Audit 2 Fleet Tank Data Summary ......................................................................................... 57

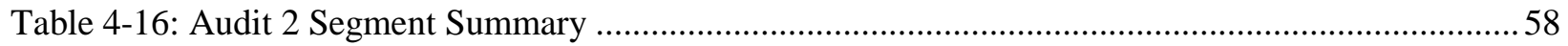

Table 4-17: Audit Pressure Rise Rate and Model Comparison .............................................................. 58

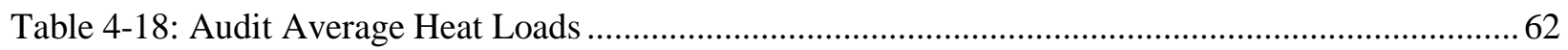

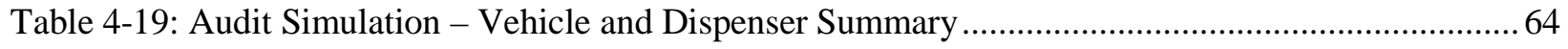

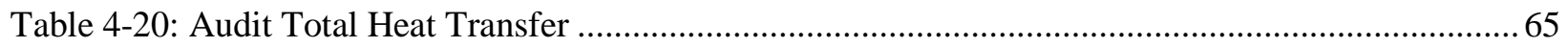

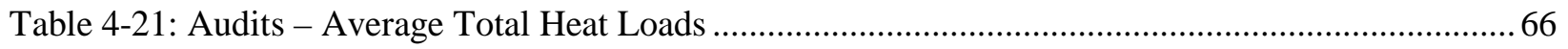

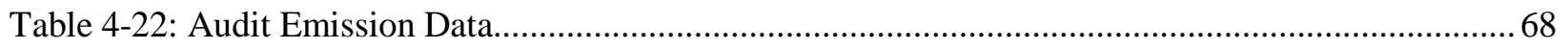

Table 4-23: Full Station Model Emission Prediction for Audit 1 _.............................................................69

Table 4-24: Audit 1 Seg. 1 - Percent Variation of Ambient Temperature Sensitivity Summary................72

Table 4-25: Audit 1 Seg. 1 - Percent Variation of MLI Thermal Conductivity Summary ........................ 73

Table 4-26: Audit 1 Seg. 1 -Variation of Dispense Piping Lengths Summary ......................................... 74

Table 4-27: Audit 1 Seg. 1 - Variation of Ambient Temperature Summary ............................................. 76 
Table 4-28: Audit 1 Seg. 1 -Sensitivity Study Variation of MLI Thermal Conductivity Summary .......... 77

Table 4-29: Dispense Piping Lenghts Emission Sensitivity Summary ..................................................... 79

Table 4-30: Thermal Conductivity of MLI Emission Sensitivity Summary............................................. 79

Table 4-31: Average Ambient Temperature Emission Sensitivity Summary ............................................ 79

Table 4-32: Vent Model Pressure Drop Emission Sensitivty Summary .................................................... 80

Table A-1: Acclerations Loads for Cryogenic Fluid Storage Vessel Suspension System........................A-3

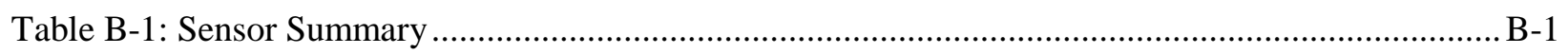




\section{List of Symbols}

$\alpha_{\mathrm{s}}-$ Absorptivity

$\mathrm{T}_{\mathrm{amb}}$ - Ambient temperature

$\gamma$ - Area ratio

$\mathrm{Cp}$ - Constant pressure specific heat

$\mathrm{Cv}$ - Constant volume specific heat

$\eta$-Convection weighting factor

$\mathrm{h}$ - Convection coefficient

$\mathrm{H}_{\text {vap }}$ - Heat of vaporization

$\mathrm{x}_{\mathrm{C}}-$ Critical length

$\rho$ - Density

D - Diameter

$\mu$ - Dynamic viscosity

$\varepsilon$ - Emissivity

$\mathrm{R}_{\mathrm{eq}}-$ Equivalent resistance

$\phi$ - Fill angle

$\mathrm{f}$ - Fill level

$\mathrm{Gr}$ - Grasholf number

$\dot{Q}-$ Heat transfer rate

$v$ - Kinematic viscosity

L- Length/Height

$\mathrm{L}_{\mathrm{f}}$ - Length fraction $\dot{m}$ - Mass flow rate

$\mathrm{k}_{\mathrm{m}}$ - Mean thermal conductivity

$\mathrm{Nu}$ - Nusselt number

Pr - Prandtl number

$\mathrm{P}$ - Pressure

$\mathrm{r}$ - Radius

Ra - Rayleigh number

Re - Reynolds number

$\mathrm{P}_{\text {sat }}$ - Saturation pressure

$\theta$ - Solar angle

$G_{\text {solar }}$ - Solar loading

$\sigma$-Stefan-Boltzmann constant

$\mathrm{T}$ - Temperature

$\mathrm{k}$ - Thermal conductivity

$\alpha$ - Thermal diffusivity

$\beta$ - Thermal expansion coefficient

$\mathrm{R}$ - Thermal resistance

$\mathrm{t}$ - Time

dt - Time step

$U_{\infty}$ - Wind speed 


\section{Introduction}

\subsection{Purpose \& Problem Overview}

Liquefied natural gas (LNG) is a hydrocarbon fuel which is becoming a wider spread transportation fuel, especially in long haul over-the-road trucking. Methane, LNG's main constitute, is considered a potent greenhouse gas and as such has many concerned over the environmental impact from emissions [1]. Methane has a greater warming effect than carbon dioxide when present in the atmosphere, but since it decomposes into carbon dioxide naturally, any radiative forcing from methane eventually reduces to that of carbon dioxide. Therefore, methane has a variable global warming potential (GWP) depending upon what time frame is used (typically 20 yr. or 100 yr.) [1]. When burned as a fuel, methane produces less carbon dioxide per unit energy than what is associated with burning gasoline, diesel, or coal; giving it a GWP advantage when used as a fuel. In order to realize benefits from methane, emissions from existing and future infrastructure must be low enough to not counteract methane's GWP advantage. Estimates of methane emission rates from industry infrastructure were found to be lacking [1]. Consequently, the lack of data spurred this research [2].

West Virginia University's (WVU) Center for Alternative Fuels, Engines, and Emissions (CAFEE), the Environmental Defense Fund (EDF), and natural gas transportation leaders cooperated to begin quantifying pump-to-wheels natural gas emissions in the heavy duty transportation sector [3],[2]. The study looked at emissions from sources throughout the sector, including compressed natural gas (CNG) vehicles and stations as well as LNG vehicles and stations. For vehicles this included emissions associated with operation of the vehicle such as tail pipe emission, crankcase emissions, and blow down from tanks. At stations, components such as compressors, refueling nozzles, and pressure relief valves were investigated. In addition, an industry outlook was performed with emission estimates based on expected technology advancements and practice changes associated with the study. Much of the cumulative information from the study can be found in [3].

As LNG is a cryogenic fuel, with a boiling point of $111.7 \mathrm{~K}$ at $101.3 \mathrm{kPa}$, specific specialized storage systems and technology must be used to contain the fuel for extended periods of time. For vehicles and refueling stations alike low pressure cryogenic dewars are used for storage. Some energy from the warmer outside environment inevitably reaches the LNG stored in the dewar. The energy input increases the temperature and the pressure of the fluid inside the tank. Most LNG tanks do not have a refrigeration loop that removes energy from the vessel, therefore, the pressure in the vessel will steadily rise unless there is sufficient usage of the LNG. It is not practical for the tank to contain the vapor pressure beyond a limit, 
thus a relief system must vent LNG when the pressure exceeds a predetermined value. LNG may be filled into a tank at a temperature that corresponds to a working pressure far below the relief valve setting (690 $\mathrm{kPa}$ to $1380 \mathrm{kPa}$ ), which will suppress venting for a period of time. However, tank conditions can and do eventually reach a point where venting occurs. This thesis work was completed to determine the drivers for pressurization of an LNG fueling station, and estimate over pressurization venting emissions from the station's pressure relief valve.

\subsection{Solution Approach Overview}

Vented emissions from the pressure release valve (PRV) of a station tank presented challenges for direct measurement. The vent stack where the natural gas was released through was difficult to access being mounted above the storage tanks. Emissions from the tanks were concentrated and in large enough quantities to overwhelm most sensors. In addition, there was limitation in what could be done for measurement since exposing electronics to the potentially explosive vapor constituted a significant safety concern. Therefore, the method selected was to create a computer model of the station.

Pressure rise of a modern LNG storage vessel was observed to take days to weeks, depending on conditions, before venting occurred. The long time periods limited the availability of data and acquisition from actual sources. Since an empirical model was precluded due to the limited obtainable data, a numerical process model based on fundamental thermodynamic and heat transfer engineering theory was chosen. The process model was created in the MATLAB/Simulink program. Two thermodynamic approaches were used to calculate pressure rise in the cryogenic tank. The first approach assumed a thermodynamically saturated and homogeneous condition of the stored fluid. The second approach used convection theory to calculate thermal stratification inside the tank and then determine the state of the LNG. Both approaches were developed by Sandoval [4]. These thermodynamic approaches were driven by the energy input from components of the station to the LNG contained in the bulk storage tank. Energy transfer into the LNG was modeled by using 1-D heat transfer resistance networks but where necessary other approaches were also utilized. Algorithms were developed for the various heat transfer sources and linked together to calculate an overall energy input into the LNG. The development of the heat transfer model is presented in this thesis.

\subsection{Research Objectives}

\subsubsection{Develop an Energy Transfer Model}

The primary goal of this thesis is to develop a model which simulates and calculates the various heat transfer and energy inputs into the bulk storage tank at a LNG station based on the information available. In past literature few components of the station were considered, usually limited to heat transfer 
through the station tank walls, when discussing the driving forces behind the pressure rise in the station. However from discussions with station owners and operators other sources of energy input exist which drive pressure rise, and therefore emissions.

\subsubsection{Incorporate Energy Transfer Model into a Larger Process Model}

The next objective is to incorporate the energy transfer model into the larger process model for predicting pressure rise and venting emissions from LNG stations. The pressure rise in the station is handled by two thermodynamic approaches briefly described later. These were developed by Sandoval [4]. The energy transfer model provides the calculated energy input to the stored cryogenic fluid and the thermodynamic approaches use the energy input to determine the new state (pressure, temperature, etc.) inside the tank. In addition, the full process model simulates vehicle fueling and venting emissions from the station pressure release valve.

Information was collected from two operational stations in two separate audits which included detailed information about the station and associated vehicle fleets. In addition, experiments were performed on LNG vehicle tanks at WVU over the course of the study. Data from the station investigations, such as fuel transaction histories, vehicle arrival conditions, and ambient conditions pulled from local weather stations, were used as inputs into the model. The information from the experiments and stations was used to construct the process model.

\subsubsection{Compare Larger Process Model against Experimental Data}

While the energy transfer to the stored cryogenic liquid cannot be measured directly the pressure rise in the station tank can be measured. Comparison of the full process model to actual LNG station pressure rise data is used to determine the accuracy of the overall model. LNG stations have a set pressure release point for the station tank PRV (which varies by tank size, station owner, etc.), therefore, by comparing to the pressure rise rate the model can be evaluated for its effectiveness at finding the onset time

of vents. The overall process model along with experimental data is used to gain insight into the driving forces for emission rates from an LNG station PRV and produce estimates for the emissions.

\subsection{Project Scope}

First, the energy transfer model is developed based on the available information. A full process model is required to predict pressure rise and emissions at a station. Therefore, a necessary part of the project scope is to integrate the energy transfer model into the full process model. Pressure rise causes the eventual release of natural gas from a station vent valve, therefore, the model is compared against the pressure rise history of operational stations. The full process model is compared using experimental data 
collected at WVU and two operational LNG stations. Finally estimates using data and the model for vent emissions from the visited stations are given.

\subsection{Thesis Structure}

The remainder of the thesis is structured in the following way.

- Background \& Literature Review (Section 2)

- Model Development (Section 3)

- Data and Model Comparison (Section 4)

- Conclusions (Section 5)

The background and literature review covers research already performed and related to the problem. Since this thesis focused primarily on the development of the energy transfer model the literature review addressed these aspects. However, basic information for understanding the thermodynamics and larger picture was provided. Section 3 briefly outlines the main points of the thermodynamic models but covers the development of the energy transfer portion of the model in detail. Section 4 presents data from two operational LNG stations and compares the full station model. Two full station models were compared, both of which used the same heat transfer model developed in this thesis but utilized two different approaches to the thermodynamics. Also included at the end of Section 4 is a sensitivity analysis. A discussion of the overall study, information resulting from this study, and final concluding remarks are presented in the final section. 


\section{Background \& Literature Review}

\subsection{Liquefied Natural Gas}

Liquefied natural gas (LNG) is the same chemical mixture used to heat homes but is cooled until the gaseous phase has condensed into a liquid at approximately 111.7 K [5]. LNG is not pure methane but may be approximated as such because of its high methane concentration. Typical LNG compositions and sources are shown in Table 2-1 [6].

Table 2-1: Typical LNG Chemical Composition [6]

\begin{tabular}{|l|l|l|l|l|l|}
\hline Source & Methane & Ethane & Propane & Butane & Nitrogen \\
\hline Alaska & 99.72 & 0.06 & 0.0005 & .0005 & 0.20 \\
\hline Algeria & 86.98 & 9.35 & 2.33 & 0.63 & 0.71 \\
\hline Baltimore Gas \& Electric & 93.32 & 4.65 & 0.84 & 0.18 & 1.01 \\
\hline New York City & 98.00 & 1.40 & 0.40 & 0.10 & 0.10 \\
\hline San Diego Gas \& Electric & 92.00 & 6.00 & 1.00 & - & 1.00 \\
\hline
\end{tabular}

Nontoxic, non-corrosive, colorless, and odorless LNG is a relatively safe fuel. However, spilled LNG will vaporize and displace oxygen in the air making large spills an asphyxiation hazard. The flammability limits for vaporized methane, LNG's main component, are an LFL (lower flammability limit) of 5\% and an UPL (upper flammability limit) of 16\% [7]. The advantage of LNG over compressed natural gas is its density, therefore its portability. By liquefying natural gas the density can be increased by up to 600 times; this allows it to be transported across the seas or used in transportation vehicles. Property values for saturated methane are shown in Table 2-2 at $101.3 \mathrm{kPa}$ [5].

Table 2-2: Properties of Saturated Methane at $101.3 \mathrm{kPa}$. [5]

\begin{tabular}{|l|l|l|l|}
\hline Property & Liquid Value & Vapor Value & Unit \\
\hline Boiling Point & 111.7 & 111.7 & $\mathrm{~K}$ \\
\hline Density $(\rho)$ & 414.1 & 1.792 & $\mathrm{~kg} / \mathrm{m}^{3}$ \\
\hline Latent Heat $\left(\mathrm{H}_{\mathrm{v}}\right)$ & 512 & - & $\mathrm{kJ} / \mathrm{kg}$ \\
\hline Specific Heat $(\mathrm{Cp})$ & 3.461 & 2.172 & $\mathrm{~kJ} /(\mathrm{kg}-\mathrm{K})$ \\
\hline Viscosity $(v)$ & 0.118 & .00447 & $\mathrm{mPa}-\mathrm{s}$ \\
\hline Thermal Conductivity $(\mathrm{k})$ & 0.193 & .01188 & $\mathrm{~W} /(\mathrm{m}-\mathrm{K})$ \\
\hline
\end{tabular}




\subsection{LNG Vehicle Stations}

\subsubsection{Overview}

Many configurations of LNG vehicle fueling stations exist but the basic components of a station are shown in Figure 2-1 [8]. The first component group, the offload piping, included the dedicated tanker truck offloading pump, the vapor return to the tanker truck, and associated piping. The offload piping was used to refill the station tank after it was low or depleted of LNG. Typically, station refilling only occurred when the station dispensed enough fuel to vehicles to accept a full tanker load of LNG. Therefore, the duration the station tank remained below venting pressures was greatly dependent on the number of customers it served. The station tank was a cryogenic dewar usually operating at pressures below $1725 \mathrm{kPa}$ (250 psia). When pressures reached a high enough value ( 1000 kPa to $1300 \mathrm{kPa}$ ) the auto-vent pressure regulator or PRV was activated and methane vapor was released until the pressure lowered sufficiently or until the next offload event occurred.

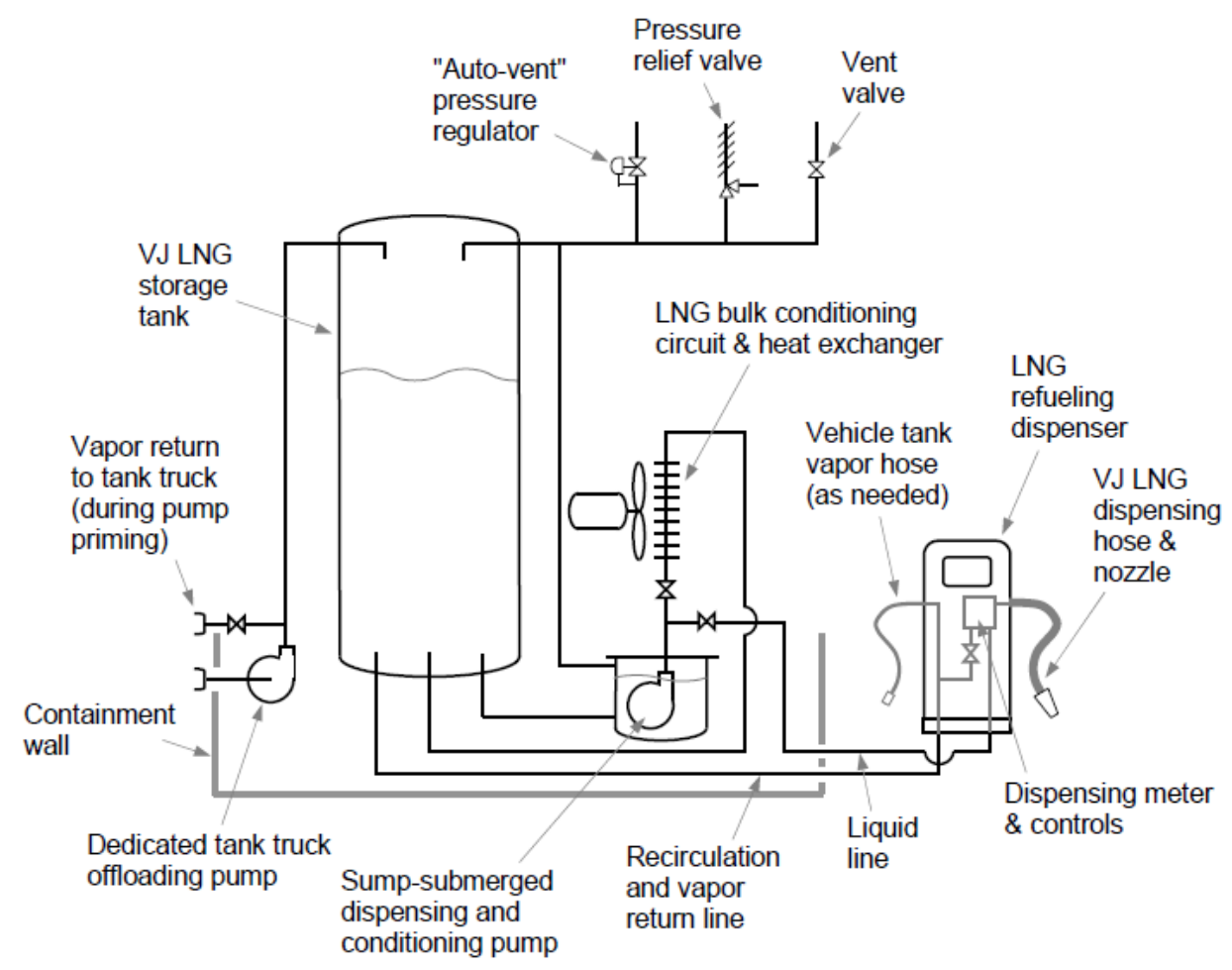

Figure 2-1: Simplified LNG Station Layout [8]

The dispense piping of the station included the dispensing pump, recirculation lines, and vapor return line. The dispense pump pressurized the LNG inside the tank and transferred it to the dispenser for fueling of LNG vehicles through the recirculation lines. The vapor return line was used to accept vapor vented back to the station from vehicle tanks. Vehicles sometimes arrived at the station at a pressure too high for efficient 
fueling and released pressure to the station tank through the vapor return line. The recirculation lines consisted of the piping connecting the dispenser to the station tank. The recirculation lines' name referred to the process utilized to keep the lines cold, which was to circulate cold LNG in the tank through the piping. Unlike the recirculation lines the refueling dispenser was not constantly cooled. Each time a vehicle arrived at the station, the dispenser first went through a cool down process before fueling. The LNG bulk condition circuit and heat exchanger was used for multiple purposes. Many stations conditioned or warmed the LNG from a tanker offload especially if vehicles arriving at the station required warmer fuel to operate. Warming the LNG, however, reduces the time the fuel can be stored before venting begins to occur. Therefore, usually the LNG was kept as cold as possible. Another method was to use the heat exchanger to actively warm the fuel upon dispensing. In addition, some stations utilized liquid nitrogen (LN) and a heat exchanger to insure no venting occurred by using the $\mathrm{LN}$ as a refrigerant.

\subsubsection{LNG Station Vehicle Fleet}

LNG station fleets were characterized by what type of technology the vehicle used for engine power and LNG storage. The two major types were HPDI (high pressure diesel injection) and Stoichiometric. HPDI technology used a pump installed inside the cryogenic tank to provide high pressure LNG to the engine. A small amount of diesel was injected into the engine, to provide a source of ignition, along with the pressurized methane. Stoichiometric engines required the LNG to be vaporized and were spark ignited engines. Stoichiometric engines did not have an internal fluid pump inside the vehicle tank to supply LNG from the tank to the engine. Stoichiometric vehicles relied on the tank pressure along with a heat exchanger, and an economizer, to supply fuel to the engine. The economizer behaved as a regulator allowing some vapor to be used from the tank when the pressure was high enough, instead of the liquid LNG passing through the heat exchanger; this helped regulate the pressure. Otherwise the heat exchanger vaporized LNG for the engine. Stoichiometric vehicles required a warmer LNG product than HPDI vehicles. Dependent on fleet compositions the temperature of LNG at a fueling station may be maintained at different states. The temperatures were referred to in the industry as cold (approximately $125 \mathrm{~K}$ to $135 \mathrm{~K}$ ) or saturated (warmer, approximately $135 \mathrm{~K}$ to $150 \mathrm{~K}$ ) fuel. Saturated fuel was a misnomer from an engineering stand point because it did not mean thermodynamically saturated. 


\subsection{LNG Storage Tanks}

\subsubsection{Overview}

LNG storage tanks varied but were mainly limited to large storage for production plants, LNG transportation (marine vessels, train, and truck), vehicle fueling station storage, and fuel tanks for vehicles. Figure 2-2 through Figure 2-5 show these various storage methods.

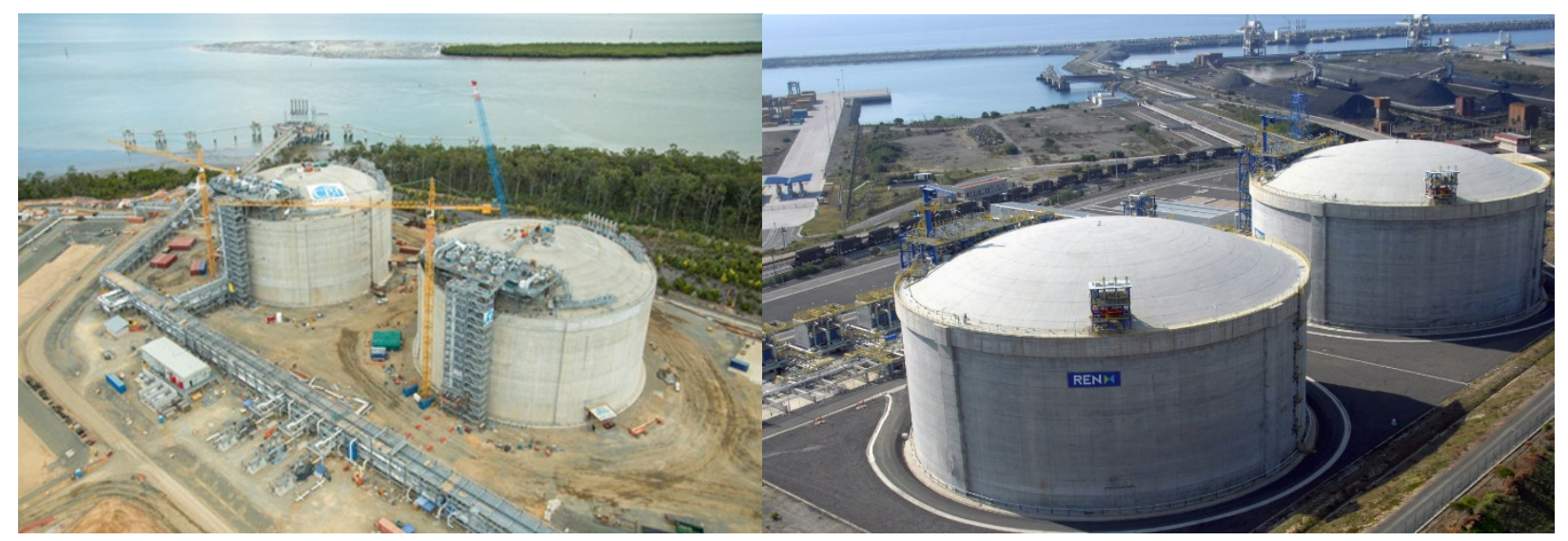

Figure 2-2: Flat Bottomed Large Cryogenic LNG Storage Tanks [9], [10]
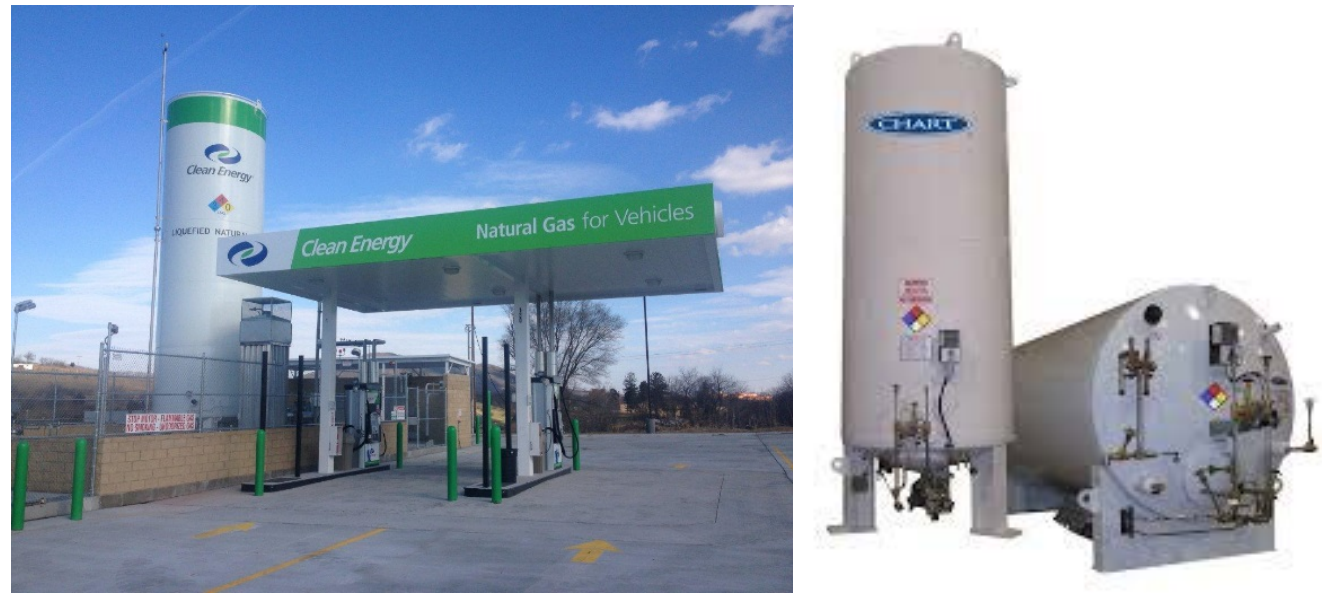

Figure 2-3: Horizontal and Vertical LNG Storage Tanks for Vehicle Fueling Facilities [11], [12] 

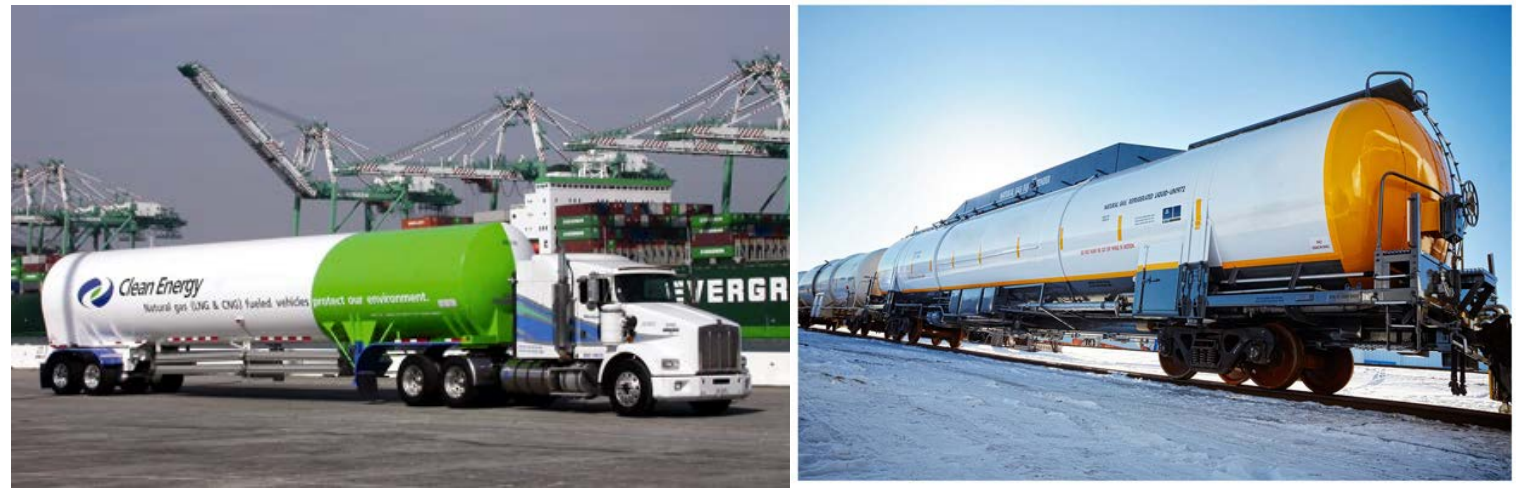

Figure 2-4: LNG Transport Tankers [8], [12]

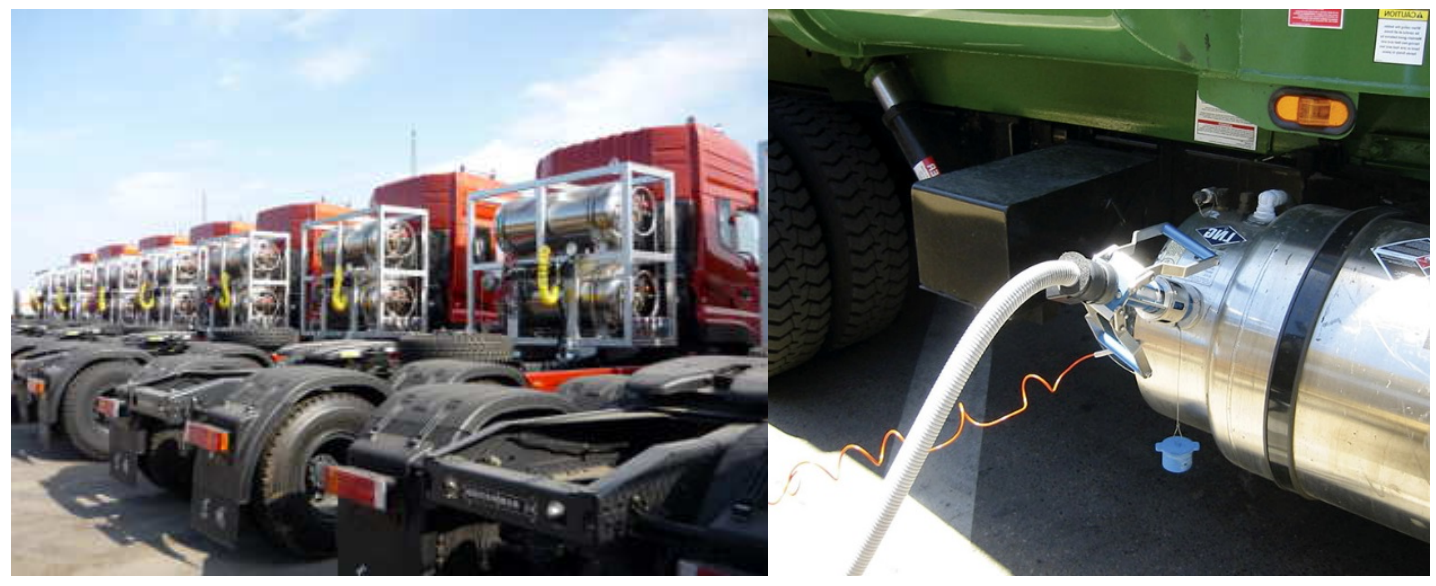

Figure 2-5: LNG Vehicle Fuel Tanks [12]

As a cryogenic fuel, LNG required special construction of its storage tanks. The storage tanks were designed to sufficiently limit the heat transfer into the fuel from the ambient air. Temperatures of fuel stored inside the tank ranged based on the pressure of the tank, but with a possible low of $-160^{\circ} \mathrm{C}\left(-256^{\circ} \mathrm{F}\right)$. With an ambient air temperature of $20^{\circ} \mathrm{C}\left(68^{\circ} \mathrm{F}\right)$, this produced a temperature difference of around $180^{\circ} \mathrm{C}$ $\left(356^{\circ} \mathrm{F}\right)$. Heat input into the tank resulted in vaporization (boil off) of LNG. To maintain safe operating pressures all tanks were equipped with a pressure relief valve (PRV). Any fuel vented to the atmosphere was referred to as BOG (boil off gas).

\subsubsection{Dewar Design Features}

LNG storage tanks and most high performance cryogenic storage tanks were similar in design. The construction was based on the designs of Sir James Dewar. A dewar is a double walled container with the space in-between, the annulus, evacuated to a vacuum. Typical dewar design features for cryogenic vessels are shown in Figure 2-6. 


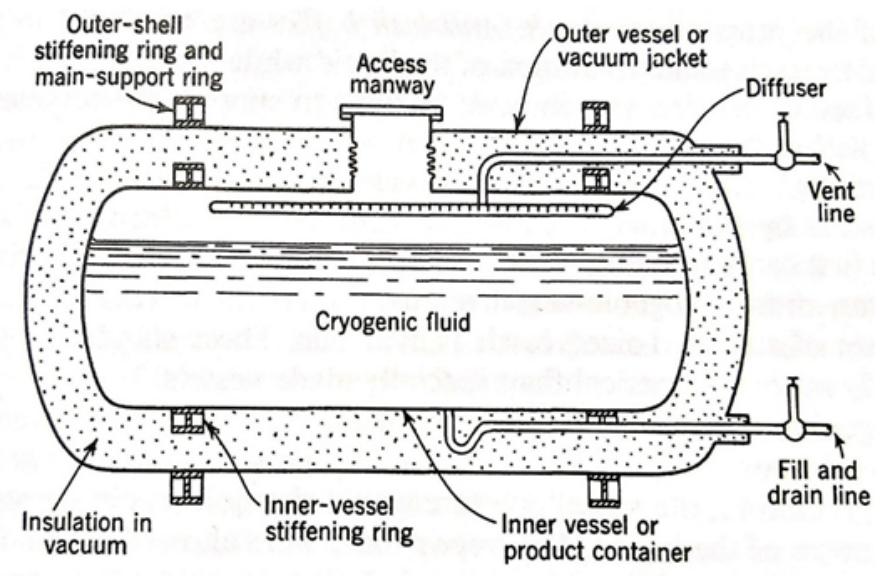

Figure 2-6: Dewar Design Features [13]

The vessels were constructed of materials cryogenically suitable such as stainless steel, aluminum, monel, and copper. An effective cryogenic material was one which did not become brittle at the cryogenic fluid temperatures (123 K and below); in general these were materials with face centered cubic crystal structures [13]. The inner vessel was held up by supports of different configurations connected to the outer shell wall. The configuration of supports depended upon the application. A LNG station tank required different supports than a vehicle tank which may be subject to collision.

Figure 2-7 shows some typical configurations used in the industry. Figure 2-8 shows additional details of strut construction [13].

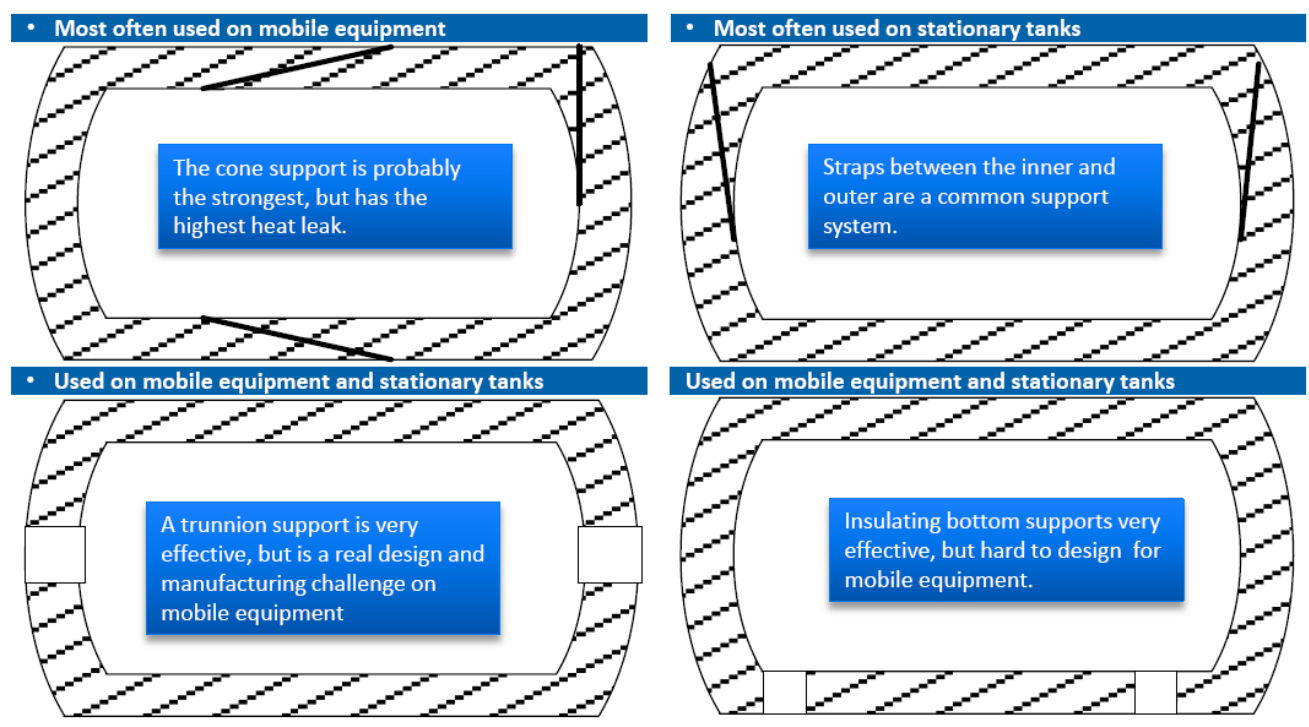

Figure 2-7: Tank Support Approaches [14] 


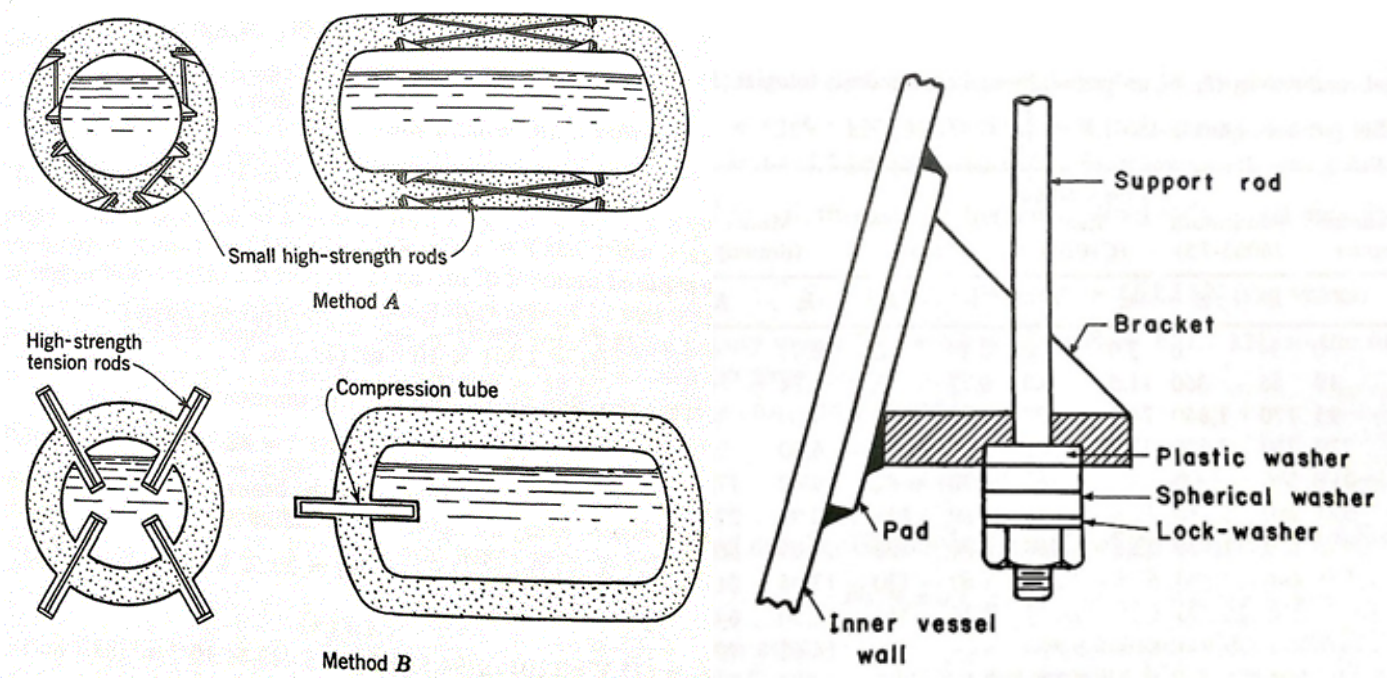

Figure 2-8: Dewar Strut Supports [13]

Heat transfer into the stored cryogenic fluid was mitigated by advanced insulation. Never the less conduction occurred through the structural supports bridging the gap between the inner and outer layer of the dewar. Convection still occurred but to a limited extent because of the high vacuum pulled in the annulus; less than 1 millionth of an atmosphere. Radiation heat transfer from the outer shell to the inner constituted a major portion of heat input and was typically what advanced insulations attempted to prevent. Vehicle tanks and station tanks were constructed similarly in that both had the basic features of cryogenic dewars. A more in-depth analysis of vehicle tanks was presented by O’Brian et al. [15].

\subsubsection{Materials and Insulation}

Conduction occurred largely through the tank support material (the struts) and it was not possible to eliminate, only reduced by specialty strut materials and/or minimizing the amount of strut material used. Insulation used effectively in the annulus greatly increased the performance of a cryogenic tank. There were many methods and materials used for insulation. The various insulation materials commonly used included fiberglass, foam, Perlite, Aerogel and Multilayer Insulation (MLI) also called super insulation [7]. Fiberglass, perlite, and aerogel insulations were usually formed into beads/powder and packed into the annulus of the dewar. MLI consisted of layers of a reflective material (such as aluminum foil) and spacer material (such as fiberglass paper). At atmospheric pressure all were relatively poor insulators but as vacuum increased the bead insulations and MLI became rapidly more effective. MLI insulation was commonly used to achieve high performance storage for LNG tanks. Table 2-3 gives some density and thermal conductivity values for powder and fibrous insulation materials. 
Table 2-3: Properties of Evacuated Powders and Fibrous Insulation Materials. [13]

\begin{tabular}{|l|l|l|}
\hline Insulation & Density $\left(\mathrm{kg} / \mathrm{m}^{3)}\right.$ & Thermal Conductivity $(\mathrm{mW} / \mathrm{m}-\mathrm{K})$ \\
\hline Fine perlite & 180 & 0.95 \\
\hline Coarse perlite & 64 & 1.90 \\
\hline Silica aerogel & 80 & 1.60 \\
\hline Calcium silicate & 210 & 0.59 \\
\hline Lampblack & 200 & 1.20 \\
\hline Fiberglass & 50 & 1.70 \\
\hline
\end{tabular}

The thermal conductivity of MLI was around 10 times better at low vacuum than beads (powdered) and fibrous insulations. The thermal conductivity of MLI was dependent on the vacuum level, boundary temperatures, and number of layers used [16],[17].

Table 2-4 gives some values for the mean thermal conductivity of 40 layers of MLI at different boundary temperatures. There was a rapid loss of thermal resistance in the tank as the vacuum in the annular space approached atmospheric pressure; therefore, proper tank maintenance and periodic reconditioning was critical. When the vacuum was lost the insulation reverts to that of a thin non-jacketed tank.

Table 2-4: Mean Thermal Conductivity of MLI in Vacuum at Various Boundry Temperatures [16]

\begin{tabular}{|l|l|l|}
\hline $\mathrm{T}_{\mathrm{C}}(\mathrm{K})$ & $\mathrm{T}_{\mathrm{H}}(\mathrm{K})$ & $\mathrm{K}_{\text {mean }}(\mathrm{mW} / \mathrm{m}-\mathrm{K})$ \\
\hline 77 & 400 & 0.117 \\
\hline 77 & 350 & 0.081 \\
\hline 77 & 300 & 0.055 \\
\hline 77 & 250 & 0.038 \\
\hline 77 & 200 & 0.027 \\
\hline 77 & 150 & 0.021 \\
\hline
\end{tabular}

Stainless Steel 304 was a common building material for cryogenic applications. It was extensively used in LNG storage tanks and was used in many cases for strut supports, outer layer, and inner containers of tanks. Figure 2-9 shows the variation in thermal conductivity with temperature of stainless steel [18]. 


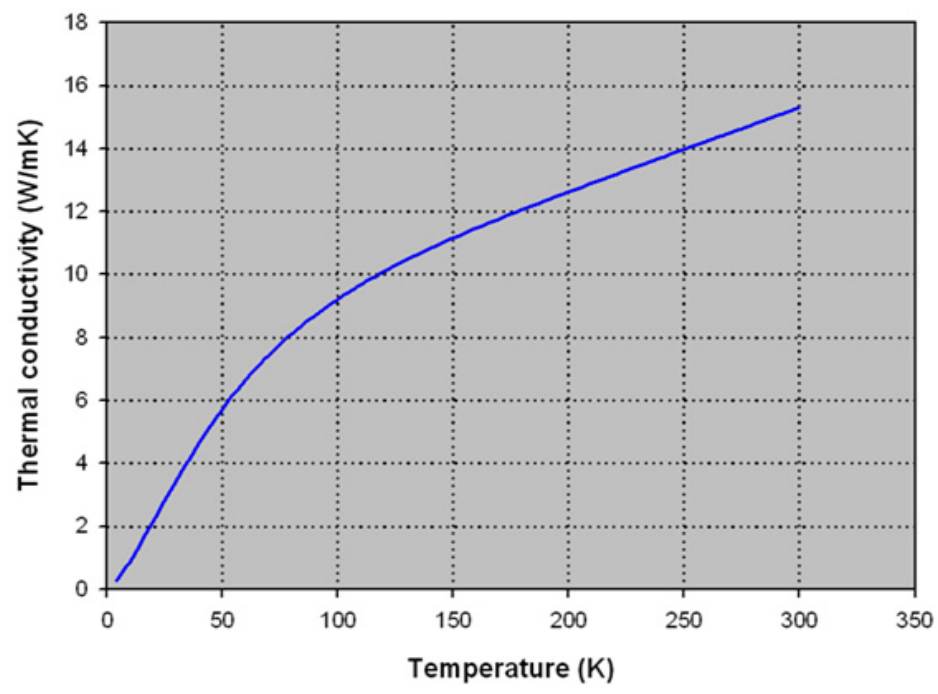

Figure 2-9: Thermal Conductivity of Stainless Steel 304 from 4 K to 300K [18]

Additional heat transfer occurred from thermal conduction through process piping which passed between the inner and outer vessel liners. Special care was standardly taken in the design process to limit heat flux through these surfaces. For a properly designed system conduction occurred only along the pipe wall and heat transfer was greatly reduced.

\subsection{Cryogenic Transfer Lines}

Transferring cryogenic fluid over a few feet was achieved well through thin walled bare pipe but in a fuel station setting where many meters of piping was present more efficient means existed. Foam insulation was added to the pipe to increase the thermal resistance. For the lowest heat transfer rates vacuum jacket piping (VJP) and vacuum jacketed multilayer insulation piping (MLIP) was installed. These insulation strategies proved greatly advantageous to a LNG station because the LNG in the transfer lines was preserved and limited the heat load on the station.

\subsubsection{Frost Formation on Bare Pipe}

Bare pipe grows a frost layer on its surface when transferring cryogenic fluid. The frost layer behaves as a natural insulation reducing the normally large heat transfer rate. Frost layer formation is dependent on many factors including local humidity conditions, the temperature of the surface of the pipe, external convection, etc. [19]. In addition, the thermal conductivity of the frost changes the longer it is present on a bare pipe. As Sahin described, thermal conductivity of a frost layer with a density of $200 \mathrm{~kg} / \mathrm{m}^{3}$ ranged from 0.13 to $0.2 \mathrm{~W} / \mathrm{m}-\mathrm{K}$. A variation of $20 \%$ around the average thermal conductivity for one frost density. Frost densities vary from 0 (no frost) to $934 \mathrm{~kg} / \mathrm{m}^{3}$ (solid ice). 


\subsubsection{Foam Insulated Pipe}

Foam insulations were economical in many respects for cryogenic applications and were used commonly for pipe bends in amongst vacuum jacketed piping at LNG stations. There were multiple types of foam insulation including polyurethane (PUR), glass fiber foam, and Cetrafoam [20]. Polyurethane was one of the most commonly used foam insulations. It had a thermal conductivity ranging between 21.4 to 37 $\mathrm{mW} /(\mathrm{m}-\mathrm{K})$ depending on specific additives [21]. A draw back to PUR foam insulation was its tendency to deteriorate more quickly than other insulations over time. In addition, PUR was known to absorb moisture in surrounding air which increased the thermal conductivity of the foam and further hastened the decay rate [20], [22].

\subsubsection{Vacuum Jacket Piping \& MLI Piping}

For the long lengths and high time duration of cryogenic fluid transfer vacuum jacketed piping (VJP) and multilayer insulated piping (MLIP) were the optimal piping choice. VJP and MLIP piping were similar in design and construction. Both employed a vacuum to reduce conduction and convection heat transfer. MLIP gained the benefits of reducing the radiative heat transfer mode and was used extensively in LNG situations. Like in cryogenic tanks some supportive material was necessary to space the central pipe away from the outer shell. Figure 2-10 shows some spacer configurations [13].

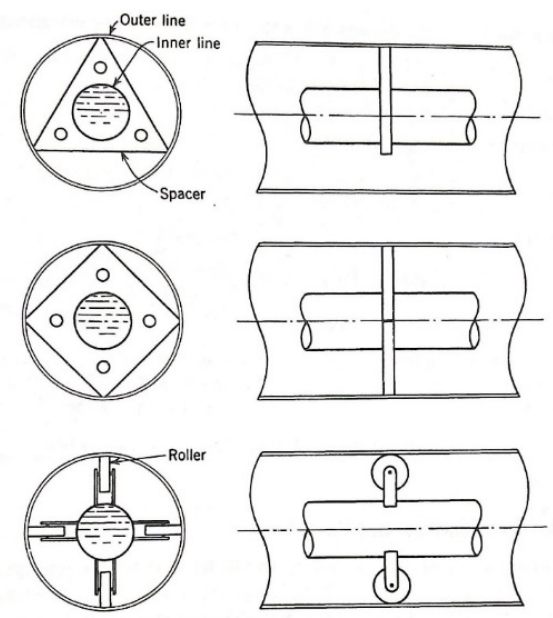

Figure 2-10: VJP \& MLI Spacer Configurations [13]

Not all MLIP was created equal. Performance of MLIP varied among manufactures and until recent advancement performed about 10 times worse than MLI found in cryogenic tanks [23]. Dye et al. in 2014 detailed methods used in the construction of MLIP to increase performance. Older MLIP methods using 5 layers of MLI at boundary conditions of 77-295 K had a heat flux of around $26.6 \mathrm{~W} / \mathrm{m}^{2}$. The new method of applying MLI to the piping was able to decrease the heat flux to $2.2 \mathrm{~W} / \mathrm{m}^{2}$. 


\subsection{Cryogenic Fluid Stratification}

Fluid stratification in cryogenic tanks is an important phenomena which effects not only the thermodynamic state of the tank but the heat transfer entering the fluid. Stratification is the presence of a thermal or density gradient in the fluid. Most LNG station storage tanks did not have an appreciable density gradient but large tanks (15-18 million gallon) existed with density gradients. More information about the effects of density gradients on the large tanks was presented in [24]. Heat transfer through the vessel insulation causes fluid to warm, giving rise to a convection current and thermal stratification much to the effect seen in Figure 2-11 [25]. Stratification has been observed to be stable in many cryogenic tanks, and has been observed to produce upwards of a $15 \mathrm{~K}$ temperature gradient even in small (2 liter) tanks [26], [27]. The intensity of stratification depends on many factors and is still an area of research. Stratification is known to affect the pressurization rate of cryogenic tanks. Since the liquid-vapor interface is at a higher temperature than a well-mixed saturated state the pressure rise rate increases. While pressure rise rate increases, the heat transfer rate decreases due to the increased internal temperature (lower driving temperature gradient) and slowing convection [28].

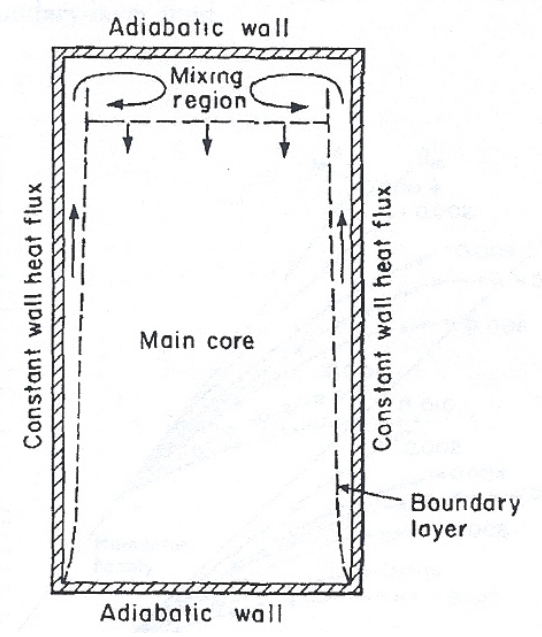

Figure 2-11: Convection in an Enclosed Tank [25]

Figure 2-12 shows an example of thermal stratification measured by experiment from reference [27]. The working fluid was LN and a small 2 liter tank was used. The vertical axis in the figure is the depth of the fluid in the tank and each line represents a different time. Larger tanks may expect greater stratification effects. 


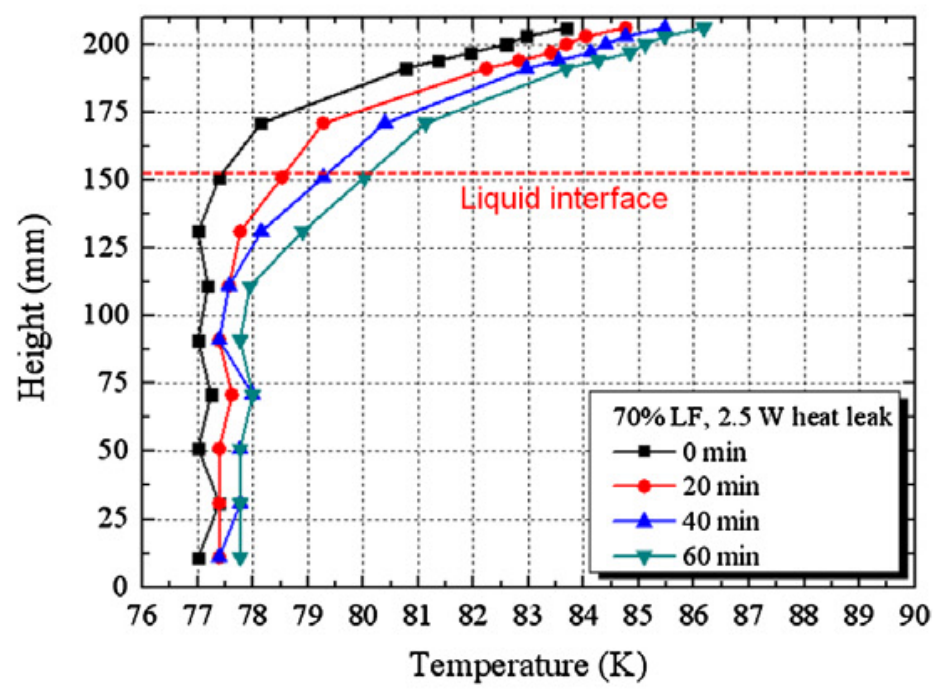

Figure 2-12: Example of Stratification in Liquid Nitrogen [27]

Roll over is a phenomenon associated with stratification which occurs when a large portion of the fluid within a tank quickly vaporizes. The large mass of suddenly vaporized liquid causes a dangerously fast increase in pressure within the tank. To date no accidents from rollover resulted in casualties but instances of the destruction of a tank have been observed. Bates et al. [29] presented an in depth investigation into cryogenic stratification and the roll over phenomenon. In addition, Kiran Deshpande et al. investigated predicting time to roll over in reference [30].

\subsection{Cryogenic Tank Models}

Many research papers and reports exist concerning the modeling of pressure rise in cryogenic tanks. The approaches used to calculate pressure rise vary by computational methods and thermodynamic approach. The following papers give excellent information from research related to pressure rise models and the topic at hand.

References [27], [31], [32], [33], [34], [35], and [36] dealt in detail with the issue of stratification in cryogenic tanks. Seo et al. produced a paper in 2009 which shows experimental data of stratification in a cryogenic vessel using liquid nitrogen [27]. Descriptions of two thermodynamic models were then given and compared to the experimental data. A simplified thermodynamic approach to stratification was developed by Riemer for LNG and compared to experimental data in [31]. A case study of three different heating regimes for a tank were explored with one thermodynamic approach [32]. Stratification was modeled in all heating modes and a jet used for mixing the fluids was also investigated. Herman et al. used a finite difference approach to calculate stratification and pressure rise [33]. Reference [34] was produced 
by NASA and was an excellent source of information on stratification. Natural convection theory was used to calculate the stratification build up over time and a reference code in FORTRAN was provided. A work investigating thermal stratification and de-stratification with FLUENT software was presented in 2012 [35]. An experiment investigating stratification using R22 was conducted by Shi et al. [36].

CFD models were covered in depth in references [37] and [38]. Barsi et al. [37] described a two phase CFD model and comparison of the model to data collected by NASA. Information about the computational cost for CFD was discussed. Simulations were, “approximately 4 days of CPU time on 6 AMD 64 bit Opteron processors to simulate the 20 hour experiment” [37]. From this insight CFD was avoided for the purposes of this project.

The dynamics of the liquid-vapor interface are important to pressure rise in a cryogenic tank. Modeling work for bulk evaporation and condensation at the liquid-vapor interface was presented by Anghaie et al. [39]. Two iterative methods were developed in the paper and then numerical experiments were conducted to determine computational efficiency and accuracy.

Relatively few papers used a heat transfer model coupled to a thermodynamic model for the calculation of pressure rise. A helpful work for approaching the heat transfer in a cryogenic tank and LNG station was found in Chen et al. [40]. A formulation for calculating the heat transfer through a cryogenic tank wall was presented using a 1-D resistance network approach. Other sources of heat loading were considered such as heat leak through the dispenser. Each of these heat sources was then tied to a simple thermodynamic model and the pressure rise rate was calculated. In addition, the paper gave numbers estimating BOG from a station for specific heat loads. Another important paper by Li et al. [28] discussed research on how pressure rise rate slows as fill level drops in vertical tanks. The paper posited that the heat transfer rate was lower at lower fill levels and gives a correction factor for heat leak calculation.

\subsubsection{Additional Model Research Information}

Additional related literature which was not used in the model development but gives deeper insight into the pressurization and BOG problem follows. Information on the heat transfer into large bulk storage tanks, located at production facilities, was found in Chung et al. [41]. A heat transfer model was proposed in the paper and then evaluated by collected data. Another case study of boil off gas and heat leak from large LNG storage tanks was performed by Adom et al. in 2010 [42]. The heat leak values for multiple size

tanks were provided along with the emission behavior of the storage tanks. Efficiency of LNG tank operation for the reduction of BOG in large storage tanks was found in Park et al. [43]. The paper described best operational practices for a large storage tank operator.

Hofmann [44] in 2006 created a process for estimating the pressurization period of a cryogenic tank quickly, using charts. The charts were created by assuming an isochoric change of state for the 
thermodynamics. If the heat leak was known then the pressurization period was calculated based on the charts. Gorla et al. [45] extended the charts to include LNG, LPG, and R134a.

Ludwig et al. [46] discussed pressure rise and drop in cryogenic tanks under oscillations much like a vehicle tank would be subject too. In the study six different shaking conditions were investigated on a

0.148 meter diameter tank. Pressures started at approximately $250 \mathrm{kPa}$ and pressure drops caused by the oscillations were recorded to as low as $150 \mathrm{kPa}$. 


\section{Model Development}

\subsection{LNG Station Energy Transfer Model Development Introduction}

The energy transfer model developed in this thesis is a submodule of the full station model used to calculate the pressure rise and emissions from an LNG station. The full station model includes two thermodynamic approaches for calculating pressure rise, a venting module which calculates emissions from the station PRV and vapor return of vehicle tanks, both developed by Sandoval, the energy transfer model developed here, and other tertiary modules used to handle inputs and data. MATLAB/Simulink was used to develop the energy transfer model and the full station model. Simulink utilized imbedded function blocks, and premade operator blocks to simulate the station. MATLAB scripts were used by the model to input initial conditions and dimensions into the model. Once these initial conditions were given to Simulink the model was then run by marching through time at a pre-defined rate usually in 10 minute intervals. The role of the energy transfer model was to calculate the heat and energy input to the stored LNG driving the pressurization of the station tank.

Five major component sets were grouped and then analyzed for the calculation of the heat and energy load on a LNG station. The components evaluated were the station storage tank, offload piping, dispense piping, vapor return line, and dispenser. The station storage tank was considered in the vertical orientation. Connected to the tank was the offload piping and the dispense piping. The offload piping was used for the refueling of the station bulk tank. Dispense piping delivered fuel from the station tank to the fuel dispenser. At the fuel dispenser was a vapor return line from the dispenser to the station bulk tank. The vapor return line allowed vehicles arriving at the station to vent down to a pressure where the station could refill the vehicle tank efficiently. The modeled components are shown in Figure 3-1.

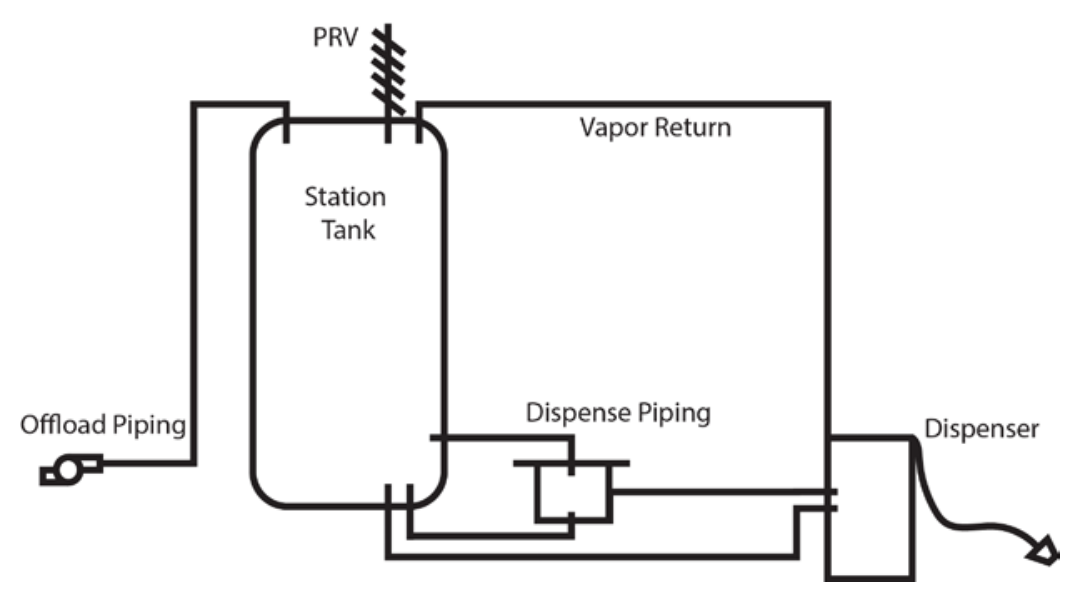

Figure 3-1: Station Components Modeled for Energy Transfer to Storage Tank LNG 
Multiple LNG stations were visited during the study. At two stations a long term audit of approximately three weeks occurred during which data were collected. One station was located in Nevada and referred to as audit 1 or the audit 1 station. The audit 2 station was located in Utah. Data for pressure and fill level were gathered along with vehicle fueling information. The analysis for a station was based on experience gained during the audits and with cooperation from station owners.

\subsection{Station Tank Heat Transfer}

Derived in this section are the necessary equations for heat transfer through a vertical storage tank. Equations for a horizontal storage tank are found in Appendix A. The objective of the energy transfer model was to provide the total energy transfer rate input in Watts to the stored LNG inside the cryogenic tank. The thermodynamic approach for calculating the pressure rise in the tank changed composition of some of the algorithms used for the heat transfer but the basic approach was not modified.

Figure 3-2 shows a schematic of a LNG tank with associated heat transfer variables and characteristics. The tank was of height, $L$ and was filled with LNG assumed to be pure $\mathrm{CH}_{4}$ to a fill level $L_{f}$. The radius to the inner surface of the inner shell of the tank was $r_{1}$. The radius to the outer surface of the tank was $\mathrm{r}_{4}$. The annulus between the inner and outer shells contained multilayer insulation (MLI) under high vacuum with a thermal conductivity $k_{\text {MLI. }}$ The inner shell of the tank was supported by struts of thermal conductivity $\mathrm{k}_{\text {strut. }}$ Various plumbing connections penetrated through the insulation into either the liquid or vapor spaces. These plumbing connections were considered well insulated. The LNG was stored at a temperature $T_{f}$ and produced a convective heat transfer coefficient $h_{\mathrm{i}, f}$ at the inner surface of the tank. The $\mathrm{CH}_{4}$ vapor existed at temperature $T_{\mathrm{g}}$ and created a convective heat transfer coefficient $h_{\mathrm{i}, \mathrm{g}}$ at the inner surface of the tank. The outer surface of the tank was subject to heat transfer via convection from the atmosphere with velocity $U_{\infty}$ and temperature $T_{\infty}$; this resulted in a heat transfer coefficient at the outer surface of the tank of $h_{0}$. Portions of the tank surface were also exposed to direct solar radiation $G_{\text {solar }}$ The outer surface of the tank was characterized by solar absorptivity $\alpha_{\mathrm{s}}$ and emissivity $\varepsilon$. 


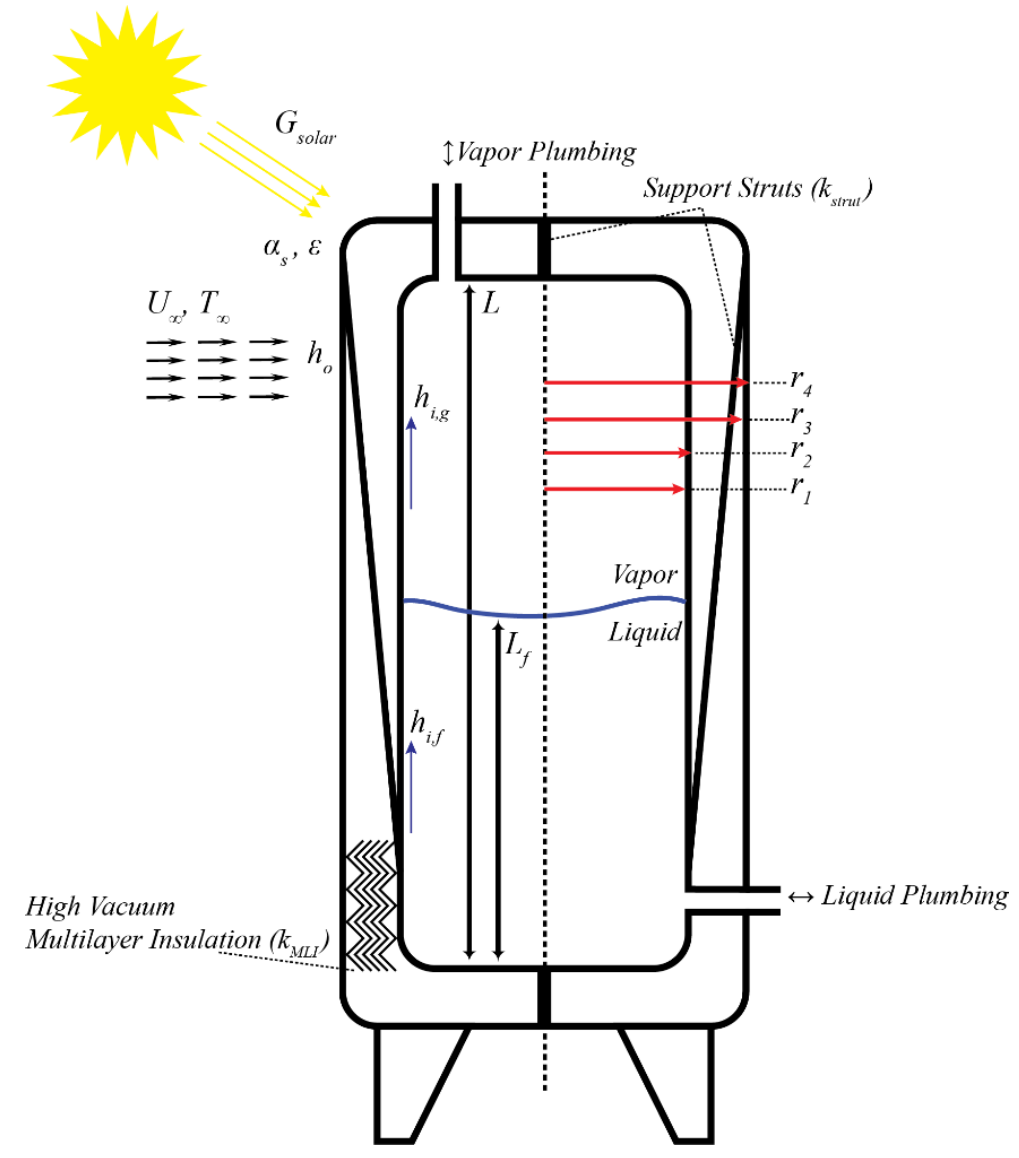

Figure 3-2: Station Tank Diagram

The exact design of the internal supports and insulation were not known. Since internal dimensions were not known exactly an approach similar to [40] was followed. The tank surface was subdivided, each section analyzed and the heat input rates summed to produce an overall heat transfer rate.

LNG storage tanks are constructed as a dewar with an evacuated annulus used in conjunction with an evacuated insulation. In this construction a relatively small amount of strut material supports the inner tank. The strut material has much greater thermal conductivity as compared to multi-layer insulation's equivalent conductivity. Therefore, the construction of the tank was conceptualized as a 1-D resistance network shown in Figure 3-3. Externally, convection with air, direct radiation (sunlight) on a percentage of the tank's surfaces, and diffuse solar radiation created heat transfer into the LNG tank. Convection with the air occurred through natural convection (no wind blowing) and/or forced convection (wind). Heat gained from the outside sources was transmitted by conduction to the inner tank surface and LNG. The primary path for conduction was through the strut material. Heat transfer through the insulation was modeled as conduction with the use of an equivalent thermal conductivity. The thermal conductivity of MLI for purposes of this study was considered constant at $0.05(\mathrm{~mW} / \mathrm{m}-\mathrm{K})$. The ratio relating support junction area and insulation area was a defining characteristic of the tank called the area ratio. The ratio was defined as 
$\gamma=A_{\text {strut }} / A_{\mathrm{S}}$, where $A_{\text {strut }}$ was the cross sectional surface area of the supporting strut material and $A_{\mathrm{s}}$ was the surface area of the outside of the tank. Lastly, the inner surface transferred heat to the LNG through natural convection.

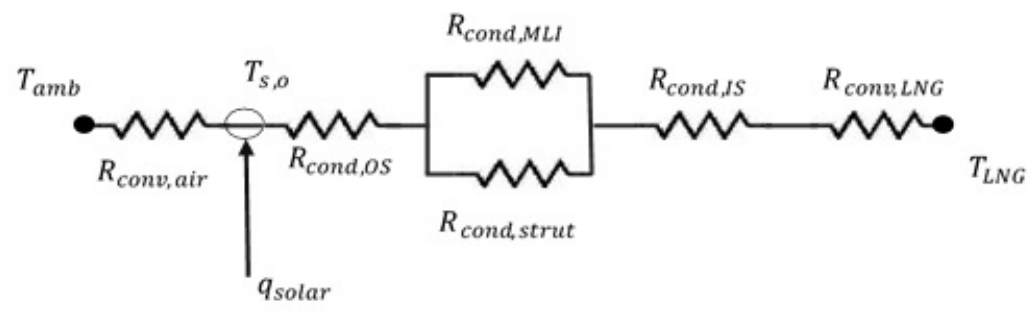

Figure 3-3: Station Tank Resistance Network

The resistance network was applied to all portions of the tank by taking into account the different geometries and environmental conditions. Stratification was handled similarly by discretizing the tank walls. Starting with the tank wall itself, the resistance of the tank wall was expressed as found in Equation 1.

$$
R_{\text {Tank }}=R_{\text {cond,os }}+\frac{R_{\text {cond, } \mathrm{MLI}} * R_{\text {cond,strut }}}{R_{\text {cond,MLI }}+R_{\text {cond,strut }}}+R_{\text {cond,IS }}
$$

In Equation 1, $\mathrm{R}_{\text {cond,os }}$ was the resistance of the outer shell of the dewar, $\mathrm{R}_{\text {cond,strut }}$ was the resistance of the strut material, $\mathrm{R}_{\text {cond,MLI }}$ was the resistance of the MLI, and $\mathrm{R}_{\text {cond,IS }}$ was the resistance of the inner tank wall. The tank was divided into regions and the appropriate correlations and forms of equations were used for each. The cylinder portion of the tank was considered one region and the end caps were considered the other regions. Depending on the tank orientation (vertical or horizontal) different correlations for convection heat transfer were used but the conduction equations remained the same.

\subsubsection{Cylinder Region}

The following equations and correlations for the mid-section of the cryogenic tank were developed for a vertical tank with the resistance network found in Figure 3-3. First, the equations needed for the resistance of the tank wall were considered as expressed in Equation 1. The outer shell resistance was expressed in cylindrical coordinates as found in Equation 2.

$$
R_{\text {cond }, O S}=\frac{\operatorname{Ln}\left(r_{2} / r_{1}\right)}{2 \pi k_{m, 1} L}
$$

Where $\mathrm{L}$ was the height of the region. The parameter $\mathrm{k}_{\mathrm{m}}$ was the mean thermal conductivity and $\mathrm{r}_{2}$ and $\mathrm{r}_{1}$ were the radii shown in Figure 3-2. 
For calculation of the heat transfer through the annulus the area ratio $(\gamma)$ was incorporated. It was used in Equations 3 and 4. The area ratio was taken to be the cross-sectional area of cumulative support material divided by external surface area of the entire tank. Calculation procedures and estimates for the area ratio are found in Appendix A.

$$
\begin{gathered}
R_{\text {cond,strut }}=\frac{\operatorname{Ln}\left(r_{3} / r_{2}\right)}{2 \pi \gamma k_{\text {strut }} L} \\
R_{\text {cond,MLI }}=\frac{\operatorname{Ln}\left(r_{3} / r_{2}\right)}{2 \pi(1-\gamma) k_{M L I} L}
\end{gathered}
$$

Convection on the external surface of the tank was handled by using the appropriate convection correlations for the geometry. Equation 5 was used to calculate the convection resistance on the surface of the vertical tank for natural convection.

$$
R a_{L}=G r_{L} \operatorname{Pr}=\frac{g \beta\left(T_{s}-T_{\infty}\right) L^{3}}{v^{2}} * \operatorname{Pr}
$$

The Rayleigh $\mathrm{Ra}_{\mathrm{L}}$ number was used in the calculation of Nusselt number shown in the correlation Equation 6. The equation is valid for vertical cylinders so long as $\frac{D}{L} \geq \frac{35}{G r_{L}^{1 / 4}}$ [47].

$$
N u_{L}=0.68+\frac{0.670 R a_{L}^{1 / 4}}{\left[1+(0.492 / P r)^{9 / 16}\right]^{4 / 9}} ; R a \leq 10^{9}
$$

Forced convection followed a similar approach to natural convection with Reynolds number used to characterize the flow.

$$
R e_{D}=\frac{V_{\infty} D}{v}
$$

The correlation for forced external flow of over a cylinder was given by Equation 8 . Where the function is valid for all $\operatorname{Re} * \operatorname{Pr} \geq 0.2$ [47].

$$
\overline{N u}_{D}=.3+\frac{0.62 \operatorname{Re}_{D}{ }^{1 / 2} \operatorname{Pr}^{1 / 3}}{\left[1+(0.4 / P r)^{2 / 3}\right]^{1 / 4}}\left[1+\left(\frac{R e_{D}}{282000}\right)^{5 / 8}\right]^{4 / 5}
$$

And the convection coefficient was calculated by using the Nusselt number where $L$ was used for the natural convection case and D (the diameter) for the forced convection case. 


$$
h_{\text {conv }}=\frac{N u k_{\text {air }}}{L}
$$

The resistance was then calculated by Equation 10 .

$$
R_{\text {conv, air }}=\frac{1}{h_{\text {conv }} A}
$$

Natural convection was considered negligible if $\mathrm{Gr} / \mathrm{Re}^{2}$ was much less than unity and vise vera for forced convection. When $\mathrm{Gr} / \mathrm{Re}^{2} \approx 1$ both forced and natural convection have comparable values. Equation 11 was used as a first approximation for finding the combined Nusselt number [47]. Where $\mathrm{Nu}_{\mathrm{F}}$ was the Nusselt number from forced convection, $\mathrm{Nu}_{\mathrm{N}}$ was the Nusselt number from natural convection, and $\mathrm{n}$ depended upon the geometry. For vertical plates and vertical geometries $n$ is set to 3 while for horizontal plates and cylinders (or spheres) $\mathrm{n}$ is $7 / 2$ and 4 , respectively [47].

$$
N u^{n}=N u_{F}^{n} \pm N u_{N}^{n}
$$

A portion of the tank was bathed in sunlight which raised the tanks surface temperature. The solar energy incident on a surface consisted of direct and diffuse radiation. The part of solar radiation that reached the earth's surface without being scattered or absorbed by the atmosphere was referred to as direct solar radiation, $G_{\mathrm{D}}$. The diffuse radiation, $G_{\mathrm{d}}$, was assumed to reach the earth's surface uniformly from all directions. The total solar energy incident on a unit area of surface was then given by Equation 12 .

$$
G_{\text {solar }}=G_{D} \cos \theta+G_{d}
$$

The parameter $\theta$ was the angle of incidence that the sun's rays made with the normal of the surface [48]. Diffuse radiation varied from approximately 10 percent of the total radiation on a clear day to nearly 100 percent on a totally overcast day. In the formulation of the model all direct solar radiation was assumed to be normal to the surface and diffuse radiation was that of a clear day (approximately 10 percent of direct solar radiation). The net rate of radiation heat transfer to a surface exposed to solar and atmospheric radiation was then given by Equation 13.

$$
q_{\text {solar }}^{\prime \prime}=\alpha_{s} G_{\text {solar }}
$$


Where $\alpha_{s}$ was the surface absorptivity. Some typical values are shown in Table 3-1. Solar radiation data including global horizontal $\left(G_{\text {solar }}\right)$, direct normal $\left(G_{\mathrm{D}}\right)$ and diffuse normal $\left(G_{\mathrm{d}}\right)$ radiation are available by geographic location from the National Solar Radiation Database [49].

Table 3-1: Common Surface Absorptivity and Emissivity Values [47]

\begin{tabular}{|l|l|l|}
\hline Surface & Absorptivity $\left(\alpha_{s}\right)$ & Emissivity $(\varepsilon)$ \\
\hline Polished Aluminum & 0.09 & 0.03 \\
\hline Anodized Aluminum & 0.14 & 0.84 \\
\hline Aluminum Foil & 0.15 & 0.05 \\
\hline Polished Stainless Steel & 0.37 & 0.60 \\
\hline Dull Stainless Steel & 0.50 & 0.21 \\
\hline White Paint & 0.14 & 0.93 \\
\hline
\end{tabular}

As the temperature at the surface of the tank was unknown it can be found by constructing an energy balance equation. Assuming the inner surface of the tank was the same temperature as the contained LNG produced Equation 14.

$$
\frac{T_{\text {amb }}-T_{\text {surf }}}{R_{\text {conv }}}+\frac{T_{L N G}-T_{\text {surf }}}{R_{\text {Tank }}}+G_{\text {solar }} \alpha=0
$$

Rearranging allowed for the calculation of the surface temperature. Initially a surface temperature was assumed for the calculation of convection resistance and thermal conductivity properties. The new surface temperature may be different than the assumption. An iteration process was used to converge to more accurate values for the resistances and surface temperature. If the change in surface temperature was minimal $\left(\mathrm{dT}<0.1^{\circ} \mathrm{C}\right)$ then the process did not reiterate.

$$
T_{\text {surf }}=\left(-G_{\text {solar }} \alpha-\frac{T_{a m b}}{R_{\text {conv }}}-\frac{T_{L N G}}{R_{\text {Tank }}}\right)\left(\frac{R_{\text {Tank }} R_{\text {conv }}}{-R_{\text {Tank }}-R_{\text {conv }}}\right)
$$

The energy input was then calculated from the heat transfer through the equivalent resistance of the network.

$$
q=\frac{T_{a m b}-T_{L N G}}{R_{e q}}
$$

The internal convection of the tank was not addressed. Good correlations do not exist for the calculation of the internal convection inside a cryogenic tank and the phenomena of stratification is important to this process. See [50] for a full description on how stratification and internal convection can be handled in a vertical cryogenic tank. 


\subsubsection{Tank End Regions}

The top and bottom surfaces of the tank were modeled as a double-walled circular disk which was exposed to external convection, solar radiation on the outer surface, and communicated with methane fluid via convection on the inner surface. The provision for structural junctions between the inner and outer walls was included using the same area ratio $(\gamma)$ parameter. The wall section was represented by the thermal resistance network shown in Figure 3-3. Conduction through the tank wall was handled in the same manner as found in Equation 1. The individual thermal resistance equations are shown in Equations 17 - 20. All variables can be found in Figure 3-2.

$$
\begin{aligned}
R_{\text {cond }, \text { os }} & =\frac{t_{o}}{\pi r_{1}^{2} k_{m, 1}} \\
R_{\text {cond }, I S} & =\frac{t_{i}}{\pi r_{1}^{2} k_{m, 2}} \\
R_{\text {cond }, M L I} & =\frac{t_{\text {ins }}}{\pi r_{1}^{2} \gamma k_{M L I}} \\
R_{\text {cond,strut }}= & \frac{t_{\text {ins }}}{\pi r_{1}^{2}(1-\gamma) k_{\text {strut }}}
\end{aligned}
$$

Where $t_{o}$ was the thickness of the outer shell of the tank, $t_{i}$ was the thickness of the inner shell and $k_{\mathrm{m}}$ was the mean thermal conductivity of each shell layer in the equations. The thickness of the annulus between the inner and outer shell was denoted as $\mathrm{t}_{\mathrm{in}}$.

As in the previous region the outer surface was exposed to radiation and natural or forced convection with the atmosphere. Heat energy due to solar irradiation, $q_{\text {solar }}$ was applied at the outer surface of the tank in the same manner as found in the cylinder region. The convective resistance is shown in Equation 21.

$$
R_{\text {conv, air }}=\frac{1}{h_{o} A}=\frac{1}{\pi r_{1}^{2} h_{o}}
$$

Natural convection forming around the exterior of the tank at these regions was approximated as natural convection from an upward facing cold plate $\left(\mathrm{T}_{\mathrm{OS}}<\mathrm{T}_{\mathrm{amb}}\right)$ or downward facing cold plate in which case the empirical correlation of Equations 22 and 23 applied. Empirical correlations exist for forced convection over flat horizontal plates with mixed laminar and turbulent flow conditions. The standard correlations apply to rectangular plates of infinite width. Gartland and Winkelmann [51] addressed the use 
of the standard flat plate correlations for rectangular and circular horizontal geometries. Equations were offered for correlations for upward facing cooled $\left(\mathrm{T}_{\mathrm{OS}}<\mathrm{T}_{\mathrm{amb}}\right)$ roofs.

$$
\begin{gathered}
h_{o}=\eta \frac{k}{L_{n}} 0.27 R a_{L n}^{1 / 4}+\frac{k}{L_{e f f}} R_{f}\left(.664 \operatorname{Re}_{\text {Leff }}^{1 / 2} \operatorname{Pr}^{1 / 3}\right) \quad\left(L_{e f f}<X_{c}\right) \\
h_{o}=\eta \frac{k}{L_{n}} 0.27 R a_{L n}^{1 / 4}+\frac{k}{L_{e f f}} R_{f}\left[.037\left(\operatorname{Re}_{\text {Leff }}^{\frac{4}{5}}-\operatorname{Re}_{X c}^{\frac{4}{5}}\right)+.664 \operatorname{Re}_{X c}^{1 / 2}\right] \operatorname{Pr}^{1 / 3} \quad\left(L_{e f f} \geq X_{C}\right)
\end{gathered}
$$

By extension Equations 24 and 25 were used for downward facing cold plates.

$$
\begin{gathered}
h_{o}=\eta \frac{k}{L_{n}} 0.54 R a_{L n}^{1 / 4}+\frac{k}{L_{e f f}} R_{f}\left(.664 \operatorname{Re}_{\text {Leff }}^{1 / 2} \operatorname{Pr}^{1 / 3}\right) \quad\left(L_{e f f}<X_{C}\right) \\
h_{o}=\eta \frac{k}{L_{n}} 0.15 R a_{L n}^{1 / 3}+\frac{k}{L_{e f f}} R_{f}\left[.037\left(\operatorname{Re}_{\text {Leff }}^{\frac{4}{5}}-\operatorname{Re}_{X c}^{\frac{4}{5}}\right)+.664 \operatorname{Re}_{X c}^{1 / 2}\right] \operatorname{Pr}^{1 / 3} \quad\left(L_{e f f} \geq X_{C}\right)
\end{gathered}
$$

Where, in the equations, $\eta$ was the weighting factor for natural convection, $k$ was the thermal conductivity of air, $L_{n}$ was the characteristic length for natural convection $\left(L_{n}=D / 4\right), R a_{L n}$ was the Rayleigh number for natural convection, $L_{\text {eff }}$ was the effective length for forced convection, $R_{f}$ was a surface roughness factor, $\mathrm{Re}_{\mathrm{Leff}}$ was the Reynolds number based on the characteristic length for forced convection, $R e_{X c}$ was the Reynolds number at which transition from laminar to turbulent flow $\left(\mathrm{Re} \cong 5 \times 10^{5}\right)$ occurred, and Pr was the Prandtl number. Equation 22 applied when flow remained laminar and Equation 23 applied where transition from laminar to turbulent flow occured at a location $X_{c}$ along the circular plate. For a uniform distribution of wind directions, the effective length for a circular plate was $L_{\text {eff }}=0.81 D$ for laminar flow and $L_{\text {eff }}=0.82 D$ for turbulent flow where D was the outer diameter of the tank. In this study the surface roughness factor was taken as unity. The critical length at which the flow transitions from laminar to turbulent was given by Equation 26.

$$
x_{c}=5 \times 10^{5} \frac{\mu_{\text {air }}}{\rho_{\text {air }} U_{\infty}}
$$

Where $\mu$ was dynamic viscosity, $\rho$ was density and $U_{\infty}$ was the free stream wind velocity in the equation. The natural convection weighting factor was defined in terms of the Grashof and Reynolds numbers. 


$$
\eta=\frac{\ln \left(1+\frac{G r_{\text {Leff }}}{R e_{\text {Leff }}^{2}}\right)}{1+\ln \left(1+\frac{G r_{\text {Leff }}}{R e_{\text {Leff }}^{2}}\right)}
$$

Equations 22 and 23 assumed that natural and forced convection were additive but natural convection was suppressed as forced convection increases ( $\eta \rightarrow 0$ as Reynolds number becomes large).

\subsubsection{Stratification}

Stratification was handled by discretizing the tank. Layers were sliced into the vapor and liquid regions of the tank with each layer capable of evaluating a different temperature. Each layer was considered constant properties and uniform. Figure 3-4 shows an illustration of the layering, blue indicating the liquid region and red the vapor space.

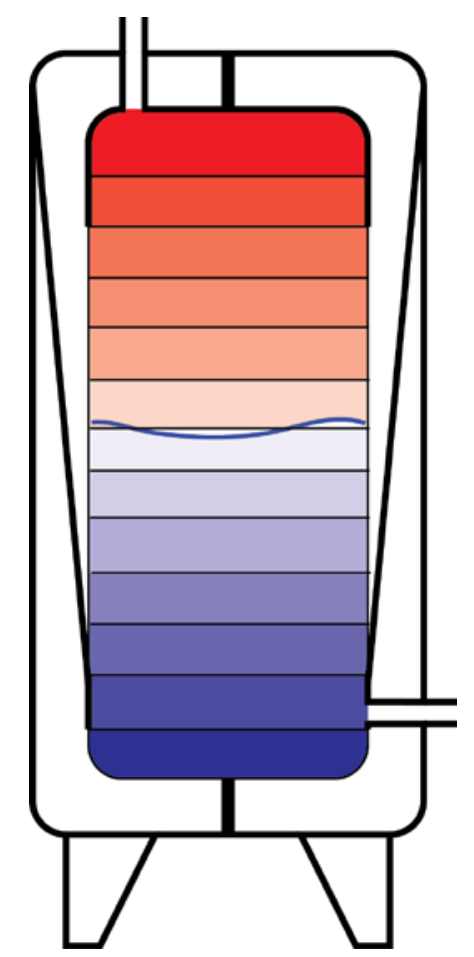

Figure 3-4: Illustration of Thermal Stratification Layers for Model

Layer thickness was not constant over the course of a simulation, and changed with the fill level. Each layer was an equal division of the region during the time step. The number of layers in each region was set prior to the start of the simulation. As the fill level changed so did the height of the layers in each region. Heat transfer to each layer was calculated based on the area of the layer exposed to the tank. Once heat transfer to the layers was calculated the information was then sent to the thermodynamic model. Only 
the stratified thermodynamic model found in [4] benefited from the stratified version of heat transfer through the tank wall because it used natural convection theory to calculate a temperature distribution.

\subsection{Dispense Piping Heat Transfer}

\subsubsection{Overview}

Calculation of the heat transfer into the dispense piping network required some simplifying assumptions. First, all the piping was considered in the horizontal orientation only. Second, many valves and fixtures were present in the lines of a station but their design specifics related to heat transfer were unknown. Valves and fixtures were approximated by an equivalent length of associated piping; this was done by measuring the length of the valve or fixture and treating it as an extension of the pipe it was connected to. In general there were three types of piping used at an LNG station. Bare (none insulated), foam insulated, and vacuum jacketed piping (VJP), which included Multi-layer insulated VJP (MLIP), were the types.

Another consideration was specific station heat management methods. For the dispense piping there were two main approaches used to handle the heat load. The station owner was observed to constantly keep the piping system cooled so the station would be ready to refuel whenever a vehicle arrived. Thus, the piping becomes a constant source of heat. However at an underutilized station the operator was observed to let the pipes warm up in the interim between fueling vehicles. The process was advantageous in this situation because eventually the thermal energy warmed the piping lines to ambient whereby no more heat was accrued through this source. Normally thermal energy reached the tank through the piping lines either by evaporation of LNG in the line returning to the tank or when LNG was recirculated to the tank. Once the piping warmed to ambient and after the LNG in the lines was evaporated back to the station tank the heat load was reduced. The dispense piping then contributed negligible heat to the station tank pressure rise. Once a vehicle arrived or normally called in ahead of time the station owner then cooled the piping down. The station which used the second strategy did not accrue the heat load of the pipes at all times but this was only possible because of the intermittent vehicle refueling. The first strategy (a well-utilized station) was assumed for the formulation of the heat transfer through the dispense piping.

The important assumptions are summarized as all the piping was considered in the horizontal orientation only. Valves and fixtures were approximated by an equivalent length of associated piping. The piping system was kept cooled and was a near constant source of heat load into the station tank, only varying

with ambient temperature. Energy transferred by conduction through the piping walls to the LNG inside was considered a direct heat load on the LNG in the station tank. 


\subsubsection{VJP and MLIP}

In a similar fashion to the development of the station tank heat transfer a resistance network approach was formulated for the three types of piping. The three types of pipe found in a fueling station were bare, foam insulated, VJP which includes MLIP. For VJP the heat leak was calculated by using Equation 28.

$$
Q=F_{e} F_{1-2} \sigma A_{1}\left(T_{2}^{4}-T_{1}^{4}\right)
$$

Where $F_{e}$ is the emissivity factor, $F_{1-2}$ is a configuration factor, $\sigma$ is the Stefan-Boltzmann constant, $\mathrm{A}_{1}$ is the area of inner surface, and $\mathrm{T}$ is the absolute temperature. In a completely enclosed vessel $\mathrm{F}_{1-2}=1$ and $\mathrm{F}_{\mathrm{e}}$ were calculated by using the emissivity of the surfaces $\left(\mathrm{e}_{1}\right.$ and $\left.\mathrm{e}_{2}\right)$ in Equation 29.

$$
\frac{1}{F_{e}}=\frac{1}{e_{1}}+\frac{A_{1}}{A_{2}}\left(\frac{1}{e_{2}}-1\right)
$$

MLIP represented the bulk of piping at an LNG fueling station. For MLIP the formulation was similar to the station tank because the piping uses spacers for support along with MLI. Inner spacers were know to be made of many materials but were assumed to be stainless steel in this analysis. The resistance network and diagram for MLIP is shown in Figure 3-5.
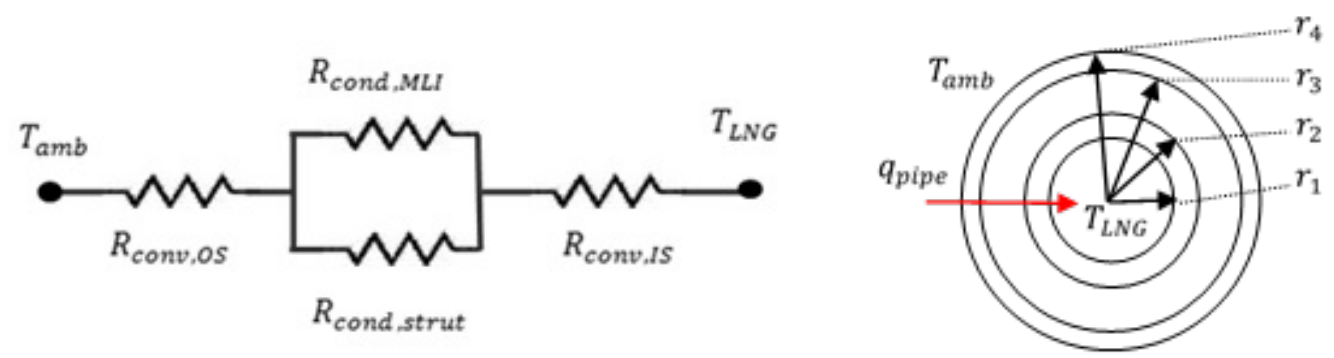

Figure 3-5: MLIP Diagram \& Thermal Resistance Network

Convection on the inner and outer surface of the MLIP was assumed negligible. The surface temperatures for the inner and outer portions of the tank was assumed to be the station tank LNG temperature and the ambient temperature, respectively. Table 3-2 shows some dimensions and heat leak information found in technical specifications for MLIP from Chart Industries [50], [52]. 
Table 3-2: MLIP Specifications From Industry Technical Specifications [50], [52]

\begin{tabular}{|l|l|l|l|}
\hline \multicolumn{2}{|l|}{ Inner Pipe } & Outer Pipe & \\
\hline Nominal Diameter & Actual Flow Diameter & Nominal Diameter & Static Heat Leak (W/m) \\
\hline $0.5 ”$ & $0.430 ”$ & $1.25 ”$ & 0.41 \\
\hline $1 ”$ & $1.185 ”$ & $2 ”$ & 0.46 \\
\hline $2 ”$ & $2.245 ”$ & $3 ”$ & 0.76 \\
\hline $3 ”$ & $3.334 ”$ & $5 ”$ & 1.04 \\
\hline
\end{tabular}

While the dimensions for the piping were available the thermal conductivity of the MLI in the pipe along with the amount and type of spacers used were not readily available. Quoted heat transfer values from industry information and recent research information about MLIP performance from Dye et al. [23] were used to find the remaining missing variables of thermal conductivity and area ratio. The MLI insulation was assumed constant while the heat leak values for the available diameters were used to calculate the amount of support material. The amount of support material was then kept constant for other piping sizes with only the dimensions being altered. Calculation of the heat load caused by MLIP was estimated by this procedure.

\subsubsection{Foam Insulated Piping}

Foam insulation piping consisted usually of a thick layer (3 to 4 inches) of low conductivity foam with an external metal foil to reflect solar loading. A resistance network was used for the two layers along with the proper thermal conductivity to calculate heat leak from foam piping. An industry provided value for the thermal conductivity of foam typically used was $0.0272 \mathrm{~W} / \mathrm{m}-\mathrm{K}$. Since the insulation had a low thermal conductivity external natural convection was not utilized in the heat transfer calculation and the surface temperature was assumed to be ambient temperature.

\subsubsection{Bare Pipe}

For bare piping a frost layer was observed to form when cryogenic fluid passed through the pipe altering the heat transfer rate to the LNG. This was a transient process with both the thickness and the thermal conductivity of the frost layer varying in time depending on local humidity conditions. In this analysis all air was considered dry air and therefore no frost layer was considered. In the resistance network for bare pipe the external natural convection resistance was not ignored. Exact mass flow rates of LNG were not available inside of the piping so no calculation of internal convection was used. Internal convection was assumed to be strong and the internal wall temperature to be that of the LNG.

Equation 30 was the correlation used for natural convection across a long horizontal cylinder. The equation is valid for $R a_{D} \leq 10^{12}$. 


$$
N u_{D}=\left\{.60+\frac{0.387 R a_{D}^{1 / 6}}{\left[1+(0.559 / P r)^{9 / 16}\right]^{8 / 27}}\right\}^{2}
$$

Wind speed values were not available for station locations, therefore, only natural convection was considered. An iterative process was used similar to that used for the station tank to calculate a more

accurate Nusselt number. The film temperature properties $\left(T_{\text {film }}=\frac{T_{a m b}+T_{\text {surf }}}{2}\right)$ were found, and used in an energy balance to find the surface temperature. The surface temperature was then used to find the new film temperature properties and the iteration continued. The iteration continued until a result was converged upon. If the change in surface temperature was minimal $\left(\mathrm{dT}<0.1^{\circ} \mathrm{C}\right)$ then the process did not reiterate.

\subsection{Dispenser Heat Transfer}

The station dispensers which fuel vehicles with LNG were another source of heat transfer into the LNG station system. Dispensers were difficult to address as much of the heat load produced was transient. Dispensers were only cooled before refueling a vehicle, and in the interim between fueling the dispensers warmed all the while approaching ambient temperature. Energy from the dispensers eventually reached the tank by either the cool down process itself or through evaporated/warmed LNG returned to the station tank. In order to obtain a calculation of the heat transfer, the dispenser was treated as an assemblage of horizontal piping, similar to the piping system, and the temperature rise in the dispenser was estimated. During the cool down process the energy returned to the tank was calculated by Equation 31.

$$
Q_{d i s p}=\frac{1}{2} Q_{s s} t_{s S}+\sum m_{w} C_{w}\left(T_{o}-T_{s S}\right)
$$

Where Qss was the steady state heat transfer rate, $\mathrm{tss}_{\mathrm{s}}$ was the cool down time, $\mathrm{m}_{\mathrm{w}}$ was the mass of piping, $C_{w}$ was the average specific heat of the pipe, $T_{o}$ was the initial temperature of the pipe, and $T_{s s}$ was the steady state temperature or LNG fluid temp from the station tank.

The temperature change of the dispensers was modeled by using an energy balance approach. All piping and fixtures were considered at a uniform temperature. Piping inside the dispenser had an associated mass of stainless steel and an associated amount of LNG contained within the piping. Assuming that the pipe wall was always the same temperature as that of the LNG, Equations 33 and (33) were used as a basis for the temperature tracking. Both the pipe wall material and the LNG were used to determine an energy balance.

$$
\begin{gathered}
\Delta E=Q_{\text {disp }}=m_{L N G} H_{\text {vap }} \\
\Delta E=Q_{\text {disp }}=\left(m_{\text {pipe }} C_{\text {p,pipe }}+m_{L N G} C_{p, L N G}\right)\left(T_{\text {disp }}^{n}-T_{\text {disp }}^{n-1}\right)
\end{gathered}
$$


In the algorithm used the LNG inside the lines was allowed to vaporize utilizing Equation (32). Once mass of LNG gained enough energy to completely vaporize the calculation utilized Equation (33) which included the mass of methane vapor, the mass of the pipe wall material and associated specific heats. The temperature of the dispenser in the current time step was denoted by $T_{\text {disp }}^{n}$ while $T_{\text {disp }}^{n-1}$ was the temperature of the dispenser at the previous time step. The heat transferred through the dispenser piping was calculated by Equation 34 and used in the energy balance equation.

$$
Q_{\text {disp }}=\frac{T_{a m b}-T_{\text {disp }}^{n-1}}{R_{\text {disp,pipe }}}
$$

A new dispenser temperature was solved for by combining Equation (32) and 34. The new temperature was then used in the calculation of $\mathrm{Q}_{\text {disp }}$ in the next time step. Eventually the temperature stabilized at ambient conditions until a new vehicle refueling event occurred.

\subsection{Offload Piping Heat Transfer}

Stations were refilled by tankers through the offload pump piping. Experience from the LNG stations visited during the study showed the offload pump as a source of heat input to the station tank which was transient. LNG contained in the lines after a refill warmed and expanded into the tank. Eventually the offload piping and methane in the lines reached ambient temperature. Expansion of LNG vapor into the tank was controlled by a one way check valve. The dynamics for the expansion of LNG into the station were difficult to determine. Data on the pressure rise, temperature, and dimensions of the offload pump lines were available for the first station visited in the study and therefore an estimate of the energy transfer from this source was estimated. For the second station this information was not available, and, therefore, was omitted.

For the calculation of the offload pump energy transfer the amount of LNG contained within the piping was calculated using the piping geometry to calculate the volume of the lines. Using the initial state of the fluid in the line (found from experimental data) the mass of LNG in the lines was estimated. Next using property values for methane the difference between the internal energy of the first state and final state of fluid in the lines was found. The difference was used as the total amount of energy which entered the tank. In the station data, pressure in the lines followed the station pressure until the methane in the lines no longer could force anymore vapor through the one way valve. The time period over which this occurred was calculated, and used to determine an average rate of heat leak into the station tank by dividing the total energy by this time period. 


\subsection{Environmental Inputs}

Environmental conditions around the audited LNG stations were taken into account by using values collected by the National Oceanic and Atmospheric Administration (NOAA). For experiments conducted at CAFEE, temperature and solar loading data were collected locally. Ambient temperature values from weather stations close to the LNG stations investigated were used in the audit stations which were provided by NOAA. Accuracy of the temperature values depended upon the specific weather station. Based on recent studies at least 80 percent of weather stations have a best case temperature accuracy of $2^{\circ} \mathrm{C}$ [53], [54]. Therefore, most likely the temperature data used for the energy transfer calculations suffered from at least a $2{ }^{\circ} \mathrm{C}$ uncertainty in the temperature.

Wind and solar radiation values were also sometimes available through NOAA. Wind values were not generally used in the model although equations were provided for its incorporation. Solar radiation data for the times of the study were not yet available from NOAA, and, therefore, no current solar radiation values were used in the comparison. A set variation of solar radiation was used instead as an input for sunlight incident on the tank surface for the station comparison simulations. Figure 3-6 shows the assumed incident solar radiation. The input was the positive portion of a sinusoidal wave peaking at $980 \mathrm{~W} / \mathrm{m}^{2}$ for a duration of 12 hours. The profile is based on solar loading data collected at WVU.

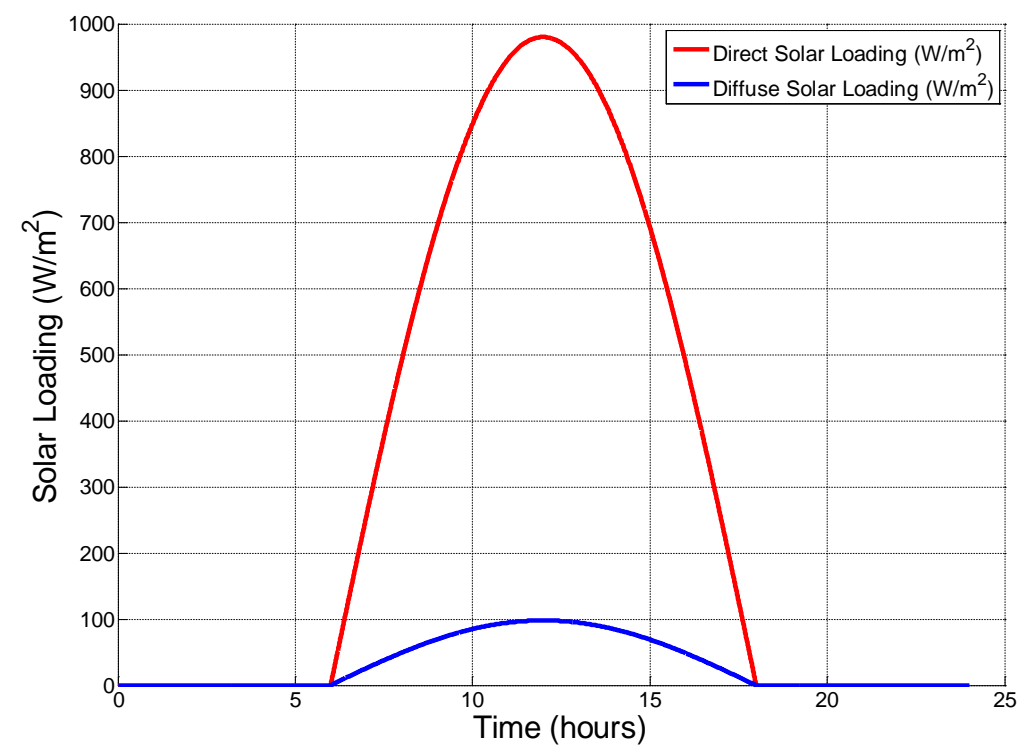

Figure 3-6: Assumed Daily Solar Loading Profile for Audit Station Comparions 


\subsection{Thermodynamics and Vent Module}

The thermodynamic model was used to determine the state and properties of the LNG stored in the cryogenic tank. Varying mass flow of LNG into and out of the tank was also accounted for by the thermodynamic model. The modeled accounted for and employed energy balance, mass balance, thermodynamic property relations, and property data to describe the change in the LNG liquid and vapor over time. Two different approaches to the thermodynamics were created, referred to as the homogeneous approach and the stratified approach. In addition, a vent module was created base on the homogeneous thermodynamic approach. These approaches were develop by Sandoval and detailed in [4].

Assumptions were made to the thermodynamics to create an effective and efficient model. First the LNG composition was considered 100\% methane $\left(\mathrm{CH}_{4}\right)$. The effects of other constituents such as ethane, propane, butane, and carbon dioxide were not considered. Next, heat transfer in the fluid was assumed to not be great enough to cause boiling. Evaporation or condensation of LNG only occurred at the surface of the liquid-vapor interphase. In addition, the process of evaporation at the vapor-liquid interface was considered in quasi-equilibrium. Fast occurring transient behaviors were not considered. Pressure was assumed uniform in the tank. The kinetic and potential energy associated with LNG entering and exiting the tank was neglected. All LNG liquid or vapor exiting the tank was considered saturated. For the homogeneous approach the temperature inside the tank was considered uniform with saturated liquid and vapor.

\subsubsection{Saturated Homogeneous Thermodynamic Approach}

The homogeneous thermodynamics approach treated the liquid and vapor inside the tank as one saturated system. Three concepts were implemented to determine the state of the tank. The first was conservation of mass, equation 35, and the second was energy balance, equation 36.

$$
\begin{gathered}
\frac{\mathrm{dm}}{\mathrm{dt}}=\sum \dot{\mathrm{m}}_{\text {in }}-\sum \dot{\mathrm{m}}_{\text {out }} \\
\frac{\mathrm{dU}}{\mathrm{dt}}=\dot{\mathrm{Q}}+\sum \dot{\mathrm{m}}_{\mathrm{in}} \mathrm{h}_{\text {in }}-\Sigma \dot{\mathrm{m}}_{\text {out }} \mathrm{h}_{\text {out }}
\end{gathered}
$$

In equations 35 and 36 the total heat transfer rate was denoted by $\dot{Q}$, and was calculated by the heat transfer model. In addition, the mass flow rate was denoted by $\dot{m}$, the enthalpy was denoted by h, the internal energy was denoted by U, and the subscripts "in" and “out” represented entering and exiting of the tank. Properties were determined by using saturated thermodynamic property tables for methane using pressure and quality.

The third concept required an iterative approach to determine the new pressure in the tank for each time step. First, the new specific internal energy was calculated by the energy equation and the new specific volume was calculated through the mass balance equation. Next a range of pressures was chosen from the 
property data around the previous time step's pressure. A quality value for specific volume and specific internal energy was then calculated using the property data from the range of pressures and the new average internal energy and average specific volume. The pressures (and associated properties) were changed until the two quality values nearly equaled one another with only a small relative error $\varepsilon=10^{-6}$. After the error condition was met the new pressure and properties were found and passed on to the rest of the model.

\subsubsection{Stratified Thermodynamic Approach}

The stratified thermodynamic approach split the tank into two regions, the liquid and vapor regions. In the two regions it calculated the temperature profiles by using the turbulent boundary layer equations for a cylindrical tank. The profiles were then used by the heat transfer model to calculate a more accurate heat leak. A derivation of the model can be found in [4].

The important assumptions for the model were as follows. Heat transferred through the tank walls to the LNG was carried upward to the top of each region by the natural convection boundary layer. The initial temperature and conditions in the tank was set as thermodynamically saturated. Pressure was determined by the temperature of the liquid at the liquid-vapor interface. In the derivation the thickness of the temperature and velocity profiles was assume to be equal, since pure methane's Prandtl number was close to one.

\subsubsection{Venting Module}

The LNG tank venting model calculated the mass of vapor that needed to be removed from a LNG tank in order to drop the pressure from an initial pressure to a final pressure. The model assumed isothermal homogenous conditions within the tank during this venting process. Additionally, potential and kinetic energy, work, and heat transfer were neglected. The venting module was the component of the LNG station model used to estimate the mass of vapor that was transferred from a vehicle tank to the station tank during vapor balancing. It was also used to estimate the mass of vapor released from the PRV in the event that the PRV activation pressure was reached. The development and the accuracy assessment of the module can be found in [4].

\subsection{Model Overview}

The basic formulation of the energy transfer model to calculated energy input into the tank LNG through the tank wall was presented. To illustrate the approach, first, a vertically oriented bulk storage tank was considered. A similar approach was applied to a horizontally oriented storage tanks. The specific equations and empirical correlations for horizontal tanks differed from those presented above but the same approach was utilized. The additional equations are found in appendix A. In addition, equations and 
approaches were presented for the calculation of energy transfer through other components of a LNG fueling station. The components included the dispense piping, the offload piping, and the fuel dispenser.

The overall model had many modules which worked in unison to create both a hold time test model and a full LNG station model. Figure 3-7 shows the flowchart for the modules and the input/outputs of each. MATLAB/Simulink was used to create the model.

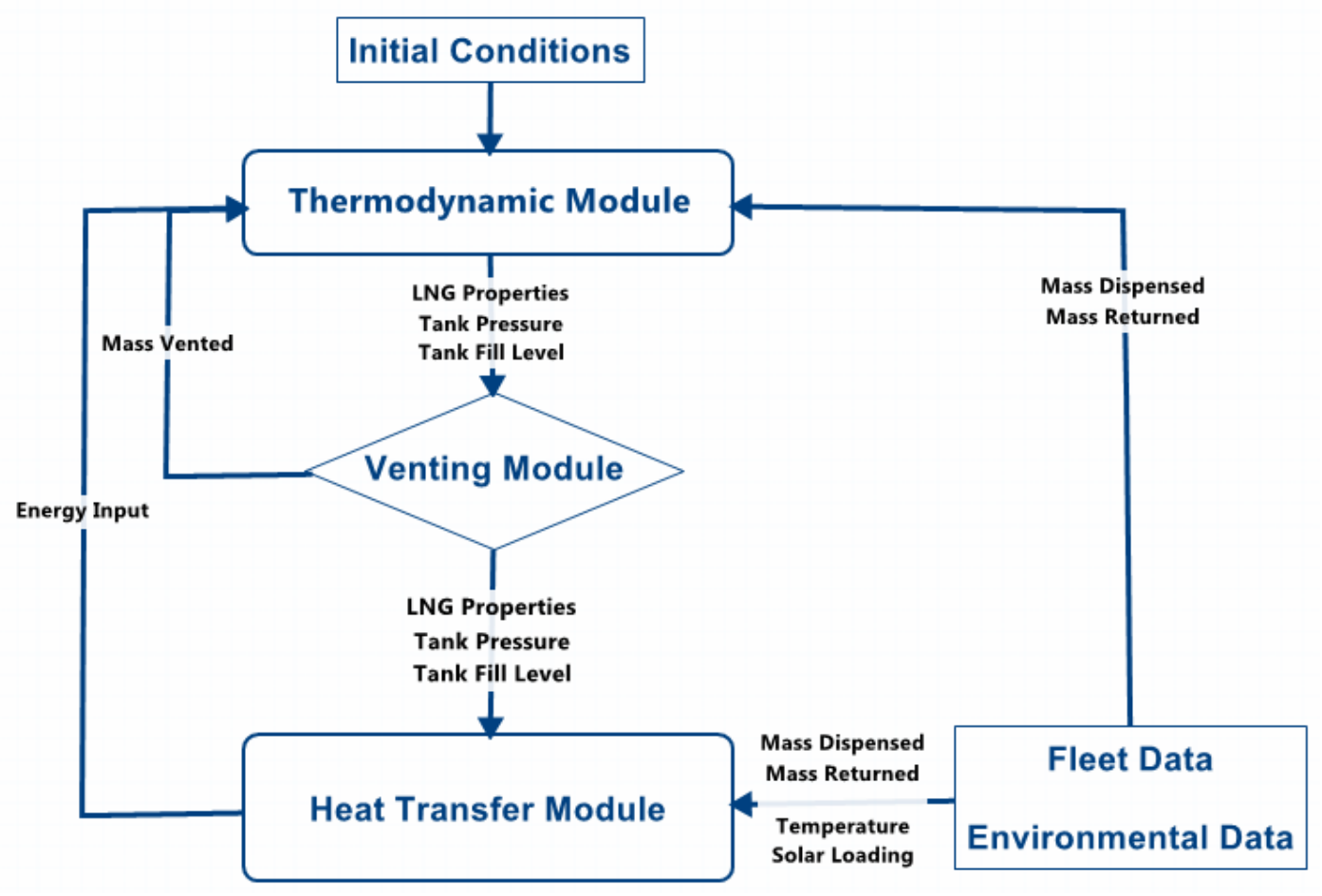

Figure 3-7: LNG Station Model Flow Chart

The model started by receiving initial conditions into the thermodynamics module. Initial conditions included tank pressure, mass of LNG inside the storage tank, and necessary dimensions. Fluid properties such as temperature, pressure, density, specific internal energy, and specific enthalpy were calculated by the thermodynamic module. In addition, mass balancing was handled by the module. As discussed earlier the thermodynamic module utilized one of the two approaches for each simulation. The properties of the working fluid were passed from the thermodynamic model to the venting module. If the pressure was above the vent pressure set for the system the venting module would activate. The module calculated the mass vented based on an initial assumption to vent down to a predetermined tank pressure. The fluid properties then passed to the energy transfer module. Environmental conditions and fleet behavior were also given to the energy transfer model. Once the energy transfer module calculated the heat load and passed it to the thermodynamic module then next iteration occurred. In this way the pressure was calculated time step by time step. 


\section{Data Collection and Model Comparison}

Validation of the model and its modules was completed by comparing to data collected from various sources. Normal evaporation rate (NER) experiments, hold time experiments of two LNG vehicle tanks, and audits of two operational LNG stations were conducted to obtain data for the model creation and validation. The NER experiments were conducted to characterize the heat leak into two vehicle tanks by measuring the amount of methane boiled off from the tank. Hold time experiments tracked the pressure rise of the stationary vehicle tanks at various fill levels until venting occurred. Both the hold time tests and NER experiments were used primarily for insight into the thermodynamic approaches and behavior of the tank heat transfer. Audits of two LNG stations were completed and the data used to create a full LNG station model.

\subsection{Hold Time Experiments}

\subsubsection{Data Collection}

Hold time experiments were conducted on two different types of LNG vehicle tanks. One tank was without an internal fluid pump (IFP), or a NON-IFP tank, while the other utilized an IFP. Each experiment collected data for time, tank surface temperature (top \& bottom), tank solar loading (top \& bottom), ambient temperature, tank pressure, and tank weight. Data were continuously collected for the duration at a rate of $1 \mathrm{~Hz}$. The experiments were hold time tests of static tanks, and the internal fluid pump was not powered at any time. Figure 4-1 shows a picture of the experimental setup. The setup was exposed to local environmental conditions for the duration of the experiments.

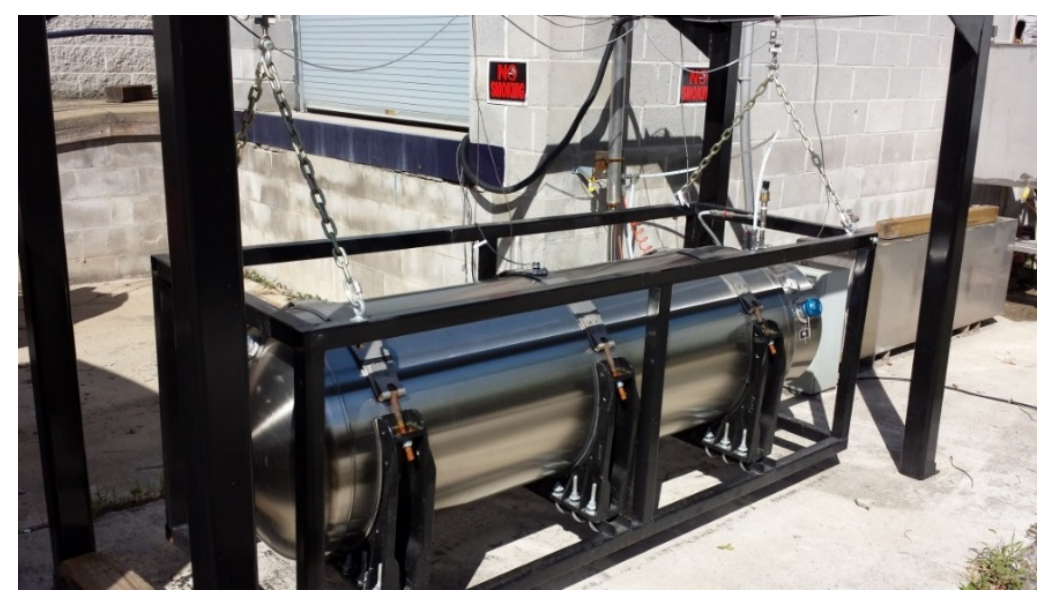

Figure 4-1: Vehicle Tank Test Stand 
A total of 14 experiments were run on both tanks; nine on the NON-IFP tank and five on the IFP tank. Pressure was measured from a pressure transducer with a range of $0 \mathrm{kPa}$ to $3450 \mathrm{kPa}$ (0-500 psia) with an uncertainty of +/- $8.62 \mathrm{kPa}$ (+/-1.25 psia). Figure 4-2 shows the pressure rise for the NON-IFP tank over experiment 2. Each tank was equipped with a factory installed pressure relief valve (PRV). Experiments were run until the PRV fully opened. The PRV was set to release pressure at $1690 \mathrm{kPa}$ (245 psia), down to a lower pressure, and then shut off. Most experiments the tank vented nominally at the set pressure. In a few experiments the tank slowly vented or bled off pressure below the $1690 \mathrm{kPa}$ (245 psia) set point. The jagged end portion of the data in Figure 4-2 was typical for PRV venting behavior.

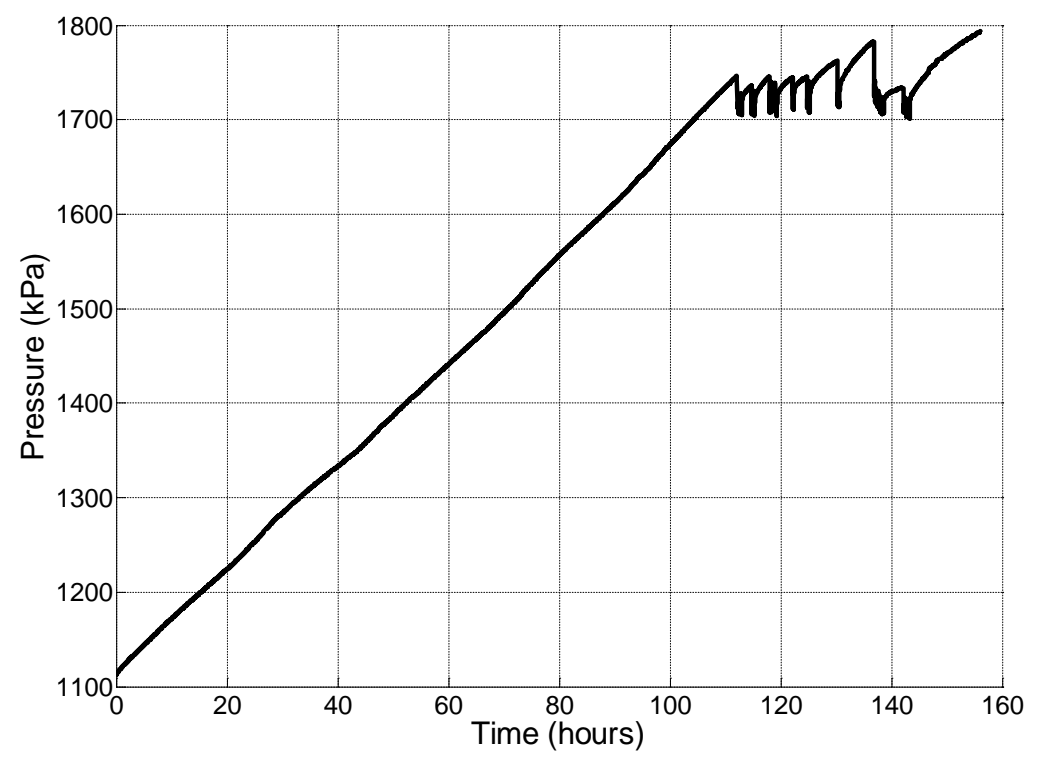

Figure 4-2: NON-IFP Tank Hold Time Test 2 - Tank Pressure Rise Trend with PRV Historesis

As seen in Figure 4-1 two chains suspended the cryogenic tank. Each chain was attached to a load cell used for total mass measurement. The load cells had an accuracy of $+/-0.03 \%$ of full scale output (FSO), and were $680 \mathrm{~kg}$ (1500 lbs.) sensors. The corresponding uncertainty of both sensors was +/-0.41 kg (+/- .90 lbs.). Fill level was calculated by using the weight measurement from the experimental setup. The actual weight was subtracted from the dry weight to calculate the mass of LNG inside the tank. A theoretical maximum amount of mass capable of storage in the tank was calculated by multiplying the quoted volumes of the tanks by the density of liquid methane at 1 atmosphere. Fill level was then calculated by dividing the two mass amounts. Figure 4-3 shows the weight of LNG for the NON-IFP tank in experiment 2. The oscillations in the figure were likely caused by precipitation and dew settling on the tanks. Effects from precipitation and dew accumulation were not quantified, but the oscillations possibly caused by this were 
within the uncertainty of the sensors. The average weight value was used for calculation purposes during the oscillating period.

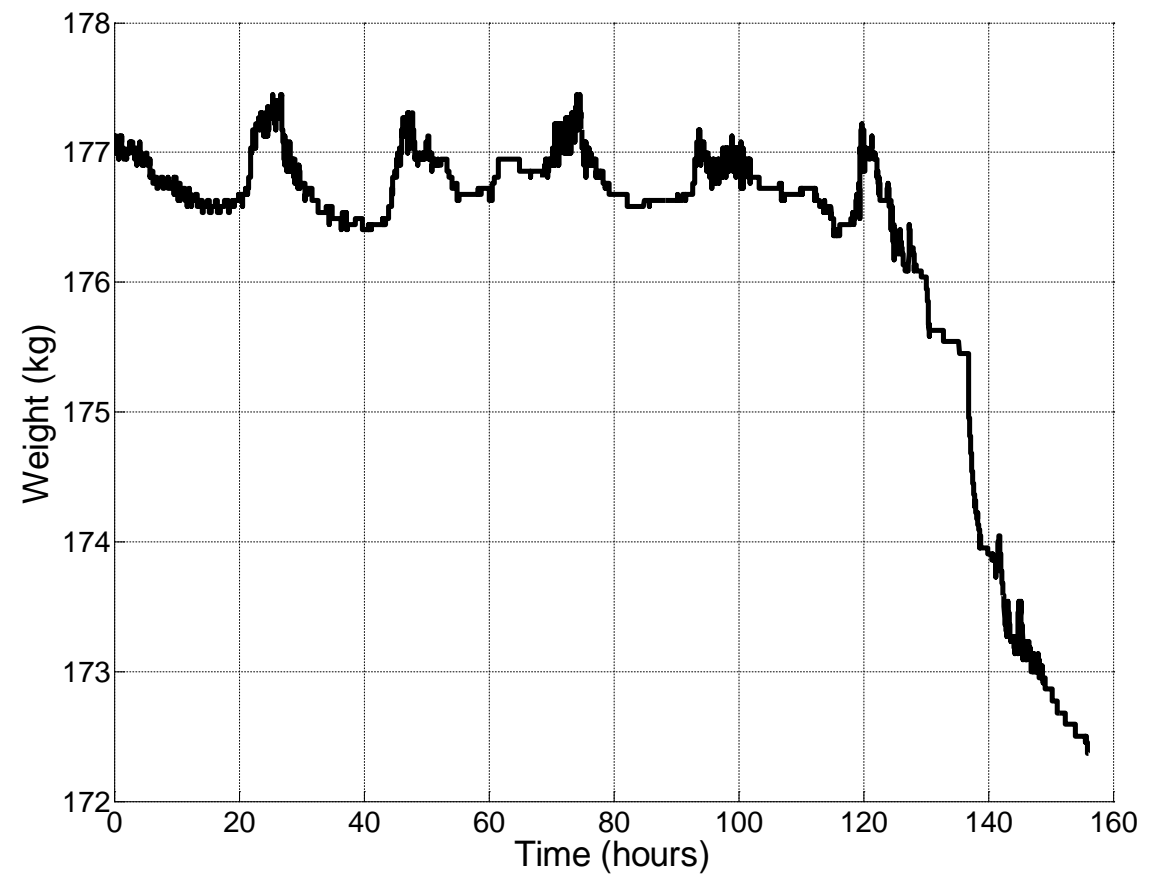

Figure 4-3: NON-IFP Tank Hold Time Test 2 - Tank Weight Used to Calculate Fill Level

The NON-IFP tank had a volume of 561 liters and could contain 230 kilograms of LNG (at atmospheric pressure). The IFP tank had a volume of 450 liters and could contain 180 kilograms of LNG. Table 4-1 shows the industry quoted dimensions for the two tanks.

Table 4-1: Pressure Rise Experiment Tank Dimensions

\begin{tabular}{|l|l|l|}
\hline Tank Type & \multicolumn{1}{|c|}{ NON-IFP Tank } & \multicolumn{1}{c|}{ IFP Tank } \\
\hline Diameter (mm) & 660 & 660 \\
\hline Length (mm) & 2285 & 2160 \\
\hline Net Volume (ltr) & 511 & 405 \\
\hline Gross Volume (ltr) & 561 & 450 \\
\hline Empty Mass (kg) & 285 & 409 \\
\hline
\end{tabular}

Local ambient temperature, surface temperature of the tank, and solar loading data were also collected. Two type K thermocouples attached to the top and bottom of the tank collected data for the surface temperature of the tanks. Another type $\mathrm{K}$ thermocouple was used to measure local ambient 
temperature. Each thermocouple had an accuracy of $2.2^{\circ} \mathrm{C}$ or $0.75 \%$ FSO. Ambient temperature data are shown in Figure 4-4.

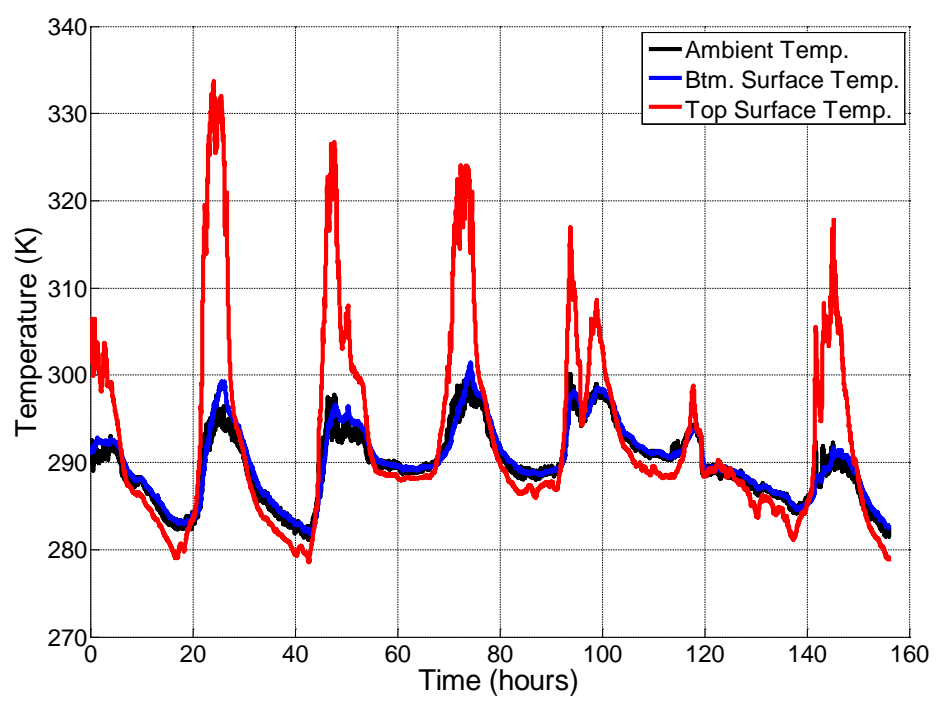

Figure 4-4: NON-IFP Tank Hold Time Test 2 - Ambient Temperatures and Surface Temperatures for Hold Time Experiment

Solar radiation loading was measured by two solar cells, a Campbell Scientific LI-200X and a WVU Solar Cell Calibrated to the LI-200X, both with an accuracy of $+/-3 \%$. One was placed on top of the tank while the other was placed on the bottom of the tank. Solar radiation increased the temperature of the surface as found in Figure 4-4. Figure 4-5 shows the solar loading (Watts $/ \mathrm{m}^{2}$ ) data for the second experiment of the NON-IFP tank.

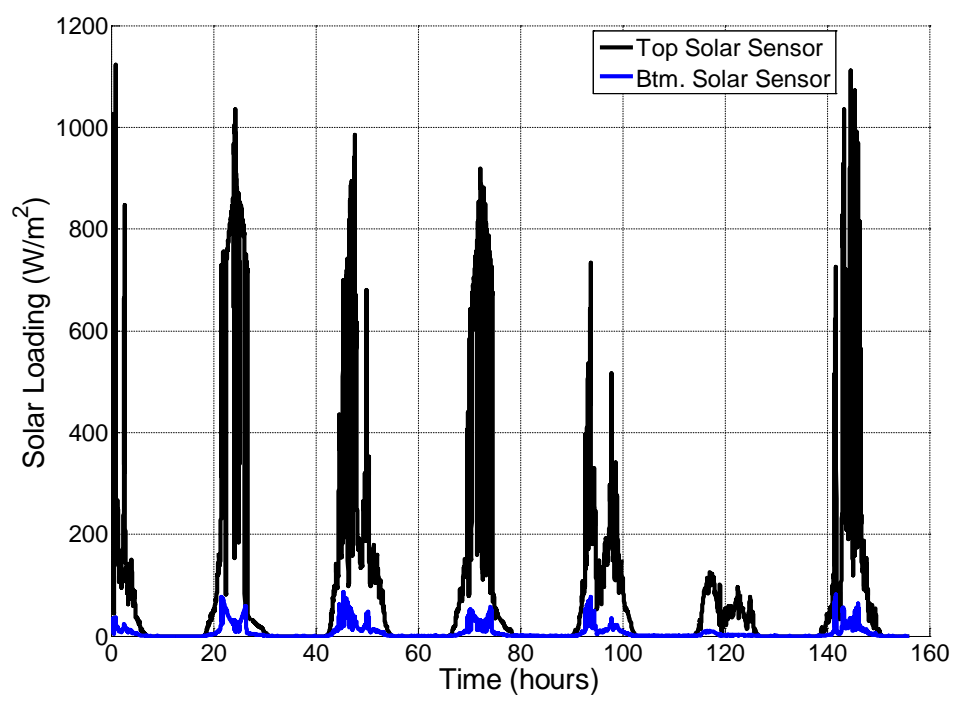

Figure 4-5: NON-IFP Tank Hold Tim Test 2 - Solar Loading Incident on Tank Surfaces 
Each experiment collected the described data for its duration. Table 4-2 shows a summary of the data collected from the two vehicle tanks. The experiments were conducted over the course of six months from $9 / 10 / 13$ to $3 / 31 / 14$.

Table 4-2: IFP \& NON-IFP Tank Pressure Rise Summary

\begin{tabular}{|c|c|c|c|c|c|c|}
\hline Exp. & $\begin{array}{l}\text { Duration } \\
\text { (hrs.) }\end{array}$ & $\begin{array}{c}\text { Fill } \\
\text { Fraction }\end{array}$ & $\begin{array}{l}\text { Avg. } \\
\text { Temp. } \\
\text { (K) }\end{array}$ & $\begin{array}{l}\text { Avg. Solar Loading } \\
\left(\mathrm{W} / \mathrm{m}^{2}\right)\end{array}$ & $\begin{array}{l}\text { Initial Pressure } \\
\qquad(\mathrm{kPa})\end{array}$ & $\begin{array}{l}\text { Peak Pressure } \\
\quad(\mathrm{kPa})\end{array}$ \\
\hline \multicolumn{7}{|c|}{ NON-IFP Tank Experiments } \\
\hline 1 & 68.64 & 1.05 & 292.2 & 98.8 & 1255.5 & 1669.2 \\
\hline 2 & 156.08 & 0.88 & 289.7 & 94.6 & 1114.2 & 1794.0 \\
\hline 3 & 386.75 & 0.52 & 290.1 & 76.5 & 137.9 & 1799.5 \\
\hline 4 & 297.29 & 0.31 & 284.9 & 70.2 & 137.9 & 1565.1 \\
\hline 5 & 550.08 & 0.72 & 276.2 & 39.9 & 137.9 & 1749.9 \\
\hline 6 & 538.71 & 0.45 & 275.8 & 23.5 & 137.9 & 1964.3 \\
\hline 7 & 1252.10 & 0.28 & 271.5 & 22.5 & 137.9 & 1804.4 \\
\hline 8 & 51.63 & 0.10 & 265.9 & 2.8 & 137.9 & 223.4 \\
\hline 9 & 237.66 & 0.65 & 284.9 & 95.4 & 137.9 & 790.1 \\
\hline \multicolumn{7}{|c|}{ IFP Tank Experiments } \\
\hline 1 & 36.08 & 1.04 & 289.3 & 13.6 & 1678.2 & 1773.3 \\
\hline 2 & 382.74 & 0.54 & 274.5 & 27.9 & 137.9 & 1820.2 \\
\hline 3 & 410.74 & 0.25 & 272.2 & 19.1 & 137.9 & 1791.9 \\
\hline 4 & 788.24 & 0.14 & 272.9 & 37.9 & 137.9 & 1443.1 \\
\hline 5 & 348.49 & 0.35 & 281.3 & 90.5 & 137.9 & 1634.1 \\
\hline
\end{tabular}

The first experiment for each tank appeared to be at an overfilled state when calculating the fill level. This occurred after filling the tank from an empty and ambient temperature state. Each tank was filled at a commercial LNG station. During the first tank filling the hot tank warmed the LNG coming into the tank, spiking the pressure above the maximum dispensing pressure quickly. Thus, a small amount of LNG made it into the vehicle tank from the first fill. The vehicle tank was then vented back to the station tank to reduce pressure and allow for more LNG to be dispensed. As many as 5 iterations of this process were required to fully fill the tank. After the tank was returned to the experimental apparatus the measured weight indicated an overfill condition. Normally, a tank cannot be filled above the 90\% mark as a dedicated ullage (vapor) space was present in the tank, installed by the manufacture. The ullage space served as expansion room for the cryogenic fluid as it warmed and was present on all LNG tank designs for safety reasons. Subsequent refilling of the tanks did not produce the same effect. The ullage space may have been filled on these initial fills resulting in the overfilled state. In addition the tank may be slightly bigger than manufacture 
specifications which were used for the calculation of fill level. These experiments were excluded from the model comparison.

Experiments 5 through 7 of the NON-IFP tank were oscillated for 60 seconds every hour by a small electric motor to determine if the oscillation had an effect on the hold time of the tank. These were used in the comparison of the two thermodynamic models against the experimental hold time test data.

As previously explained the experiments were effected by external weather conditions. These conditions included direct exposure to precipitation. Experiments 5, 6, 7, and 8 of the NON-IFP tank were exposed to snow conditions. Accumulated snow on the tank surfaces occurred for an extended period of time (1 - 2 days) for experiments 7 \& 8. For all the IFP tank experiments snow conditions were present during the data collection with accumulation occurring on experiments 2 through 5.

\subsubsection{Model Comparison}

The model was compared to the experimental data through pressure rise. Table 4-3 shows the model results and error in terms of $\mathrm{dP} / \mathrm{dt}$ of each approach.

Table 4-3: Tank Hold Time Pressure Rise Rate Comparison

\begin{tabular}{|c|c|c|c|c|c|c|c|c|}
\hline \multirow{2}{*}{ Exp. } & \multirow{2}{*}{$\begin{array}{l}\text { Duration } \\
\text { (hrs.) }\end{array}$} & \multirow{2}{*}{$\begin{array}{c}\text { Fill } \\
\text { Fraction }\end{array}$} & \multirow{2}{*}{$\begin{array}{c}\text { Initial } \\
\text { Pressure } \\
(\mathrm{kPa})\end{array}$} & \multicolumn{3}{|c|}{$\begin{array}{c}\text { Final Pressure } \\
(\mathrm{kPa})\end{array}$} & \multicolumn{2}{|c|}{$\begin{array}{c}\mathrm{dP} / \mathrm{dt} \text { Error } \\
(\%)\end{array}$} \\
\hline & & & & Exp. & Homog. & Stratified & Homog. & Stratified \\
\hline \multicolumn{9}{|c|}{ NON-IFP Tank } \\
\hline 1 & 68.6 & 1.05 & 1255 & & & & & \\
\hline 2 & 93.8 & 0.88 & 1114 & 1634 & 1696 & 1745 & $11.9 \%$ & $21.3 \%$ \\
\hline 3 & 264.5 & 0.52 & 138 & 1131 & 1800 & 920 & $67.3 \%$ & $-21.2 \%$ \\
\hline 4 & 172.3 & 0.31 & 138 & 832 & 1567 & 521 & $105.9 \%$ & $-44.8 \%$ \\
\hline 5 & 373.5 & 0.72 & 138 & 1155 & 1752 & 1370 & $58.7 \%$ & $21.1 \%$ \\
\hline 6 & 302.3 & 0.45 & 138 & 1038 & 1965 & 849 & $103.0 \%$ & $-21.0 \%$ \\
\hline 7 & 215.5 & 0.28 & 138 & 428 & 1805 & 548 & $474.8 \%$ & $41.4 \%$ \\
\hline 8 & 12.3 & 0.10 & 138 & 157 & 222 & 155 & $342.1 \%$ & $-10.5 \%$ \\
\hline 9 & 175.8 & 0.65 & 138 & 553 & 790 & 590 & $57.1 \%$ & $8.9 \%$ \\
\hline \multicolumn{9}{|c|}{ IFP Tank } \\
\hline 1 & 36.1 & 1.04 & 1678 & & & & & \\
\hline 2 & 266.5 & 0.54 & 138 & 1229 & 1820 & 977 & $54.1 \%$ & $-23.1 \%$ \\
\hline 3 & 158.5 & 0.25 & 138 & 759 & 1794 & 516 & $166.6 \%$ & $-39.1 \%$ \\
\hline 4 & 98.5 & 0.14 & 138 & 264 & 1381 & 342 & $886.5 \%$ & $61.9 \%$ \\
\hline 5 & 175.3 & 0.35 & 138 & 763 & 1634 & 605 & $139.3 \%$ & $-25.3 \%$ \\
\hline
\end{tabular}

Environmental conditions and initial conditions were used by the model to calculate pressure. Ambient temperature and solar loading data collected by the experiments was used by the heat transfer module to calculate the heat leak into the tank. Initial pressure and fill level were used to obtain an initial 
thermodynamic state by the model. Two thermodynamic approaches were compared both of which utilized the one heat transfer model. Pressure rise was then calculated by the model and compared to the experimentally measured pressure rise.

Pressure rise rate error was calculated by Equation 37 where the final model pressure was $\mathrm{P}_{\mathrm{f}}$, model, the final experimental pressure was $\mathrm{P}_{\mathrm{f} \text {, exp., }}$ the initial pressure was $\mathrm{P}_{\mathrm{i}}$, and $\mathrm{t}$ was the duration.

$$
\text { error }=\left(\frac{P_{f, \text { model }}-P_{i}}{t}-\frac{P_{f, \text { exp. }}-P_{i .}}{t}\right) / \frac{P_{f, \text { exp. }}-P_{i .}}{t}
$$

Table 4-4 used only the final pressures of the tank as comparison and for calculation of error.

Table 4-4: Tank Hold Time Final Pressure Comparison

\begin{tabular}{|r|r|r|r|r|r|}
\hline Exp. & \multicolumn{3}{|c|}{ Final Pressure (kPa) } & \multicolumn{2}{c|}{ Error (\%) } \\
\hline \multicolumn{7}{|c|}{ NON-IFP Tank Experiments } \\
\hline 1 & Exp. & Homog. & Stratified & Homog. & Stratified \\
\hline 2 & 1634.1 & 1696.8 & 1745.1 & $3.8 \%$ & $6.8 \%$ \\
\hline 3 & 1131.4 & 1800.2 & 920.5 & $59.1 \%$ & $-18.6 \%$ \\
\hline 4 & 832.9 & 1567.9 & 521.2 & $88.2 \%$ & $-37.4 \%$ \\
\hline 5 & 1155.6 & 1752.0 & 1370.7 & $51.6 \%$ & $18.6 \%$ \\
\hline 6 & 1038.4 & 1965.0 & 849.4 & $89.2 \%$ & $-18.2 \%$ \\
\hline 7 & 428.2 & 1805.0 & 548.8 & $321.5 \%$ & $28.2 \%$ \\
\hline 8 & 157.2 & 222.0 & 155.1 & $41.2 \%$ & $-1.3 \%$ \\
\hline 9 & 553.6 & 790.1 & 590.2 & $42.7 \%$ & $6.6 \%$ \\
\hline \multicolumn{7}{|c|}{} & IFP - Tank & & \\
\hline 1 & 1229.3 & 1820.2 & 977.7 & $48.1 \%$ & $-20.5 \%$ \\
\hline 2 & 759.8 & 1794.7 & 516.4 & $136.2 \%$ & $-32.0 \%$ \\
\hline 3 & 264.1 & 1381.7 & 342.0 & $423.1 \%$ & $29.4 \%$ \\
\hline 4 & 763.9 & 1634.1 & 605.4 & $113.9 \%$ & $-20.8 \%$ \\
\hline 5 & & & & & \\
\hline
\end{tabular}

The duration value found in Table 4-3 was usually less than the value found in the experimental summary Table 4-2. Model simulations were coded to stop for a variety of reasons which made the comparison durations different. Simulations stopped when the model reached the venting pressure for the particular experiment, or at the time the experiment began to vent. In addition, in the case of snow build up on the tanks or loss of a critical region of data the simulations were stopped prior to these events. Due to this, results most likely were not affected by the snow accumulation on the tanks. The comparisons between experimental pressure and model pressure were performed at times before the weight of the snow was evident on the sensors. Also, since the two different thermodynamic approaches had different end results, 
each often had different simulation end times. The model with the shortest time determined the comparison duration and the time in which the data were compared.

In general, the stratified approach had higher accuracy than the homogeneous approach. The homogeneous approach performed better at near full fill levels, but deteriorated quickly as the fill level dropped. Stratification and the associated convection effects were believed to be present in the tank thereby making the homogeneous approach inaccurate at lower fill levels. In addition, the homogeneous approach always over predicted while the stratified model under or over predicted depending on the fill level. No conclusive pattern was evident for the stratified model but as a general rule the stratified model under predicted at fill levels below 50\% and over predicted above 50\% fill. The average absolute pressure rise rate error for the stratified model was $28.3 \%$ while the average absolute final pressure error was $19.9 \%$

Not enough data were present to conclude a difference in predicting capability between the two types of tanks due to the inherent structural difference (pump and no pump) from the model for the tank. However, the internal fluid pump most likely changed the internal convection dynamics of the IFP tank because the pump was large. The pump was contained in an approximately 3/4 meter long stainless steel tube which was inserted at an angle into the tank and was exposed to both the liquid and vapor spaces.

Table 4-5 shows the average heat transfer values calculated during the hold time simulations. Pressure rise comparisons, heat transfer rates, and local ambient conditions are graphed in appendix C.

Table 4-5: Hold Time Tests - Simulated Heat Loads

\begin{tabular}{|c|c|c|c|c|c|}
\hline \multicolumn{6}{|c|}{ Non-IFP Tank } \\
\hline Exp. & $\begin{array}{c}\text { Duration } \\
\text { (days) }\end{array}$ & $\begin{array}{l}\text { Avg. Homog. } \\
(\mathrm{kJ} / \mathrm{sec})\end{array}$ & $\begin{array}{l}\text { Avg. Stratified } \\
(\mathrm{kJ} / \mathrm{sec})\end{array}$ & $\begin{array}{c}\text { Total Homog. } \\
(\mathrm{kJ})\end{array}$ & $\begin{array}{c}\text { Total Stratified } \\
(\mathrm{kJ})\end{array}$ \\
\hline \multicolumn{6}{|l|}{1} \\
\hline 2 & 3.9 & 0.021 & 0.021 & $7.2 \mathrm{E}+03$ & $7.2 \mathrm{E}+03$ \\
\hline 3 & 11.0 & 0.023 & 0.023 & $2.2 \mathrm{E}+04$ & $2.2 \mathrm{E}+04$ \\
\hline 4 & 7.2 & 0.022 & 0.023 & $1.4 \mathrm{E}+04$ & $1.4 \mathrm{E}+04$ \\
\hline 5 & 15.6 & 0.020 & 0.021 & $2.7 \mathrm{E}+04$ & $2.8 \mathrm{E}+04$ \\
\hline 6 & 12.6 & 0.019 & 0.020 & $2.1 \mathrm{E}+04$ & $2.1 \mathrm{E}+04$ \\
\hline 7 & 9.0 & 0.019 & 0.019 & $1.4 \mathrm{E}+04$ & $1.5 \mathrm{E}+04$ \\
\hline 8 & 0.5 & 0.020 & 0.021 & $9.2 \mathrm{E}+02$ & $9.2 \mathrm{E}+02$ \\
\hline 9 & 7.3 & 0.023 & 0.024 & $1.5 \mathrm{E}+04$ & $1.5 \mathrm{E}+04$ \\
\hline \multicolumn{6}{|c|}{ IFP Tank } \\
\hline \multicolumn{6}{|l|}{1} \\
\hline 2 & 11.1 & 0.019 & 0.019 & $1.8 \mathrm{E}+04$ & $1.9 \mathrm{E}+04$ \\
\hline 3 & 6.6 & 0.019 & 0.020 & $1.1 \mathrm{E}+04$ & $1.1 \mathrm{E}+04$ \\
\hline 4 & 4.1 & 0.019 & 0.019 & $6.7 \mathrm{E}+03$ & $6.8 \mathrm{E}+03$ \\
\hline 5 & 7.3 & 0.020 & 0.020 & $1.2 \mathrm{E}+04$ & $1.3 \mathrm{E}+04$ \\
\hline
\end{tabular}


The type of thermodynamic approach used did not greatly change the total average heat transfer rate into the tank. However, the two thermodynamic approaches performed radically differently as the average absolute error for pressure rise rate of homogeneous approach was $118 \%$ while $20 \%$ for the stratified approach. The difference in performance was due to each approach's underlying assumptions. The stratified approach did not treat the vapor and liquid regions as one, well mixed, space as was done in the homogeneous approach. Therefore, dynamics at the vapor-liquid interface, specifically, evaporation and condensation were handled differently. The evaporation rate at the interface was highly depended on the heat transfer to liquid inside the tank for the stratified approach. The two separate regions maintained different temperatures profiles based on the evolution of convection. While for the homogeneous approach all the energy input into both regions attributed to the evaporation rate and therefore pressure rise. This caused the homogeneous model to be accurate at high fill levels where most of the heat transfer was into the liquid while at low fill levels this was not the case. The amount of heat transfer entering the two phases in the tank was directly related by fill level because the heat transfer model distinguished between the wetted areas. Table 4-6 shows the average heat leak rate to the liquid and vapor phases for the stratified thermodynamic approach.

Table 4-6: Hold Time Simulations - Average Heat Leak to Liquid and Vapor Phases

\begin{tabular}{|c|c|c|c|c|}
\hline \multicolumn{5}{|c|}{ Non-IFP Tank } \\
\hline Exp. & Duration (days) & Fill & Avg. Liquid (kJ/sec) & Avg. Vapor (kJ/sec) \\
\hline 1 & & & & 0.0035 \\
\hline 2 & 3.9 & 0.88 & 0.0179 & 0.0116 \\
\hline 3 & 11.0 & 0.52 & 0.0118 & 0.0153 \\
\hline 4 & 7.2 & 0.31 & 0.0076 & 0.0072 \\
\hline 5 & 15.6 & 0.72 & 0.0137 & 0.0106 \\
\hline 6 & 12.6 & 0.45 & 0.0089 & 0.0130 \\
\hline 7 & 9.0 & 0.28 & 0.0062 & 0.0173 \\
\hline 8 & 0.5 & 0.10 & 0.0032 & 0.0098 \\
\hline 9 & 7.3 & 0.65 & 0.0138 & \\
\hline & & & IFP Tank & 0.0092 \\
\hline 1 & & & & 0.0138 \\
\hline 2 & 11.1 & 0.54 & 0.0101 & 0.0153 \\
\hline 3 & 6.6 & 0.25 & 0.0058 & 0.0128 \\
\hline 4 & 4.1 & 0.14 & 0.0039 & \\
\hline 5 & 7.3 & 0.35 & 0.0075 & \\
\hline
\end{tabular}


Some difference in heat transfer between the homogeneous and the stratified thermodynamic approach was evident, especially in long simulations. A temperature distribution eventually formed which changed the heat transfer calculated to each region as the simulation progressed.

Figure 4-6 shows a temperature distribution calculated by the stratified approach for the 4th experiment simulation of the NON-IFP tank.

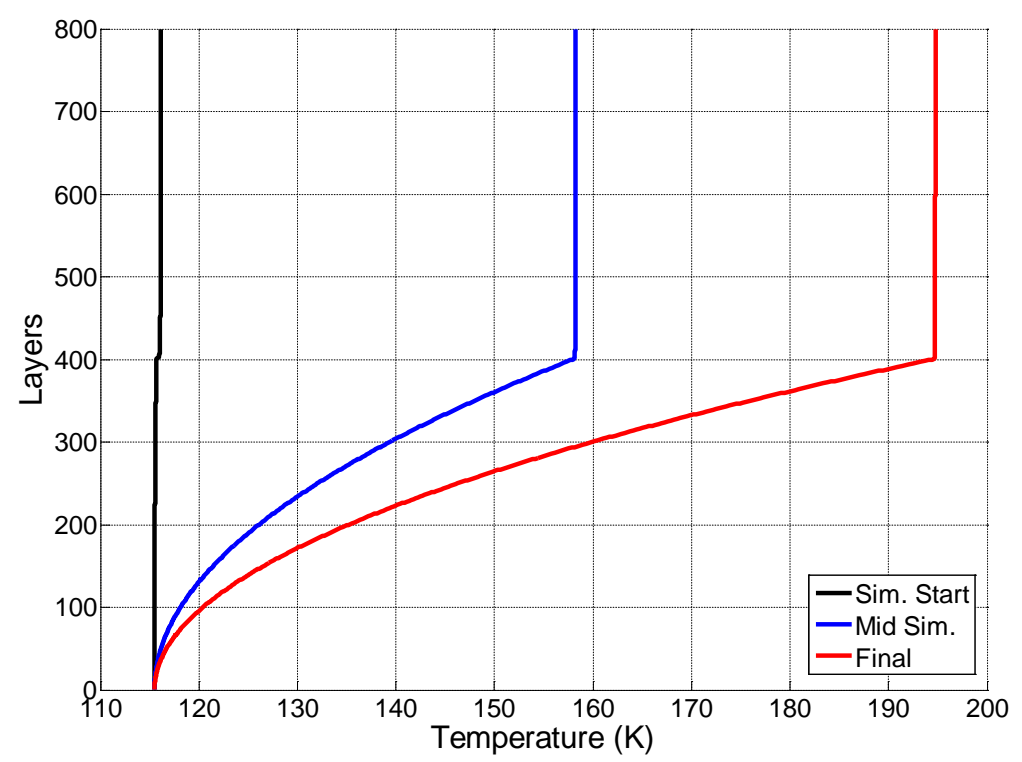

Figure 4-6: Exp. 4 NON-IFP Tank Hold Time Sim. - Tank Heat Distribution

During long simulation periods the heat transfer rate into the vapor phase dropped while heat transfer to the liquid region increased as compared to the homogeneous approach. The case shown in the figure was an extreme example of a temperature distribution variations calculated by the stratified model. The figure shows three lines each indicating the temperature variation of the layers used by the heat transfer model at specific times in the simulation. The first black line was after the first iteration, the blue line at the half way point in time, and the red line was the final temperature distribution. At 400 layers was the vaporliquid interface. The fluid height was not half the length of the tank but at the distance dictated by the fill level. As discussed earlier each phase region was equally divided by the specified number of layers. The fill level at the time was $31 \%$. 


\subsection{NER Experiments}

\subsubsection{Data Collection}

The normal evaporation experiments (NER) were conducted on two different types of LNG vehicle tanks, the same tanks used in the pressure rise experiments. Each tank was tested by opening the pressure release valve and allowing the dewar to vent to atmospheric pressure. The vented gas was directed through a flexible tube to a full flow sampling system (FFS). The FFS consisted of a blower, methane detector, associated piping, and a mass air flow sensor. Air was drawn through the blower and down the length of a pipe where a tap was made to a LGR (Los Gatos Research) Ultraportable Greenhouse Gas Analyzer. The analyzer was then used to find the concentration of methane in the flow. Flow rate and the concentration of methane were used to extrapolate an evaporation rate from the vehicle tank. Error associated with the final evaporation rate was $+/-4.4 \% \mathrm{~g} / \mathrm{hr}$. More information about the measurement device and experimental setup can be found in Appendix B. The mass of methane being released by the tank was multiplied by the heat of vaporization at 1 atmosphere to calculate a heat leak rate into the vehicle tanks. Figure 4-7 shows the heat leak of the first experiment of the NON-IFP tank.

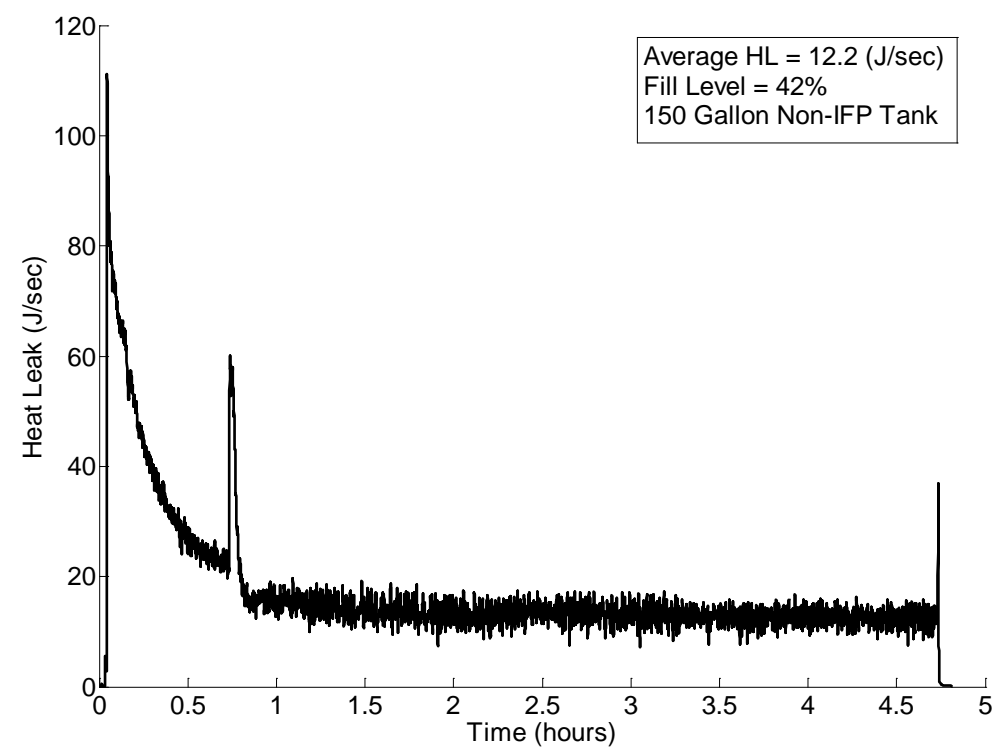

Figure 4-7: NON-IFP Tank NER Test 1 - Heat Leak Extraplated from Normal Evaporation Rate by

\section{Using Heat of Vaporization.}

The average of the steady state evaporation rate was assumed to be the heat transfer into the tank. Each test was performed at a different fill level and a variety of environmental conditions. Continuous data were collected from the same sensors used in the hold time experiments. A summary of the data collected for the NER experiments is presented in Table 4-7. 
Table 4-7: NER Experiment Summary

\begin{tabular}{|c|c|c|c|c|c|c|c|c|}
\hline Trials & Fill & $\begin{array}{c}\text { Btm. } \\
\text { Surface } \\
\text { Temp. }\end{array}$ & $\begin{array}{c}\text { Top } \\
\text { Surface } \\
\text { Temp. }\end{array}$ & $\begin{array}{c}\text { Btm. } \\
\text { Solar } \\
\text { Loading }\end{array}$ & $\begin{array}{c}\text { Top } \\
\text { Solar } \\
\text { Loading }\end{array}$ & $\begin{array}{c}\text { Ambient } \\
\text { Temp. }\end{array}$ & NER & $\begin{array}{c}\text { Heat } \\
\text { Leak }\end{array}$ \\
\hline NON-IFP 1: & 0.42 & 288 & 293 & 7.7 & 76.6 & 289 & 0.024 & 12.2 \\
\hline NON-IFP 2: & 0.70 & 281 & 281 & 7.8 & 165.4 & 279 & 0.036 & 18.3 \\
\hline NON-IFP 3: & 0.30 & 288 & 292 & 8.5 & 131.8 & 285 & 0.021 & 10.7 \\
\hline IFP 1: & 0.26 & 274 & 286 & 0.1 & 18.7 & 275 & 0.017 & 8.68 \\
\hline IFP 2: & 0.37 & 293 & 312 & 25.5 & 521.2 & 294 & 0.032 & 16.4 \\
\hline IFP 3: & 0.16 & 285 & 301 & 1.8 & 149.8 & 288 & 0.009 & 4.45 \\
\hline
\end{tabular}

\subsubsection{NER Comparison}

It was apparent from Li et al. the fill level affected the normal evaporation rate in cryogenic tanks. Lower fill levels correlated to lower evaporation rates. At low fill levels a high temperature distribution formed in the vapor space of the tank [28]. In general the NER experiments followed the same trend, lower evaporation rate with lower fill level, as the data indicates in Table 4-7. No data on the temperature distribution inside the vehicle tanks were collected. Therefore, the heat transfer model assumed a homogeneous temperature distribution when compared to the data. The overall heat transfer rate calculated by the model did not match the heat leak indicated by the normal evaporation rate test. However, considering the vapor was able to escape the tank, heat transfer to the liquid was reasoned to be the dominant controller of the evaporation rate. Therefore, a comparison was made between the calculated heat transfer to the liquid and the measured NER values. First, the temperature, solar loading, and tank dimensions were input to the model to calculate a transient heat leak value. The average of the simulation was used to compare to the measured values. The heat transfer through the surface area wetted by the liquid phase, vapor phase, and total amount was compared to the NER rate.

Table 4-8 shows the results of the simulations comparing the heat transfer through the wetted areas exposed to different phases of the LNG and the measured NER. In the table the vapor, liquid, and total columns referred to the average calculated heat transfer to the vapor region, liquid region, and the sum total. 
Table 4-8: NER Wetted Area Comparion

\begin{tabular}{|l|c|c|c|c|c|}
\hline Trials & Fill & Vapor & Liquid & Total & Measured \\
\hline Tank & (fraction) & $\mathrm{J} / \mathrm{sec}$ & $\mathrm{J} / \mathrm{sec}$ & $\mathrm{J} / \mathrm{sec}$ & $\mathrm{J} / \mathrm{sec}$ \\
\hline NON-IFP 1: & 0.42 & 13.9 & 10.4 & 24.4 & 12.2 \\
\hline NON-IFP 2: & 0.70 & 7.6 & 15.7 & 23.3 & 18.3 \\
\hline NON-IFP 3: & 0.30 & 17.1 & 8.1 & 25.2 & 10.7 \\
\hline IFP 1: & 0.26 & 15.8 & 6.4 & 22.3 & 8.7 \\
\hline IFP 2: & 0.37 & 15.3 & 9.5 & 24.8 & 16.4 \\
\hline IFP 3: & 0.16 & 20.4 & 5 & 25.4 & 4.4 \\
\hline
\end{tabular}

Heat leak through the liquid wetted area as calculated by the model followed the same trend as the measured values. As the fill level lowered the heat transfer to liquid also lowered and was of similar value to the evaporation rate. The average difference between the calculated heat leak to the liquid and the measured evaporation rate heat transfer values was $18.2 \%$. It was likely the evaporation rate in the tank was also effected by the heat transfer to the vapor space and may have accounted for the difference. No thermodynamics involving evaporation at the interface was considered as this was a comparison only with the 1-D heat transfer approach. Error in the individual cases was most likely caused by the lack of temperature distribution data and no coupled liquid-vapor interface modeling as found in the stratified approach.

As seen from the hold time tests the homogeneous thermodynamic approach greatly over predicted pressure rise at low fill levels while being accurate at high fill levels. The results of the NER analysis supported the finding. As the fill level increased the evaporation rate was closer to the total heat transfer calculated by the model. At low fill levels the evaporation rate appeared to be tied closely to the amount of energy reaching the liquid phase. Therefore, the homogeneous approach was likely over estimating the evaporation rate thus the pressure rise. As experience with the stratified approach indicated, and previous work suggests, for a closed cryogenic tank the pressure rise was dictated by internal convection and dynamics at the vapor-liquid interface. 


\subsection{Full Station Tank Model Experiments}

In order to receive data from operational stations a non-disclosure agreement was signed between WVU and the station owners. Certain data can be presented but other information such as the station owner, exact station dimensions, and exact station location cannot be revealed. Therefore, the data acquired from the two stations were referred to as audit 1 station and audit 2 station. The audit 1 station was located in Nevada and the audit 2 station was located in Utah to put weather patterns experienced into perspective. Approximately three weeks of data was collected from each station. Data were split into sections to compare with the model. Segments ran from LNG tanker offload-to-offload or from offload-to-manual venting event. Information about the fleets which visited the stations was also collected. Weather data for the LNG stations was pulled from the National Oceanic and Atmospheric Association (NOAA) weather stations in the vicinity. Typically ambient temperature was only available. The data collected for the audit stations are presented in the next section and a walkthrough of the station behavior is also given.

\subsubsection{Audit 1 Data Collection}

At audit 1 the station had pre-installed data collecting sensors. These sensors provided the data used for the audit comparisons. Data on the tank pressure, tank fill level, dispense pump pressure, dispense pump flow rate, offload pump pressure, and offload pump temperature were provided by the station owners for the audit. Figure 4-8 shows all the pressure data collected at the audit 1 station. The station utilized one 25,000 gallon horizontal tank and two dispensers.

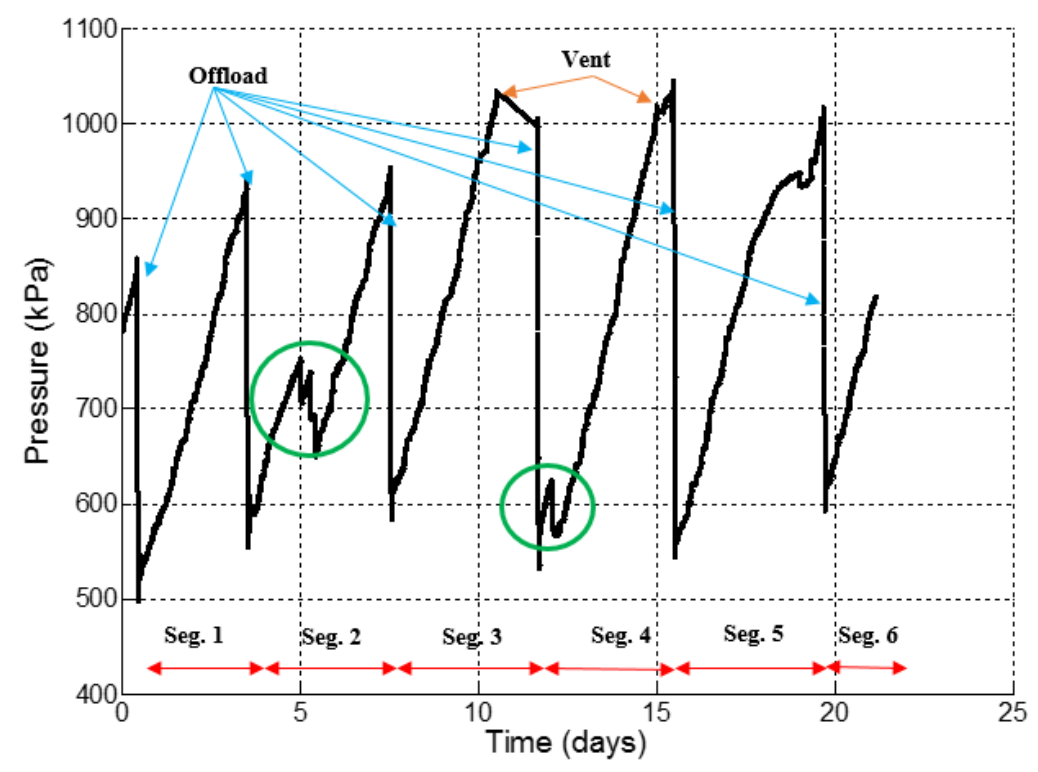

Figure 4-8: Audit 1-Tank Pressure History 
The audit was split into six segments indicated by the red arrows in the figure. The first five were from station tank offload-to-offload. Segment 6 duration was from an offload to the end of the audit. The blue lines on the figure point to the offload events where a tanker arrived to refill the station. Offload events started at the peak pressure and finished at the end of the pressure drop. Almost all the steep decreases in the pressure rise curves in audit 1 were from offload events. The green circled areas represented high pressure rise regions for the station which ended upon a vehicle arriving. Venting occurred twice at the station through the PRV and was due to over pressurization. The orange arrows point to these two venting events. Venting was not a single event but a continuous vent of LNG vapor out of the tank. The PRV vented at approaximately $1032 \mathrm{kPa}$ (135 psig). The pressure sensors used by the station had a qouted accuracy of +/- $1.3 \mathrm{kPa}$ (0.1885 psia).

Figure 4-9 shows the fill level of the station over the entirety of the audit. The fill level was reported in terms of fraction by volume with 1 being full and 0 being empty. The fill level sensor for the station reported data in gallons and inches of water. The nominal volume of the station tank was $94.6 \mathrm{~m}^{3}$ (25,000 gallons), however, the rated warm volume was $101.3 \mathrm{~m}^{3}$ (26,760 gallons) and rated cold level volume was $90.5 \mathrm{~m}^{3}$ (23,904 gallons). The warm volume referred to volume at ambient temperature and the cold volume refered to the volume with liquid nitrogen in the tank which was 77 kelvin and caused the vessel to shrink. The fill level was calculated using the nominal volume of $94.6 \mathrm{~m}^{3}$ (25,000 gallons).

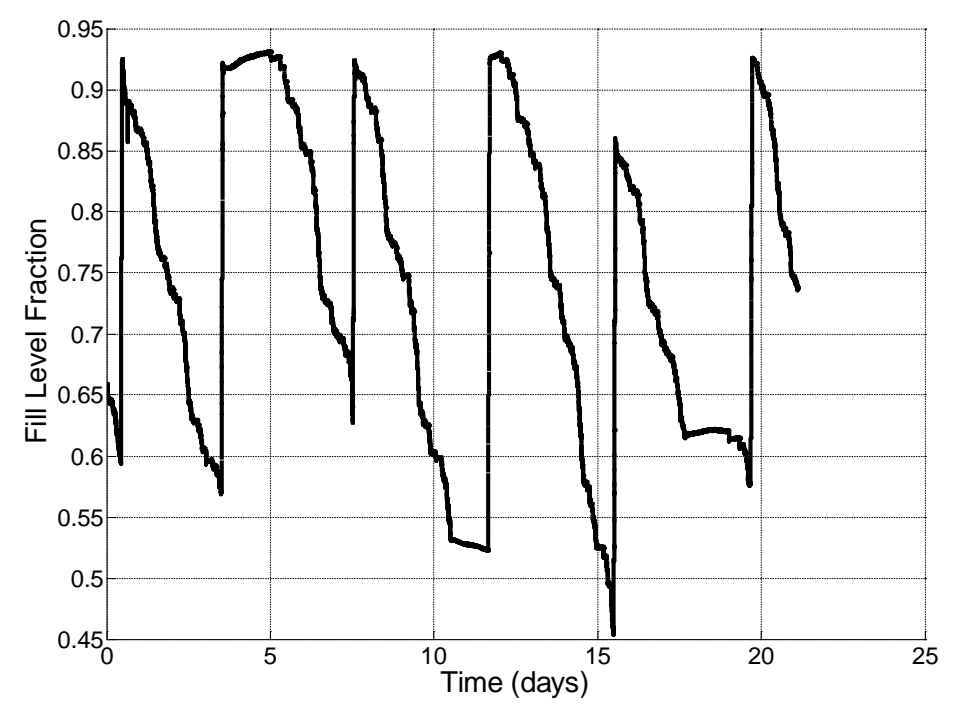

Figure 4-9: Audit 1- Station Fill Level

Figure 4-10 shows the dispense pump pressure. The peaks in the dispense pump pressure were fueling events. Other than the dispensing pressure peaks the dispense pump tracked the pressure of the station almost exactly with only a $7 \mathrm{kPa}$ to $14 \mathrm{kPa}$ difference at any given time. 


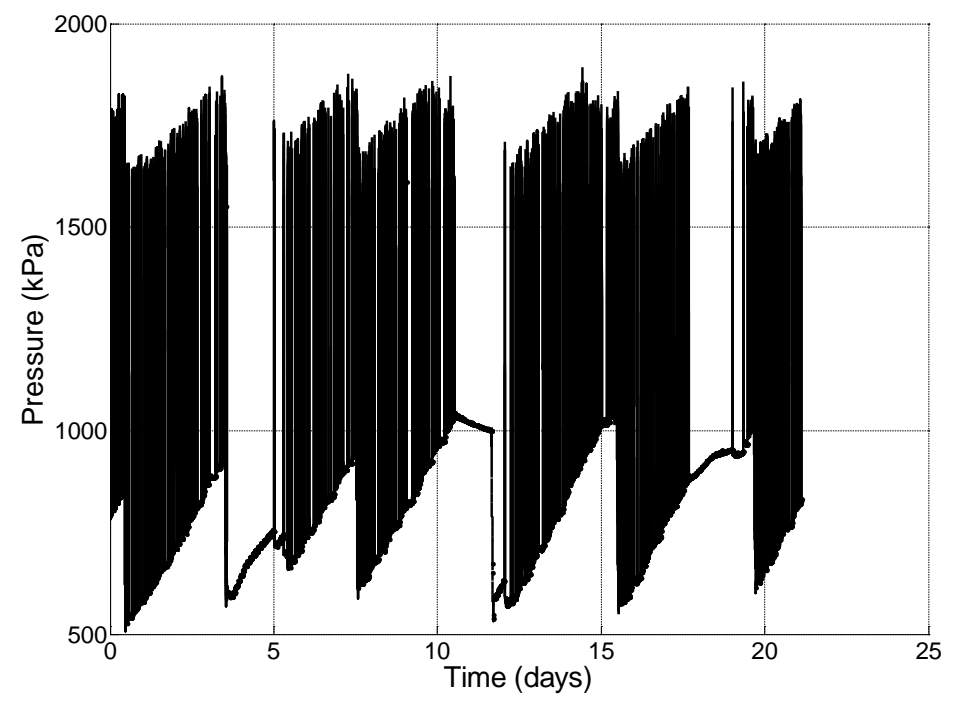

Figure 4-10: Audit 1- Dispense Pressure

The offload pump was used to refuel the station tank when it was low. On average the station tank was refilled at the 56\% fill level. Figure 4-11 shows the temperature and pressure of the offload pump. Pressure in the offload pump tracked the station tank pressure for a time then decayed to atmospheric conditions during each segment. LNG contained within the pump housing and lines was allowed to expand into the station tank through a one way valve as it warmed. After a certain point the pressure in the offload pump decayed to atmospheric pressure. Whether the additional LNG vapor in the lines continued to expand into the tank after the decay point or leak to the atmosphere was uncertain.
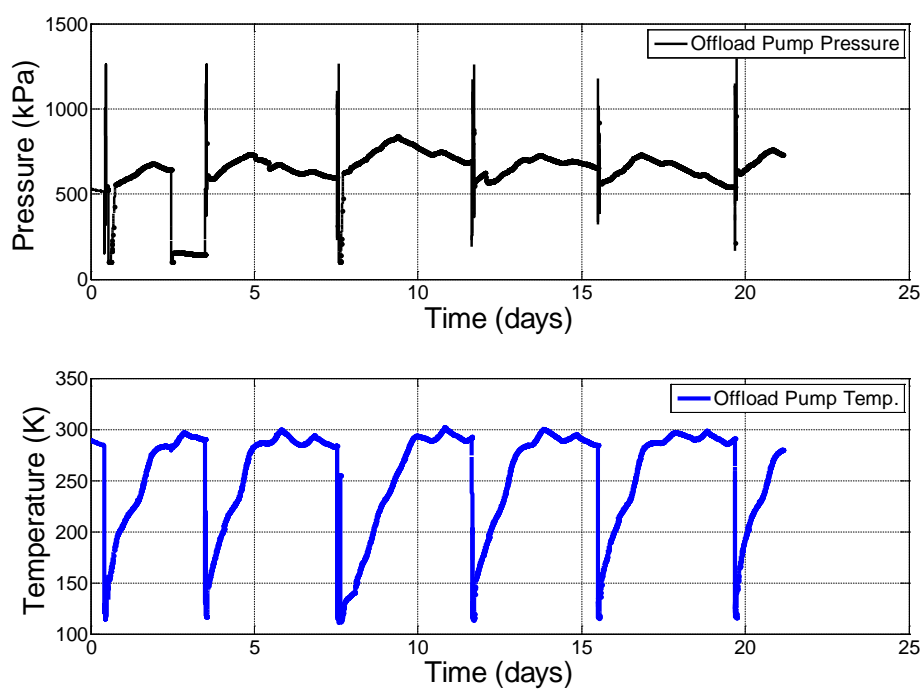

Figure 4-11: Audit 1- Offload Pump Pressure and Temperature 
The green circled regions in Figure 4-8 showed high pressure rise rates until vehicles arrived at the station. It was possible the increased pressure rise rate was caused by the addition of warm LNG vapor being added by the offload pump lines. This was known as a false pressure head, as the pressure in the tank did not reflect its actual bulk state. Once vehicles arrived at the station this process was disturbed and the station pressure quickly returned to normal.

Audit station 1 had 768 vehicle fueling transactions during the course of the audit. A transaction list was provided by the station owners. The list detailed the amount of DGEs (diesel gas equivalent) dispensed to the vehicles along with time. Some vehicles refueled more than once causing the transaction $\log$ to list the same vehicle multiple times. After the data were reduced this translated to 684 vehicles visiting the station. Of the 684 vehicles 374 were witnessed and additional data were recorded. Data collected from the observed vehicles included arrival tank pressure, arrival tank fill level, vented pressure, and refueled pressure. Table 4-9 shows a summary of the transaction information for audit 1.

Table 4-9: Audit 1 Vehicle Transaction Summary

\begin{tabular}{|l|r|l|}
\hline Audit Duration & 21 & Days \\
\hline Minimum Dispensed & 2 & DGE \\
\hline Maximum Dispensed & 141 & DGE \\
\hline Average DGE/Vehicle & 44 & DGE \\
\hline Total Dispensed & 34,505 & DGE \\
\hline Daily Average & 1,568 & DGE \\
\hline Total Transactions & 768 & - \\
\hline Average Transactions & 37 & $\# /$ day \\
\hline
\end{tabular}

Table 4-10 gives a summary of the observed additional vehicle data at the audit 1 station. For the vehicles where the additional data were not available the averages were used by the model. Almost all of the vehicles observed carried two LNG tanks, one a 120 gallon tank and the other an 80 gallon tank with five observed exceptions. Of the vehicles observed $76 \%$ vented back to the station. If a vehicle arrived at the station above approximately $930 \mathrm{kPa}$ (135 psig) the vehicle vented LNG vapor back to the station to reduce its pressure prior to refueling. The average fueling time for a vehicle was approximately 12 minutes.

Table 4-10: Audit 1 Fleet Tank Data Summary

\begin{tabular}{|l|l|l|l|}
\hline Name & Value & Standard Deviation & Units \\
\hline Average Arriving Pressure & 1171 & 265 & $\mathrm{kPa}$ \\
\hline Average Arriving Fill Level & 0.383 & 0.21 & fraction \\
\hline Average Vented Pressure & 895 & 148 & $\mathrm{kPa}$ \\
\hline Average Refueled Pressure & 1135 & 161 & $\mathrm{kPa}$ \\
\hline
\end{tabular}


A summary of data collected for each of the segments in audit 1 is shown in Table 4-11. Segments 3 and 4 of the first audit vented through the PRV valve. Segment 3 had a long continuous vent with no vehicle traffic while segment 4 vented during vehicle traffic. Segment 6 of the audit 1 ends at the end of the audit and not an offload event as the others do.

Table 4-11: Audit 1 Experimental Data Summary by Segment

\begin{tabular}{|r|r|r|r|r|r|r|r|}
\hline Seg. & $\begin{array}{c}\text { Duration } \\
\text { (hrs.) }\end{array}$ & $\begin{array}{c}\text { Vent Time } \\
\text { (hrs.) }\end{array}$ & $\begin{array}{c}\text { Avg. } \\
\text { Temp } \\
(\mathrm{K})\end{array}$ & Vehicles & $\begin{array}{c}\text { Fuel Dispensed } \\
\text { (kg) }\end{array}$ & $\begin{array}{c}\text { Initial Press. } \\
(\mathrm{kPa})\end{array}$ & $\begin{array}{c}\text { Peak Press. } \\
(\mathrm{kPa})\end{array}$ \\
\hline 1 & 69.4 & & 290 & 105 & 12896 & 533 & 936 \\
\hline 2 & 92.6 & & 291 & 87 & 10510 & 583 & 949 \\
\hline 3 & 96.9 & 69.6 & 291 & 124 & 14895 & 609 & 1028 \\
\hline 4 & 90.1 & 77.5 & 292 & 147 & 17215 & 575 & 1042 \\
\hline 5 & 98.7 & & 291 & 90 & 10944 & 561 & 1012 \\
\hline 6 & 32.4 & & 288 & 59 & 7841 & 622 & 812 \\
\hline
\end{tabular}

\subsubsection{Audit 2 Data Collection}

At the second audit station only pressure rise data were collected continuously while fill level values where collected for specific dates and times for the station tank. Data were also collected on the vehicle fleet which arrived at the station during audit 2. Figure 4-12 shows the pressure behavior of the station. The station used a vertical 15,000 gallon storage tank, and had two dispensers.

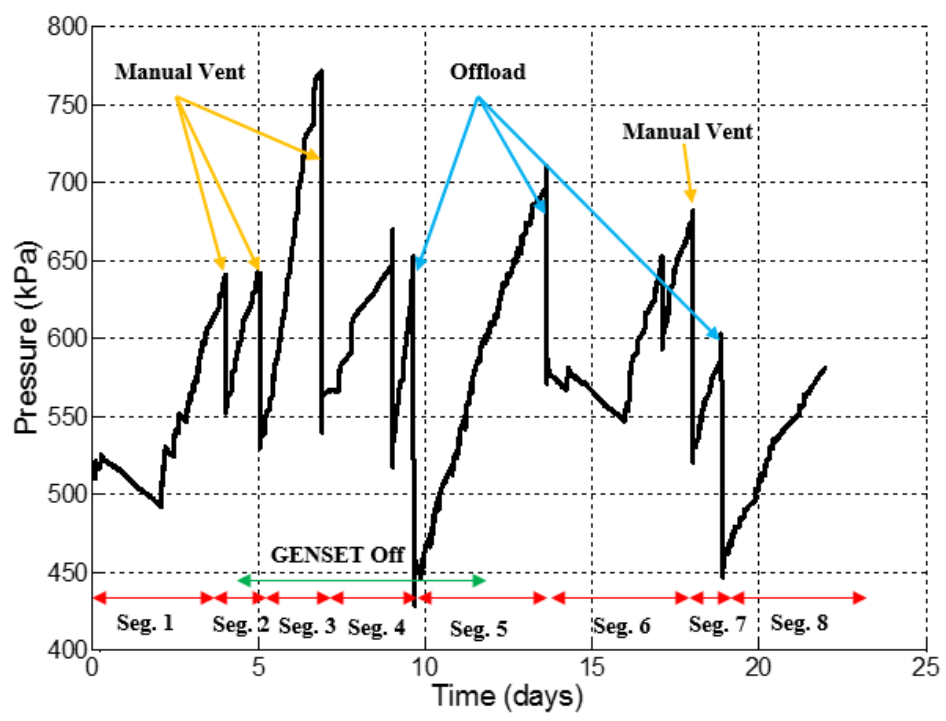

Figure 4-12: Audit 2 - Tank Pressure History

Audit station 2 differed from the first audit station in a number of ways. First, the station used a pressure mitigation strategy due to the relatively low number of vehicles visiting it. A generator which 
removed LNG vapor out of the station tank to provide power and supply electricity back to the grid was connected to the station tank to mitigate pressure rise. The generator had a rated power of $30 \mathrm{~kW}$, efficiency of $27 \%$, and pulled $8.7 \mathrm{~kg} / \mathrm{hr}$ of methane from the station tank at peak power. The mass removed by the generator during operation was considered constant at $8.7 \mathrm{~kg} / \mathrm{hr}$ in the model. The audit 2 station also had a different heat management strategy than the first station. The piping of the station was allowed to warm in the interim between vehicle refueling. This aided the pressure rise of the station over long periods where no vehicles refueled. Otherwise the station behaved similarly to the audit 1 station.

For comparison of the model to the data, eight segments were created. The red lines indicate the segments in Figure 4-12. The generator was on for only a few of the segments. During segments 3 and 4 the generator was off the entire time while for segments 2 and 5 the generator was on for a portion of the time. Pressure dropped in the station during portions of segment 1 and 6 when no vehicles were arriving at the station and the generator was on. During the course of the audit the tank was refilled three times as shown in Table 4-12.

Table 4-12: Audit 2 Station Offloads

\begin{tabular}{|c|c|c|c|c|}
\hline \multirow{2}{*}{ Segment } & \multirow{2}{*}{ Start time } & \multirow{2}{*}{ End time } & \multicolumn{2}{|c|}{ Station [kPa | liter] } \\
\cline { 4 - 5 } & & & Before & After \\
\hline 4 & $2: 25 \mathrm{PM}$ & $4: 23 \mathrm{PM}$ & $638 \mid 19071$ & $435 \mid 49055$ \\
\hline 5 & $2: 00 \mathrm{PM}$ & $4: 00 \mathrm{PM}$ & $695.7 \mid 31112$ & $582 \mid 43082$ \\
\hline 7 & $8: 10 \mathrm{PM}$ & $10: 00 \mathrm{PM}$ & $585 \mid 27168$ & $460 \mid 49097$ \\
\hline
\end{tabular}

The station tank was also manually vented numerous times. Table 4-13 shows the manual venting witnessed during the audit. The manual vents were conducted to keep the station pressure below $793 \mathrm{kPa}$ (115 psia) which was advantageous for the vehicle fleet. Vehicles with internal fluid pumps were the primary vehicles visiting the station. These vehicles can receive colder LNG than vehicles not utilizing an internal tank pump. The lower the station pressure, the lower the LNG temperature, and the higher density. The higher density fuel means the vehicles were able to travel farther on a tank providing incentive for the station to remain as cold as possible.

Table 4-13: Audit 2 Manual Station Vent (Witnessed)

\begin{tabular}{|c|c|c|c|}
\hline Segment & Time & Before $[\mathrm{kPa} \mid$ liter $]$ & After $[\mathrm{kPa} \mid$ liter $]$ \\
\hline 1 & $1: 00 \mathrm{AM}$ & $639 \mid 37627$ & $556 \mid 36908$ \\
\hline 2 & $1: 00 \mathrm{AM}$ & $646 \mid 31684$ & $529 \mid 31116$ \\
\hline 3 & $9: 30 \mathrm{PM}$ & $772 \mid 23625$ & $547 \mid 22712$ \\
\hline 6 & $1: 00 \mathrm{AM}$ & $681 \mid 31763$ & $529 \mid 30696$ \\
\hline
\end{tabular}


While Table 4-13 shows the witnessed manual vents, two other large pressure drops were present in segments 4 and 7. These most likely were manual venting events also. Using the data available, overall at the station approximately 3267 liters (863 gallons) of LNG was released to the atmosphere. More about emissions from the second audit station is discussed in the emission comparison section later in the chapter.

The second audit station had employees on hand who filled the vehicle tanks and collected information about each vehicle. Therefore, thorough data were provided by the station operators for the vehicles served. Table 4-14 shows a summary of the vehicle transactions at the station.

Table 4-14: Audit 2 Vehicle Transaction Summary

\begin{tabular}{|l|r|c|}
\hline Audit Duration & 22 & Days \\
\hline Minimum Dispensed & 9.8 & DGE \\
\hline Maximum Dispensed & 88.7 & DGE \\
\hline Average DGE/Vehicle & 34.6 & DGE \\
\hline Total Dispensed & 14720 & DGE \\
\hline Daily Average & 669.1 & DGE \\
\hline Total Transactions & 426 & - \\
\hline Average Transactions & 19.4 & $\# /$ day \\
\hline
\end{tabular}

Table 4-15 shows the averages for the vehicle pressures during the refilling process. Every vehicle which arrived above $1000 \mathrm{kPa}$ (130 psig) was vented back to the station until the vehicle tank pressure was 1000 $\mathrm{kPa}$ (130 psig). Of the 426 vehicles, 222 or $52 \%$, needed to vent back to the station prior to refueling. Of the vehicles which arrived only six utilized a NON-IFP tank, and none of these tanks needed to vent. The rest utilized IFP tanks.

Table 4-15: Audit 2 Fleet Tank Data Summary

\begin{tabular}{|l|l|l|l|}
\hline Name & Value & Standard Deviation & Units \\
\hline Average Arriving Pressure & 1096 & 229 & $\mathrm{kPa}$ \\
\hline Average Vented Pressure & 998 & 0 & $\mathrm{kPa}$ \\
\hline Average Refueled Pressure & 1103 & 89 & $\mathrm{kPa}$ \\
\hline
\end{tabular}

A summary of data collected for each of the segments in audit 1 and audit 2 is shown in Table 4-16. Over the course of audit 2, four segments ended when a manual vent occurred, 3 ended at an offload, and segments 2 and 7 had possible manual venting events occur in the segment duration. 
Table 4-16: Audit 2 Segment Summary

\begin{tabular}{|r|r|r|r|r|r|r|}
\hline Seg. & $\begin{array}{c}\text { Duration } \\
\text { (hrs.) }\end{array}$ & \multicolumn{1}{c|}{$\begin{array}{c}\text { Avg. Temp } \\
(\mathrm{K})\end{array}$} & Vehicles & $\begin{array}{c}\text { Fuel Dispensed } \\
(\mathrm{kg})\end{array}$ & \multicolumn{1}{c|}{$\begin{array}{c}\text { Initial Press. } \\
(\mathrm{kPa})\end{array}$} & $\begin{array}{c}\text { Peak Press. } \\
(\mathrm{kPa})\end{array}$ \\
\hline 1 & 96.6 & 296 & 74 & 4054 & 505 & 626 \\
\hline 2 & 22.9 & 295 & 28 & 1737 & 549 & 629 \\
\hline 3 & 42.6 & 299 & 44 & 2759 & 523 & 757 \\
\hline 4 & 64.7 & 291 & 20 & 1140 & 550 & 656 \\
\hline 5 & 91.8 & 287 & 109 & 7510 & 435 & 696 \\
\hline 6 & 102.1 & 297 & 65 & 3782 & 562 & 667 \\
\hline 7 & 19.3 & 300 & 18 & 1480 & 516 & 589 \\
\hline 8 & 71.9 & 294 & 45 & 3030 & 447 & 567 \\
\hline
\end{tabular}

\subsubsection{Model Comparison}

The full station model was compared to the audited stations through pressure rise rate. Table 4-17 shows the results of the model comparison. As with the pressure rise experiments both thermodynamic approaches were tested. Appendix $\mathrm{C}$ shows the full pressure rise trend comparisons between the experimental data, and both thermodynamic approaches.

Table 4-17: Audit Pressure Rise Rate and Model Comparison

\begin{tabular}{|c|c|c|c|c|c|c|c|}
\hline \multirow[b]{2}{*}{ Seg. } & \multirow{2}{*}{$\begin{array}{l}\text { Duration } \\
\text { (hrs.) }\end{array}$} & \multirow{2}{*}{$\begin{array}{l}\text { Initial Pressure } \\
\quad(\mathrm{kPa})\end{array}$} & \multicolumn{3}{|c|}{ Final Pressure $(\mathrm{kPa})$} & \multicolumn{2}{|c|}{ Error (\%) } \\
\hline & & & $\begin{array}{l}\text { Exp. } \\
\text { Press. }\end{array}$ & $\begin{array}{l}\text { Homog. } \\
\text { Press. }\end{array}$ & $\begin{array}{l}\text { Stratified } \\
\text { Press. }\end{array}$ & Homog. & Stratified \\
\hline \multicolumn{8}{|c|}{ Audit 1} \\
\hline 1 & 68.0 & 533 & 919 & 869 & 898 & $-13 \%$ & $-5 \%$ \\
\hline 2 & 92.5 & 583 & 938 & 891 & 970 & $-13 \%$ & $9 \%$ \\
\hline 3 & 69.6 & 608 & 1025 & 988 & 1011 & $-9 \%$ & $-3 \%$ \\
\hline 4 & 75.0 & 575 & 976 & 967 & 1026 & $-2 \%$ & $12 \%$ \\
\hline 5 & 97.7 & 561 & 999 & 967 & 971 & $-7 \%$ & $-6 \%$ \\
\hline 6 & 32.0 & 621 & 809 & 779 & 793 & $-16 \%$ & $-9 \%$ \\
\hline \multicolumn{8}{|c|}{ Audit 2} \\
\hline 1 & 96.0 & 497 & 625 & 603 & 763 & $-17 \%$ & $108 \%$ \\
\hline 2 & 22.7 & 549 & 626 & 613 & 630 & $-17 \%$ & $5 \%$ \\
\hline 3 & 40.7 & 523 & 754 & 675 & 669 & $-34 \%$ & $-37 \%$ \\
\hline 4 & 41.2 & 550 & 622 & 656 & 638 & $47 \%$ & $22 \%$ \\
\hline 5 & 90.0 & 436 & 680 & 637 & 758 & $-18 \%$ & $32 \%$ \\
\hline 6 & 47.7 & 562 & 537 & 543 & 638 & $-24 \%$ & $404 \%$ \\
\hline 7 & 19.2 & 516 & 571 & 583 & 580 & $22 \%$ & $16 \%$ \\
\hline 8 & 67.2 & 447 & 561 & 538 & 607 & $-20 \%$ & $40 \%$ \\
\hline
\end{tabular}


The pressure rise rate was compared between experiment and model before the onset of venting. If any venting occurred, from the model or in the experimental data, the point before the venting occurred was used. In two cases, audit 2 segment 4 and segment 6, a large pressure decrease (probable manual venting event) was present mid-simulation. For this reason the durations of the two simulations were short and the pressure comparison not entirely indicative of the behavior of the models. Figure 4-14 show the pressure rise trend comparison during these sections. In the first figure the homogeneous approach reached the vent pressure prior to the actual time; this was the time the models were compared. In Figure 4-14 stratified approach over predicts pressure rise by a large margin while the homogenous approach handles the vapor withdrawal from the generator well.

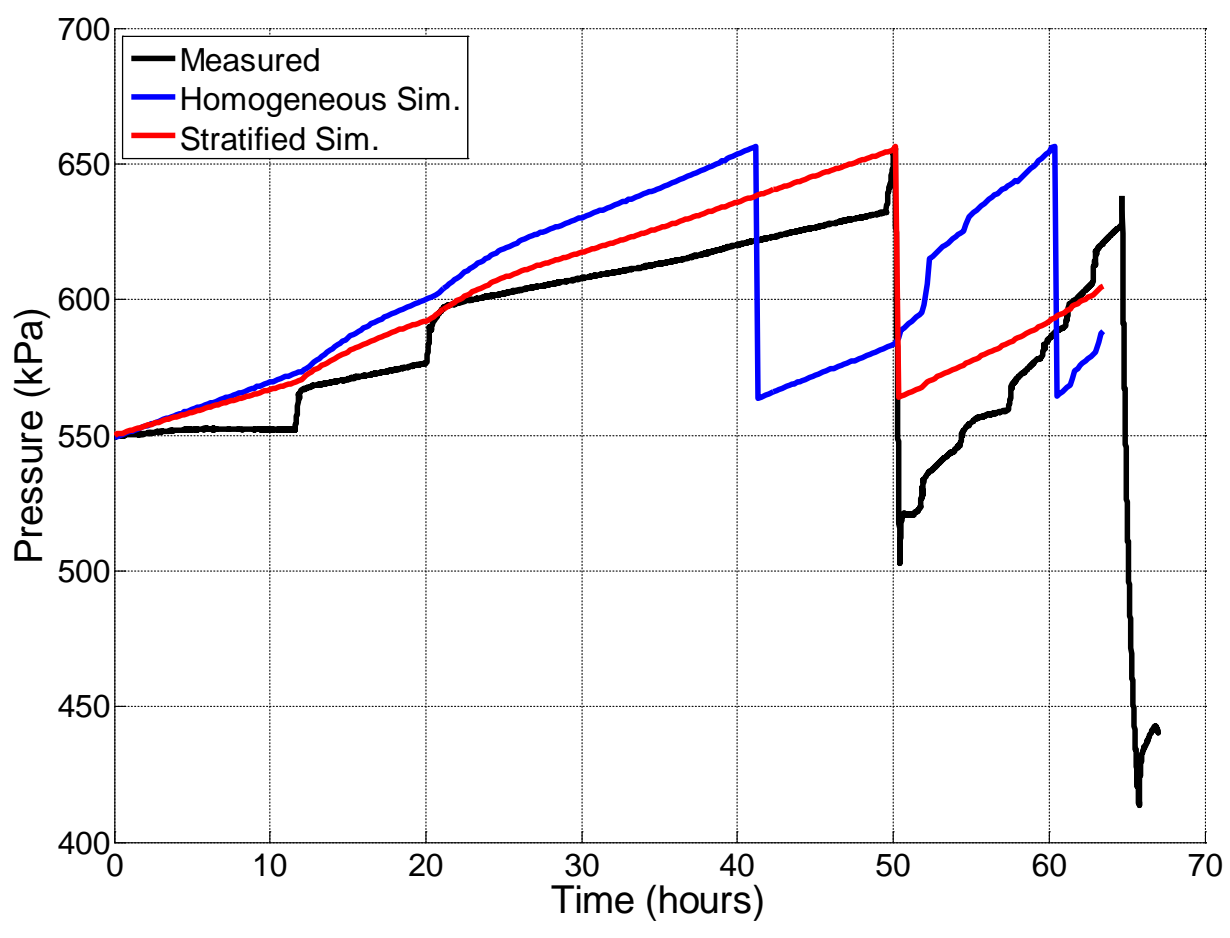

Figure 4-13: Audit 2 Segment 4 Simulation Pressure Comparison 


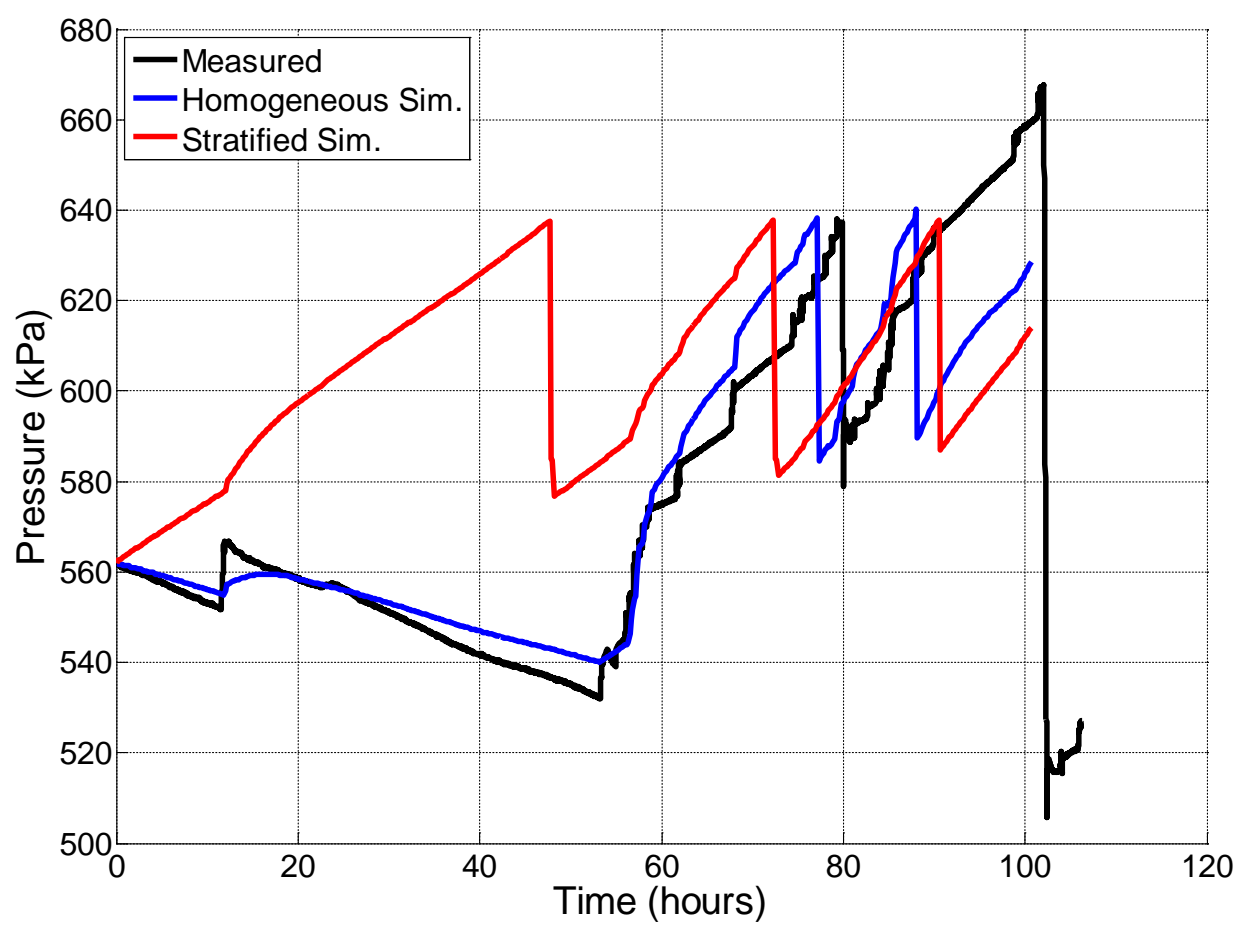

Figure 4-14: Audit 2 Segment 6 Simulation Pressure Comparison

The stratified thermodynamic approach yielded better agreement between the experimental and model results for the first audit but was similar in accuracy for the audit 2 . The average absolute error of the homogeneous model was $10.1 \%$ for audit 1 while the error was $7.5 \%$ for the stratified approach. For the second audit simulations the average absolute percent error was $24.9 \%$ and $25.4 \%$ for the homogeneous and stratified thermodynamic approaches, respectively. Segment 1 and segment 6 for the second audit station both occurred during a time when no vehicles visited the station and the generator was on. The two segments had long periods of negative pressure change. The large percent errors in these two segments were due to a weakness in the stratified model's estimation of the temperature profile change due to the withdrawn vapor. The values from the two segments were not used in the average absolute error calculation. The homogeneous assumption handled these segments consistently.

Pressure rise rates for the experimental data and two thermodynamic approaches are shown in Figure 4-15 and Figure 4-16 for audit 1 and audit 2 respectively. 


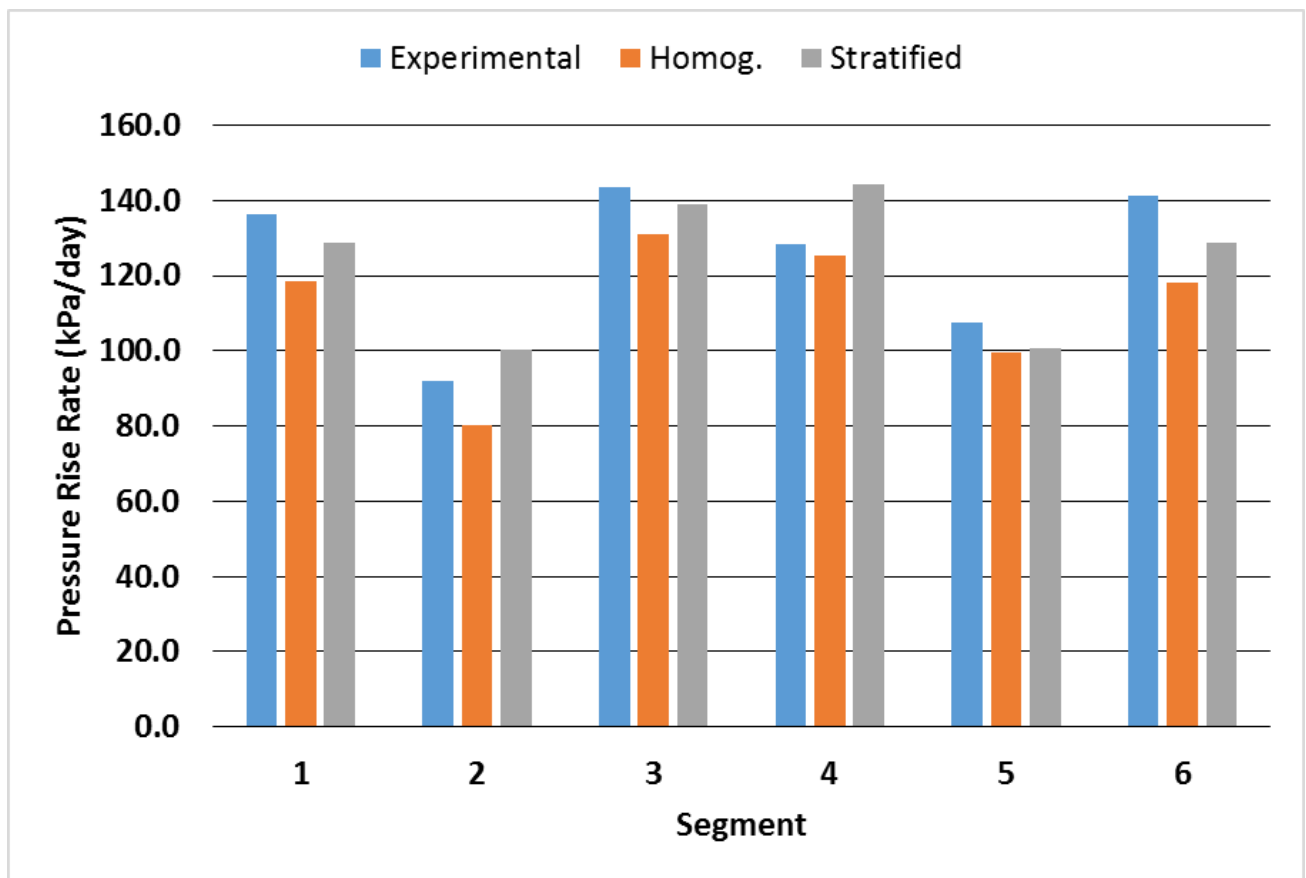

Figure 4-15: Audit 1 Pressure Rise Rate Comparison

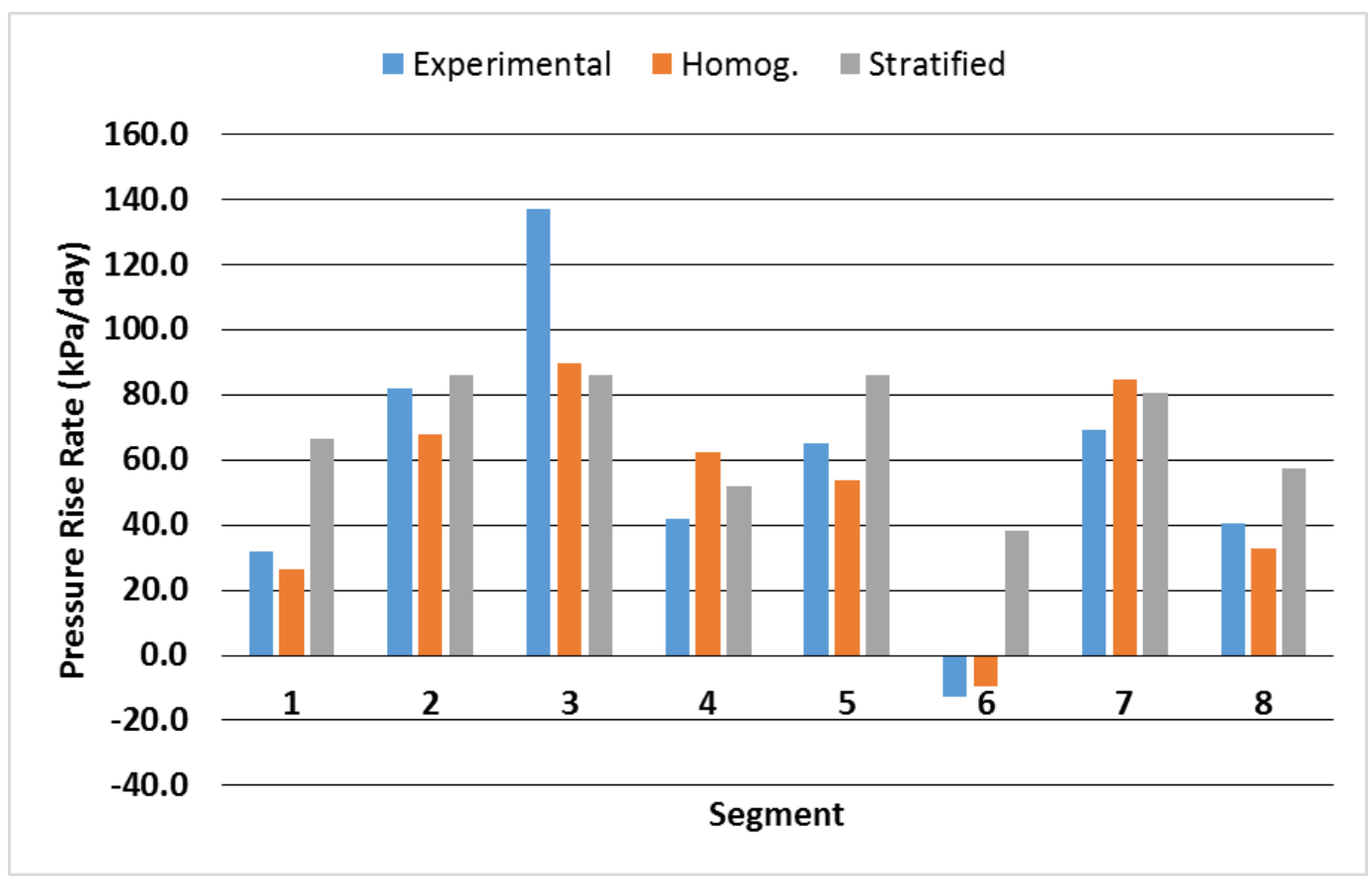

Figure 4-16: Audit 2 Pressure Rise Rate Comparison

The difference in average error between the two audits was caused by a few major reasons. At audit station 1 the owners provided exact dimensions for the piping in the station including type, length, and 
diameter. For the second audit station only estimates of the station piping, collected during the course of the audit were available. The greater inaccuracies in audit 2 were likely due to the inaccurate piping dimensions available. In addition, the second audit station did not keep its piping cooled at all times. It was witnessed at the audit 2 station that a vehicle needed to call in a half an hour ahead of time for the station to circulate LNG throughout the dispense lines prior to the vehicle arriving, in some cases. This behavior occurred usually when the station had seen no vehicles for approximately 12 hours. During this time period less heat transfer would accrue than predicted since it was an assumption in the model that the lines remained cooled.

Underlying the pressure rise trends were the calculated heat loads from the various sections of the model. Each simulation behaved differently in terms of the heat load based on the conditions at the time. Table 4-18 shows the average heat load rates for each segment of the two audits and the total heat transfer rate. These results are shown graphically in Figure 4-17 and Figure 4-18.

Table 4-18: Audit Average Heat Loads

\begin{tabular}{|c|c|c|c|c|c|c|c|}
\hline \multicolumn{8}{|c|}{ Audit 1} \\
\hline \multirow{2}{*}{ Seg. } & \multicolumn{2}{|c|}{ Tank (kJ/sec) } & \multirow{2}{*}{$\begin{array}{l}\text { Vapor Return } \\
(\mathrm{kJ} / \mathrm{sec})\end{array}$} & \multirow{2}{*}{$\begin{array}{c}\text { Dispense } \\
\text { Lines (kJ/sec) }\end{array}$} & \multirow{2}{*}{$\begin{array}{c}\text { Dispenser } \\
(\mathrm{kJ} / \mathrm{sec})\end{array}$} & \multirow{2}{*}{$\begin{array}{c}\text { Offload } \\
\text { Lines (kJ/sec) }\end{array}$} & \multirow{2}{*}{$\begin{array}{c}\text { Total } \\
(\mathrm{kJ} / \mathrm{sec})\end{array}$} \\
\hline & Homog. & Stratified & & & & & \\
\hline 1 & 0.32 & 0.32 & 0.48 & 0.99 & 1.58 & 0.09 & 3.47 \\
\hline 2 & 0.32 & 0.33 & 0.32 & 1.00 & 0.87 & 0.07 & 2.58 \\
\hline 3 & 0.32 & 0.32 & 0.59 & 0.97 & 1.50 & 0.08 & 3.48 \\
\hline 4 & 0.33 & 0.33 & 0.63 & 0.99 & 1.39 & 0.07 & 3.42 \\
\hline 5 & 0.32 & 0.32 & 0.33 & 0.98 & 1.01 & 0.06 & 2.70 \\
\hline 6 & 0.32 & 0.32 & 0.61 & 0.98 & 1.49 & 0.40 & 3.79 \\
\hline \multicolumn{8}{|c|}{ Audit 2} \\
\hline 1 & 0.21 & 0.20 & 0.22 & 0.59 & 0.34 & 0.00 & 1.35 \\
\hline 2 & 0.20 & 0.20 & 0.34 & 0.58 & 0.65 & 0.00 & 1.77 \\
\hline 3 & 0.21 & 0.21 & 0.18 & 0.59 & 0.57 & 0.00 & 1.54 \\
\hline 4 & 0.19 & 0.19 & 0.00 & 0.55 & 0.16 & 0.00 & 0.90 \\
\hline 5 & 0.19 & 0.19 & 0.23 & 0.54 & 0.63 & 0.00 & 1.59 \\
\hline 6 & 0.21 & 0.21 & 0.01 & 0.59 & 0.08 & 0.00 & 0.88 \\
\hline 7 & 0.21 & 0.21 & 0.12 & 0.60 & 0.63 & 0.00 & 1.57 \\
\hline 8 & 0.21 & 0.20 & 0.11 & 0.58 & 0.41 & 0.00 & 1.31 \\
\hline
\end{tabular}




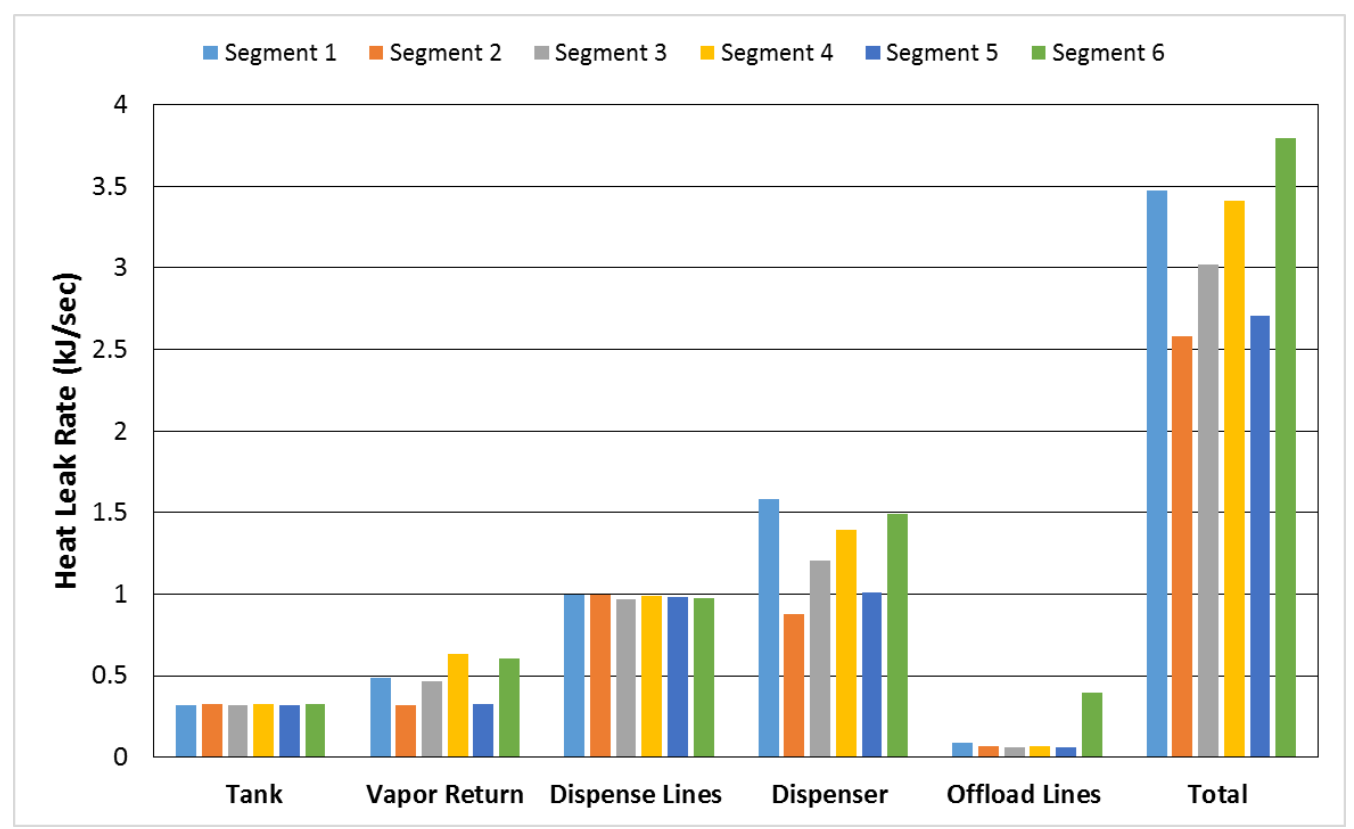

Figure 4-17: Audit 1 Average Energy Transfer Rate for Each Segment by Source

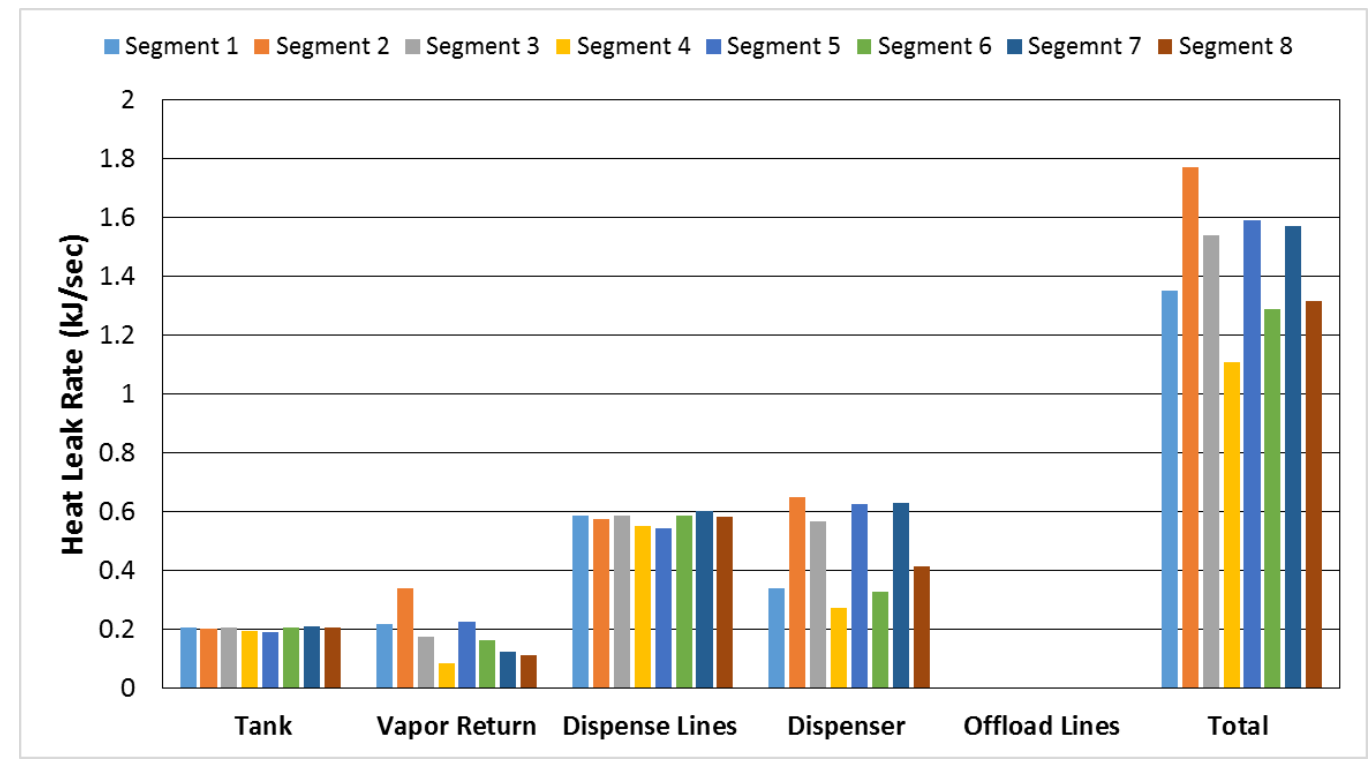

Figure 4-18: Audit 2 Average Energy Transfer Rate for Each Segment by Source

As shown in the table the heat transfer rate through the station tank using the two different thermodynamic models was nearly equal just as seen in the hold time tests. The vapor return heat load depended on the number of vehicles venting back to the station. In the audit 1 simulations all vehicles where before venting and after venting pressure data was unavailable the average values from the measured vehicles were used to calculate venting back to the LNG station. The vapor return values for the first audit 
station were likely to overestimate the actually heat transfer accrued from the vapor return line. This was not the case for the second audit as a complete data set for the vehicles existed. The dispense line heat load was near constant for each audit station and only varied with local ambient temperature. The offload line heat loads were only calculated for audit station 1 because dimensions and behavior data were available. Heat load from the offload line was not calculated for the second audit station due to a lack of information. From the modelling perspective it did not significantly impact the results because the offload piping was a minor heat source. Dispenser heat load to the station was affected by the number of vehicles and the arrival times of the vehicles. When vehicles were fueled in groups and not spread evenly over time the heat load rate tended to be reduced. Table 4-19 shows the fleet behavior and average model calculated dispenser temperature for each of the simulations. The lower the average dispenser temperature the more spread out the vehicles were in the simulation resulting in an increased heat load on the station.

Table 4-19: Audit Simulation - Vehicle and Dispenser Summary

\begin{tabular}{|c|c|c|c|c|}
\hline \multicolumn{5}{|c|}{ Audit 1} \\
\hline Seg. & Duration (days) & \# Vehicles & Vehicles Per Day & Avg. Dispenser Temp. (K) \\
\hline 1 & 2.83 & 105 & 37 & 165 \\
\hline 2 & 3.85 & 87 & 23 & 219 \\
\hline 3 & 3.94 & 124 & 31 & 171 \\
\hline 4 & 3.74 & 147 & 39 & 182 \\
\hline 5 & 4.07 & 90 & 22 & 209 \\
\hline 6 & 1.33 & 59 & 44 & 167 \\
\hline \multicolumn{5}{|c|}{ Audit 2} \\
\hline 1 & 4.00 & 74 & 19 & 242 \\
\hline 2 & 0.94 & 28 & 30 & 194 \\
\hline 3 & 1.69 & 44 & 26 & 205 \\
\hline 4 & 1.74 & 20 & 12 & 264 \\
\hline 5 & 3.75 & 109 & 29 & 183 \\
\hline 6 & 1.99 & 65 & 33 & 285 \\
\hline 7 & 0.80 & 18 & 23 & 197 \\
\hline 8 & 2.80 & 45 & 16 & 227 \\
\hline
\end{tabular}

Table 4-20 shows the total thermal energy from each module. The total column was total heat which reached the LNG stored in the station tank from each module. Dispenser heat load was the greatest contributor in the first audit station while it was second to the dispense piping heat load for the second audit station. Vapor return was likewise greater than the heat load from the station tank in audit 1 but less than the calculated heat load for audit 2. The offload piping was comparatively a low source of heat load into the station. Figure 4-19 and Figure 4-20 show the information found in the tables graphically. 
Table 4-20: Audit Total Heat Transfer

\begin{tabular}{|c|c|c|c|c|c|c|c|}
\hline \multicolumn{8}{|c|}{ Audit 1} \\
\hline Seg. & $\begin{array}{c}\text { Duration } \\
\text { (days) }\end{array}$ & Tank (MJ) & $\begin{array}{c}\text { Vapor } \\
\text { Return (MJ) }\end{array}$ & $\begin{array}{c}\text { Dispense } \\
\text { Lines (MJ) }\end{array}$ & $\begin{array}{c}\text { Dispenser } \\
(\mathrm{MJ})\end{array}$ & $\begin{array}{c}\text { Offload } \\
\text { Lines (MJ) }\end{array}$ & Total (MJ) \\
\hline 1 & 2.83 & 79 & 119 & 244 & 388 & 22 & 852 \\
\hline 2 & 3.85 & 108 & 106 & 332 & 292 & 23 & 862 \\
\hline 3 & 3.94 & 109 & 159 & 329 & 411 & 21 & 1030 \\
\hline 4 & 3.74 & 105 & 205 & 320 & 451 & 23 & 1100 \\
\hline 5 & 4.07 & 113 & 115 & 345 & 356 & 23 & 953 \\
\hline 6 & 1.33 & 37 & 70 & 113 & 173 & 46 & 439 \\
\hline \multicolumn{8}{|c|}{ Audit 2} \\
\hline 1 & 4.00 & 72 & 76 & 203 & 117 & 0 & 468 \\
\hline 2 & 0.94 & 17 & 28 & 47 & 53 & 0 & 145 \\
\hline 3 & 1.69 & 31 & 26 & 86 & 84 & 0 & 226 \\
\hline 4 & 2.64 & 44 & 20 & 126 & 63 & 0 & 252 \\
\hline 5 & 3.66 & 61 & 72 & 172 & 198 & 0 & 502 \\
\hline 6 & 3.79 & 68 & 54 & 192 & 108 & 0 & 422 \\
\hline 7 & 0.80 & 15 & 9 & 42 & 44 & 0 & 109 \\
\hline 8 & 2.80 & 50 & 27 & 141 & 100 & 0 & 318 \\
\hline
\end{tabular}

The total heat transfer values show segments 3 and 4 of audit 1 accrued the most heat transfer out of the other segments. This coincided with both segments experiencing venting. Segment 5 of the first audit followed close behind in terms of calculated heat transfer and was also close to venting at final experimental pressure of $1007 \mathrm{kPa}$ (146 psia), roughly $21 \mathrm{kPa}$ (3 psia) away from venting.

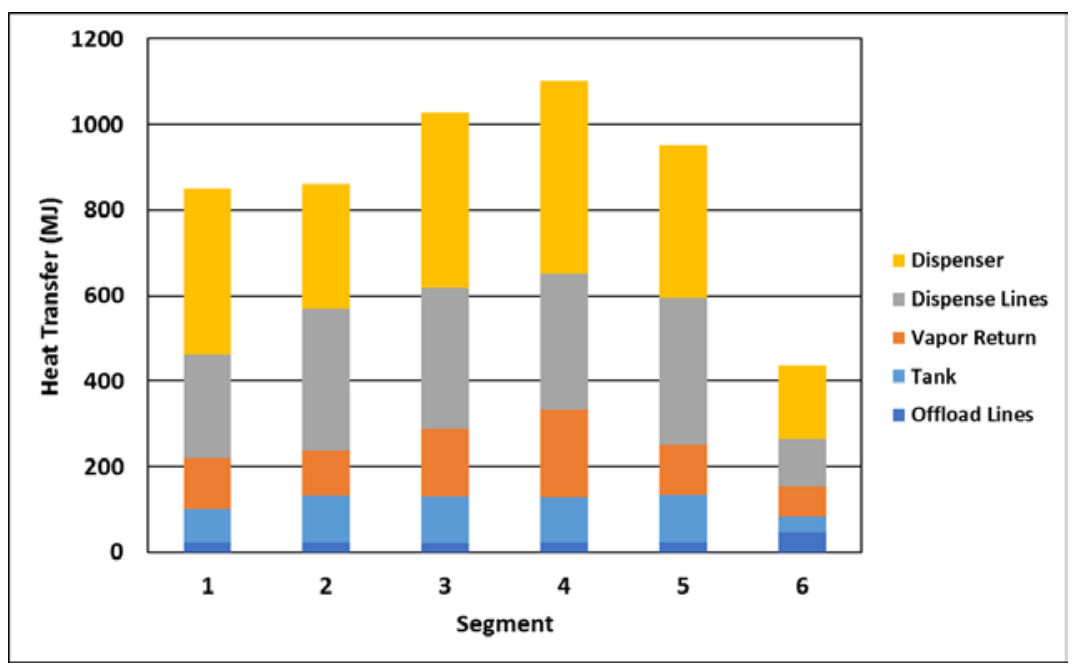

Figure 4-19: Audit 1 Total Heat Transfer by Source 


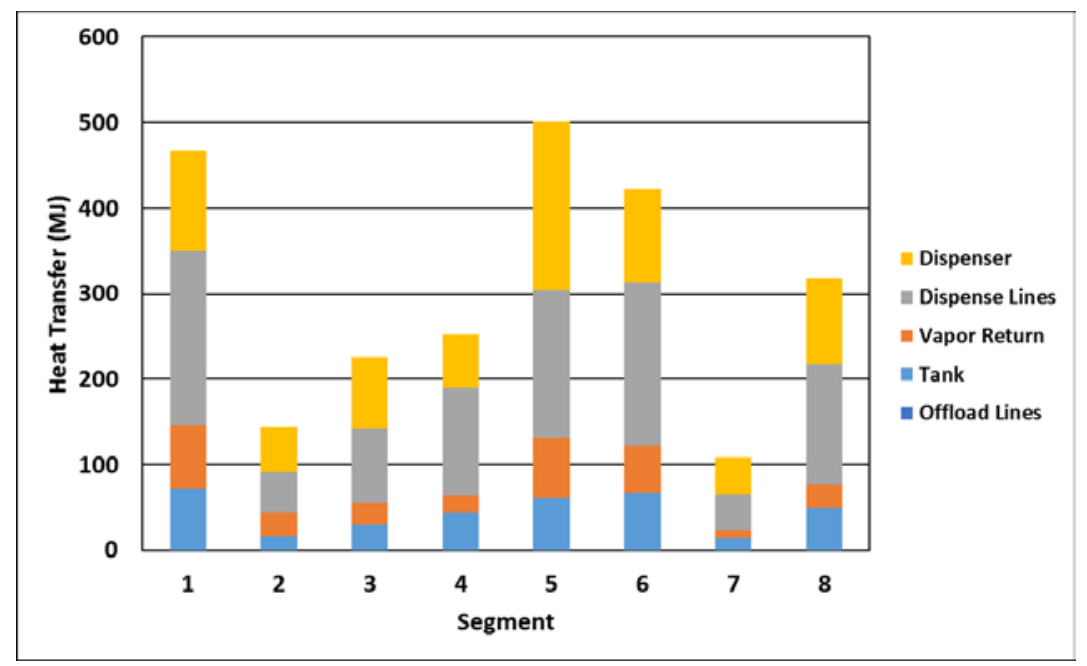

Figure 4-20: Audit 2 Total Heat Transfer by Source

Table 4-21 shows average total heat transfer values by source for each audit simulation. The values calculated were the averaged from each source over each segment of the specific audit.

Table 4-21: Audits - Average Total Heat Loads

\begin{tabular}{|c|c|c|c|c|c|c|c|}
\hline & $\begin{array}{c}\text { Duration } \\
\text { (days) }\end{array}$ & $\begin{array}{l}\text { Tank } \\
\text { (MJ) }\end{array}$ & $\begin{array}{l}\text { Vapor Return } \\
\text { (MJ) }\end{array}$ & $\begin{array}{l}\text { Dispense } \\
\text { Lines (MJ) }\end{array}$ & $\begin{array}{l}\text { Dispenser } \\
\text { (MJ) }\end{array}$ & $\begin{array}{l}\text { Offload } \\
\text { Lines (MJ) }\end{array}$ & $\begin{array}{l}\text { Total } \\
\text { (MJ) }\end{array}$ \\
\hline Audit 1 & 3.30 & 87 & 128 & 267 & 340 & 26 & 899 \\
\hline Audit 2 & 2.21 & 39 & 30 & 110 & 80 & 0 & 259 \\
\hline
\end{tabular}

Overall for the first audit station the contribution of heat through the components was $11 \%$ for the tank walls, $15 \%$ for the vapor return, 32\% for the dispense lines, 39\% for the dispenser, and 3\% for the offload pump lines. In the second audit station the contribution of the components was $15 \%$ for the tank, $13 \%$ for the vapor return, $41 \%$ for the dispense lines, $31 \%$ for the dispenser, and $0.0 \%$ for the offload pump lines. Figure 4-21 and Figure 4-22 show these results and the contribution from each source in percentage.

The first audit station was exposed to approximately 3.5 times the calculated heat transfer as the second audit station. In both cases there was a large gap between the amount heat accrued by the dispenser and dispense piping as compared to the tank and vapor return. Fleet venting rates dictated whether the vapor return or tank heat transfer was more dominant. 


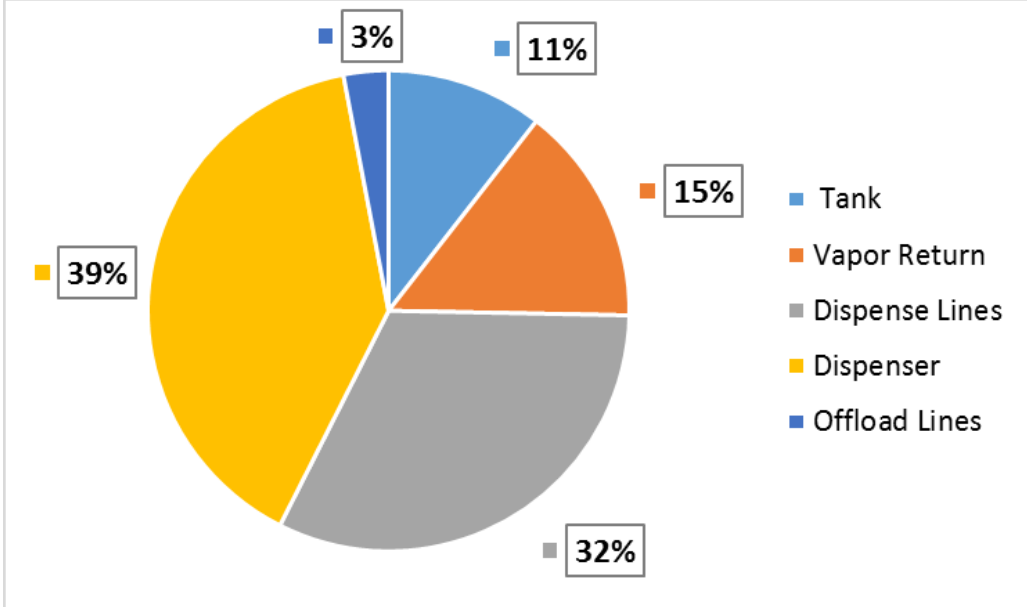

Figure 4-21: Heat Transfer by Source Over All Audit 1 Segments

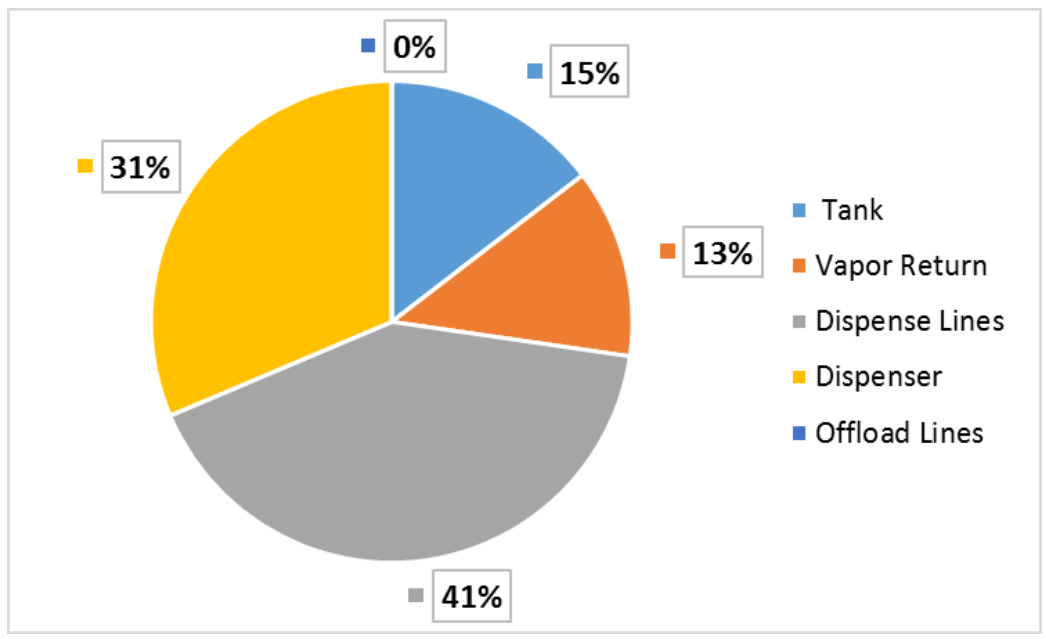

Figure 4-22: Heat Transfer by Source Over All Audit 2 Segments

Appendix C contains additional information about the pressure rise trends calculated by the model. For each segment the simulated pressure, the local ambient temperature, vehicle traffic, and time dependent heat transfer rate from each of the major heat leak sources was graphed.

\subsection{PRV Emissions and Model Comparison}

Over the course of audit 1 the station emitted methane through the main PRV during segments 3 and 4. Emissions from these two segments were investigated through data collected at the audit. The fill level at the start and end of venting was known. In addition, the vehicle traffic was also known. A mass balance was performed using the two pieces of data to determine the amount of mass emitted by the station. First the change in fill level was used to find the change of the liquid level or volume in the tank. As pressure 
was known the average pressure over the duration of the venting was used to find an average density assuming saturated conditions. The change in fill level and therefore the change in mass was subtracted by the mass dispensed to vehicles during the duration of the venting which resulted in the calculated emissions for the first audit station. Emissions from segment 3 were estimated to be $280 \mathrm{~kg}$ and from segment 4 to be $415 \mathrm{~kg}$ for a total of $695 \mathrm{~kg}$ methane emitted. The mass dispensed by the first audit station overall was $94,889 \mathrm{~kg}$ of LNG. The mass dispensed over the two segments when emissions occurred was 32,110 kg. Using these numbers the percent of mass emitted from the station over the course of the audit was $0.7 \%$ and over the course of the two segments in which venting occurred was $2.2 \%$.

At the second audit station, four manual vents were recorded with a high probability of 2 additional vent events occurring. The manual vents occurred with no vehicle dispensing during the, albeit short, duration. Pressure and fill level was known before and after the recorded vents. If the density of the LNG was assumed to be the average between the before and after pressures the mass of LNG emitted was calculated to be $1255 \mathrm{~kg}$ for the second audit station; the two additional venting events were not included in the calculation. The throughput at the station was $40,480 \mathrm{~kg}$ over the course of the audit. The vented LNG was then approximately 3.1\% of the mass dispensed to vehicles at the station.

The liquid levels at the stations were measured by hydrostatic tank gauges. Accuracy of hydrostatic gauges vary based on the pressure transducers used and were quoted as typically accurate to $+/-0.5 \%$ by mass on atmospheric tanks [55], [56]. A conservative estimate of $1 \%$ uncertainty in the sensors was used. For the audit 1 station tank, which was a 94,635 liter (25,000 gallon) tank, this equates to a +/- 946 liter (+/250 gallon) uncertainty and a +/- 568 liter (+/- 150 gallon) uncertainty for the second audit station's 56781 liter (15,000 gallon) tank. In addition, fuel transfer to the vehicles was recorded by Coriolis meters which were accurate to within $+/-0.5 \%$ by mass [57]. The uncertainty in the amount of mass vented was large based on the information available. Applying the uncertainty (+/-1.5\%) to the inventory calculation resulted in the first station venting $0.1 \%$ to $1.5 \%$ of the LNG dispensed to vehicles over the course of the audit along with the second station venting $0.9 \%$ to $5.3 \%$. Table $4-22$ summarizes the calculated emission numbers.

Table 4-22: Audit Emission Data

\begin{tabular}{|c|c|c|c|c|c|}
\hline Vent & Segment & Gallons Vented & Average Pressure (kPa) & Mass Vented (kg) & Uncertainty Range (kg) \\
\hline \multicolumn{7}{|c|}{ Audit 1 } \\
\hline 1 & 3 & 206 & 1007 & 280 & $0-619$ \\
\hline 2 & 4 & 305 & 1014 & 415 & $65-765$ \\
\hline \multicolumn{7}{|c|}{ Audit 2 } \\
\hline 1 & 1 & 190 & 496 & 277 & $58-495$ \\
\hline 2 & 2 & 150 & 490 & 219 & $0-438$ \\
\hline 3 & 3 & 241 & 558 & 348 & $131-564$ \\
\hline 4 & 7 & 282 & 503 & 411 & $192-629$ \\
\hline
\end{tabular}


Emissions from the LNG station PRV were not directly measured in any case. The accuracy of the calculations may be further compromised due to a variety of factors. Any inaccuracies or unintentional omissions in the vehicle fleet data, hysteresis of the PRV valve, and the saturated assumption were all possible sources of inaccuracy. However, the emissions values reduced from the audit data were the only available and were subsequently used to gauge the LNG station model's emission predictions.

As with the pressure rise the thermodynamic approach affected the emission prediction. Emissions were only compared for audit 1 because only the audit 1 station had natural PRV vents (non-manual). Table 4-23 shows the results from the comparison.

Table 4-23: Full Station Model Emission Prediction for Audit 1

\begin{tabular}{|c|c|c|c|}
\hline Segment & Exp. Vent $(\mathrm{kg})$ & Homog. Vent $(\mathrm{kg})$ & Stratified Vent $(\mathrm{kg})$ \\
\hline 3 & $0-619$ & 277.9 & 669.2 \\
\hline 4 & $65-765$ & 278.4 & 1086.5 \\
\hline Total & $65-1384$ & 556 & 1756 \\
\hline
\end{tabular}

The homogeneous approach calculated emissions from the station at $556 \mathrm{~kg}$ which was slightly below the $695 \mathrm{~kg}$ indicated by the data but inside the uncertainty range. The stratified approach appeared to overestimate the mass emitted by the station. The poor estimation was caused by the stratified approaches weakness in terms of handling vapor withdrawal which was evident from comparisons from audit 2 when the generator was on. As seen in other simulations vapor withdrawal causes the stratified approach to begin to increase faster in pressure instead of lowering the pressure rise rate. The increased pressure rise rate caused the venting module to activate more frequently resulting in an overestimation. The figures below show the pressure rise and behavior of the venting model.

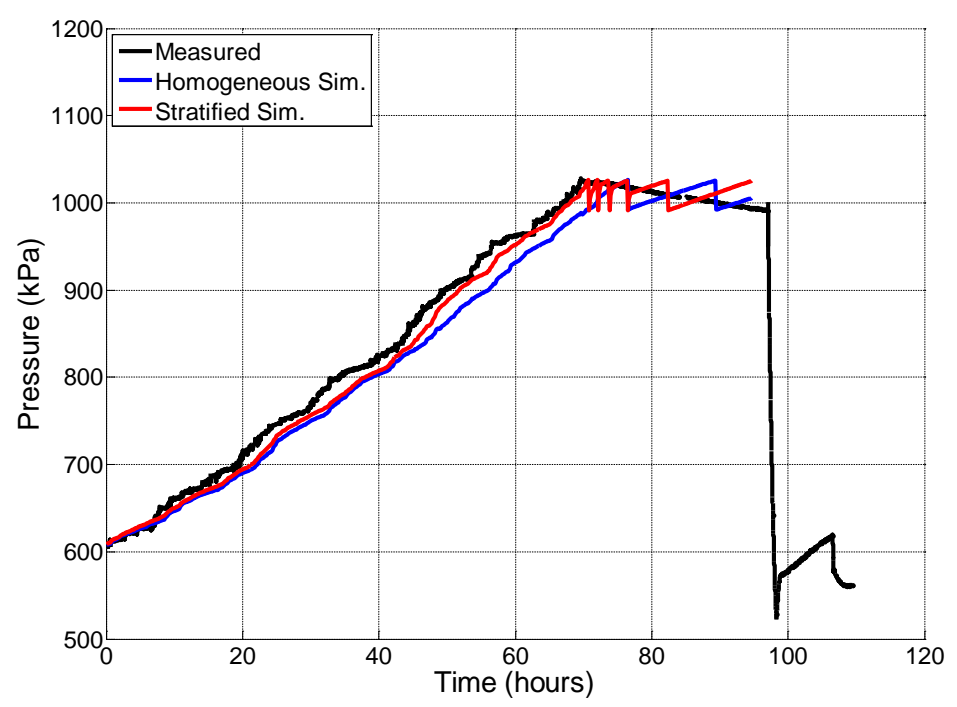

Figure 4-23: Audit 1 Segment 3 - Pressure Rise History with Venting Module Behavior 


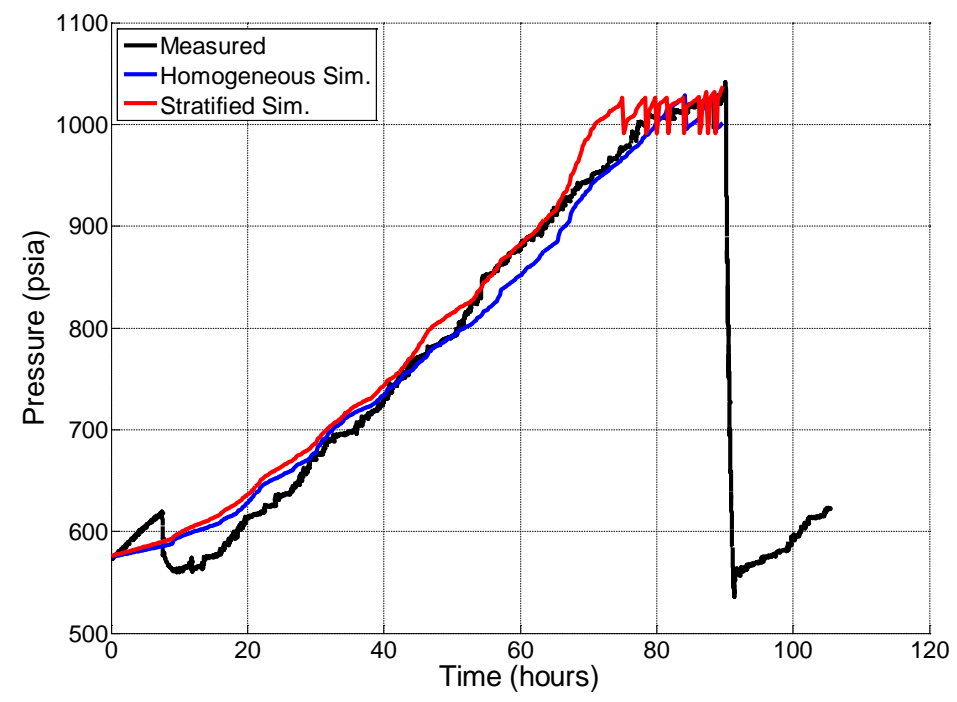

Figure 4-24: Audit 1 Segment 4 - Pressure Rise History with Venting Module Behavior

As described in the model development section the venting module dropped the pressure in the tank by a set amount of pressure below the station venting pressure. The amount of mass removed by dropping the pressure was then calculated. Once the pressure dropped the simulation continued until pressure rise reached the venting pressure again. The venting module then initiated again. At the end of the simulation time period the mass from the discrete venting events was summed to estimate emissions. The steep decreases in pressure found in the figures after the pressure reached $1025 \mathrm{kPa}$ (148.7 psia) were caused by the venting module. The pressure drop was set to $35 \mathrm{kPa}$ (5 psia) in the simulations. Testing of the venting module revealed it had on average a $1.46 \%$ error for manual vehicle vents [4]. These were vents where the initial and final pressure was known. Vents from the stations were continuous and in many cases depended on the PRV hysteresis characteristics which were unknown, therefore, the venting module utilized was a compromise. Room remains to tie the pressure rise portion of the model to more accurate models of a station tank continuous pressure relief system.

\subsection{Sensitivity Analysis}

A sensitivity analysis of the full station model was conducted for insight into the dependency of the simulations on input variables. The parameters investigated were the thermal conductivity of MLI used throughout the station, average ambient temperature, and piping lengths for the dispense piping. These variables were used to investigate their effect on pressure rise and emissions. In addition, sensitivity of the venting module was investigated by varying the amount of pressure the module assumed was vented by 
each iteration of the venting module. The parameters were chosen to highlight important variables in the model but more inputs exist, therefore, the analysis was not exhaustive. The analysis was performed for the full station model utilizing the homogeneous thermodynamic approach. Only the dimensions and inputs used for the sensitivity analysis were changed. The data from segment 1 of the first audit was used for the pressure rise analysis and segment 3 was used for the sensitivity of the emissions. Each variable for the pressure rise was first analyzed by varying the input values by $+/-10 \%$ and $+/-20 \%$. In certain cases the 10 to 20 percent change was unrealistic and/or highly unlikely such as a $20 \%$ modification of the ambient temperature. A second set of simulations was then run with the values set to a range considered more realistic for the data collected. The section finishes with a discussion of the confidence and variability of the model.

\subsubsection{Pressure Rise Sensitivity}

The first sensitivity simulations run investigate the pressure rise change due to percent variation of the input values. Figure 4-25 shows the pressure rise rate variations in the model from modifying the average ambient temperature by percentages for the segment. The red line indicates a $20 \%$ increase in the ambient temperature used by the model with the yellow, green, and cyan lines representing a 10\% increase, $20 \%$ decrease, and $10 \%$ decrease in the ambient temperature, respectively. The blue line is the original model simulation the results of which were presented in the previous section. Again, the black line represents the actual experimental pressure measure for the segment of the audit. The format is the same for the subsequent plots in this section.

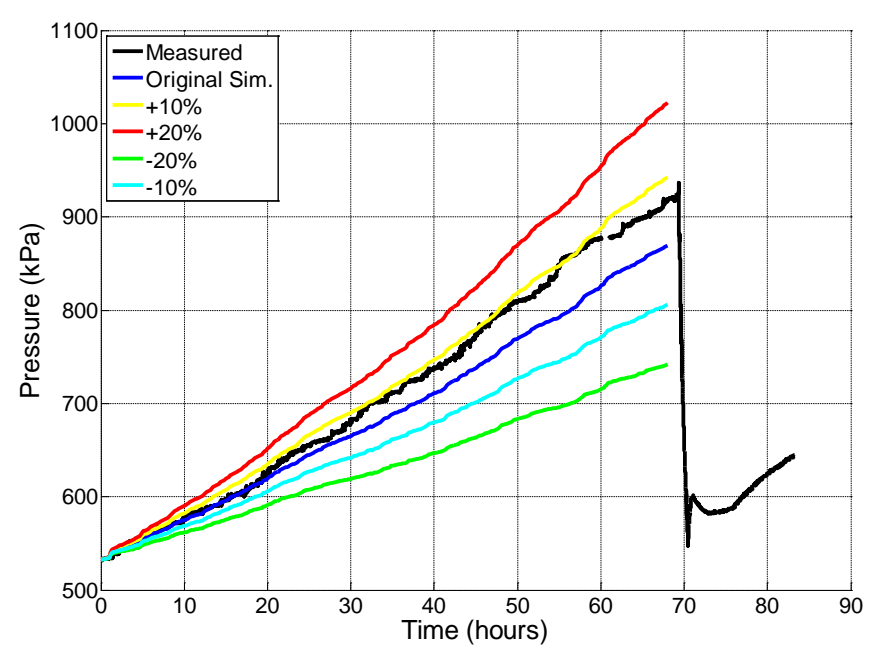

Figure 4-25: Audit 1 Seg. 1 - Tank Pressure Rise Sensitivity to Ambient Temperature Modified by a Precentage Decrease and Increase of the Average 
Table 4-24 summarizes the simulation pressure variations from the modification of the input ambient temperature. The pressure rise trend column in the table shows the source and or modification of the input variable. The final pressure column details the end pressure of the simulations or experimental data for comparison against the original simulation final pressure in the pressure difference column. The pressure difference column shows the change in pressure from the variable modifications and the original difference between the model and the experimental data. The percent change column is calculated using relative error with the original simulation final pressure as the reference point.

The average temperature for the segment was $290 \mathrm{~K}$, therefore a 20 percent change increased or decreased the temperature by $58 \mathrm{~K}$. A temperature swing of that magnitude was not physically probable, however, it allows comparison in terms of percent change between the other parameters. Table 4-24 presents a summary of final pressure change from varying the ambient temperature by $10 \%$ and $20 \%$. Pressure change was not linear and the pressure rise increased more than it decreased. The $10 \%$ increase and decrease (29 K) was considered an extreme variation and resulted in an approximately $69 \mathrm{kPa}(10 \mathrm{psia})$ change.

Table 4-24: Audit 1 Seg. 1 - Percent Variation of Ambient Temperature Sensitivity Summary

\begin{tabular}{|l|r|r|r|}
\hline \multicolumn{1}{|c|}{ Pressure Rise Trend } & Final Pressure $(\mathrm{kPa})$ & \multicolumn{1}{c|}{ Pressure Difference $(\mathrm{kPa})$} & Percent Change (\%) \\
\hline Experimental Data & 919 & 50 & \\
\hline Original Sim. & 869 & 0 & \\
\hline $10 \%$ & 943 & 73 & $8.4 \%$ \\
\hline $20 \%$ & 1022 & 153 & $16.2 \%$ \\
\hline$-20 \%$ & 742 & -128 & $-12.5 \%$ \\
\hline$-10 \%$ & 806 & -63 & $-8.5 \%$ \\
\hline
\end{tabular}

The assumed thermal conductivity of multilayer insulation used in the model was $5 * 10^{-5} \mathrm{~W} /(\mathrm{m} * \mathrm{~K})$. Figure 4-26 shows the pressure rise change from modifying the thermal conductivity of MLI by $10 \%$ and $20 \%$. The percent change elicited low changes in the final pressure prediction. 


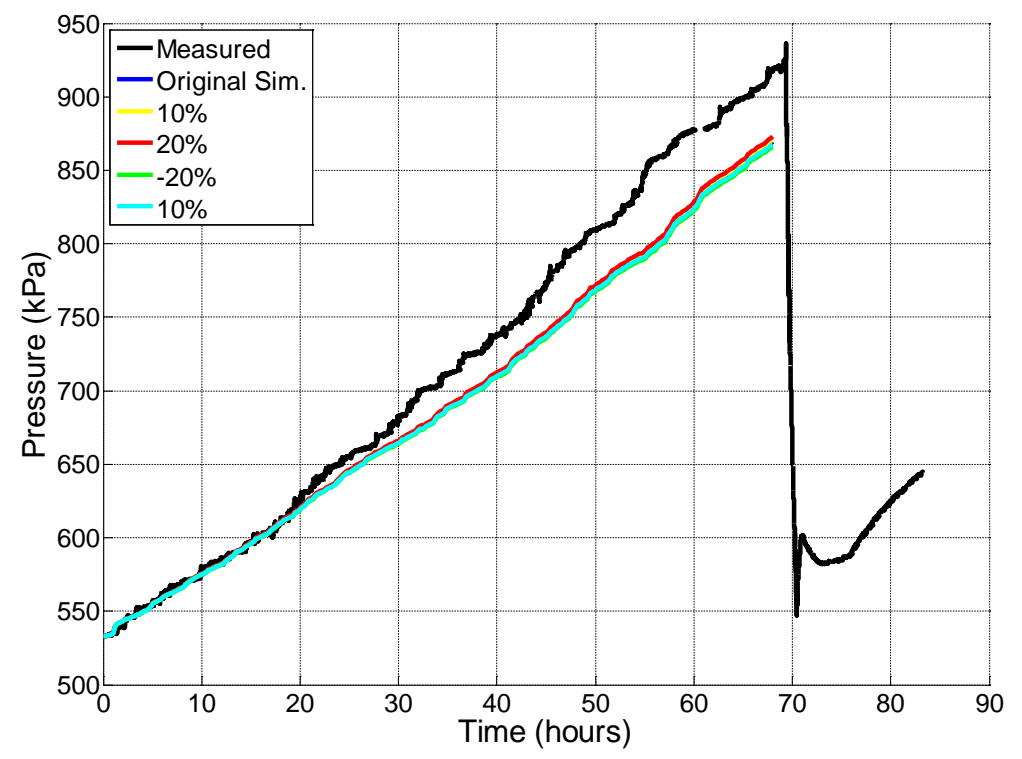

Figure 4-26: Audit 1 Seg. 1 - Sensitivity to MLI Thermal Conductivity

Table 4-25 summarizes the changes in pressure from the variation in thermal conductivity of the multilayer insulation used in the station. A $10 \%$ percent change in the multilayer insulation resulted in a $1.4 \mathrm{kPa}(0.2 \mathrm{psia})$ change and a $20 \%$ variation yielded a $3.4 \mathrm{kPa}$ (0.5 psia) change.

Table 4-25: Audit 1 Seg. 1 - Percent Variation of MLI Thermal Conductivity Summary

\begin{tabular}{|l|r|r|r|}
\hline \multicolumn{1}{|c|}{ Pressure Rise Trend } & Final Pressure $(\mathrm{kPa})$ & \multicolumn{1}{c|}{ Pressure Difference (kPa) } & Percent Change (\%) \\
\hline Experimental & 919 & 50 & 0 \\
\hline Original Sim. & 869 & 1.4 & 0.2 \\
\hline $10 \%$ & 871 & 3.4 & 0.4 \\
\hline $20 \%$ & 873 & -3.4 & -0.4 \\
\hline$-20 \%$ & 866 & -1.4 & -0.2 \\
\hline$-10 \%$ & 867 & $20 \% . A 11$ piping types and \\
\hline
\end{tabular}

The dispense piping system lengths were also varied by $10 \%$ and $20 \%$. All piping types and sizes were varied by these percentages. Accurate dimensions were provided for the audit 1 station, therefore a $20 \%$ variation in piping lengths was considered unlikely. A $10 \%$ percent inaccuracy in the piping dimensions was also considered unlikely but possible. However, in the case of the second audit station a $20 \%$ variations in the input piping lengths was possible if not likely. Results for the sensitivity analysis are shown in Figure 4-27. 


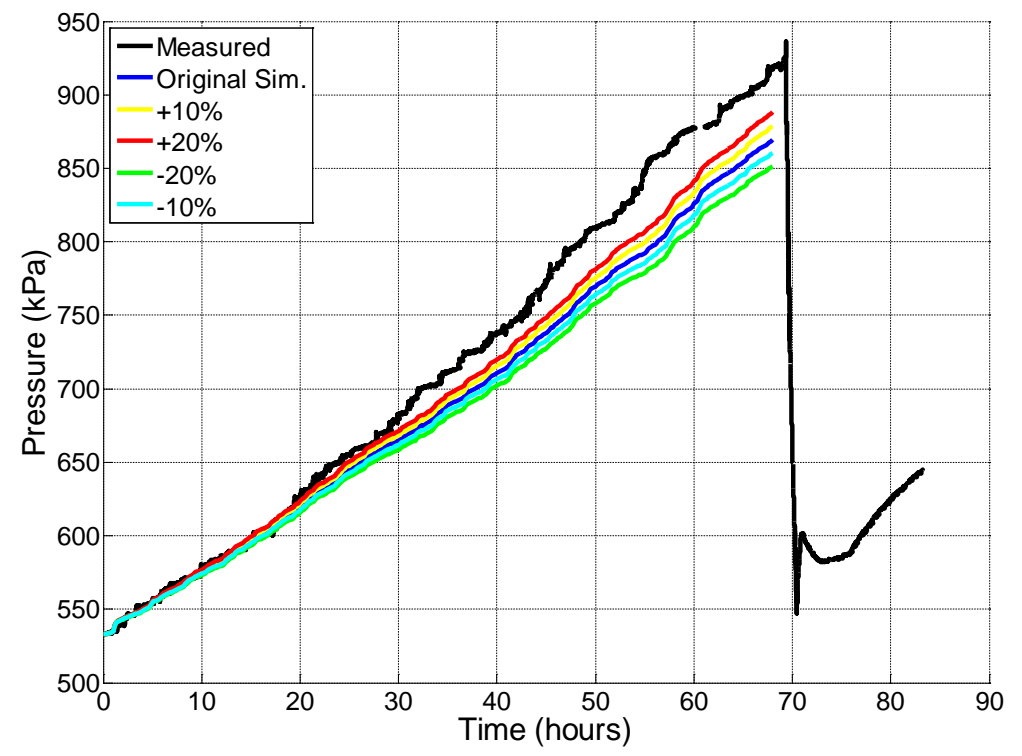

Figure 4-27: Audit 1 Seg. 1 -Variation of Dispense Piping Lengths Summary

Table 4-26 shows the final pressures, pressure differences, and the percent change relative to the original simulation predicted final pressure due to the parameter variations. A $20 \%$ increase in the length of piping caused the simulation to increase the final predicted pressure by $2.2 \%$ ( 2.7 psia). Likewise, a decrease in the piping lengths, which would cause a decrease in the amount of energy transfer calculated, caused a $2 \%$ decrease in the final predicted pressure.

Table 4-26: Audit 1 Seg. 1 -Variation of Dispense Piping Lengths Summary

\begin{tabular}{|l|r|r|r|}
\hline \multicolumn{1}{|c|}{ Pressure Rise Trend } & Final Pressure $(\mathrm{kPa})$ & Pressure Difference $(\mathrm{kPa})$ & Percent Change (\%) \\
\hline Experimental & 919 & 50 & \\
\hline Original Sim. & 869 & 0 & 1.2 \\
\hline $10 \%$ & 879 & 10 & 2.2 \\
\hline $20 \%$ & 888 & 19 & -2.0 \\
\hline$-20 \%$ & 852 & -18 & -1.1 \\
\hline$-10 \%$ & 860 & -9 & \\
\hline
\end{tabular}

The variations of the ambient temperature, thermal conductivity, and piping lengths highlighted the model's sensitivity to these input variables. As the input variables investigated were modified by percentages relative to the original input values, changes in ambient temperature were found to shift the pressure rise prediction in the model the greatest. Variations of the length of piping had the second greatest effect with changes in the thermal conductivity of the multilayer insulation having the least effect on the final pressure prediction. While shifting the variables by a set percentage is illustrative of the strength of 
certain inputs relative to others it is also illuminating to modify the variables by more realistic values. Next the ambient temperature and thermal conductivity will be varied by some values considered to be possible variations in the input data. This was already done in the pipe length sensitivity.

The average ambient temperature was varied by $+/-5 \mathrm{~K}$ and by $+/-10 \mathrm{~K}$, and considered a more realistic variation in temperature for the station. Figure 4-28 shows the change in simulated pressure rise from the varying average ambient temperatures. A 5 Kelvin change in the ambient temperature was considered likely indicative of the uncertainty in the temperature data. As discussed earlier many weather stations have a temperature uncertainty of at best $2{ }^{\circ} \mathrm{C}$ [53]. In addition, the temperature stations used for the temperature data input were local but more than 5 miles away deeming a $5 \mathrm{~K}$ deviation in the actual temperature experience by the station reasonable.

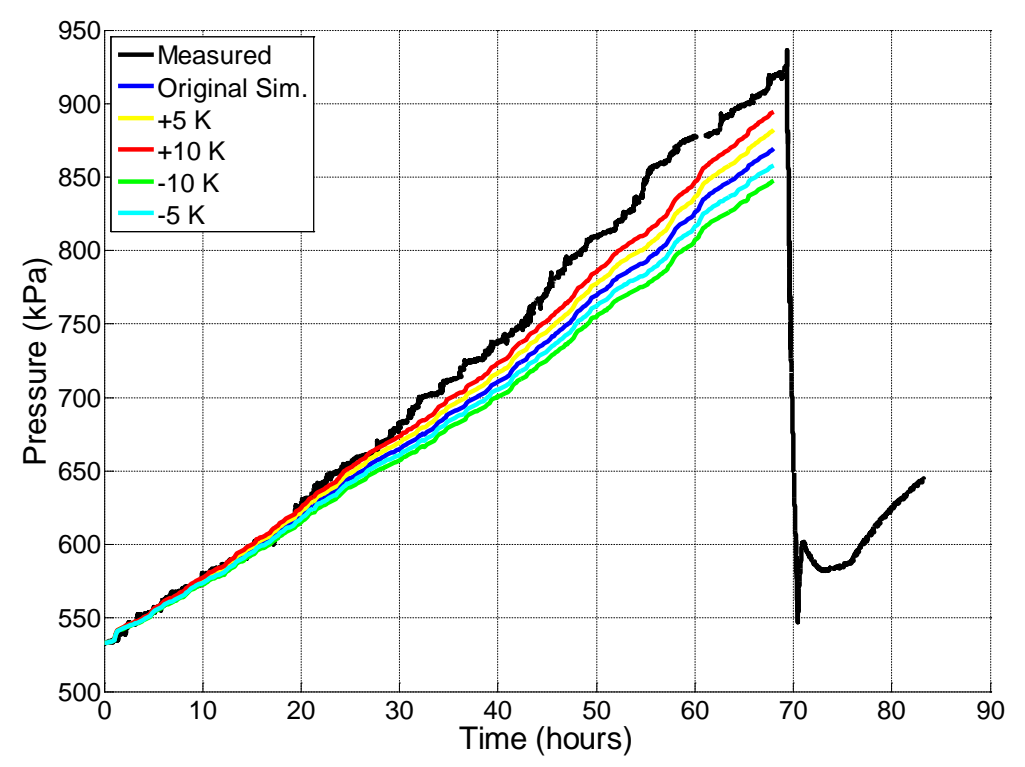

Figure 4-28: Audit 1 Seg. 1 - Sensitivity of Ambient Temperature

Table 4-27 shows the change in final pressure relative to the original simulation results. A rise in temperature increased the final simulated pressure as expected. The 5 kelvin variation of the ambient temperature yielded an approximately $12 \mathrm{kPa}$ (1.8 psia) change in the final pressure results. 
Table 4-27: Audit 1 Seg. 1 - Variation of Ambient Temperature Summary

\begin{tabular}{|l|r|r|r|}
\hline \multicolumn{1}{|c|}{ Input Variation } & Final Pressure $(\mathrm{kPa})$ & \multicolumn{1}{|c|}{ Pressure Difference $(\mathrm{kPa})$} & Percent Change (\%) \\
\hline Experimental & 919 & 50 & \\
\hline Original Sim. & 869 & 0 & 1.4 \\
\hline $5 \mathrm{~K}$ & 882 & 12 & 2.9 \\
\hline $10 \mathrm{~K}$ & 894 & 26 & -2.5 \\
\hline$-10 \mathrm{~K}$ & 847 & -22 & -1.4 \\
\hline$-5 \mathrm{~K}$ & 858 & -12 & \\
\hline
\end{tabular}

Thermal conductivity of the MLI was varied by 2 times, 5 times, $1 / 5^{\text {th }}$, and $1 / 2$ the assumed value. The midrange variation of the thermal conductivity ( 2 times and 1/2) was considered a realistic variation for a station. Variations in manufacturing quality, layer spacing used and differing boundary temperatures on the station components were likely to cause these variations. A 5 times increase in the thermal conductivity was considered unlikely but possible. A reduction to $1 / 5^{\text {th }}$ the thermal conductivity was also considered unlikely. Due to recent advances future stations may be able to achieve the reduced insulation thermal conductivity [23]. Figure 4-29 shows the pressure rise for the variation in thermal conductivity.

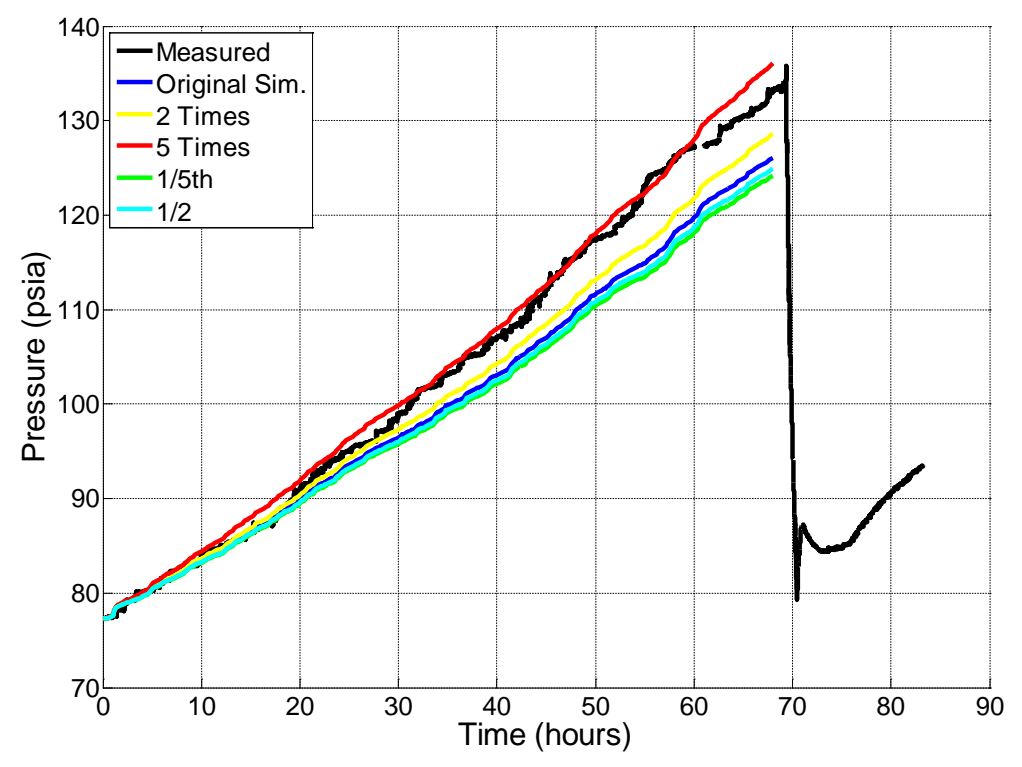

Figure 4-29: Audit 1 Seg. 1 -Sensitivity to MLI Thermal Conductivity

Table 4-28 summarizes the sensitivity study of the multi-layer insulation thermal conductivity change and its effect on the final predicted pressure. An increase in the thermal conductivity of the MLI resulted in a large increase in the final pressure of the station. However, a decrease of the same amount dropped the final station pressure by a lesser amount. As this sensitivity shows the multilayer insulation 
thermal performance was already effective and any further reductions resulted in minimal gains. A 2 times increase in the thermal conductivity resulted in a $17 \mathrm{kPa}(2.5 \mathrm{psia})$ increase in the final pressure while a reduction of $1 / 2$ the thermal conductivity yielded an $8 \mathrm{kPa}(1.2 \mathrm{psia})$ drop.

Table 4-28: Audit 1 Seg. 1 -Sensitivity Study Variation of MLI Thermal Conductivity Summary

\begin{tabular}{|l|r|r|r|}
\hline \multicolumn{1}{|c|}{ Input Variation } & Final Pressure (kPa) & Pressure Difference $(\mathrm{kPa})$ & Percent Change (\%) \\
\hline Experimental & 919 & 50 & \\
\hline Original Sim. & 869 & 0 & 1.9 \\
\hline 2 Times & 887 & 17 & 7.7 \\
\hline 5 Times & 938 & 68 & -1.4 \\
\hline $1 / 5$ th & 856 & -13 & -1.0 \\
\hline $1 / 2$ th & 861 & -8 & \\
\hline
\end{tabular}

The variables altered by the selected amounts reflect an estimate of the uncertainty in the input data which highlights an incomplete but illustrative confidence for the model pressure rise prediction. A variation in the thermal conductivity of the multilayer insulation of 2 times and $1 / 2$ was considered realistic along with a variation in the input piping lengths of $+10 \%$ and $-10 \%$, and a variation of $+5 \mathrm{~K}$ and $-5 \mathrm{~K}$ in ambient temperature. Using the results from the sensitivity analysis this gives the model a preliminary confidence interval of $+39 \mathrm{kPa}$ and $-29 \mathrm{kPa}$ around the model predicted final pressure. The pressure changes due to variations in the input data not only effects the final pressure prediction of the model but impacts the emission calculation of the process model. Since the emission estimation of the PRV started when the station tank reached a specific pressure the shift in pressure rise rate caused the process model to begin calculating emissions before or after the actual vent time. The effects of this are investigated in the Emission Sensitivity section in more detail.

\subsubsection{Emission Sensitivity}

The emission estimate made by the model also underwent a sensitivity analysis. Parameters were varied by the considered realistic/possible values the same as found in the pressure rise sensitivity analysis. Additionally, the pressure drop assumption used in the venting module was also investigated. As described in the model development section the venting module dropped the pressure in the tank by a set amount of pressure below the station venting pressure. The amount of mass removed by dropping the pressure was then calculated. Once the pressure dropped the simulation continued until pressure rise reached the venting pressure again. The venting module then initiated again. At the end of the simulation time period the mass from the discrete venting events was summed to estimate emissions. Segment 3 of audit 1 was used for the analysis as station venting occurred. 
Figure 4-30 shows the pressure rise and venting behavior of the model to changing dispense piping lengths. The sharp decreases of pressure at the end of the simulation was the venting module's behavior and the figure shows the typical response of the venting model to varying parameters. If the variable was increased and tended to increase the energy transfer rate, the pressure rise rate increased enough that the venting module initiated more times resulting in a higher emissions estimation. In some cases a change in pressure rise rate would not result in a different prediction because the change was not enough to alter the number of times the module activated. The venting module dropped the pressure by a set amount, predetermined at the start of the simulation, and calculated the amount of mass vented for the pressure drop. Pressure rise was then calculated normally from the new pressure until it reached the PRV pressure. A 34 $\mathrm{kPa}$ (5 psia) pressure drop was used for the original simulations.

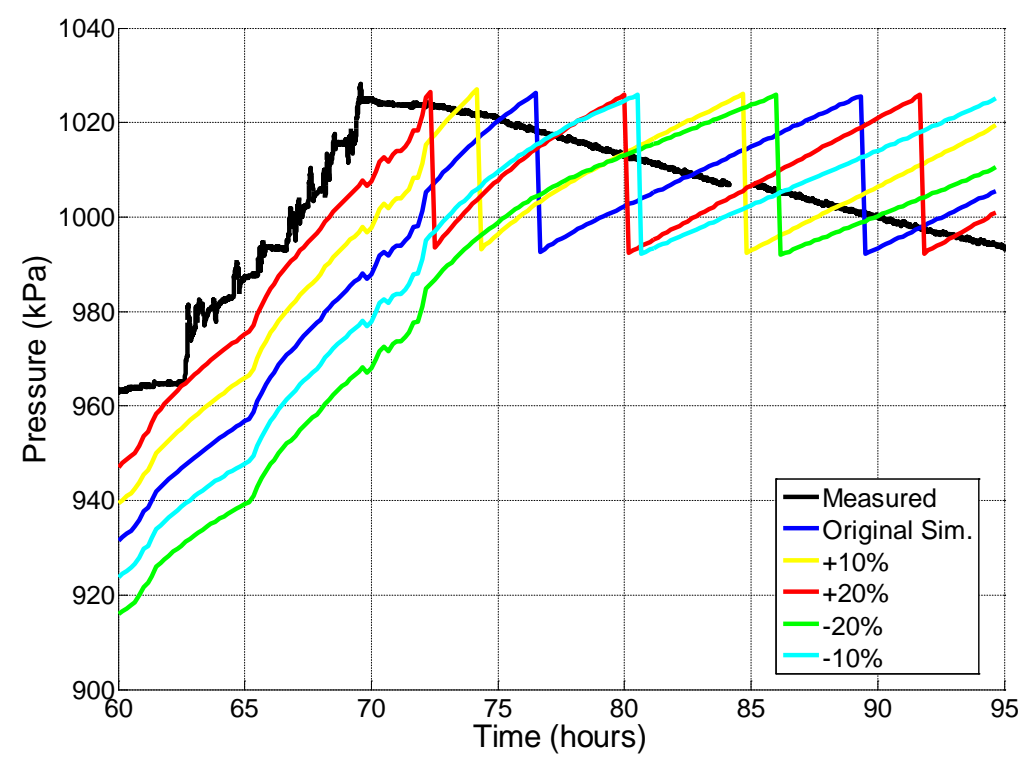

Figure 4-30: Audit 1 Seg. 3 - Dispense Piping Lengths Venting Module Sensitivity Pressure Trends

Table 4-29 presents the summary of emission results for the variations in dispense piping lengths. Emissions varied substantially when the piping lengths were changed. Large changes were due to the interaction between the venting module and the pressure rise. A change in pressure rise rate resulted in the tank venting earlier or later, increasing or reducing the number of times the venting module was activated. The number of time the venting module was activated caused the changed emission estimation throughout the sensitivity analysis.

The input variation column show the variation in the input variable being discussed by the table and includes the results from the original simulation which is considered the reference point for the emission 
difference and percent difference columns. The percent difference was calculated using the relative error equation with the original simulation's PRV emission as the reference.

Table 4-29: Dispense Piping Lenghts Emission Sensitivity Summary

\begin{tabular}{|r|r|r|r|}
\hline \multicolumn{1}{|c|}{ Input Variation } & Modeled PRV Emission (kg) & Emission Difference (kg) & Percent Difference (\%) \\
\hline Original Sim. & 278 & 0 & $0.0 \%$ \\
\hline $10 \%$ & 281 & 3 & $1.0 \%$ \\
\hline $20 \%$ & 414 & 136 & $49.1 \%$ \\
\hline$-20 \%$ & 140 & -138 & $-49.7 \%$ \\
\hline$-10 \%$ & 139 & -139 & $-50.0 \%$ \\
\hline
\end{tabular}

Table 4-30 shows the summary of results for the MLI thermal conductivity variations. As with the dispense piping the emission values differed greatly, by as much as $156 \%$. A similar prediction to the original simulation was achieved by the $1 / 2$ variation in thermal conductivity due to the specific simulation initiating the venting module the same number of times as the original simulation.

Table 4-30: Thermal Conductivity of MLI Emission Sensitivity Summary

\begin{tabular}{|l|r|r|r|}
\hline \multicolumn{1}{|c|}{ Input Variation } & \multicolumn{1}{|c|}{ Modeled PRV Emission (kg) } & Emission Difference (kg) & \multicolumn{1}{c|}{ Percent Difference (\%) } \\
\hline Original Sim. & 278 & 0 & $0.0 \%$ \\
\hline 2 Times & 419 & 141 & $50.8 \%$ \\
\hline 5 Times & 713 & 435 & $156.5 \%$ \\
\hline $1 / 5$ th & 139 & -139 & $-50.0 \%$ \\
\hline $1 / 2$ & 279 & 1 & $0.3 \%$ \\
\hline
\end{tabular}

Table 4-31 shows the summary for the sensitivity analysis results of emissions from changing the average ambient temperature. A rise in temperature increased the pressure rise rate and thereby the emission estimation while a decrease in temperature served to lower the emission estimation. For the $5 \mathrm{~K}$ rise in temperature the pressure rise rate did not increase enough to cause the venting module active again, therefore the emission prediction is similar to the original simulation.

Table 4-31: Average Ambient Temperature Emission Sensitivity Summary

\begin{tabular}{|l|r|r|r|}
\hline \multicolumn{1}{|c|}{ Input Variation } & Modeled PRV Emission (kg) & Emission Difference (kg) & Percent Difference (\%) \\
\hline Original Sim. & 278 & 0 & $0.0 \%$ \\
\hline $5 \mathrm{~K}$ & 279 & 1 & $0.3 \%$ \\
\hline $10 \mathrm{~K}$ & 418 & 140 & $50.4 \%$ \\
\hline$-10 \mathrm{~K}$ & 139 & -139 & $-50.0 \%$ \\
\hline$-5 \mathrm{k}$ & 139 & -139 & $-50.0 \%$ \\
\hline
\end{tabular}


Next the original simulation was modified by changing the assumed pressure drop of the venting module. The initial pressure drop was set to $34 \mathrm{kPa}$ (5 psia). Table 4-32 presents the results from the sensitivity analysis on the venting module assume pressure drop. In all but one case the emissions prediction lowered. The average absolute percent change of varying the assumed pressure drop was 19.7 percent.

Table 4-32: Vent Model Pressure Drop Emission Sensitivty Summary

\begin{tabular}{|l|r|r|r|}
\hline $\begin{array}{c}\text { Vent Model } \\
\text { Pressure Drop (kPa) }\end{array}$ & $\begin{array}{c}\text { Modeled PRV Emission } \\
(\mathrm{kg})\end{array}$ & $\begin{array}{c}\text { Emission Difference } \\
(\mathrm{kg})\end{array}$ & $\begin{array}{c}\text { Percent Difference } \\
(\%)\end{array}$ \\
\hline Original Sim. (34 kPa) & 278 & 0 & $0.0 \%$ \\
\hline 62 & 254 & -24 & $-8.7 \%$ \\
\hline 55 & 226 & -52 & $-18.6 \%$ \\
\hline 48 & 198 & -80 & $-28.6 \%$ \\
\hline 41 & 339 & 61 & $-17.7 \%$ \\
\hline 28 & 229 & -49 & $-7.7 \%$ \\
\hline 21 & 256 & -21 & $-17.2 \%$ \\
\hline 14 & 230 & -48 & $-26.1 \%$ \\
\hline 7 & 205 & -73 & \\
\hline
\end{tabular}

Overall the emission prediction varied by a substantial margin. The pressure drop assumption the venting module used produced an average absolute change from the original simulation of $18.3 \%$. Averaging all the absolute changes in the sensitivity analysis resulted in an average percent change of $37 \%$. If only the physical parameters effecting pressure rise were average the result was an average $47 \%$ change in emissions from the original simulation. The time when the tank pressure reached the venting pressure of the station was the dominant factor effecting emission estimation for the current formulation of the venting module. If the pressure predicted by the model reached the vent pressure before the actual station pressure ( over predicted pressure rise rate) then an increase in the amount of emissions calculated was likely to occur and the opposite was true for an under prediction in the pressure rise rate. The process model under predicted pressure rise on the first audit station consistently and therefore was most likely under estimating the amount of mass emitted from the station PRV. 


\section{Conclusions and Discussion}

First, an energy transfer model was created to calculate the heat transfer and energy transfer of station components to the cryogenic fluid contained in a liquefied natural gas fueling station. The energy transfer model was then incorporated in a full process model which simulated interactions of the station with vehicles and station pressure relief valve emissions. Data collected at WVU CAFFEE on stationary vehicle tanks such as hold time pressure rise trends and normal evaporation rates for the tanks were presented. The information from this was used to investigate two thermodynamic models utilized in the process model as well as the energy transfer model's calculation for heat transfer through the cryogenic storage tank wall. Information collected from two operational LNG stations were then presented. Comparison of the full station process model to the collected LNG station pressure history was then performed. The model was compared to the pressure rise rate and final pressure of each segment and absolute relative errors of the simulations were presented. Next, estimations for emissions from the pressure relief valve on both stations and calculation of emissions from the model were described. Finally, a preliminary sensitivity analysis on the model was performed to highlight specific important parameters effect on the pressure rise and emission predictions of the full station model. A discussion of each of these facets of the study follows.

\subsection{Data and Results Discussion}

The hold time test comparisons performed on the vehicle tanks revealed the necessity of modeling thermal stratification and the rate of evaporation or condensation at the liquid-vapor interface. The homogeneous approach performed well at almost full levels but accuracy deteriorated quickly at fill levels below $80 \%$, from a $12.2 \%$ error in pressure rise rate at $88 \%$ fill to a $474 \%$ error at $28 \%$ fill. The stratified approach was able to predict the final pressure to within $20 \%$ on average for comparison periods of over a week of real time. In addition, LNG cryogenic tanks were verified to hold fuel at normal operating pressures, $896 \mathrm{kPa}$ (144.7 psia) or greater, for over a week until venting occurred. Idleness of LNG fueled vehicles over a weekend or holiday, therefore, was considered unlikely to attribute methane emissions.

Normal evaporation rate tests verified literature research showing the evaporation rate lowers as fill level lowers. It was evident through current literature and experience with the vehicle tank experiments, the normal evaporation process depended on natural convection in both the liquid and vapor spaces, the surface area of the tank exposed to the liquid LNG, and dynamics at the liquid-vapor interface. Experience with the stratified model indicated the amount of heat transferred to the stored LNG changed over time as 
stratification developed. In addition, pressure rise or boil off rate at the liquid-vapor interface was dominated by the amount of energy entering the liquid dictated by the fill level.

Simulations compared to the data collected at LNG stations showed a different result from the hold time tests. The homogenous thermodynamic approach performed well on the full station tank and was able to handle certain cases the stratified approach did not. In general, the stratified approach outperformed the homogeneous approach in terms of pressure rise with an average absolute error between the two audits of $16.5 \%$ for the stratified and $18.6 \%$ for the homogeneous. However, at audit 2 some time periods involved no vehicle traffic with an active pressure mitigation strategy. During the time periods of segment 1 and segment 6 the pressure in the station tank fell. The stratified model was not able to capture the behavior and instead predicted a pressure rise. Overall both thermodynamic approaches performed better for the audit 1 station due to the greater amount of information available for the station. The average absolute error for the stratified approach was $7.5 \%$ and the average for the homogeneous approach was $10 \%$. At the second audit station only estimates of the lengths and sizes of the plumbing were available from visual inspection; no schematics were provided or available. Therefore, the absolute errors were larger with $25.7 \%$ for the stratified approach and $25.1 \%$ for the homogeneous approach. During the course of the study the initial goal was to create a model which predicted pressure rise to within $20 \%$. This was considered by industry partners to constitute a model accurate enough to estimate emissions and capture the dynamics of the modeled LNG stations. The first station easily met this requirement. The lack of precise dimensional data for the second station and not the formulation of model was the reason the second station did not meet the performance goal.

The station at audit 2 appeared to vent at least $0.9 \%$ and up to $5.3 \%$ of its product over the duration and was the largest emitter from the study. The first audit station vented over two segments for total of approximately $0.1 \%$ to $1.5 \%$ of its dispensed product. The model for station 1 indicated emissions from the first audit station to be approximately $0.6 \%$ of the fuel dispensed over the 21 day period. Applying the limits of the preliminary sensitivity analysis the first station vented $0.4 \%$ to $0.8 \%$ of the LNG dispensed. From the sensitivity analysis performed on the PRV model the model emission calculation is quite sensitive to the onset of venting and the initial assumption of pressure drop. Further refinements are necessary to improve predicting power but a useful estimate can be calculated by the current approach.

The significance of the emissions are seen when comparing to literature on fuel switching [1]. Methane is considered to have many times the greenhouse warming potential of carbon dioxide. However, methane produces less carbon dioxide when burned giving it an advantage when used as a fuel. Any emissions of unburned methane eroded its greenhouse gas advantage over more common fuels such as gasoline, diesel, and coal. This is complicated by the fact that methane breaks down into carbon dioxide naturally in the atmosphere. Thus, the use of methane produces a greenhouse warming benefit with the 
caveats of the benefits being delayed and the near term global warming potential being higher. Therein a tipping point exists where switching from conventional fuels to natural gas, if natural gas emissions are high, brings delayed benefits and a short term increase in greenhouse warming potential. Alvarez et al. identified some of these points for transportation vehicles, heavy duty vehicles, and power plants. For transportation vehicles less than $1.6 \%$ of methane produced must be emitted, otherwise any fuel switching will not bring benefits for many years. It is $1 \%$ for heavy duty vehicles and 3.2\% for power plants. At the time of the study the emissions were estimated (based on data from the EPA) to be $3 \%$ from natural gas systems. With $2.4 \%$ lost from well to local distribution infrastructure and $0.6 \%$ lost during vehicle refueling and from the vehicle itself. The $0.6 \%$ emission rate is the quantity comparable to the emissions seen in this study. The emission rates from station PRVs seen in this study can be quite significant. As of now the low number of stations mean venting is not an issue. An increase in the number of stations may make it an issue depending on the number of stations actually venting. Even with the available amount of information garnered from this study for emissions from station tank PRVs, information is too scarce to make any broad assertions about venting rates from LNG stations. For example experience with the model and through the study indicated these emissions are likely zero at stations with optimal vehicle traffic. Therefore, to determine emissions on the whole from this source will require precise knowledge of the number of vehicles and stations present in the industry.

Other sources of emissions also exist at a station and while not characterized in this study bear mentioning. A tanker was witnessed to refuel the station by connecting a standard 20 foot, 4 inch diameter hose to the station offload lines. After refueling was complete the LNG inside the hose was dumped out. In addition, vehicles which arrived at the station sometimes vented to atmosphere before fueling. The practice was used by drivers because it lowered the overall fueling time, especially if a vent back to the station was necessary to refuel. Only a handful of cases were observed at the audits but interviews of drivers indicated it was a common practice if they were under time constraints. While a potentially large source of emissions the extent of the practice was not investigated in this study.

Overall, from the experience of the two audits, it was possible to predict the pressure rise of a station to within $7.5 \%$ based on pressure rise rate. However, only an approximation for the emissions from a LNG station was capable of being predicted, based on the sensitivity analysis which showed the assumption for the pressure drop in the venting module alone shifted results for emissions $+/-18.3 \%$ on average. Experience with the simulations and discussions with station owners indicated the fuel throughput at the station was the biggest factor in determining whether a station vented or not. All evidence points toward venting at underutilized stations occurring due to highly variable local conditions. For instance, the segments when venting occurred for the first audit saw the most vehicle traffic; this was contrary to the notion that more vehicles will reduce the risk of venting. A large degree of vehicles venting back to the 
station was a possible cause. From analysis of the experimental data it was shown the station's pressure rise rate increased with increasing vehicle activity. However, as a result of insight from the energy transfer analysis it was apparent the thermal load on the station would peak after a threshold amount of vehicle traffic. At higher amounts of vehicle traffic, the station would not vent because the fuel rate dispensed would allow for another delivery to the station to occur before venting could onset. In general, for an underutilized station venting, and therefore, emission amounts were reasoned to be highly depended on local vehicle traffic. In certain cases, manual vents were performed by station operators to keep the station pressure low to benefit the vehicle fleet. Obviously, emissions caused from these practices could be reduced expediently and do not represent a deficiency in the technology since the vehicles can operate on the warmer LNG.

The first sensitivity analysis performed on segment 1 of audit 1 revealed the variation of ambient temperature to be the greatest parameter affecting pressure rise of the variables investigated. The first analysis modified the variables by $+/-10 \%$ and $+/-20 \%$. Dispense piping lengths was shown to be the second most sensitive parameter. However, after analyzing the changes which were likely in the station and not based on percentages but probable parameter variations the results altered. The assumed multilayer insulation thermal conductivity was found to be the strongest parameter. While a decrease in the thermal conductivity of the MLI did not affect pressure rise greatly a realistic increase resulted in a large change in the pressure rise. Ambient temperature had a reduced effect and reflected a more realistic uncertainty in the inputs. Since piping lengths were provided for the first audit station the original $10 \%$ and $20 \%$ variations were considered possible but were more indicative of variations expected in the second audit data. Overall the sensitivity analysis highlighted the importance of accurate values required in the station model as in any model.

\subsection{Recommendations}

Additional research needs to be carried out to obtain accurate fleet distributions among existing and future LNG stations. Accurate distributions for the fleets will enable an increased predictive power for LNG emissions by identifying the population of stations at risk for venting. Also, a more composite analysis involving multiple station configurations can be performed to increase the accuracy of emission predictions. In addition, a more accurate venting model for station PRV behavior should be developed if a similar approach as outline in this paper is used to extrapolate methane emissions.

Multiple strategies exist and can be utilized for the reduction of emissions from underutilized stations. Active pressure rise mitigation strategies can be installed on low throughput stations. These mitigation strategies include active vapor withdrawal for use in natural gas generators, and a refrigeration 
loop utilizing liquefied nitrogen among other methods. In addition, low throughput stations may benefit from flaring off returned vehicle methane vapor until throughput is high enough to eliminate PRV vents.

\subsection{Final Concluding Remarks}

All the research objectives for the project were met with varying degrees of success. The stratified thermodynamic and heat transfer approach was found to be necessary for the prediction of pressure rise in a stationary cryogenic tank with no fluid transfer. Both thermodynamic approaches performed well for an operating station model but the homogeneous model was more resilient. The homogeneous approach is recommended for future LNG station investigations of this type. Emission rates calculated for LNG stations represent a best estimate but further refinements are necessary to accurately characterize emission from LNG stations. Direct measurements of the PRV venting and characterization of the fleet traffic rates will be necessary in the future to better quantify methane emissions from LNG stations. Overall current emissions from LNG stations are low since there are less than 120 public stations in operation. The venting rate from stations can be significant but an overall inventory estimation would be complicated by super emitter issues; many stations will have zero vent emissions while others may vent significantly. In addition, the population of stations using specific technologies will need to be known to make any accurate estimations. For example some stations use liquid nitrogen to control the pressure and normally have no PRV emissions. Vent emission will only become a concern if transportation vehicles switch over to LNG in large quantities, requiring larger numbers of stations. In general, the more vehicles in the vehicle fleet the less likely stations will have PRV vent emissions. Likely, emissions will occur in the period when stations are being built to facilitate growing demand for LNG vehicles but when fleet numbers are not yet high with emissions trending toward zero (from the PRV) once operational vehicle numbers catch up. 


\section{References}

[1] R. A. Alvarez, S. W. Pacala, J. J. Winebrake, W. L. Chameides, and S. P. Hamburg, "Greater Focus Needed on Methane Leakage from Natural Gas Infrastructure," PNAS, vol. 109, pp. 64356440, 2012.

[2] S. Hamburg and R. Alvarez. What Will it Take to Get Sustained Benefits From Natural Gas? Available: https://www.edf.org/energy/methaneleakage

[3] N. Clark, D. McKain, W. S. Wayne, H. Li, D. Johnson, V. Akkerman, C. Sandeval, A. Covington, R. A. Mongold, J. Hailer, and O. Ugarte, "Development of a Methane Emissions Estimation Model for the Heavy-Duty Transportation Sector," Environmental Science \& Technology, Expected Publication May 2016.

[4] C. Sandoval, "Thermodynamics and Emission Modeling of Liquefied Natural Gas (LNG) Tanks and Fueling Stations," Ph.D., College of Mechanical and Aerospace Engineering, West Virginia University, 2015.

[5] R. F. Barron, Cryogenic Heat Transfer. New York: Taylor \& Francis, 1999.

[6] M. M. Foss, "Introduction to LNG," The University of Texas at Austin, Austin 2012.

[7] K. L. Cashdollar, I. A. Ziochower, G. M. Green, R. A. Thomas, and M. Hertzberg, "Flammability of Methane, Propane, and Hydrogen Gases," Journal of Loss Prevention in the Process Industries, 2000.

[8] C. A. Powars, "Best Practices to Avoid LNG Fueling Station Venting Losses," St. Croix Research 2010.

[9] (June, 2012). Portugal: Sines Gets LNG Cargo. Available: http://www.lngworldnews.com/portugal-sines-gets-lng-cargo-2/

[10] (August, 2014). APLNG Completes LNG Storage Tanks Testing. Available: http://www.lngworldnews.com/aplng-completes-lng-storage-tanks-testing/

[11] Taylor-Wharton. (2015). CryoLNG Products. Available: http://www.taylorwharton.com/default.aspx?pageid=687

[12] Chart Industries. (2015). LNG Solutions and Equipment - Fueling. Available: http://www.chartindustries.com/Energy/Energy-Products/LNG-Solutions-Equipment/Fueling

[13] R. F. Barron, Cryogenic Systems, Second ed. New York: Oxford University Press, 1985.

[14] C. Emmer, "A Short Primer on Cryogenic Tanks," in The Society of Naval Architects and Marine Engineers Great Lakes and Great Rivers Section, 2012.

[15] J. E. O'Brien and A. Siahpush, "Investigation of Low-Cost LNG Vehicle Fuel Tank Concepts," Idaho National Engineering and Evironmental Laboratory,1998.

[16] A. Hofmann, "The Thermal Conductivity of Cryogenic Insulation Materials and Its Temperatrue Dependence," Cryogenics, pp. 815-824, 2006.

[17] W. L. Johnson, "Thermal Performance of Cryogenic Multilayer Insulation at Various Layer Spacings," M.S. Aerospace Engineering, Mechanical, Materials, and Aerospace Engineering, University of Central Florida, Orlando, Florida, 2010.

[18] NIST. (2015). Material Properties: 304 Stainless (UNS S30400). Available: http://cryogenics.nist.gov/MPropsMAY/304Stainless/304Stainless_rev.htm

[19] A. Z. Sahin, "Effective Thermal Conductivity of Frost During the Crystal Growth Period," International Journal of Heat and Mass Transfer, vol. 43, 2000.

[20] D. A. Bahadori, Thermal Insulation Handbook for the Oil, Gas, and Petrochemical Industries: Gulf Professional Publishing, 2014.

[21] A. Demharter, "Polyurethane Rigid Foam, a Proven Thermal Insulating Material for Application Between +130 C and -196 C," Cryogenics, vol. 38, 1998. 
[22] X. B. Zhang, L. Yao, L. M. Qiu, Z. H. Gan, R. P. Yang, X. J. Ma, and Z. H. Liu, "Experimental Study on Cryogenic Moisture Uptake in Polyurethane Foam Insulation Material," Cryogenics, vol. 52, 2012.

[23] S. A. Dye, P. N. Tyler, G. L. Mills, and A. B. Kopelove, "Wrapped Multilayer Insulation Design and Testing," Cryogenics, vol. 64, 2014.

[24] D. Sheats and M. Capers, "Density Stratification in LNG Storage," Cold Facts, vol. 15, 1999.

[25] L. B. Evans, R. C. Reid, and E. M. Drake, "Transient Natural Convection in a Vertical Cylinder," AlChE Journal, vol. 14, 1968.

[26] J. W. Sheffield, S. Gursu, S. A. Sherif, and T. N. Veziroglu, "Analysis and Optimization of Thermal Stratification and Self-Pressurization Effects in Liquid Hydrogen Storage Systems -Part 1: Model Development," Journal of Energy Resources Technology, vol. 115, 1993.

[27] M. Seo and S. Jeong, "Analysis of Self-Pressurization Phenomenon of Cryogenic Fluid Storage Tank With Thermal Diffusion Model," Cryogenics, pp. 549-555, 2009.

[28] Y. Li, R. Wang, and C. Wang, "Study on Effects of Liquid Level on the Heat Leak into Vertical Cryogenic Vessels," Cryogenics, vol. 50, 2010.

[29] S. Bates and D. S. Morrison, "Modelling the Behaviour of Stratified Liquid Natural Gas in Storage Tanks: a Study of the Rollover Phenomenon," Elsevier Science Ltd, vol. 40, pp. 18751884, 1997.

[30] K. B. Deshpande, W. B. Zimmerman, M. T. Tennant, M. B. Webster, and M. W. Lukaszewski, "Optimization Methods for The Real-Time Inverse Problem Posed by Modelling of Liquefied Natural Gas Storage," Chemical Engineering Journal, vol. 170, 2011.

[31] D. H. Riemer, "Cryogenic Tank Stratification a Simpler Approach," Advances in Cryogenic Engineering, 1986.

[32] C. H. Panzarella and M. Kassemi, "On the Validity of Purely Thermodynamic Descriptions of Two-Phase Cryogenic Fluid Storage," Journal of Fluid Mechanics, vol. 484, 2003.

[33] H. M. Jr., J. A. Clark, and H. Z. Barakat, "Finite Difference Soultion of Stratification and Pressure Rise in Containers " The University of Michigan, 1968

[34] R. W. Arnett and R. O. Voth, "A Computer Program for the Calculation of Thermal Stratification and Self-Pressurization in a Liquid Hydrogen Tank," NASA, Washington, D.C.1972.

[35] J. Ren, J. Shi, P. Liu, M. Bi, and K. Jia, "Simulation on Thermal Stratification and DeStratification in Liquefied Gas Tanks," International Journal of Hydrogen Energy, vol. 38, 2013.

[36] J. Shi, J. Ren, P. Liu, and M. Bi, "Experimental Research on the Effects of Fluid and Heater on Thermal Stratification of Liquefied Gas," Experimental Thermal and Fluid Science, vol. 50, 2013.

[37] S. Barsi and M. Kassemi, "Numerical and Experimental Comparisons of the Self-Pressurization Behavior of an LH2 Tank in Normal Gravity," Cryogenics, vol. 48, 2008.

[38] M. Gandhi, J. Joshi, and P. Vijayan, "Study of Two Phase Thermal Stratification in Cylinderical Vessels: CFD Simulations and PIV Measurements," Chemical Engineering Science, vol. 98, 2013.

[39] S. Anghaie and Z. Ding, "Modeling of Bulk Evaporation and Condensation," University of Florida, Gainesville, Florida, 1996.

[40] Q. S. Chen, J. Wegrzyn, and V. Prasad, "Analysis of Temperature and Pressure Changes in Liquefied Natural Gas (LNG) Cryogenic Tanks," Cryogenics, vol. 44, pp. 701-709, 2004.

[41] C. Chung, S. Jeon, B. Jin, and Y. Kim, "Consistent Thermal Analysis Procedure of LNG Storage Tank," Structural Engineering and Mechanics, vol. 25, pp. 445-466, 2006.

[42] E. Adom, S. Z. Islam, and X. Ji, "Modelling of Boil-Off Gas in LNG Tanks: A Case Study," International Journal of Engineering and Technology, vol. 2, pp. 292-296, 2010.

[43] C. Park, S. Hong, and S. Kim, "Model Predictions for Efficiency Operation of the LNG Storage Tank," presented at the 22nd World Gas Conference, Tokyo, 2003.

[44] A. Hofmann, "Determination of The Pressurising Period for Stored Cryogenic Fluids," Cryogenics, vol. 46, pp. 825-830, 2006. 
[45] R. S. R. Gorla, "Rapid Calculation Procedure to Determine the Pressurizing Period for Stored Cryogenic Fluids," Applied Thermal Engineering, vol. 30, 2010.

[46] C. Ludwig, M. E. Dreyer, and E. J. Hopfinger, "Pressure Variations in a Cryogenic Liquid Storage Tank Subjected to Periodic Excitations," International Journal of Heat and Mass Transfer, vol. 66, 2013.

[47] T. L. Bergman, A. S. Lavine, F. P. Incropera, and D. P. Dewitt, Introduction to Heat Transfer. Hoboken, NJ: John Wiley \& Sons, Inc., 2011.

[48] Y. A. Cengel, Heat Transfer: A Practical Approach. New York, NY: Mcgraw-Hill, 2002.

[49] Department of Energy. National Solar Radiation Data Base. Available: http://rredc.nrel.gov/solar/old_data/nsrdb/

[50] Chart Industries. (2014). MVIP Python: Modular Vacuum Insulated Pipe [Technical Specifications]. Available: http://files.chartindustries.com/11030862_2014_MVIPpython2.pdf

[51] R. D. Clear, L. Gartland, and F. C. Winkelmann, "An Empirical Correlation for Outside Convective Air-Film Coefficient for Horizontal Roofs," Energy and Buildings, vol. 35, pp. 797811, 2003.

[52] Chart Industries. (2015). Data Book: for Cryogenic Gases and Equipment. Available: http://files.chartindustries.com/10517513.RevM.Databook.2015.pdf

[53] S. Fall, A. Watts, J. Nielsen-Gammon, D. N. Evan Jones, J. R. Christy, and R. A. Pielke, "Analysis of the Impacts of Station Exposure on the U.S. Historical Climatology Network Temperatures and Temperature Trends," Journal of Geophysical Research, vol. 116, 2011.

[54] A. Watts, "Is the U.S. Surface Temperature Record Reliable," The Heartland Institute, Chicago, Illinios 2009.

[55] Enraf, "The Art of Tank Gauging," Delft Instruments, Netherlands.

[56] F. J. Berto, "Hydrostatic Tank Gauges Accurately Measure Mass, Volume, and Level," Oil and Gas Journal, vol. 80, 1990.

[57] "Cryostar Accurately Measures LNG Fuel with Micro Motion Coriolis Meters," Emerson Process Management, 2011.

[58] G. W. Burns and M. G. Scroger, "Nist Measurement Services: The Calibration of Thermocouples and Thermocouple Materials," NIST NIST Special Publication 250-35, 1989.

[59] J. D. Wright, J.-P. Kayl, A. N. Johnson, and G. M. Kline, "Gas Flowmeter Calibrations with the Working Gas Flow Standard," NIST Special Publication 250-80, 2009.

[60] "Ultra-Portable Greenhouse Gas Analyzer User Manual," Los Gatos Research, 2014. 


\section{A-Appendix: Additional Model Development Information}

\section{A-1 Horizontal Tank Equations}

This section details the necessary modifications of the vertical tank heat transfer formulation to a horizontal tank. The horizontal tank formulation was not implemented but was included for completeness. In the homogeneous case the heat transfer model for the two orientations was extremely similar. Some correlations for external convection were different but overall convection was negligible due to the large resistances in the tank wall; therefore, the vertical tank formulation was considered a good approximation and always used. The stratified thermodynamics was developed only for a vertical tank thus only the vertical stratified tank heat transfer model was utilized.

Equations for the conduction through the horizontal tank were the same as shown in the vertical tank development. Convection correlations were handled similarly but applied to different portions of the tank. The tank end regions were handled with the same approach as detailed for Equations $22-25$. The convection equations for the tank ends use equation 38 for the natural convection term in Equations 22 25.

$$
\overline{N u}_{L}=\left\{0.825+\frac{0.387 R a_{L}^{1 / 6}}{\left[1+(.492 / P r)^{9 / 16}\right]^{8 / 27}}\right\}^{2}
$$

Natural convection of the cylindrical portion of the tank was calculated by Equation 39. The equation for forced convection stayed the same.

$$
\overline{N u}_{D}=\left\{0.60+\frac{.387 R a_{D}^{1 / 6}}{\left[1+(.559 / P r)^{9 / 16}\right]^{8 / 27}}\right\}^{2} \quad R a_{D} \leq 10^{12}
$$

Equations and considerations for thermal stratification were formulate in the same way but utilized different equations. Unlike in the vertical tank the surface area each layer was exposed to was different. Calculating the varying wetted surface areas was the major difference between the two formulations. Figure A-1 shows an illustration of the horizontal tank layer spacing on left, and geometry for calculating the varying surface areas on the right.

As in the vertical tank the layers were divided by equal height amounts based on the number layers desired in the tank heat transfer calculation. First the area exposed to the liquid was solved for by using the volume of liquid inside the tank. The volume inside the tank determined the height of liquid and was related to the surface area at the ends of the tank as shown in Figure A-1. 


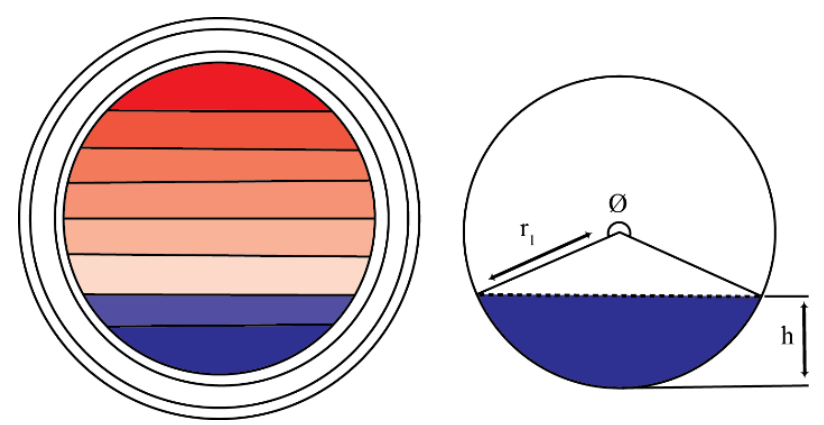

Figure A-1: Horizontal Tank Stratification Layers and Geometry

The blue area was the area wetted by liquid level. For the study in many cases only the volume or mass of LNG was known. The wetted area was related to the volume of liquid in the tank by $A * L=$ volume since the length and volume were known. The calculated area was then used in equation 40 .

$$
A=\frac{R^{2}}{2}(\varnothing-\sin \varnothing)
$$

The angle Phi found in Figure A-1 was solved for by iteration in equation 40. Once the angle was found equation 41 was used to find the height of liquid inside the tank.

$$
h=R(1-\cos \emptyset / 2)
$$

The height was then subdivided into equal height layers just as with the vertical tank. The area of the layer exposed to the cylindrical surface of the tank was calculated by first using equation 42 to calculate the angle at the top and bottom of the layer.

$$
\varnothing=2 \operatorname{Cos}^{-1}\left(\frac{R-h}{R}\right)
$$

Equation 44 was then used to find surface area for each angle. The two surface areas were then subtracted to find the surface area along cylindrical portions of the tank.

$$
A_{s, c y l}=\emptyset R L
$$

The tank ends were handled in the same way utilizing equation 40 instead. 


\section{A-2 Station Tank Area Ratio Calculation}

The calculation of the area ratio parameter is extremely important to the analysis due to the disparity between the thermal conductivities of the strut material and insulation. A straight forward process is to use the design acceleration loading of the vessel to calculate the area required to statically support that load. The size of the tank and capacity can be found from manufacturer specifications and then be use for the AR calculation in conjunction with the table below. Table A-1 [2] gives some example design load for the strut support system found in the annulus of a cryogenic tank.

Table A-1: Acclerations Loads for Cryogenic Fluid Storage Vessel Suspension System

\begin{tabular}{|l|l|l|l|l|}
\hline \multicolumn{1}{|c|}{ Type of unit } & \multicolumn{1}{|c|}{$\begin{array}{c}\text { Vertical Up } \\
\text { (g) }\end{array}$} & $\begin{array}{c}\text { Vertical Down } \\
\text { (g) }\end{array}$ & $\begin{array}{c}\text { Transverse } \\
\text { (g) }\end{array}$ & $\begin{array}{c}\text { Longitudinal } \\
\text { (g) }\end{array}$ \\
\hline Stationary Vessels: & & & & \\
\hline Empty & 0.5 & 3 & 0.5 & 0.5 \\
\hline Full & 0.5 & 1.5 & .5 & 0.5 \\
\hline Full with blast loading & 3 & 5 & 4 & 4 \\
\hline Transport trailers: & & & & \\
\hline Small $\left(<4 \mathrm{~m}^{3}\right)$ & 2 & 5 & 4 & 8 \\
\hline Large $\left(>8 \mathrm{~m}^{3}\right)$ & 1 & 4 & 2 & 4 \\
\hline
\end{tabular}

Using the design accelerations the cross sectional area of stainless steel required to hold the full tank weight can be calculated. Once this value is known dimensions of the tank are used to calculate the surface area of the tank. The cross sectional area of stainless steel support materials divided by the surface area of the tank results in an estimate for the area ratio parameter. 


\section{A-3 Mean Thermal Conductivity}

Thermal conductivity is not constant and when high temperature disparities are involved changes in thermal conductivity cannot be ignored. One approach available is calculation using the mean thermal conductivity as outlined in [13]. Equation 44 is used to calculate the mean thermal conductivity in a material.

$$
k_{m}=\left(K_{h}-K_{c}\right) /\left(T_{h}-T_{c}\right)
$$

The temperature of the hot end of the material is $T_{h}$ and the temperature of the cold end is $T_{c}$. The variables $\mathrm{K}_{\mathrm{h}}$ and $\mathrm{K}_{\mathrm{c}}$ are the integral of the thermal conductivity function for the specific material at the hot and cold temperatures, respectively. Equation 45 is used to calculate the integral thermal conductivity. The lower bound of the integral can be set lower if data for the material exists at low a lower temperature.

$$
K=\int_{4 k}^{T} k_{t} d T
$$

If the function for the specific heat of the material is known this procedure may also be used to find the mean specific heat. 


\section{B-Appendix B: Additional Data Collection Information}

\section{B-1 Vehicle Tank Sensor Accuracy}

Figure B-1 and Figure B-2 show the experimental set up for the both the IFP tank and the NONIFP tank. Data was collected from the tanks for ambient temperature, surface temperatures, solar loading, weight, and pressure. Ambient temperature was collected by a k-type thermocouple. Surface temperatures were collected by two more k-type thermocouples. The thermocouples were mounted on the middle top (sunny) portion, and the middle bottom (shaded) portion of the tank. Each thermocouple was calibrated as detailed in [58]. The accuracy of each thermocouple was $2.2^{\circ} \mathrm{C}$. Solar loading was also collected on the sunny and shaded portions of the tank. Solar cells were calibrated to a Campbell Scientific LI-200X solar loading sensor. The calibration procedure exposed all sensors to solar loading conditions over a 24 hour period. The accuracy of the sensors were $+/-3 \%$. Tank weight was measured by two S-beam load cells each calibrated by test weights at WVU. The accuracy of the weighing system was $+/-.45 \mathrm{~kg}$. The pressure transducer used was a pre-calibrated cryogenic pressure sensor from Omega. The accuracy of the sensor was +/- 8.6 kPa. In addition, each tank was purged with ultra-high purity nitrogen before fueling. The manufactures recommended practices were followed for hot filling both tanks. Both tanks were filled at a Local LNG station providing saturated temperature fuel. Once filled the tanks were transported back to a CAFEE laboratory where they were set in the experimental apparatus. Data was logged by an in house data logging software called Scimitar. Table B-1 summarizes the sensors and associated sensor uncertainty.

Table B-1: Sensor Summary

\begin{tabular}{|l|l|l|}
\hline \multicolumn{1}{|c|}{ Independent Variable } & \multicolumn{1}{|c|}{ Measurement Type } & Uncertainty/Error \\
\hline Ambient Temperature & K-Type Thermocouple & $2.2^{\circ} \mathrm{C}$ \\
\hline LNG tank surface temperature (sunny)* & K-Type Thermocouple & $2.2^{\circ} \mathrm{C}$ \\
\hline LNG tank surface temperature (shaded)* & K-Type Thermocouple & $2.2^{\circ} \mathrm{C}$ \\
\hline Total solar loading (sunny)* & Campbell Scientific LI-200X & $+/-3 \%$ \\
\hline Total solar loading (shaded)* & WVU Solar Cell Calibrated to LI-200X & $+/-3 \%$ \\
\hline Tank Weight & S-Beam Load Cell & $+/-.45 \mathrm{~kg}$ \\
\hline Tank Pressure & Thin Film Cryogenic Pressure Transducer & $+/-8.6 \mathrm{kPa}$ \\
\hline Time & Data logging computer & -- \\
\hline
\end{tabular}




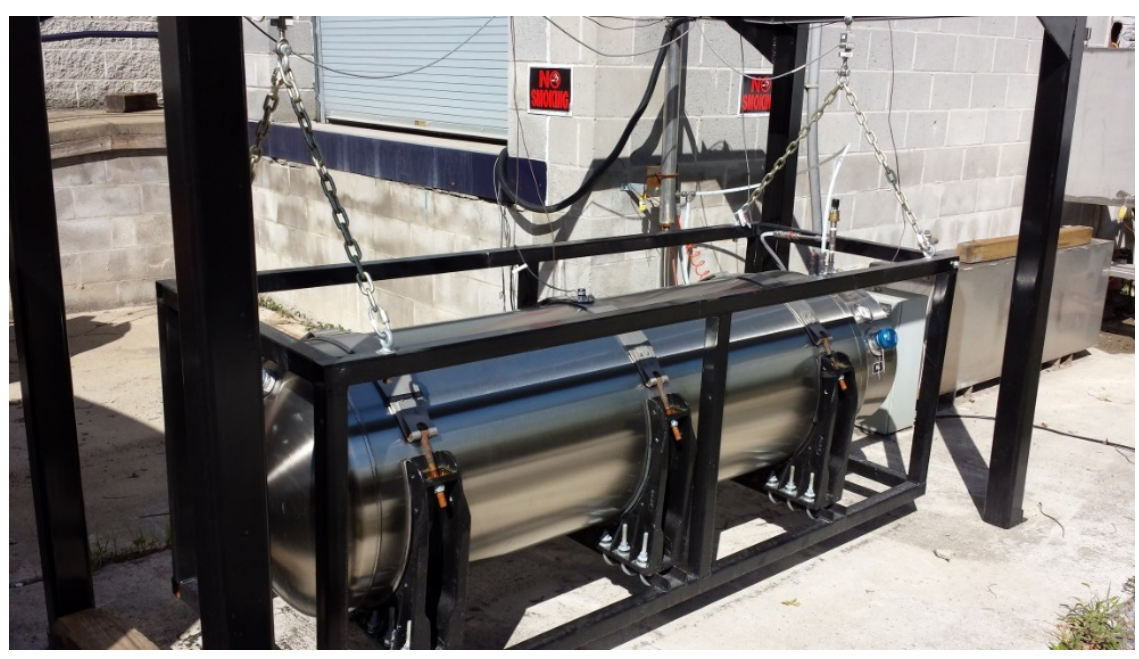

Figure B-1: NON-IFP LNG Tank Attached to Data Acquisition System

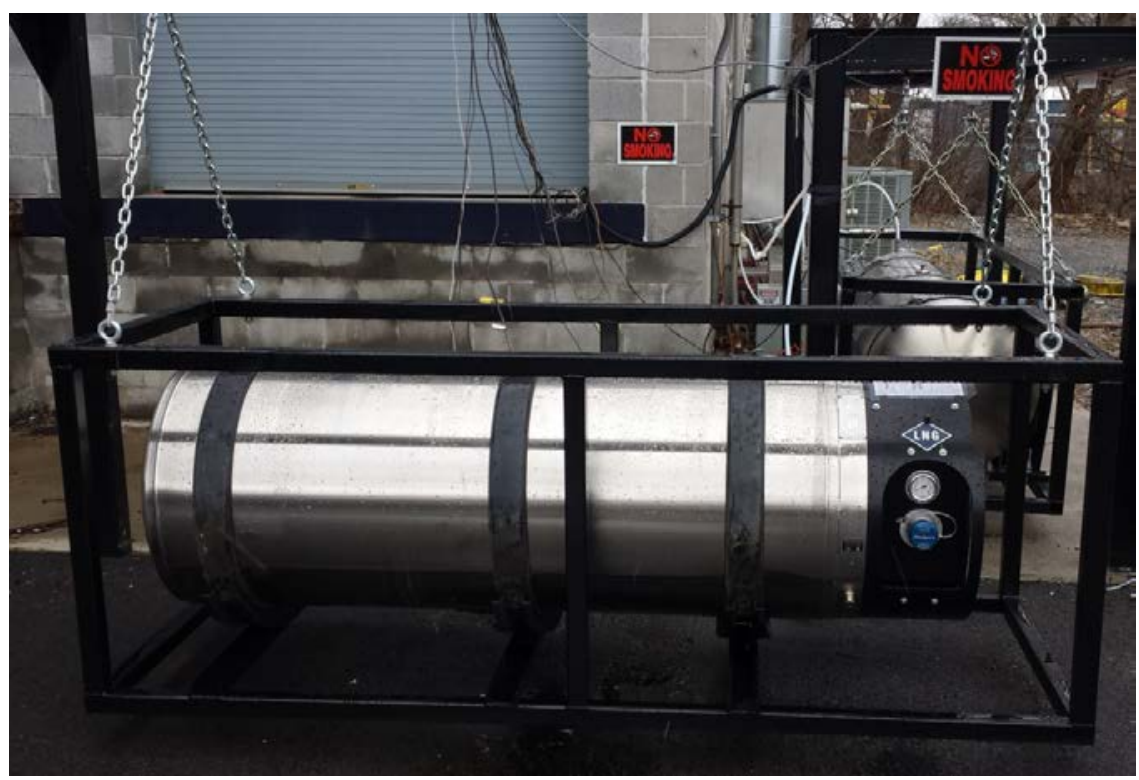

Figure B-2: IFP LNG Tank Attached to Data Acquisition System 


\section{B-2 Vehicle Tank NER Sensor Accuracy}

The normal evaporation experiments (NER) were conducted on the LNG vehicle tanks tested in the pressure rise experiments. Each tank was tested by opening the pressure release valve and allowing the dewar to vent to atmospheric pressure. The vented gas was directed through a flexible tube to a full flow sampling system (FFS). The FFS consisted of a hose which fed into an explosive-proof blower with the air exiting through a long straight tube. A mass airflow sensor (MAF) was installed prior to the exit tube and after the blower. Down steam in the exit tube the pipe was tapped were a LGR (Los Gatos Research) Ultraportable Greenhouse Gas Analyzer then withdrew a small portion of the flow to find the concentration of methane. The setup is shown Figure B-3.

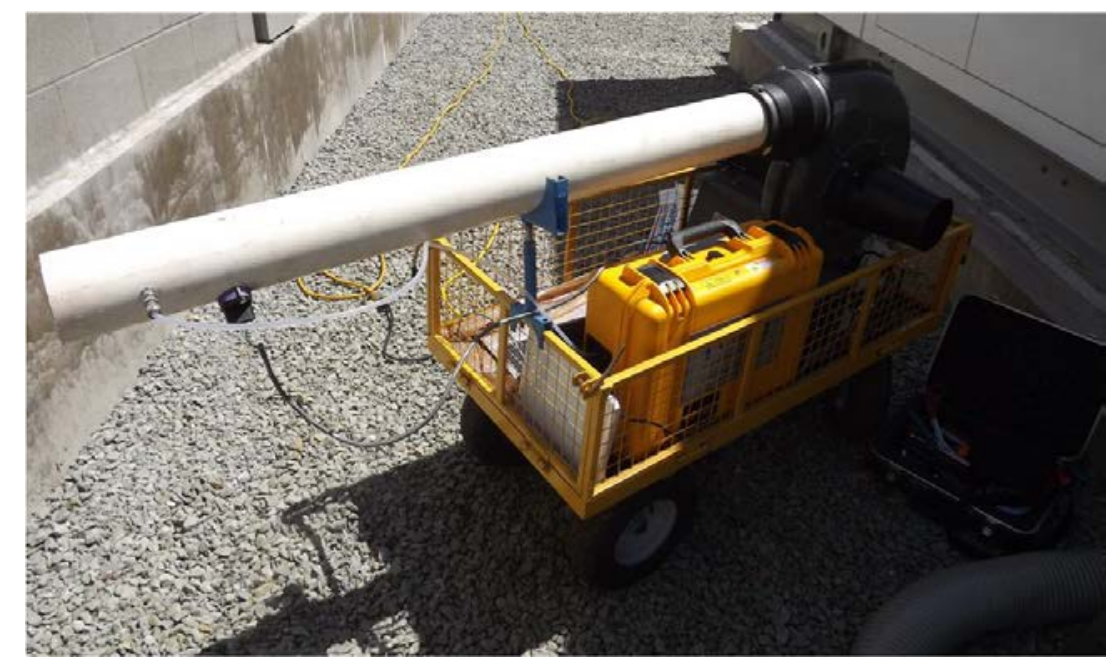

Figure B-3: Full Flow Sampling System

The MAF was an Abaco DBX $97 \mathrm{~mm}$. The MAF was capable of measuring flow rates up to 6800 $\mathrm{kg} / \mathrm{hr}$. Calibration of the MAF was completed using a 6 in. diameter LFE with model number: Z50MC2-6. The calibration procedure followed the standard guideline detailed in [59]. The uncertainty in the sensor was $4 \%$ ACFM.

The LGR sensor was capable of measuring methane, $\mathrm{CO}_{2}$, and water vapor. The sensor was calibrated to methane following the operation manual [60]. For calibration a specific concentration of methane was produced. A gas divider in conjunction with NIST traceable zero grade air and methane were used to produce the specific concentration. The sensor was accurate to within 1\% PPM. The FFS leak rate accuracy was $+/-4.4 \% \mathrm{~g} / \mathrm{hr}$. 


\section{C-Appendix: Simulation Result Plots}

\section{C-1 Hold Time Simulations}

NON-IFP Tank Exp. 2

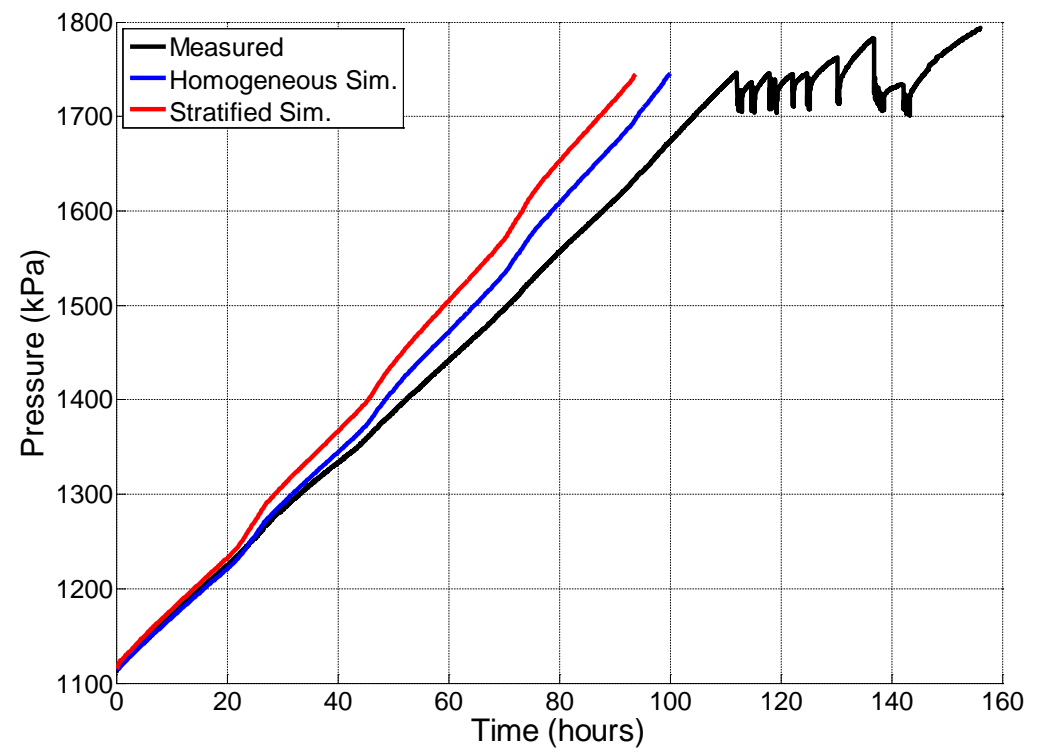

Figure C-1: NON-IFP Tank Exp. 2 Hold Time Simulation Pressure Comparison

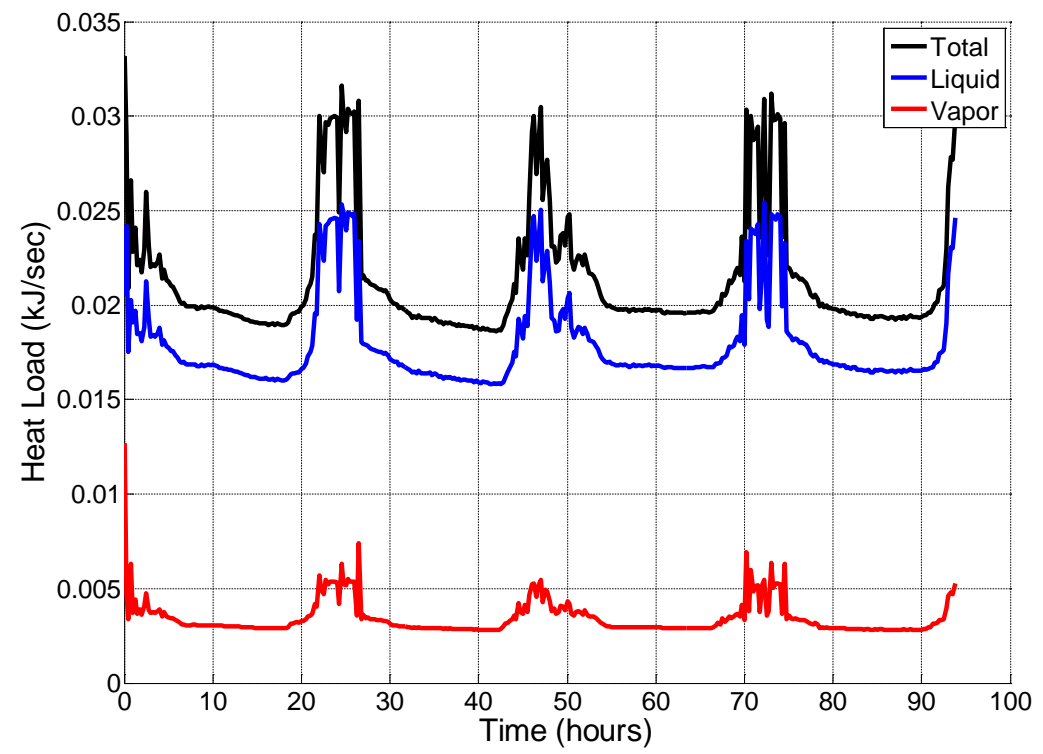

Figure C-2: NON-IFP Tank Exp. 2 Hold Time Simulation Stratified Tank Heat Transfer 


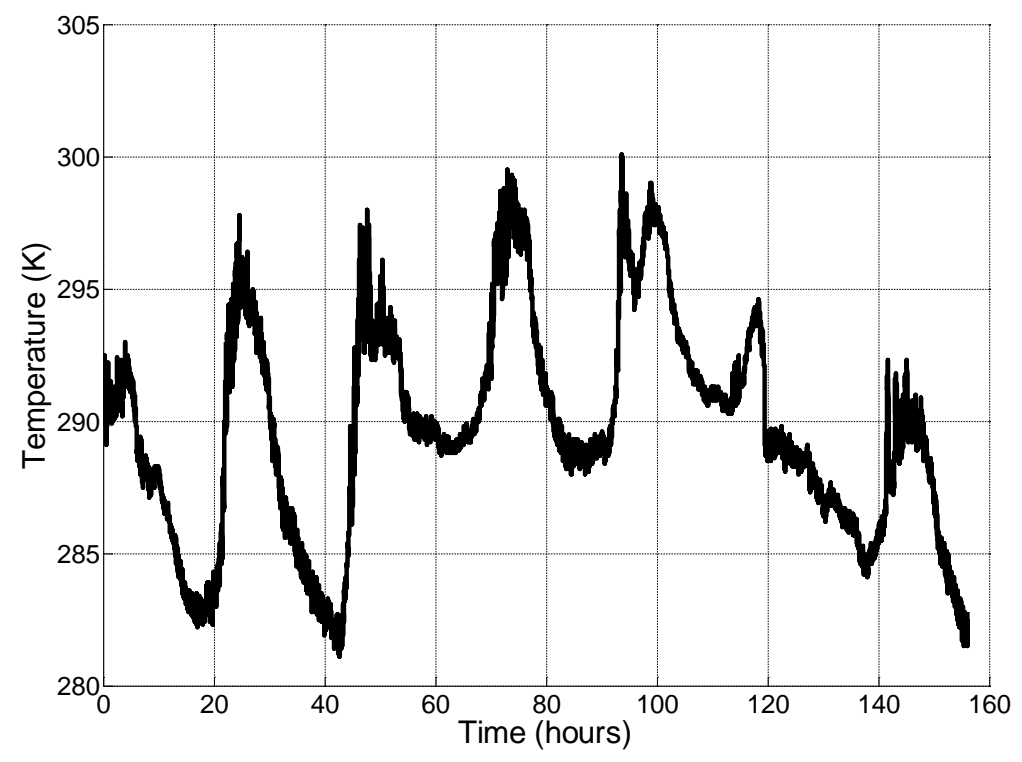

Figure C-3: NON-IFP Tank Exp. 2 Hold Time Simulation Ambient Temperature

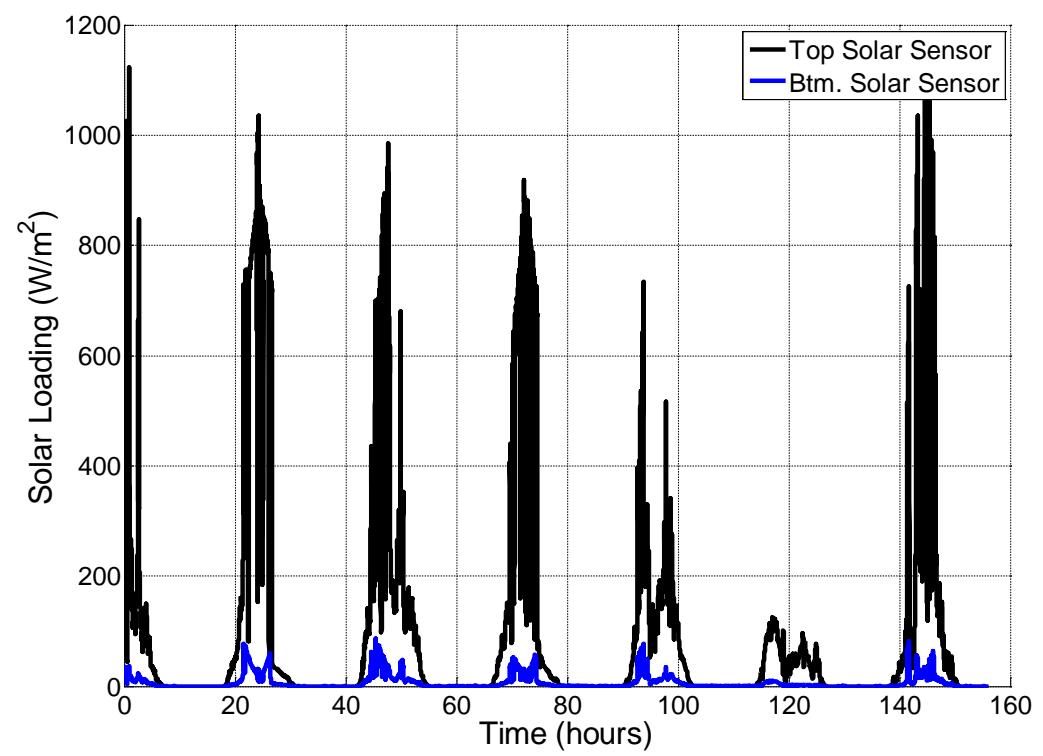

Figure C-4: NON-IFP Tank Exp. 2 Hold Time Simulation Solar Loading 


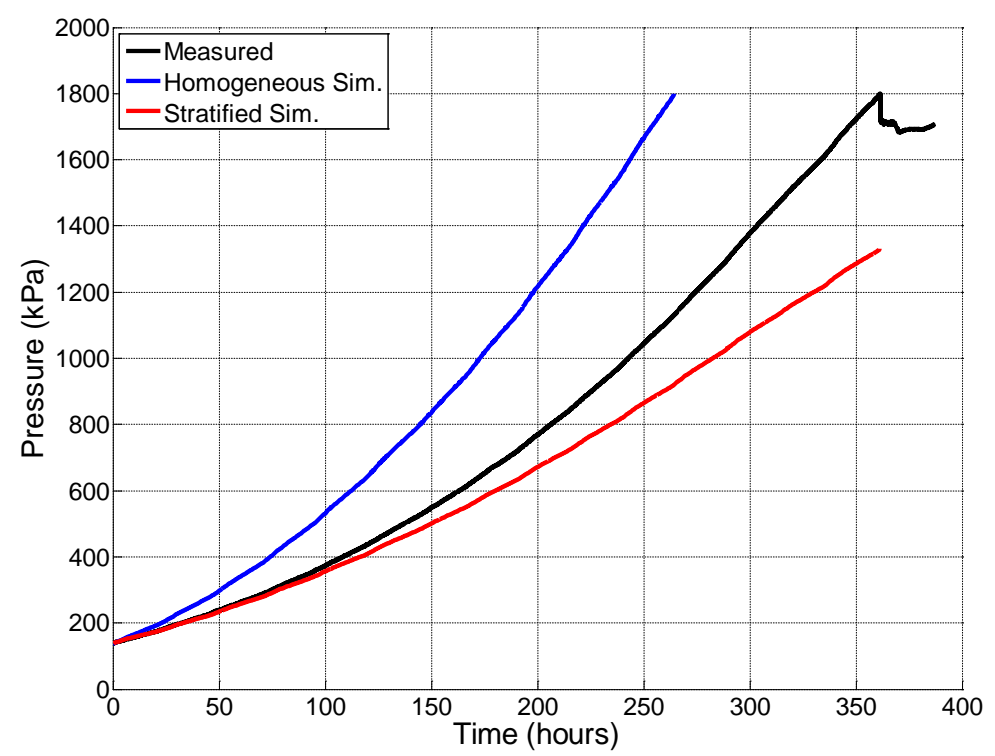

Figure C-5: NON-IFP Tank Exp. 3 Hold Time Simulation Pressure Comparison

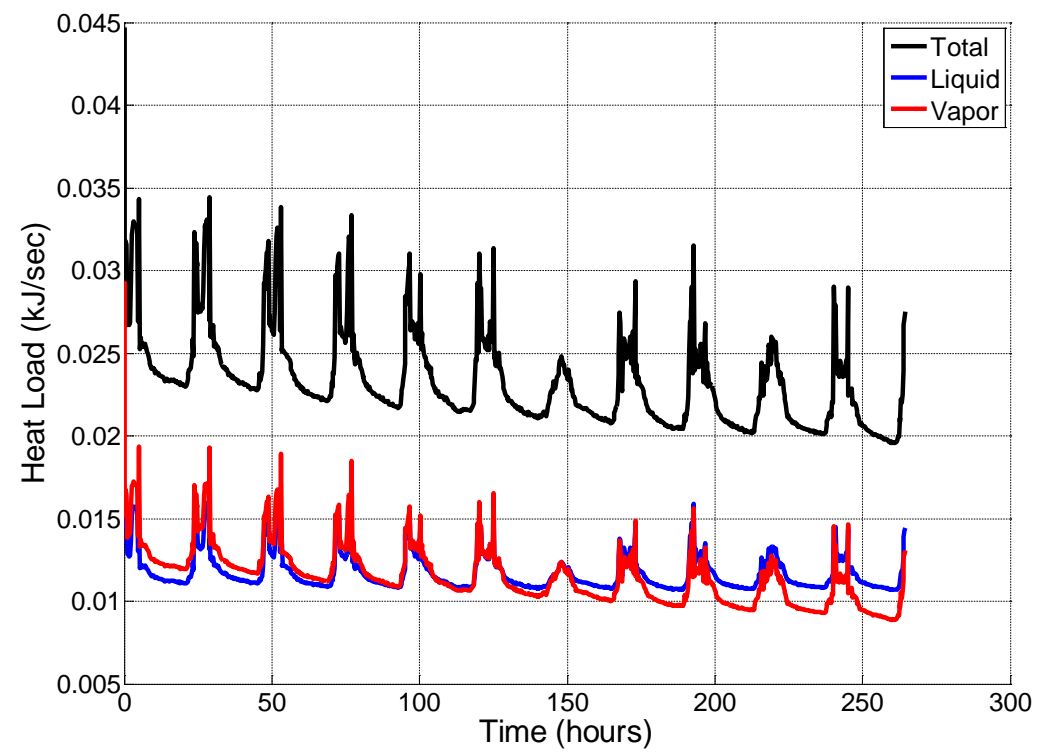

Figure C-6: NON-IFP Tank Exp. 3 Hold Time Simulation Stratified Tank Heat Transfer 


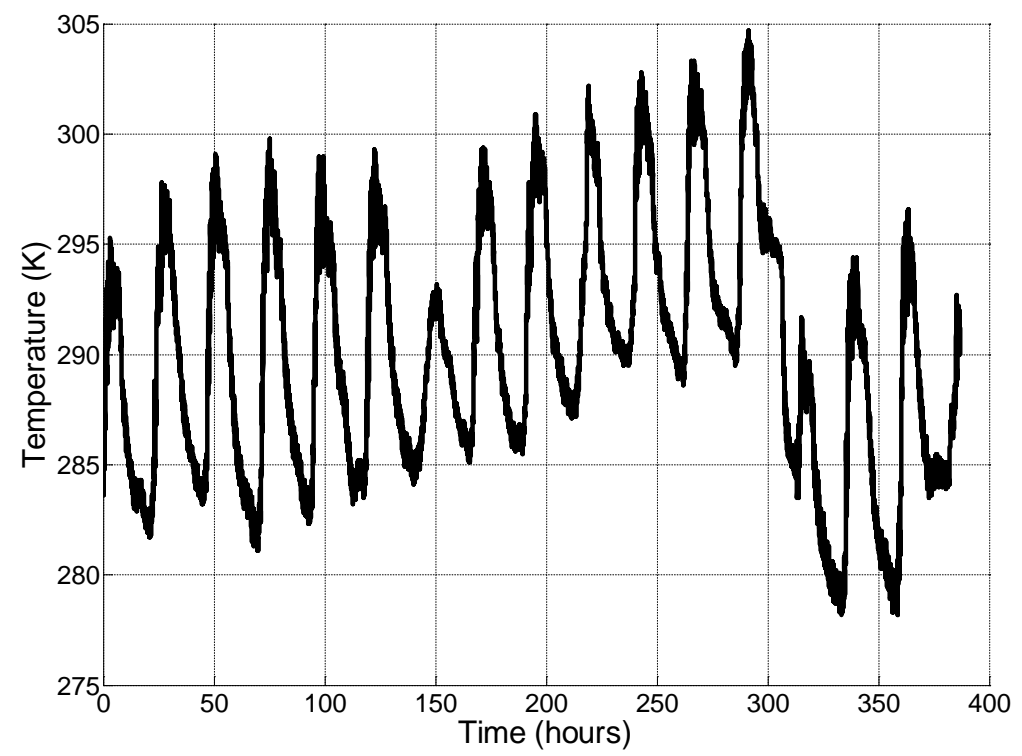

Figure C-7: NON-IFP Tank Exp. 3 Hold Time Simulation Ambient Temperature

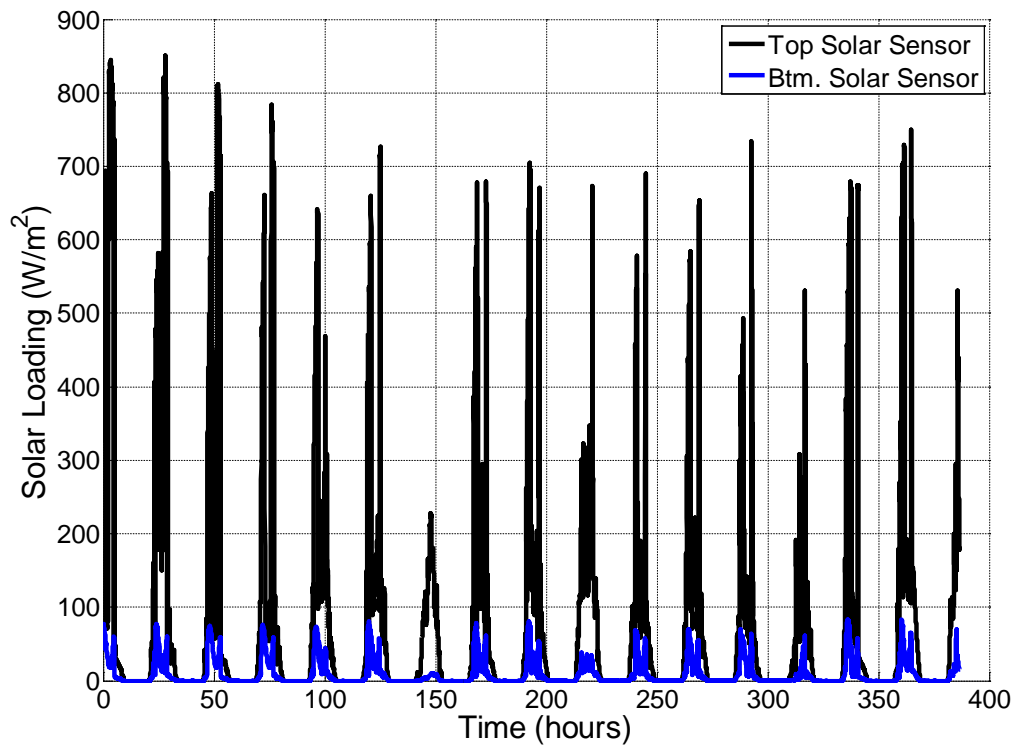

Figure C-8: NON-IFP Tank Exp. 3 Hold Time Simulation Solar Loading 


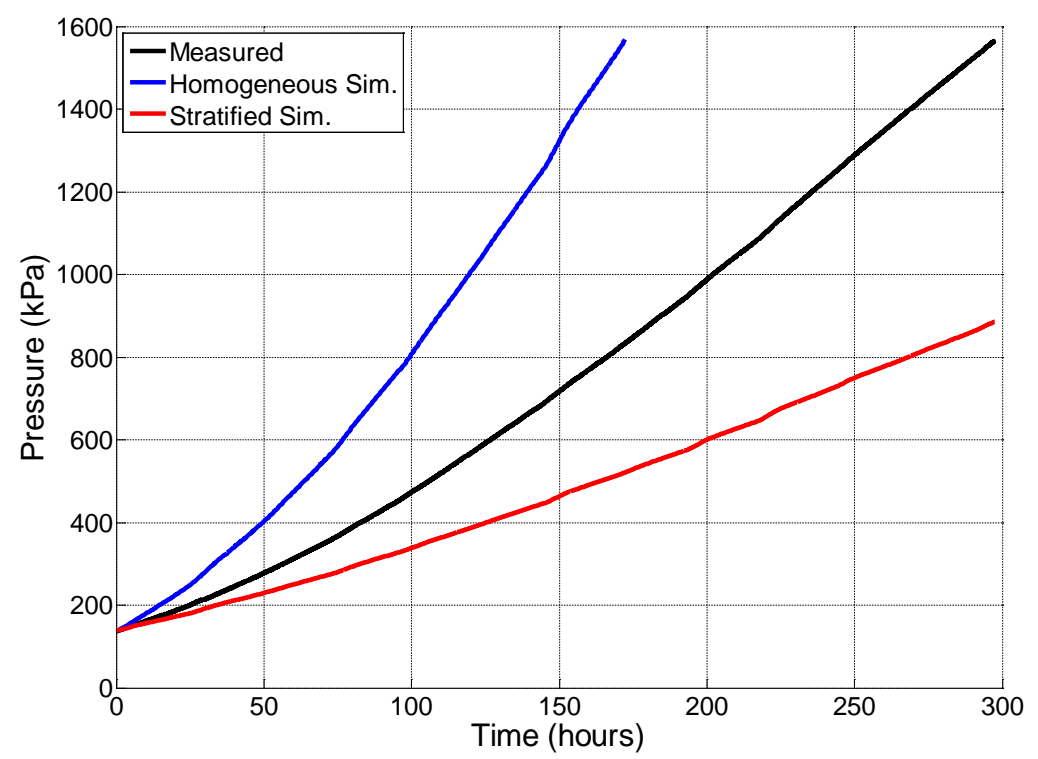

Figure C-9: NON-IFP Tank Exp. 4 Hold Time Simulation Pressure Comparison

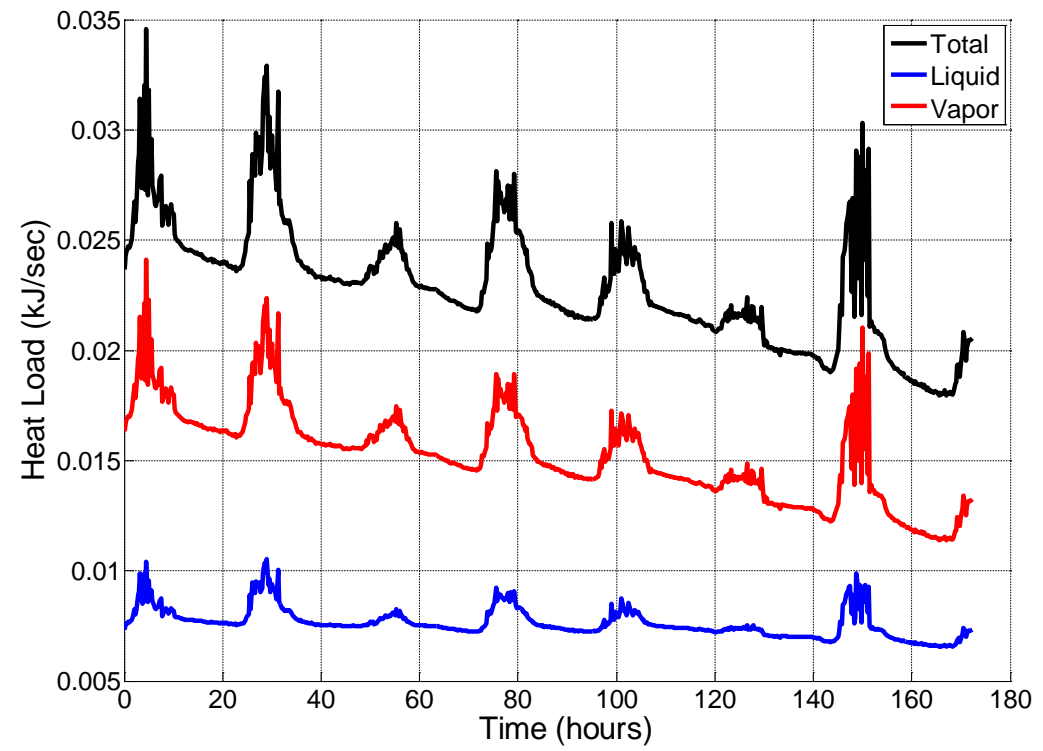

Figure C-10: NON-IFP Tank Exp. 4 Hold Time Simulation Stratified Tank Heat Transfer 


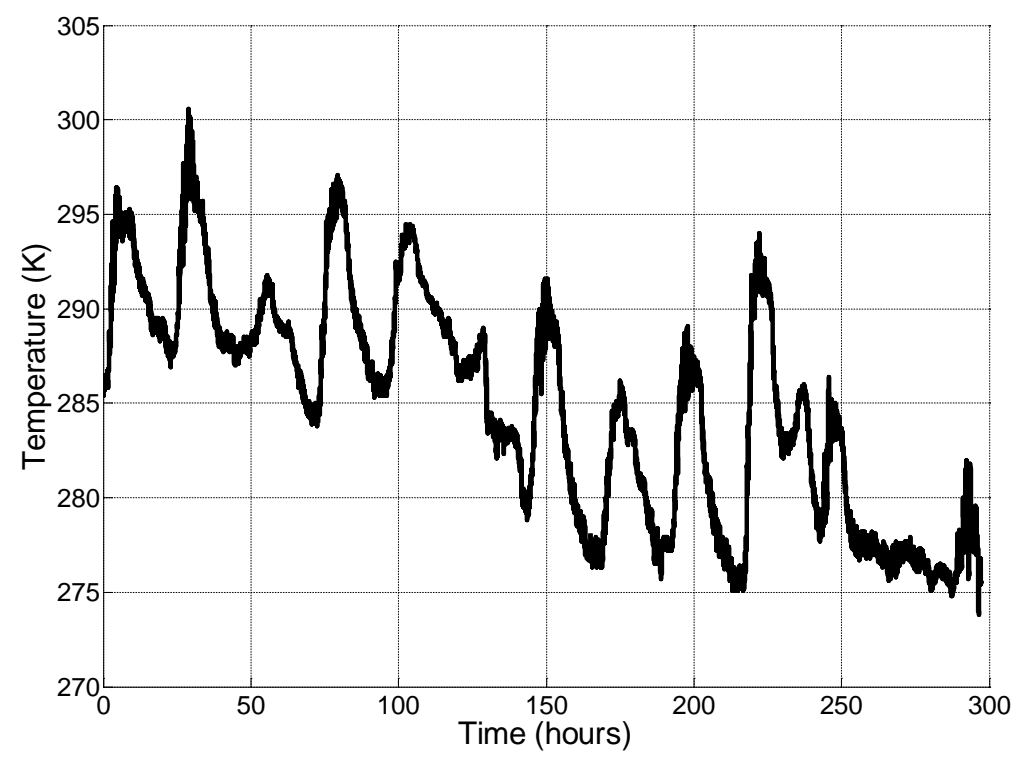

Figure C-11: NON-IFP Tank Exp. 4 Hold Time Simulation Ambient Temperature

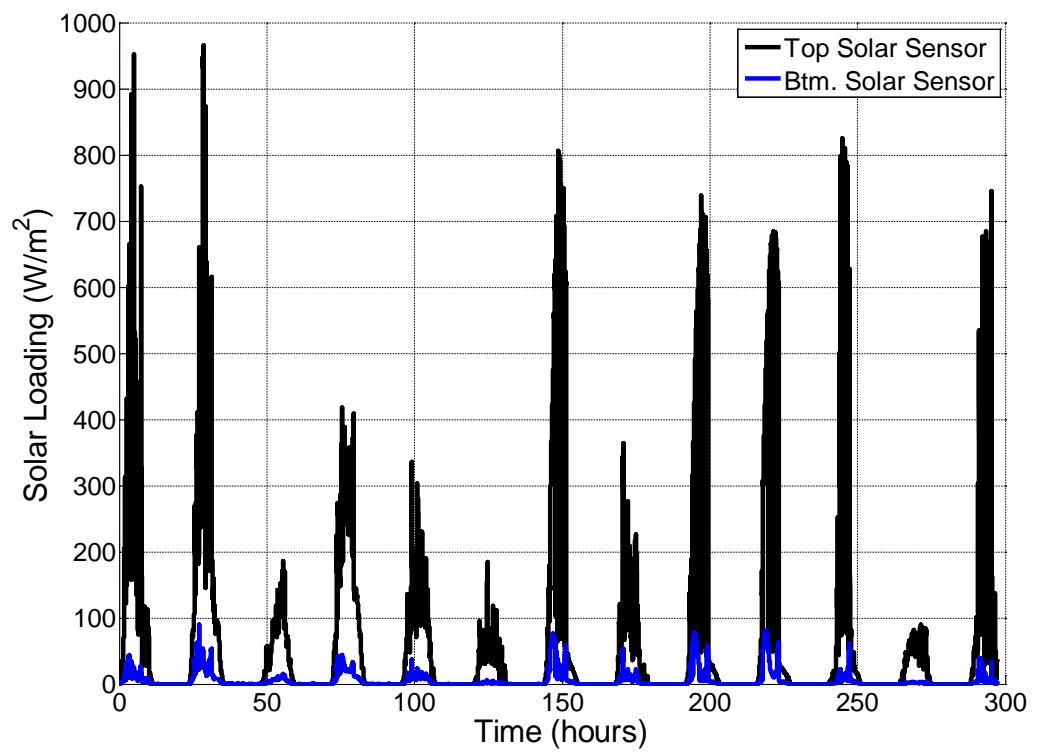

Figure C-12: NON-IFP Tank Exp. 4 Hold Time Simulation Solar Loading 


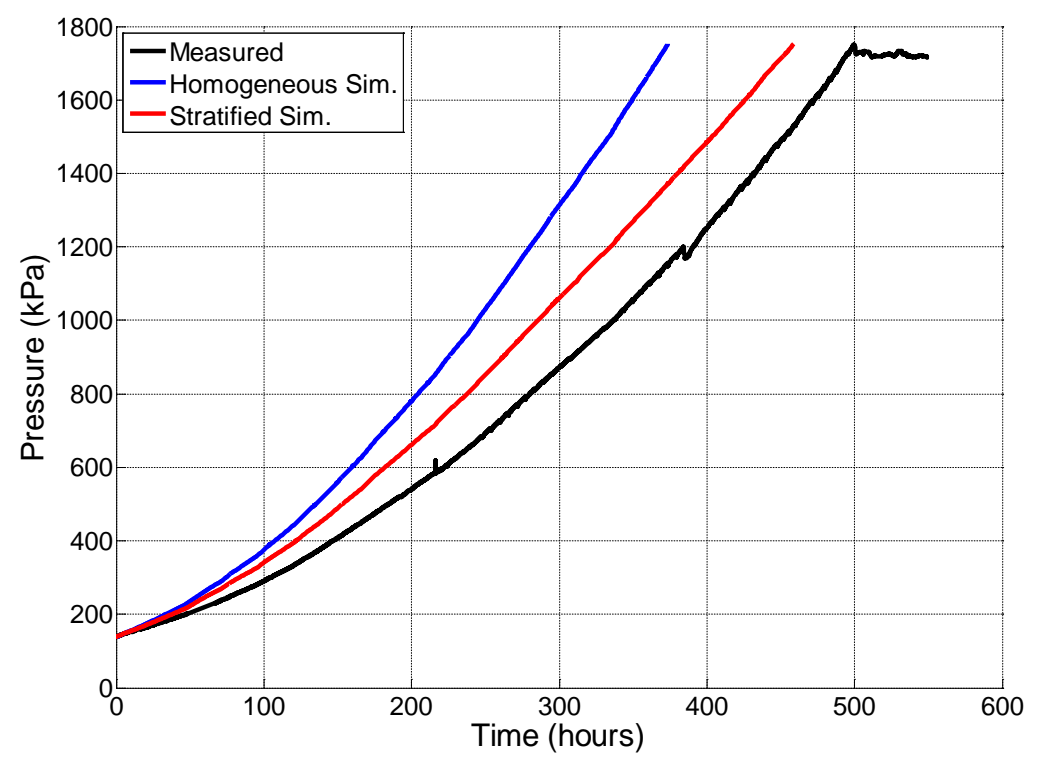

Figure C-13: NON-IFP Tank Exp. 5 Hold Time Simulation Pressure Comparison

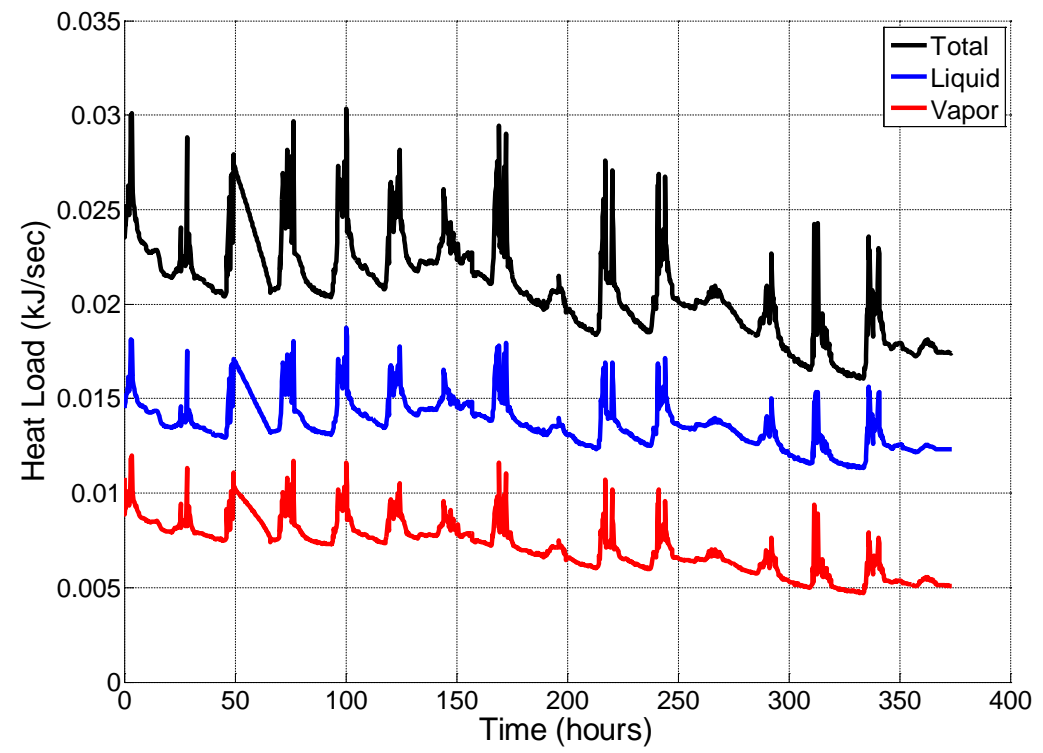

Figure C-14: NON-IFP Tank Exp. 5 Hold Time Simulation Stratified Tank Heat Transfer 


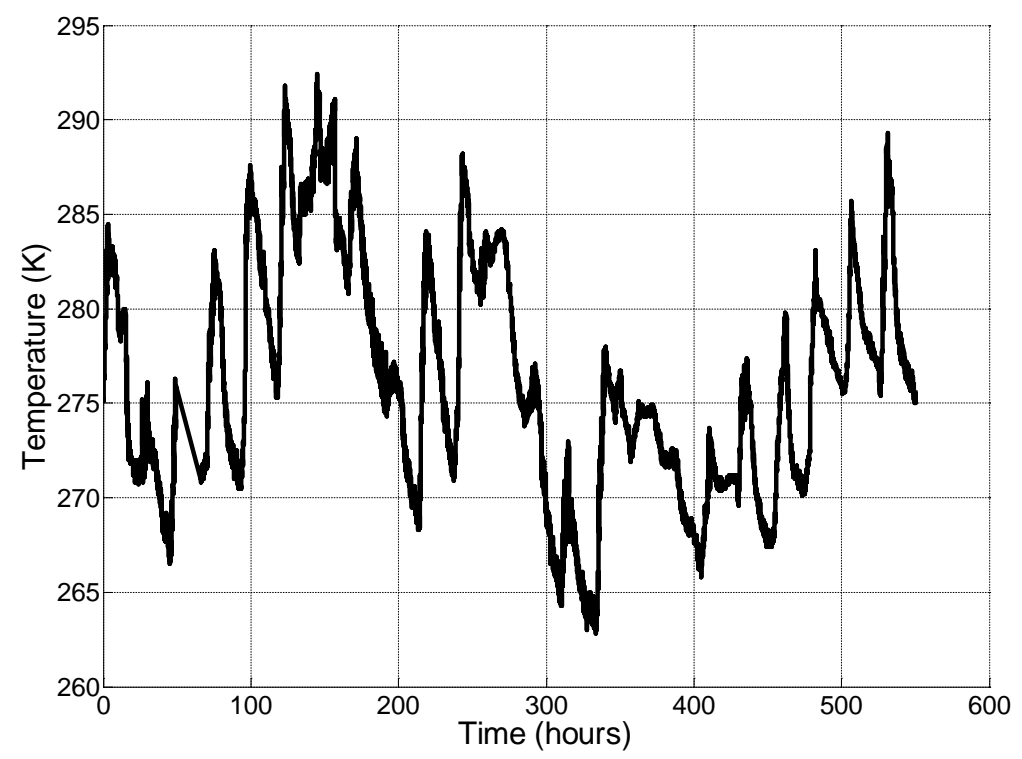

Figure C-15: NON-IFP Tank Exp. 5 Hold Time Simulation Ambient Temperature

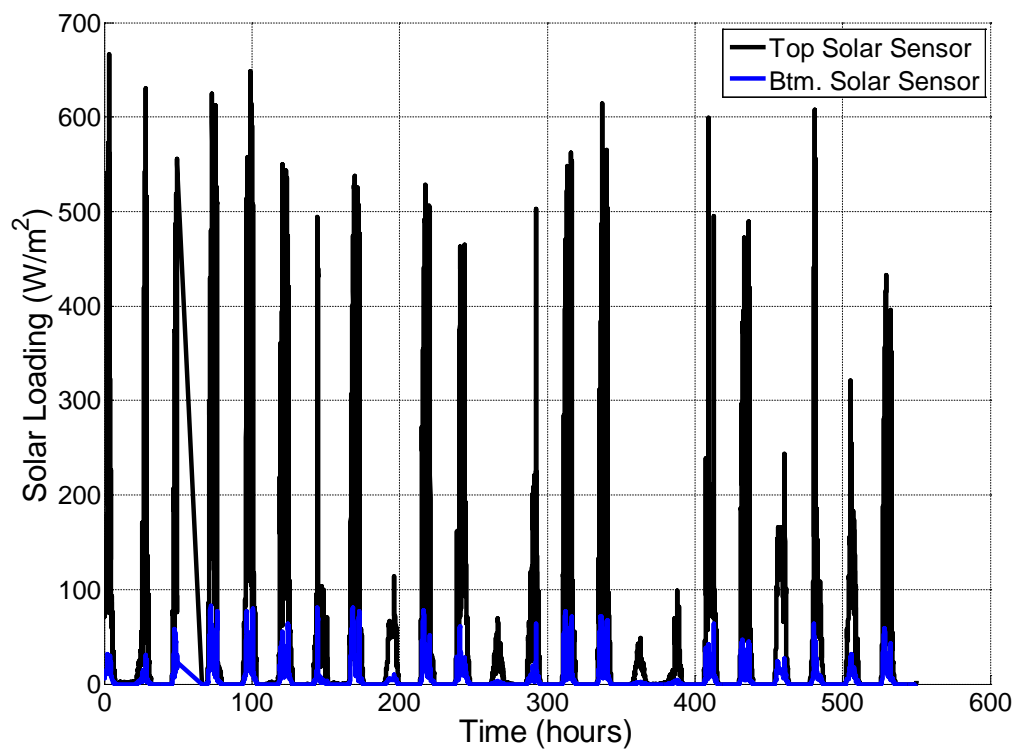

Figure C-16: NON-IFP Tank Exp. 5 Hold Time Simulation Tank Heat Transfer 


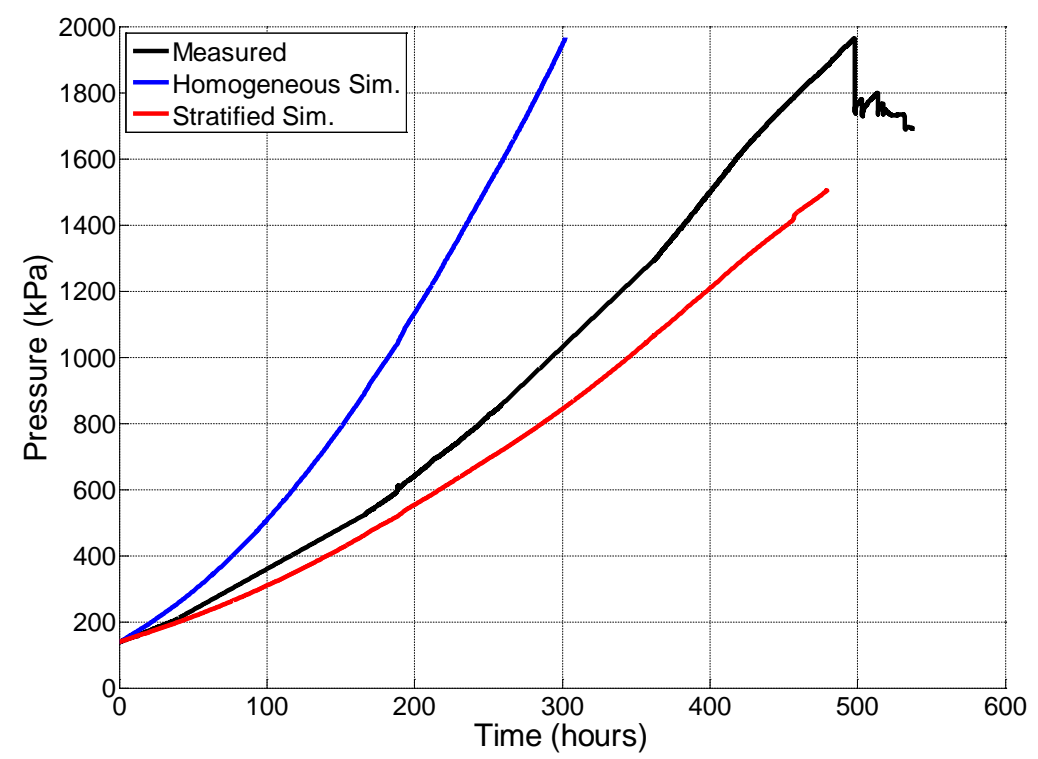

Figure C-17: NON-IFP Tank Exp. 6 Hold Time Simulation Pressure Comparison

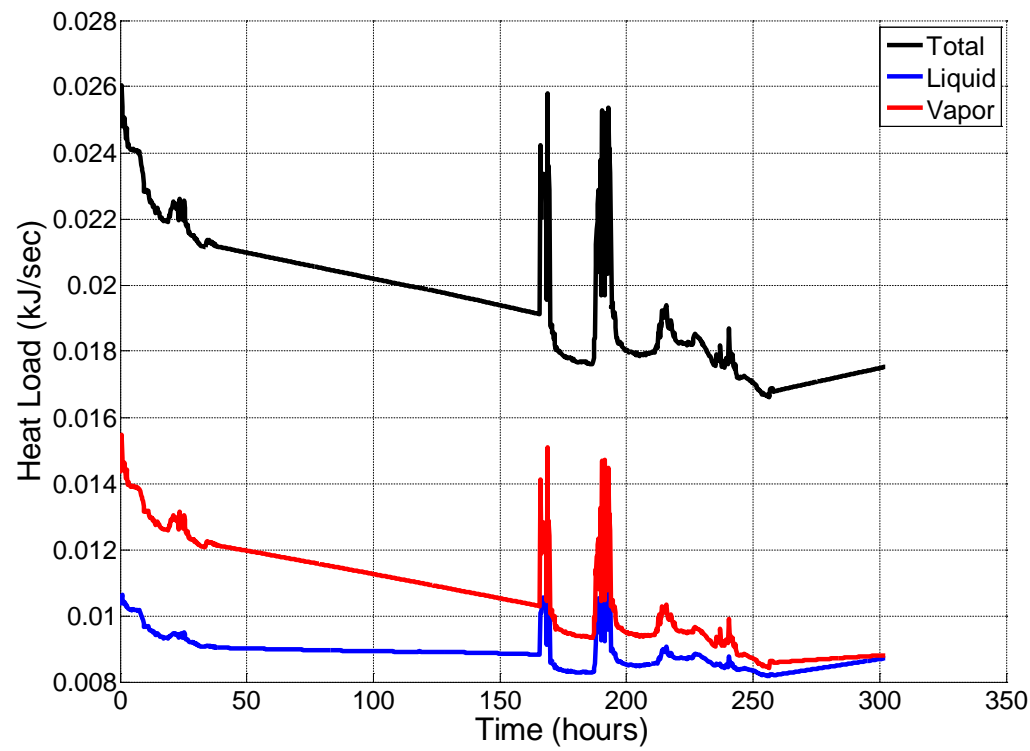

Figure C-18: NON-IFP Tank Exp. 6 Hold Time Simulation Tank Heat Transfer 


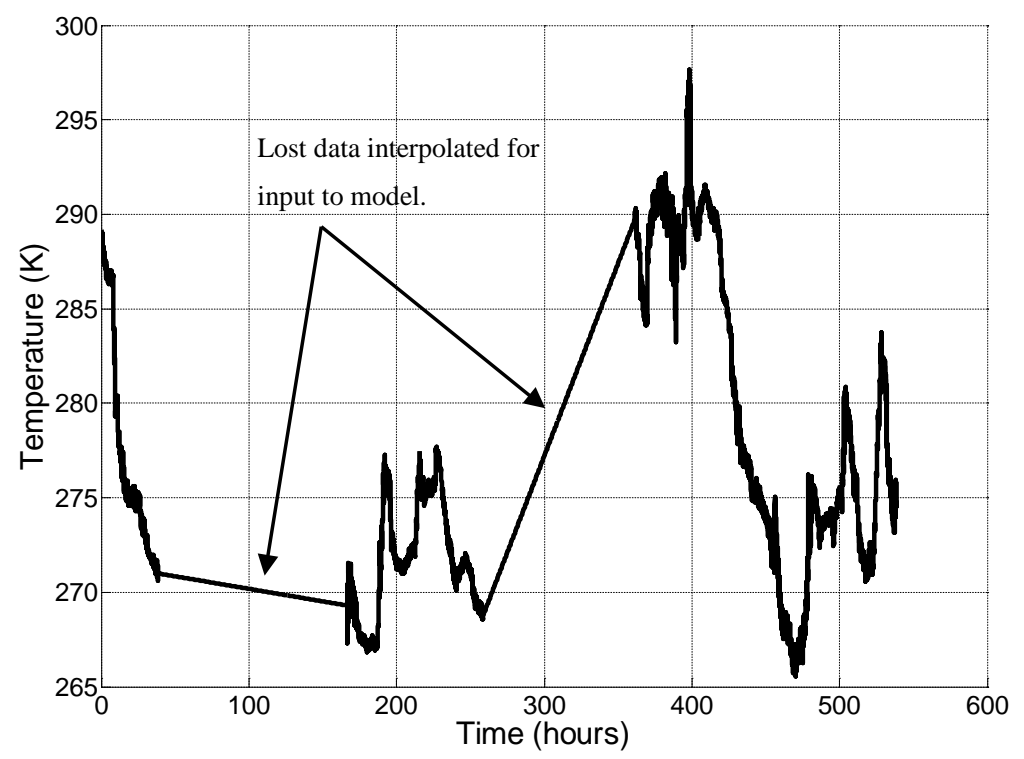

Figure C-19: NON-IFP Tank Exp. 6 Hold Time Simulation Ambient Temperature

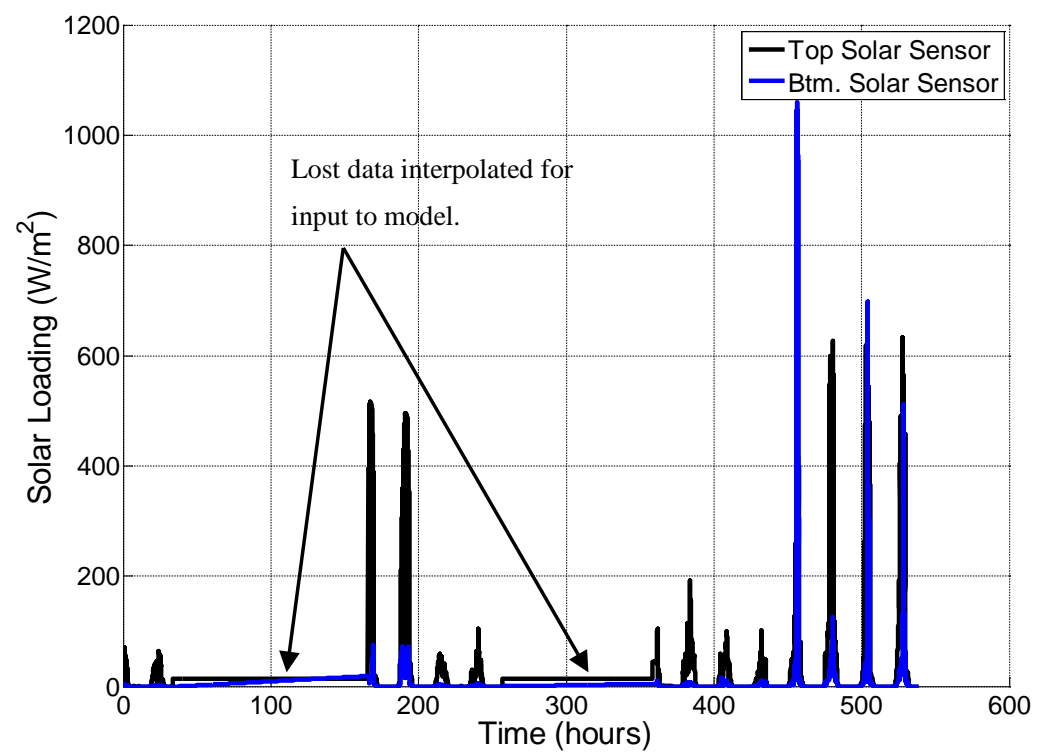

Figure C-20: NON-IFP Tank Exp. 6 Hold Time Simulation Tank Heat Transfer 


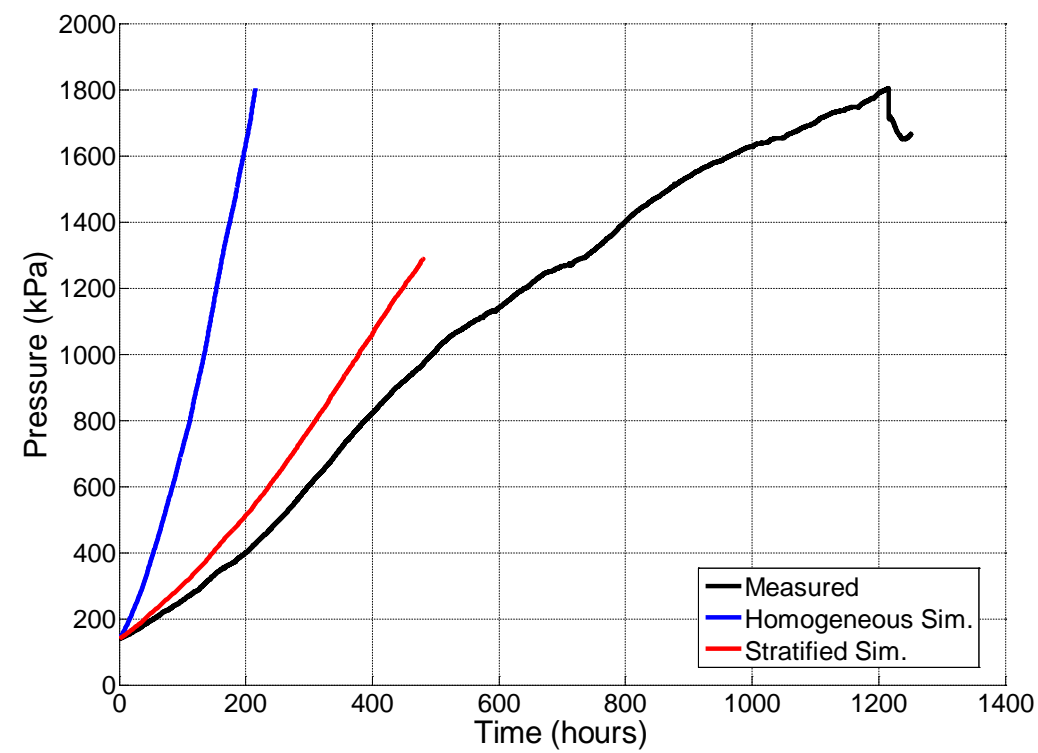

Figure C-21: NON-IFP Tank Exp. 7 Hold Time Simulation Pressure Comparison

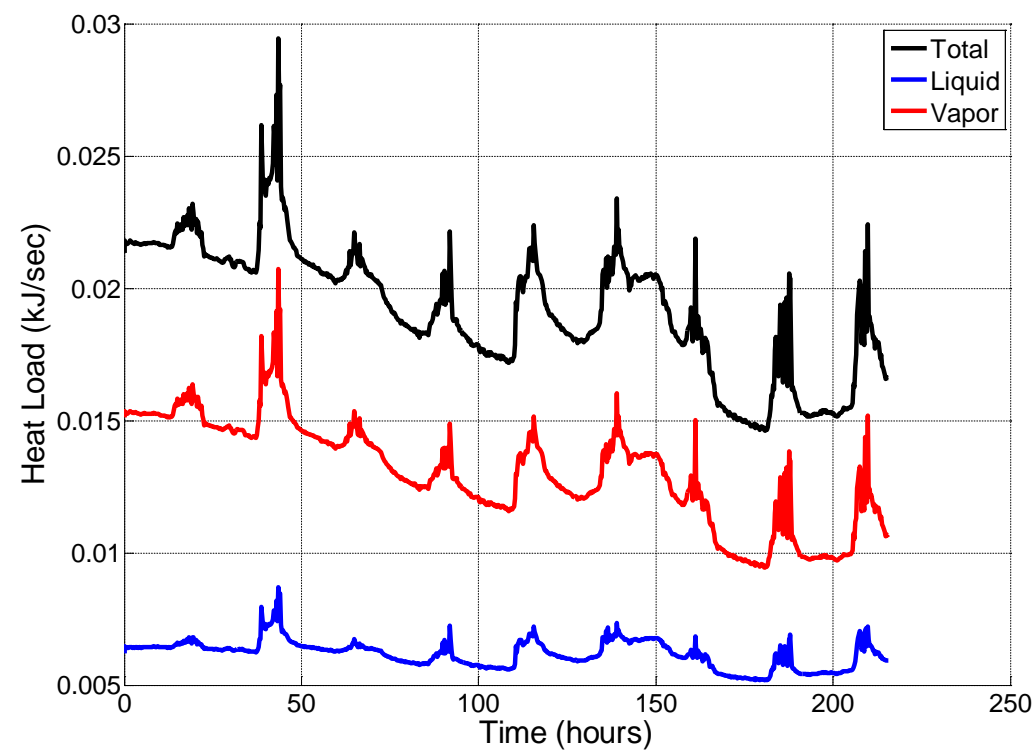

Figure C-22: NON-IFP Tank Exp. 7 Hold Time Simulation Tank Heat Transfer 


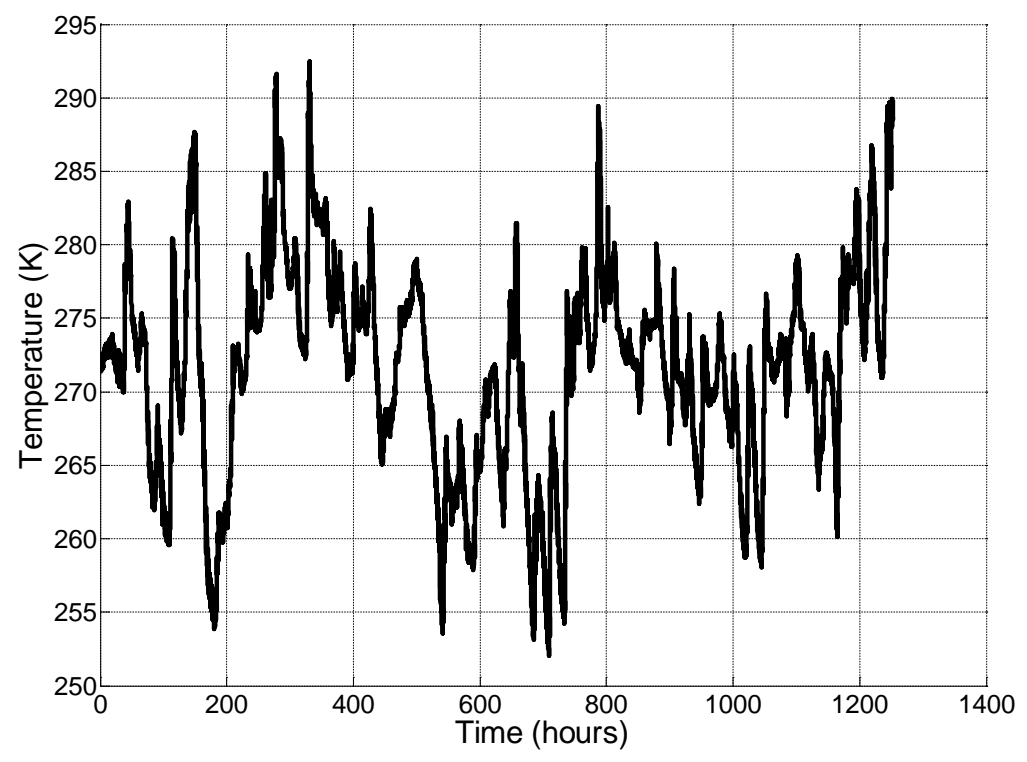

Figure C-23: NON-IFP Tank Exp. 7 Hold Time Simulation Ambient Temperature

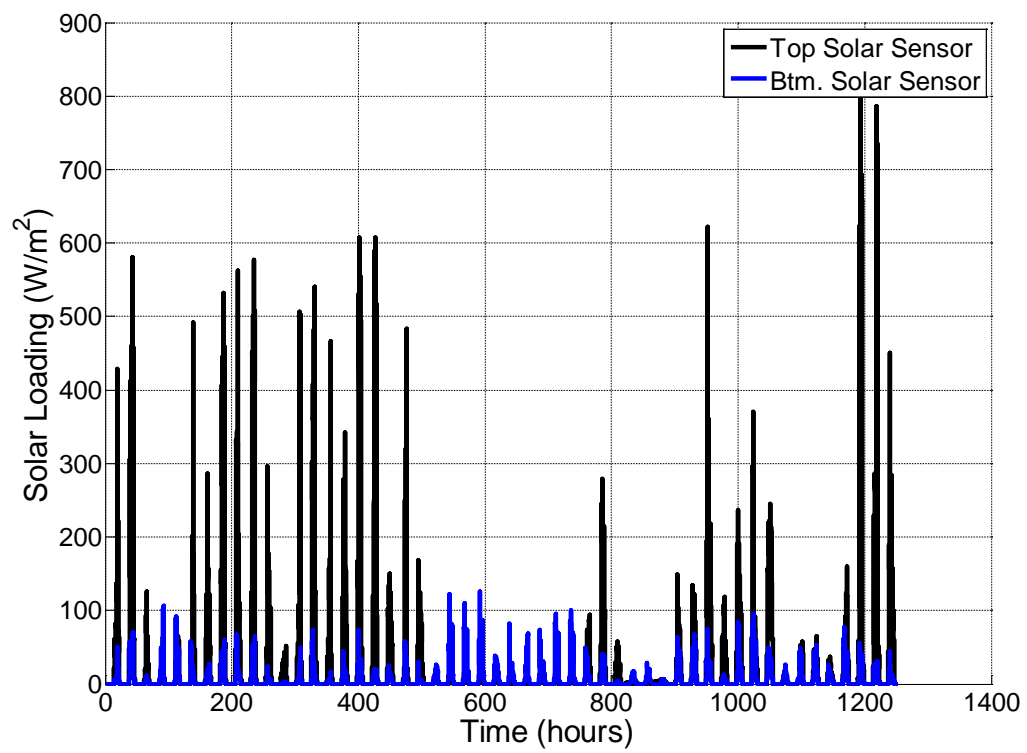

Figure C-24: NON-IFP Tank Exp. 7 Hold Time Simulation Tank Heat Transfer 


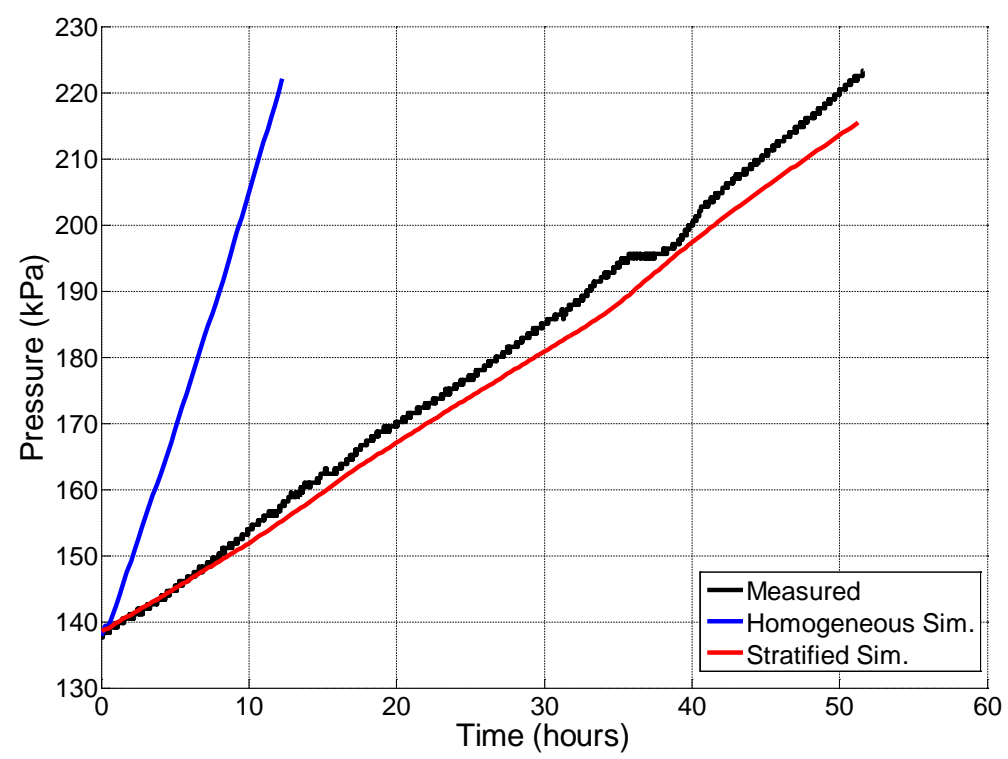

Figure C-25: NON-IFP Tank Exp. 8 Hold Time Simulation Pressure Comparison

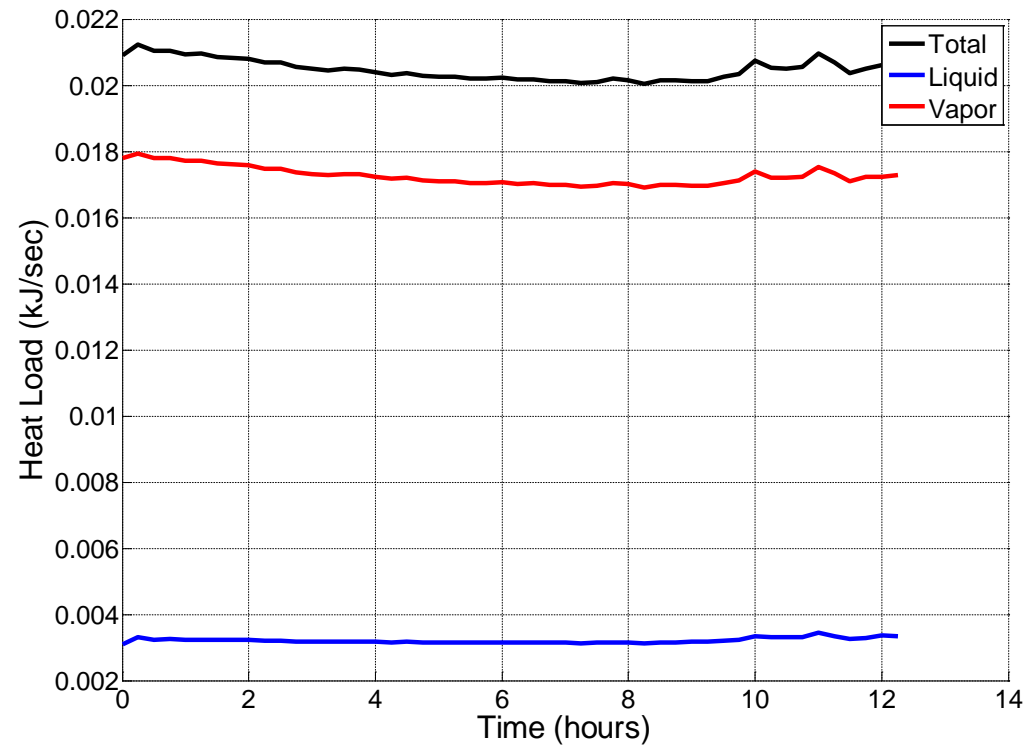

Figure C-26: NON-IFP Tank Exp. 8 Hold Time Simulation Tank Heat Transfer 


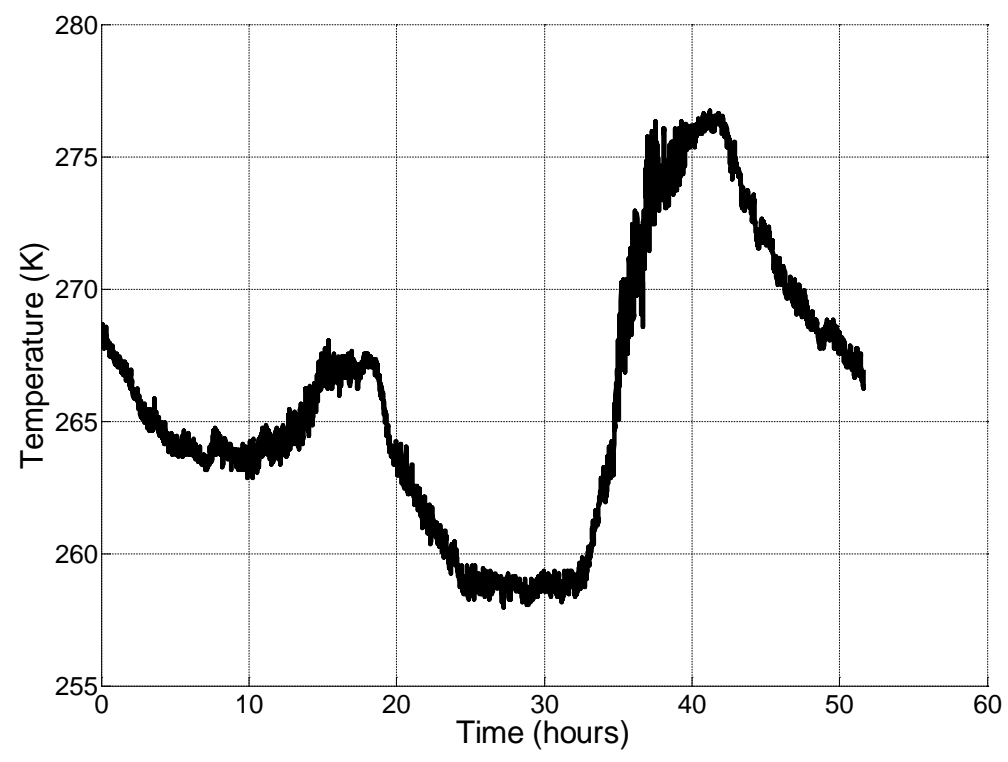

Figure C-27: NON-IFP Tank Exp. 8 Hold Time Simulation Ambient Temperature

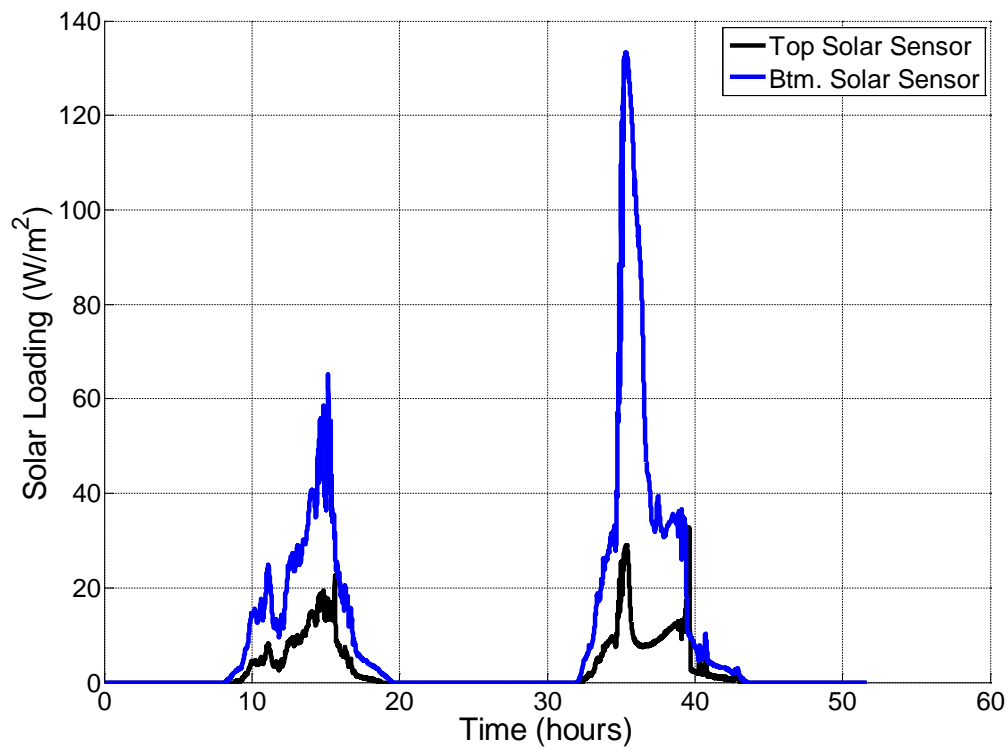

Figure C-28: NON-IFP Tank Exp. 8 Hold Time Simulation Tank Heat Transfer 


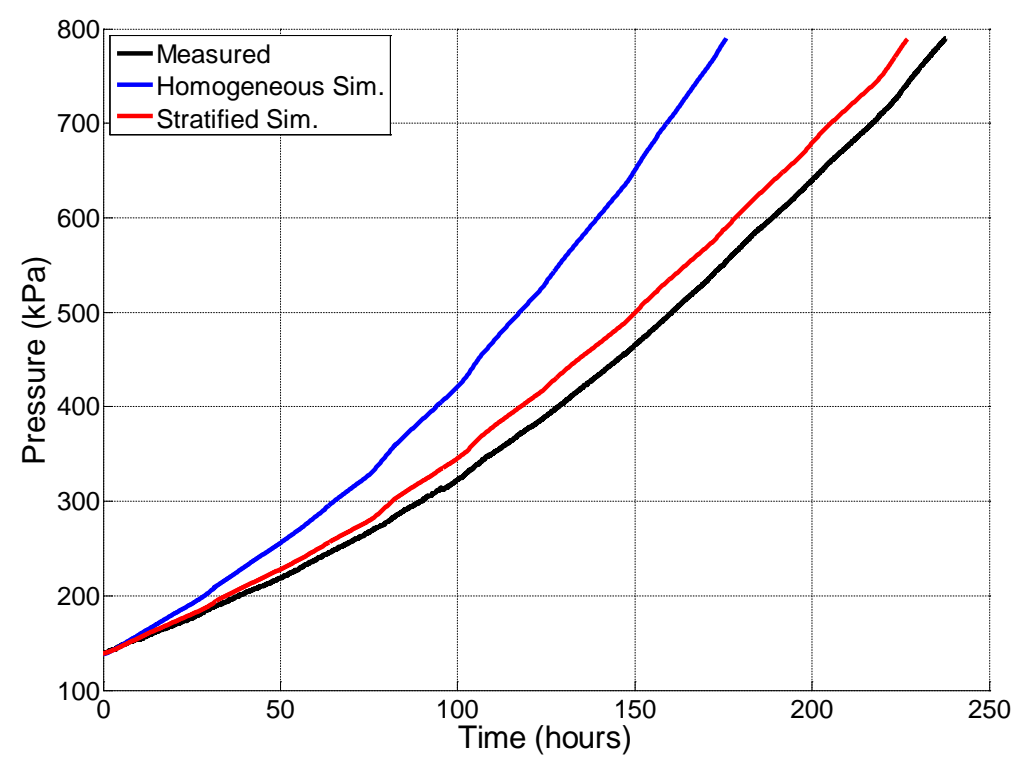

Figure C-29: NON-IFP Tank Exp. 9 Hold Time Simulation Pressure Comparison

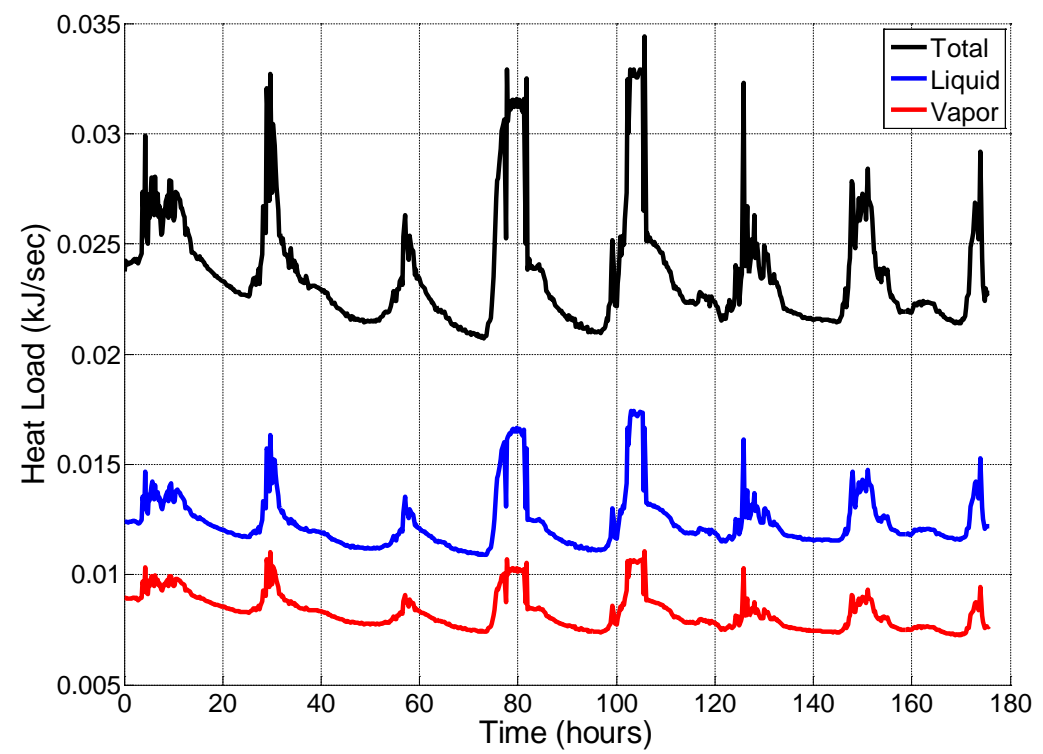

Figure C-30: NON-IFP Tank Exp. 9 Hold Time Simulation Tank Heat Transfer 


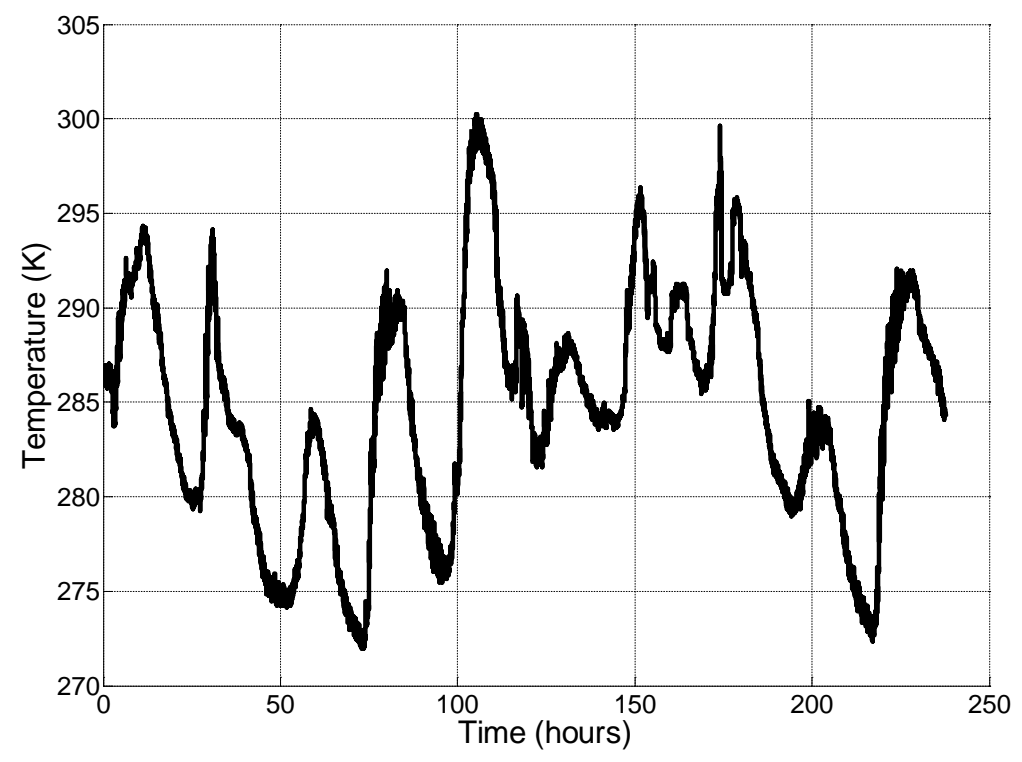

Figure C-31: NON-IFP Tank Exp. 9 Hold Time Simulation Ambient Temperature

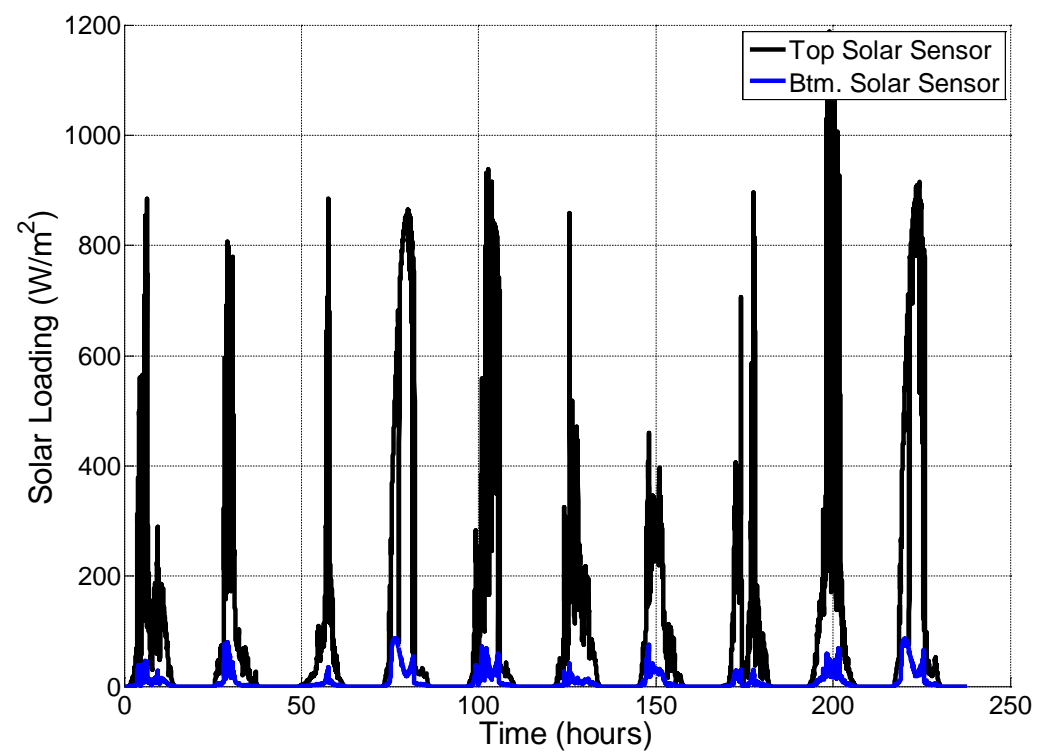

Figure C-32: NON-IFP Tank Exp. 9 Hold Time Simulation Tank Heat Transfer 


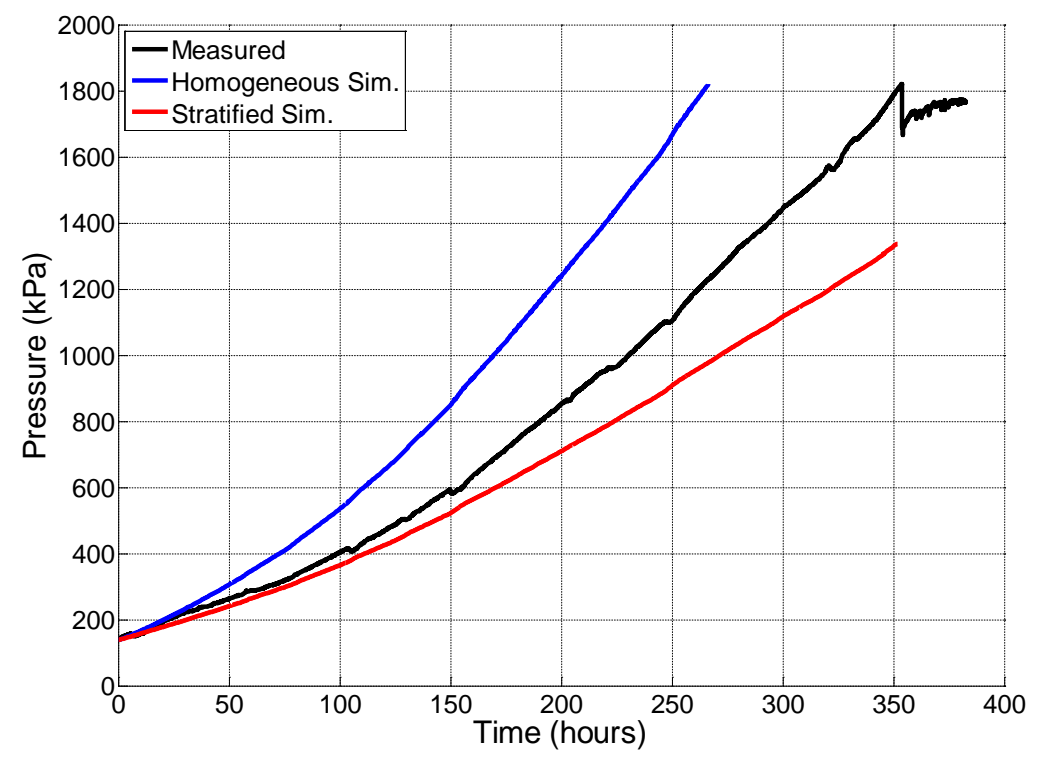

Figure C-33: IFP Tank Exp. 2 Hold Time Simulation Pressure Comparison

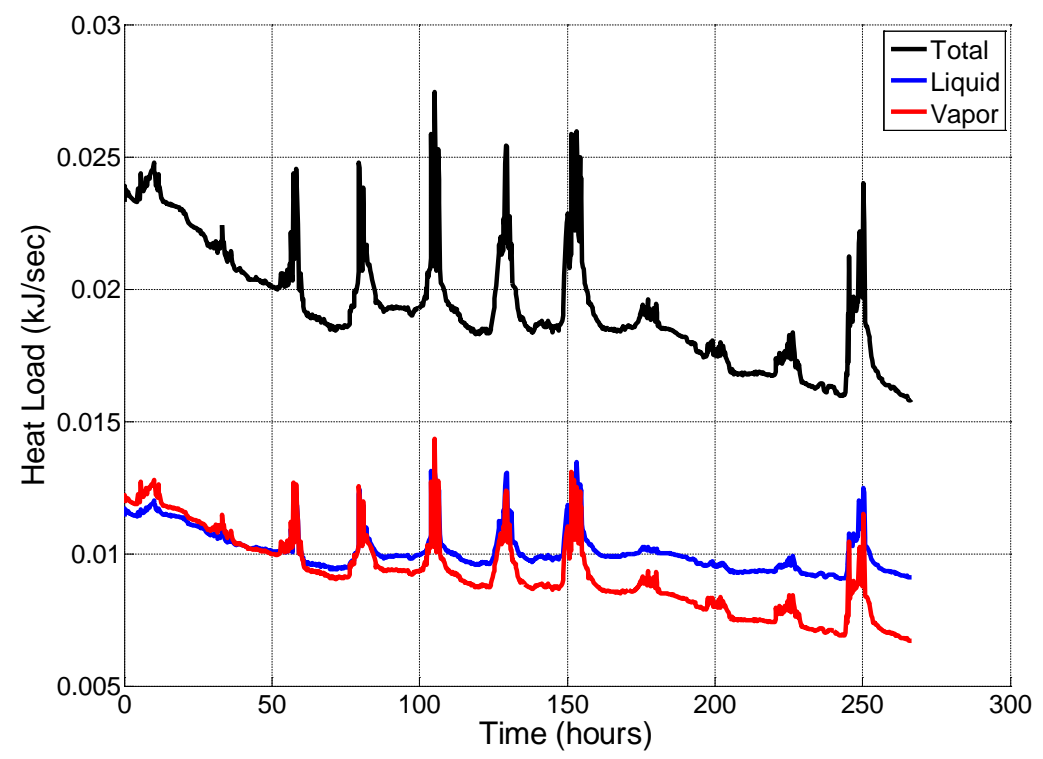

Figure C-34: IFP Tank Exp. 2 Hold Time Simulation Tank Heat Transfer 


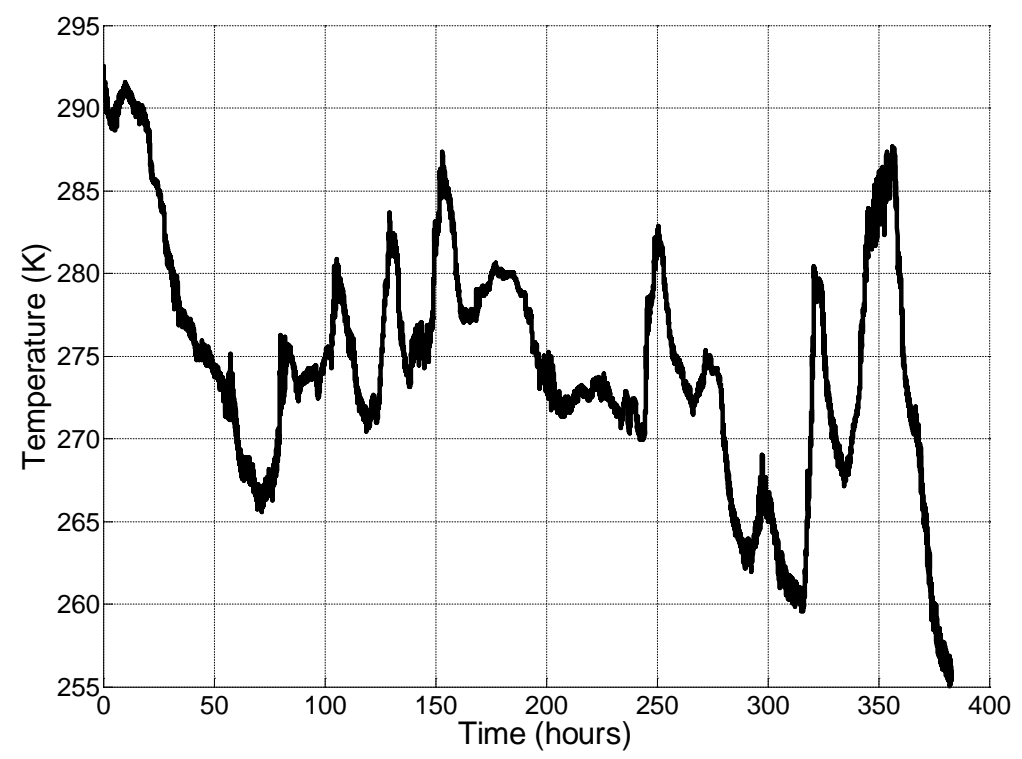

Figure C-35: IFP Tank Exp. 2 Hold Time Simulation Ambient Temperature

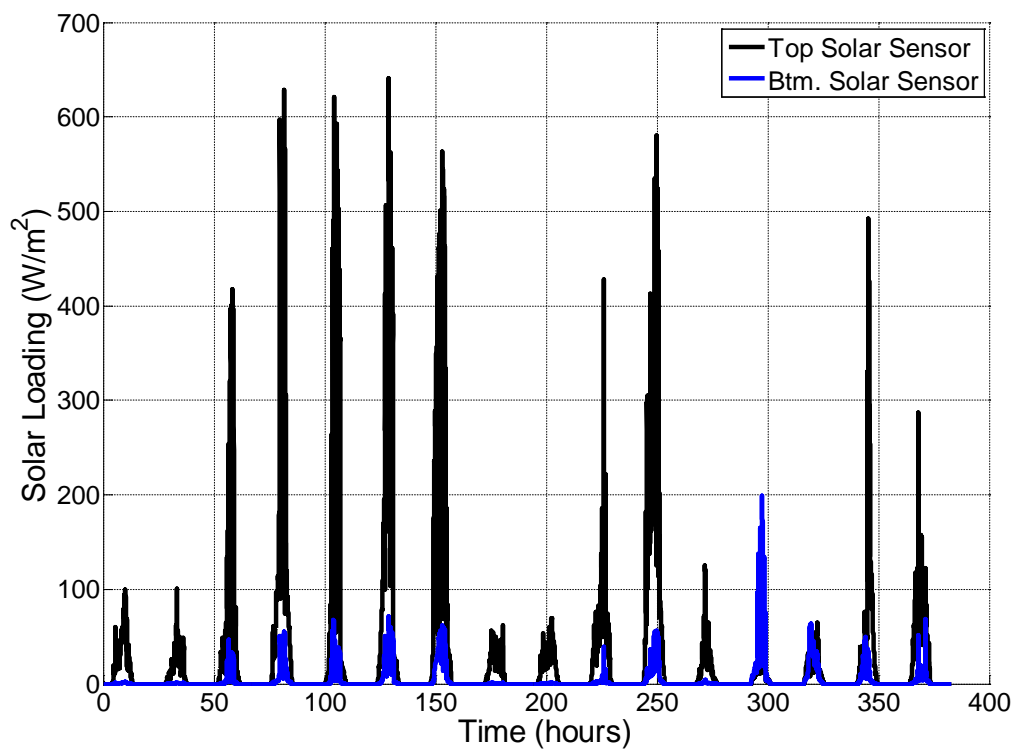

Figure C-36: IFP Tank Exp. 2 Hold Time Simulation Tank Heat Transfer 


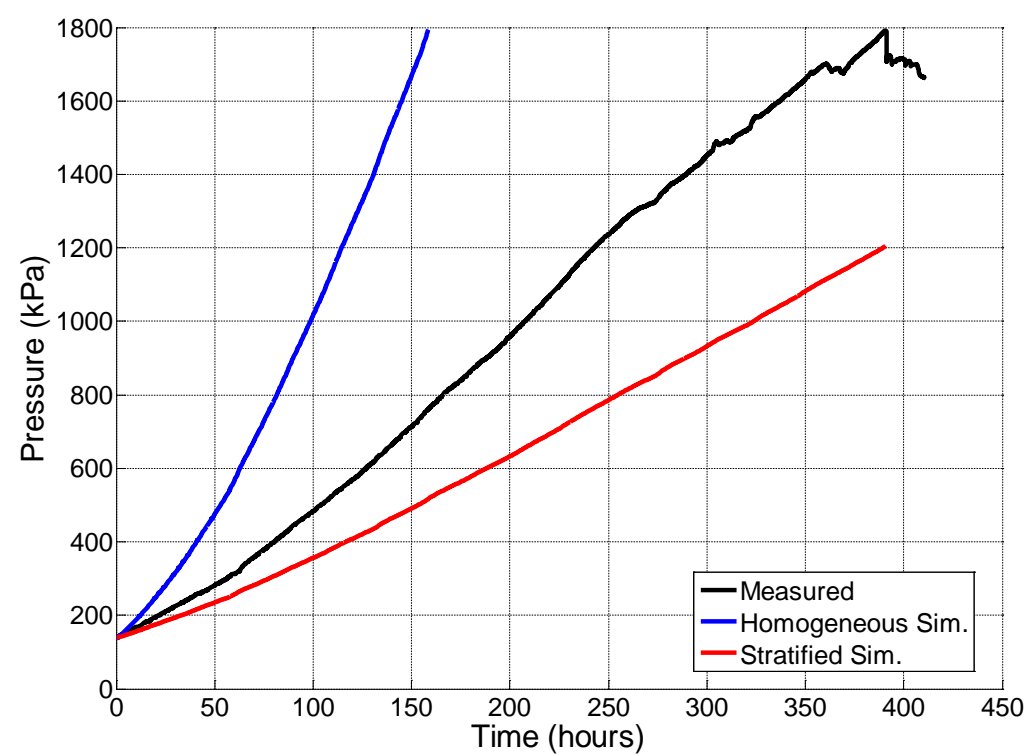

Figure C-37: IFP Tank Exp. 3 Hold Time Simulation Pressure Comparison

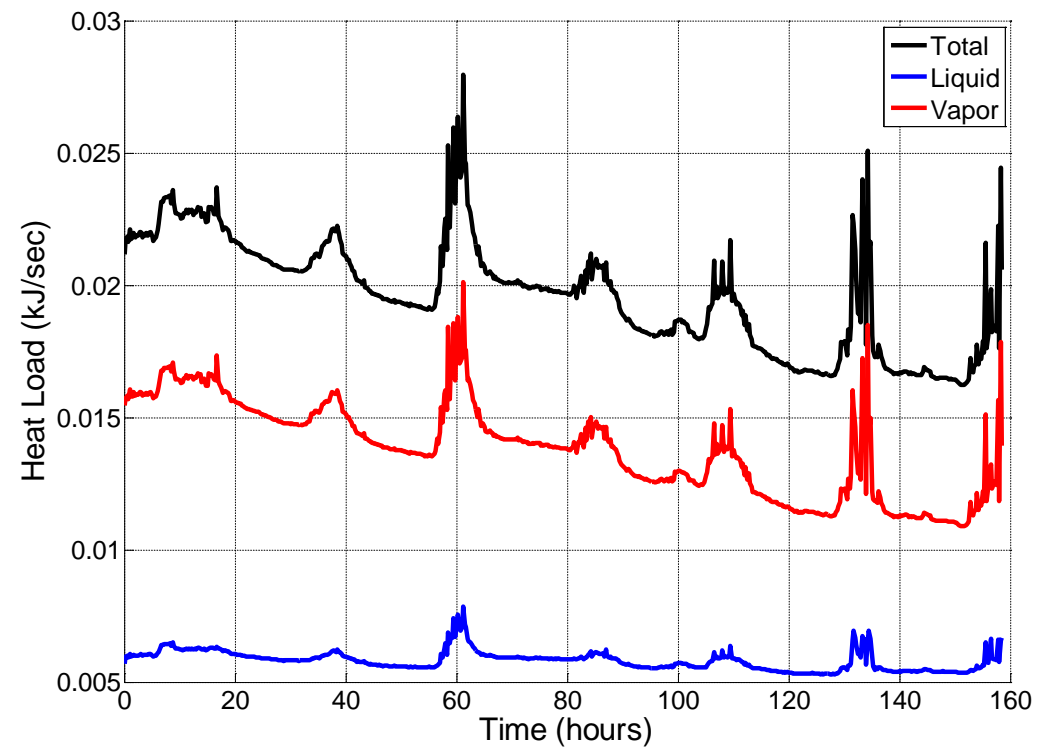

Figure C-38: IFP Tank Exp. 3 Hold Time Simulation Tank Heat Transfer 


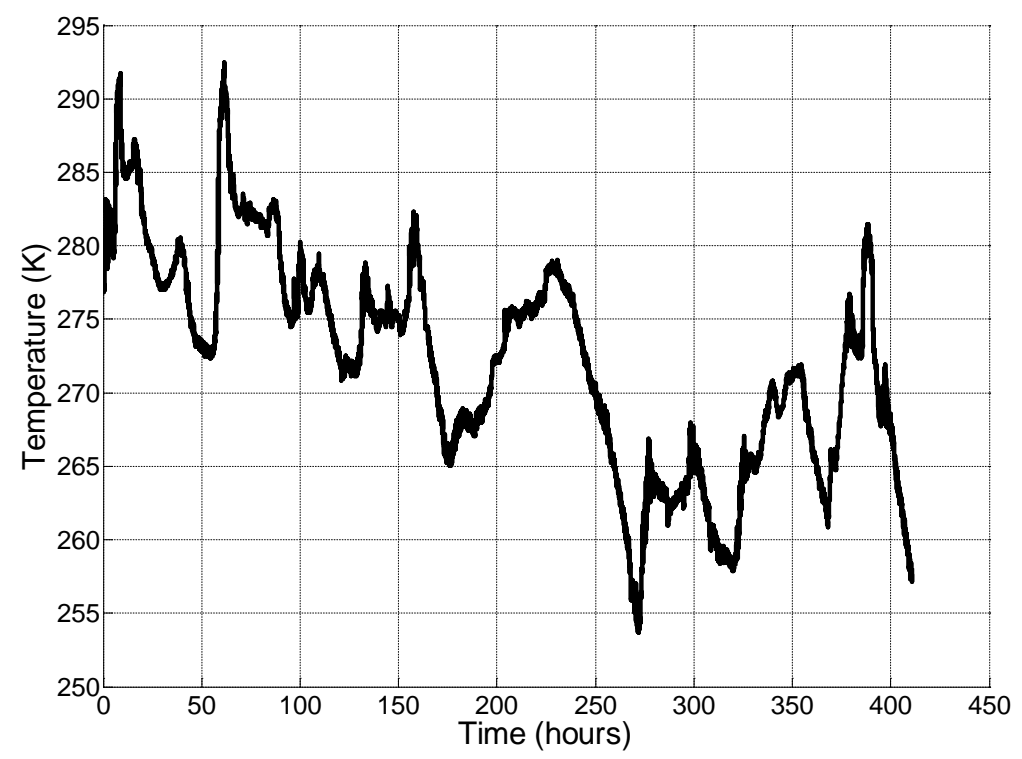

Figure C-39: IFP Tank Exp. 3 Hold Time Simulation Ambient Temperature

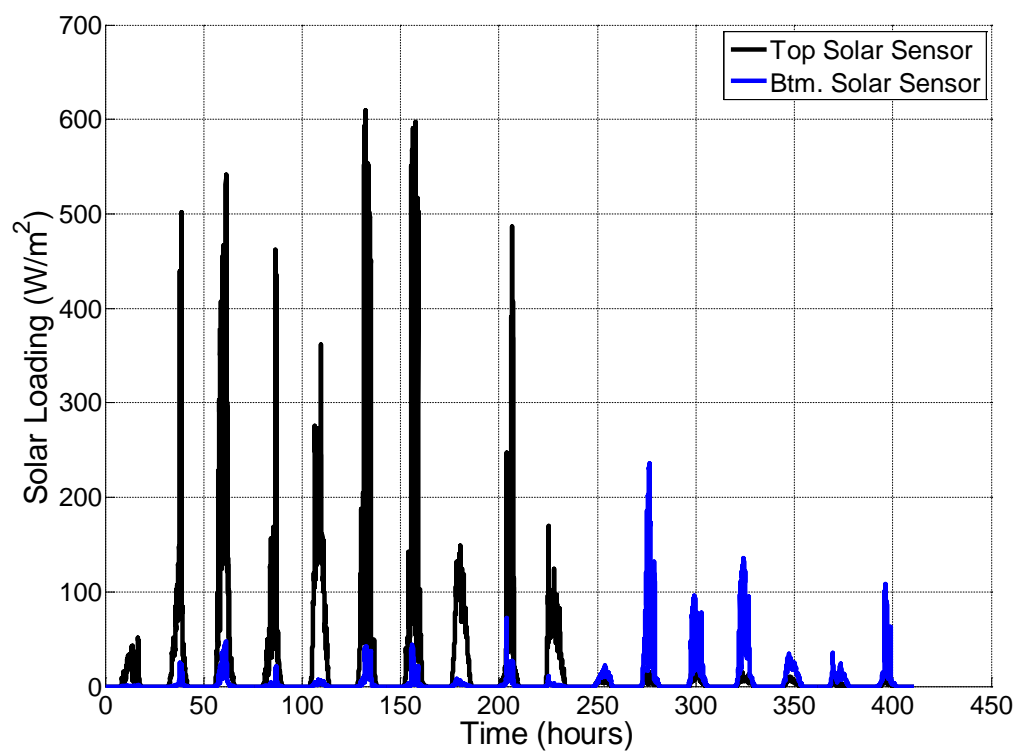

Figure C-40: IFP Tank Exp. 3 Hold Time Simulation Tank Heat Transfer 


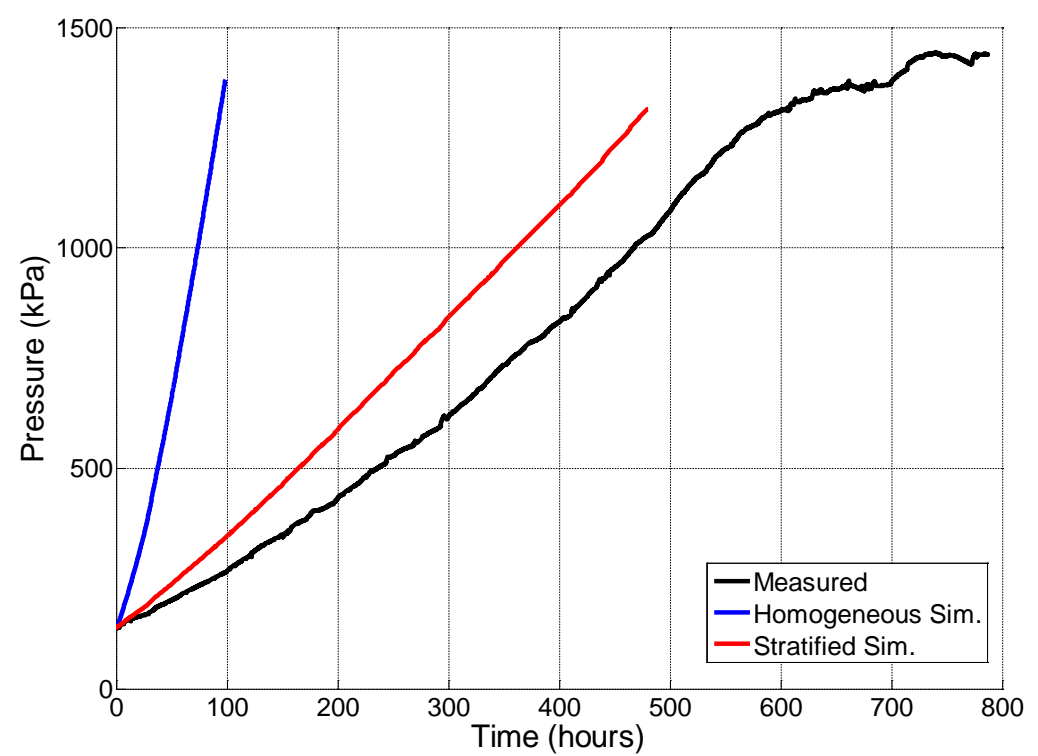

Figure C-41: IFP Tank Exp. 4 Hold Time Simulation Pressure Comparison

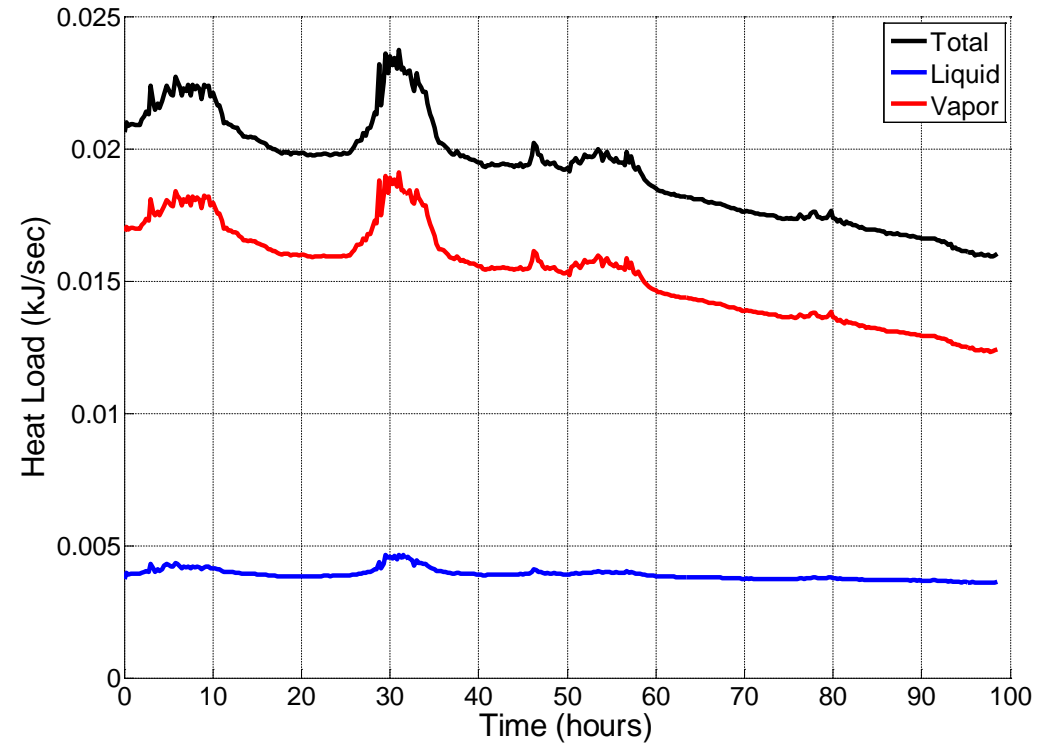

Figure C-42: IFP Tank Exp. 4 Hold Time Simulation Tank Heat Transfer 


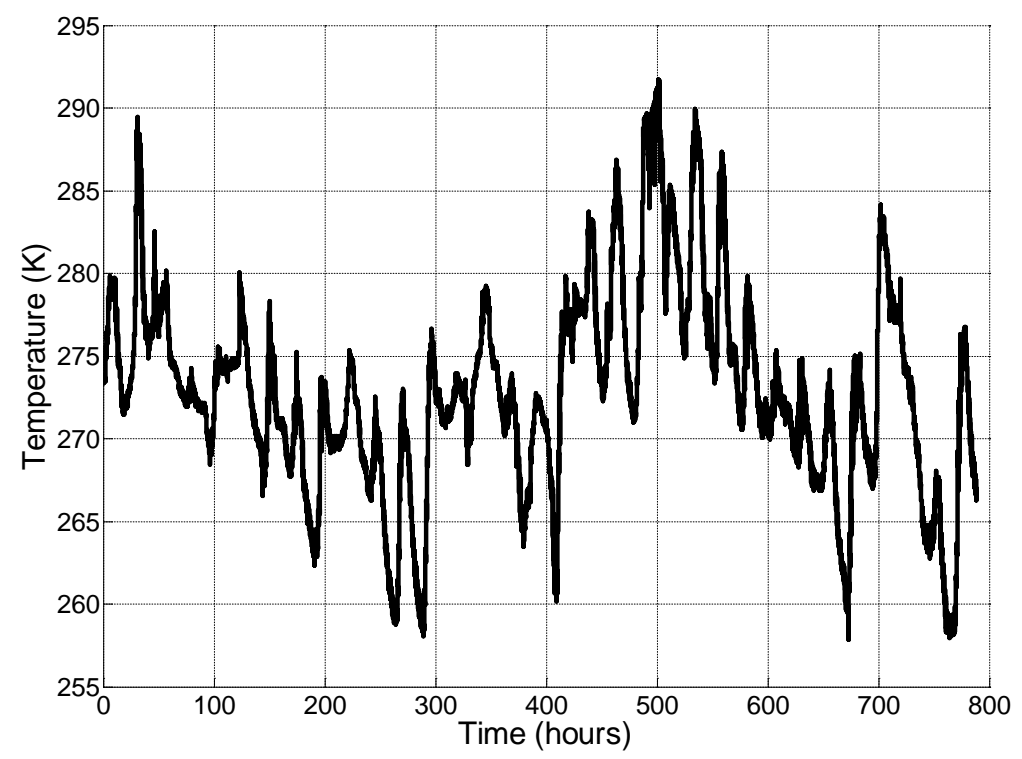

Figure C-43: IFP Tank Exp. 4 Hold Time Simulation Ambient Temperature

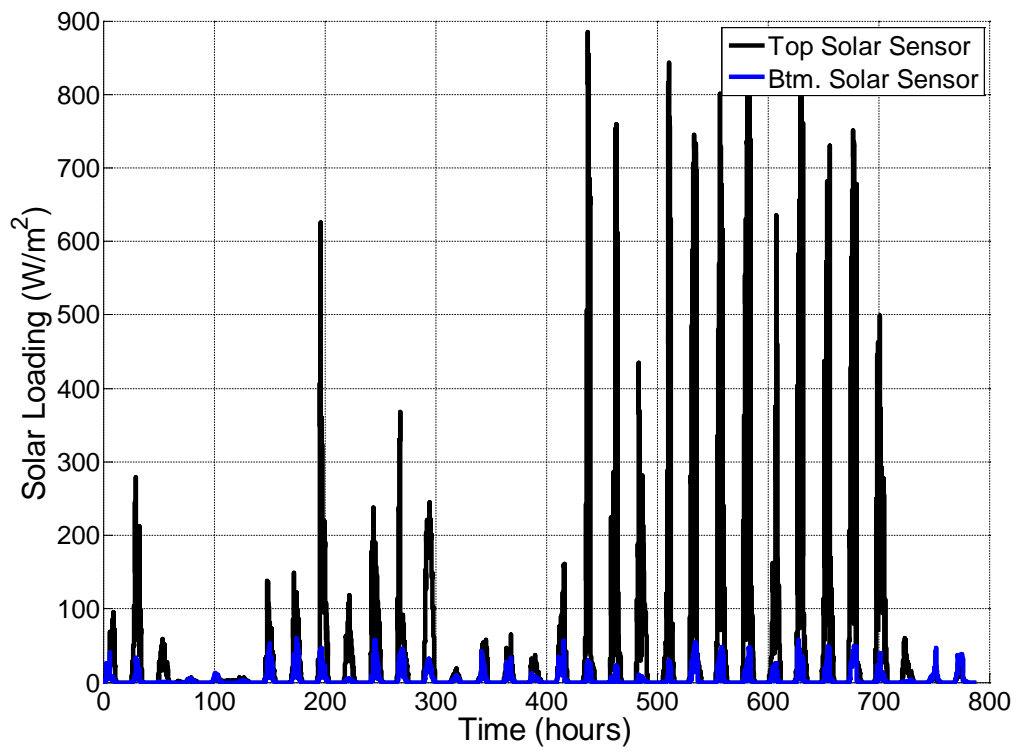

Figure C-44: IFP Tank Exp. 4 Hold Time Simulation Tank Heat Transfer 


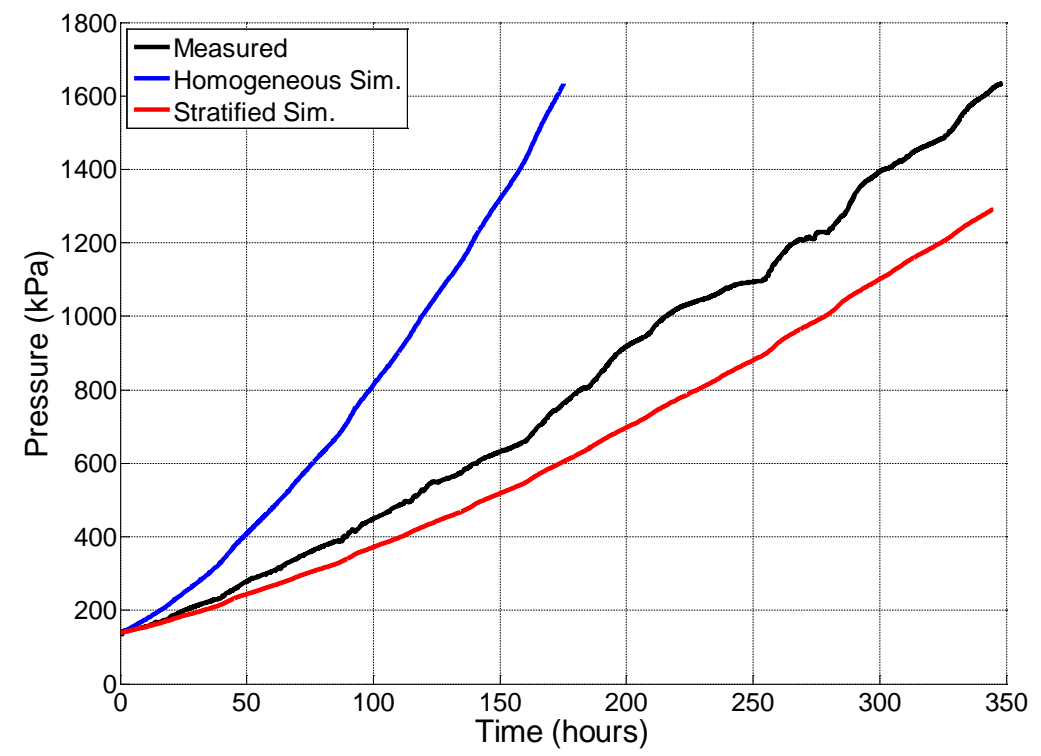

Figure C-45: IFP Tank Exp. 5 Hold Time Simulation Pressure Comparison

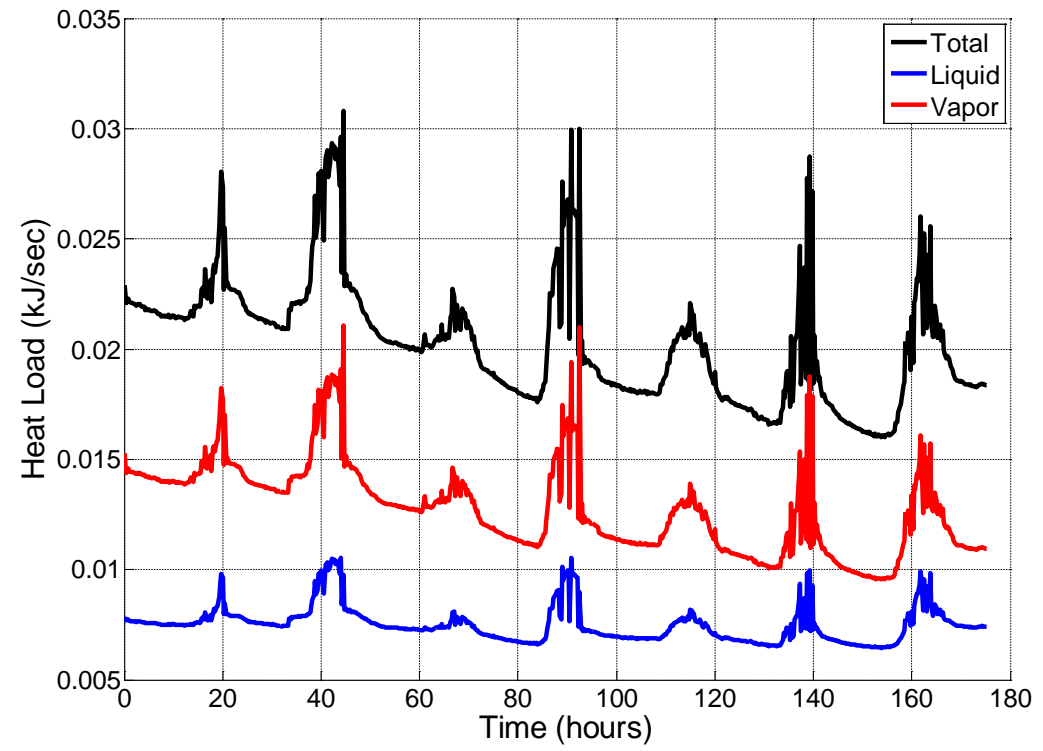

Figure C-46: IFP Tank Exp. 5 Hold Time Simulation Tank Heat Transfer 


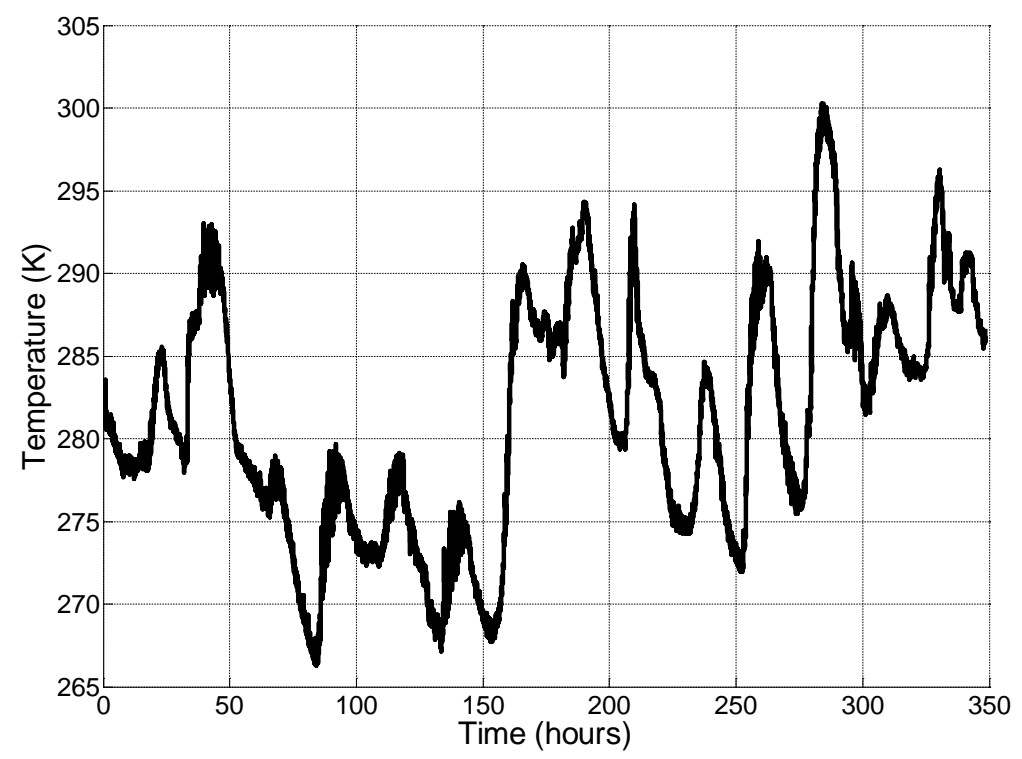

Figure C-47: IFP Tank Exp. 5 Hold Time Simulation Ambient Temperature

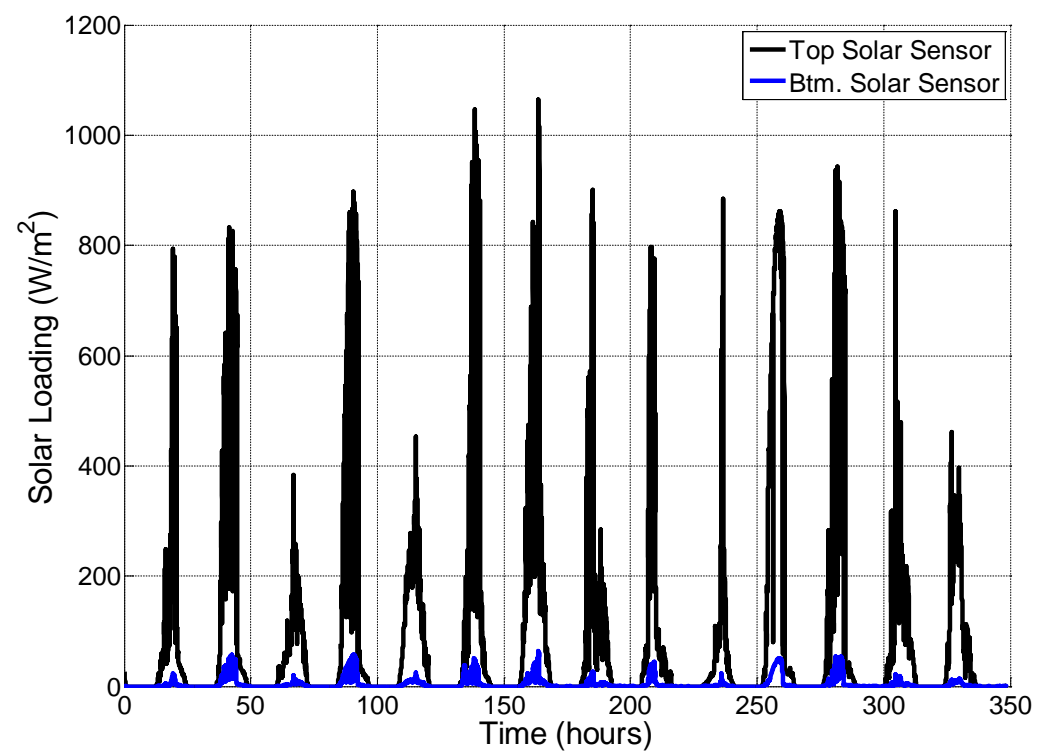

Figure C-48: IFP Tank Exp. 5 Hold Time Simulation Tank Heat Transfer 


\section{C-2 Audit Simulations}

\section{$\underline{\text { Audit } 1 \text { Segment } 1}$}

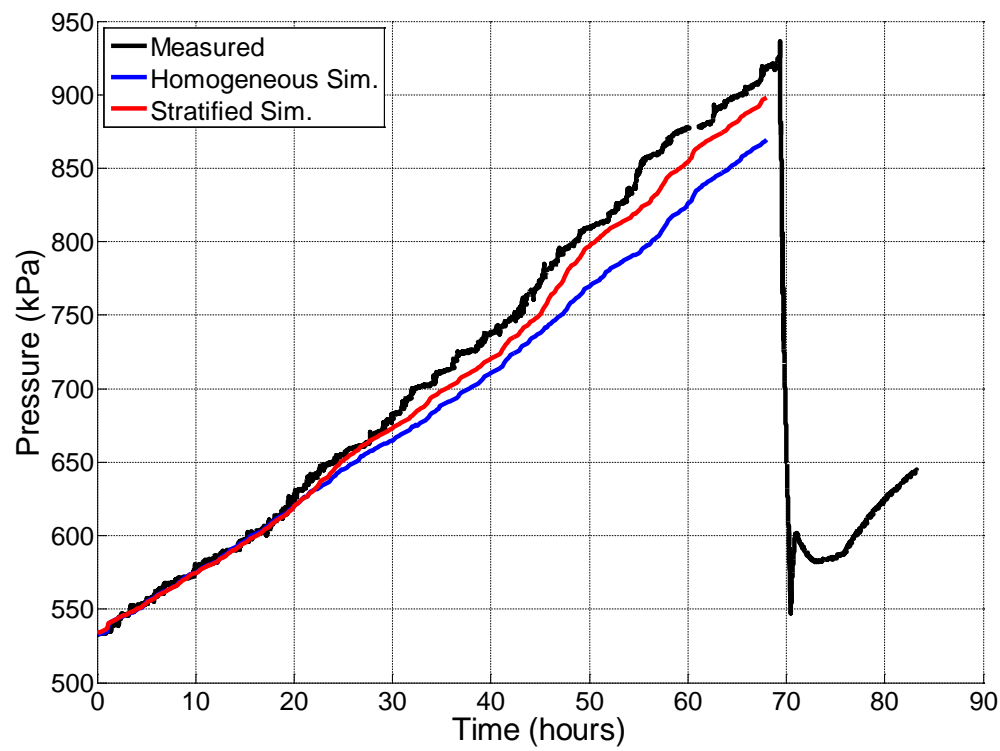

Figure C-49: Audit 1 Segment 1 Simulation Pressure Comparison

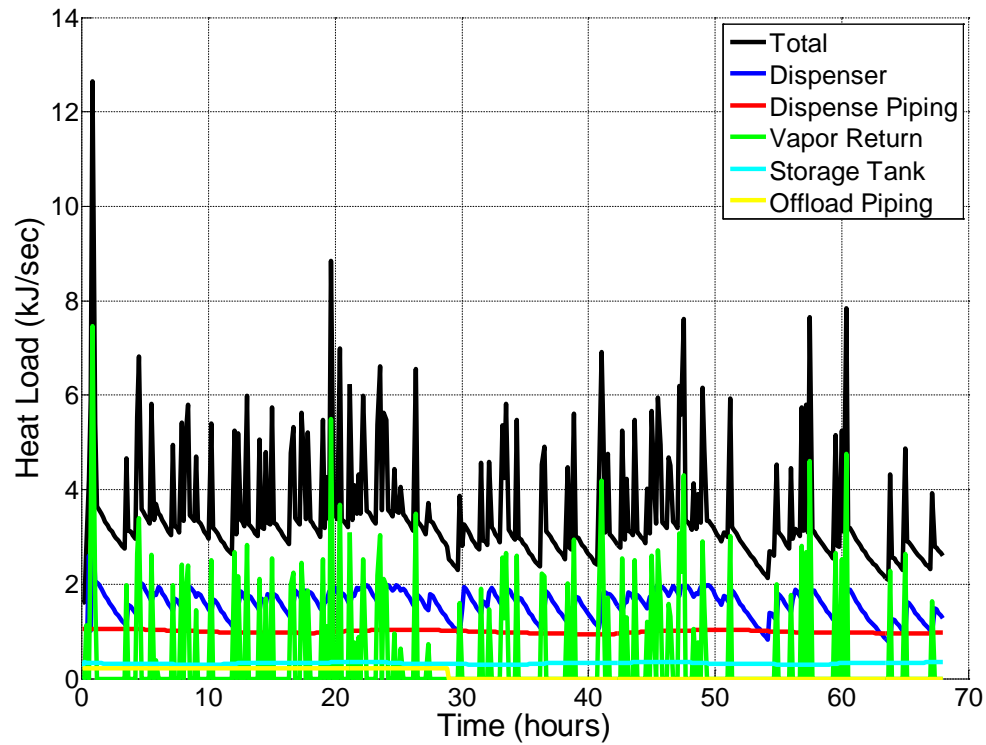

Figure C-50: Audit 1 Segment 1 Simulation Heat Transfer 


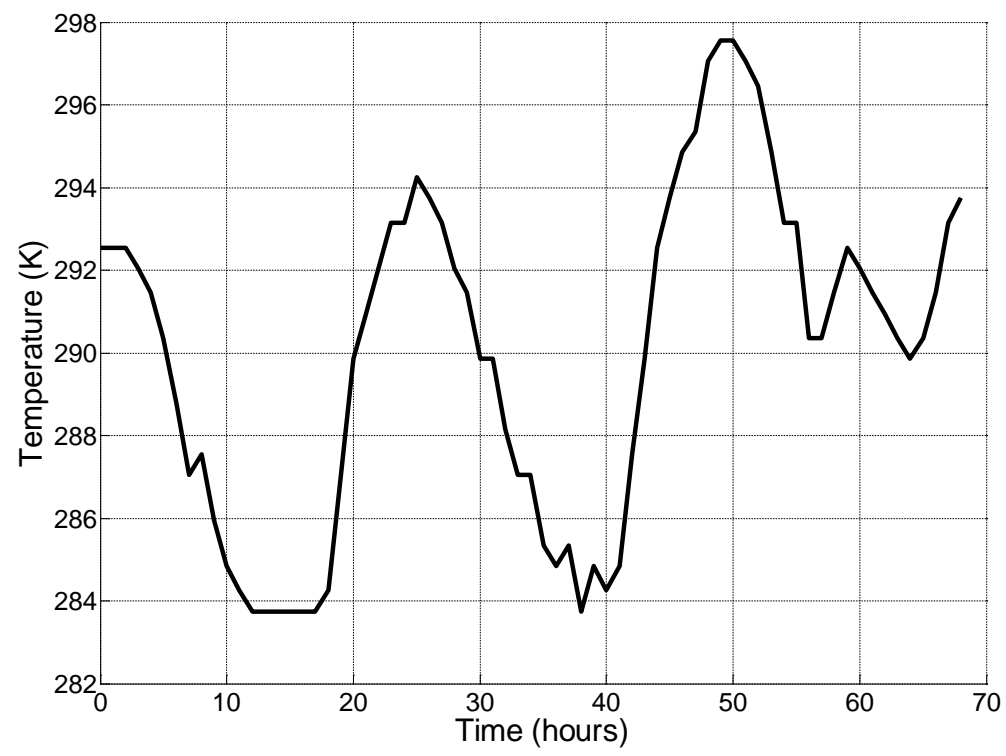

Figure C-51: Audit 1 Segment 1 Ambient Temperature
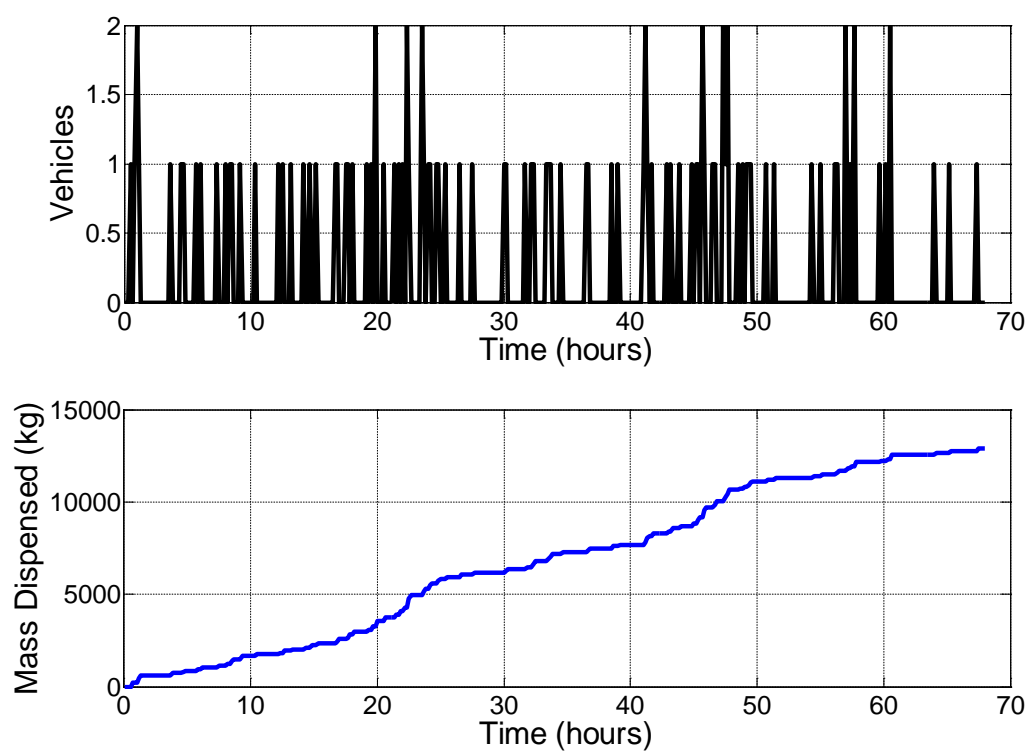

Figure C-52: Audit 1 Segment 1 Simulation Fleet Arrival Times and Mass Dispensed 


\section{Audit 1 Segment 2}

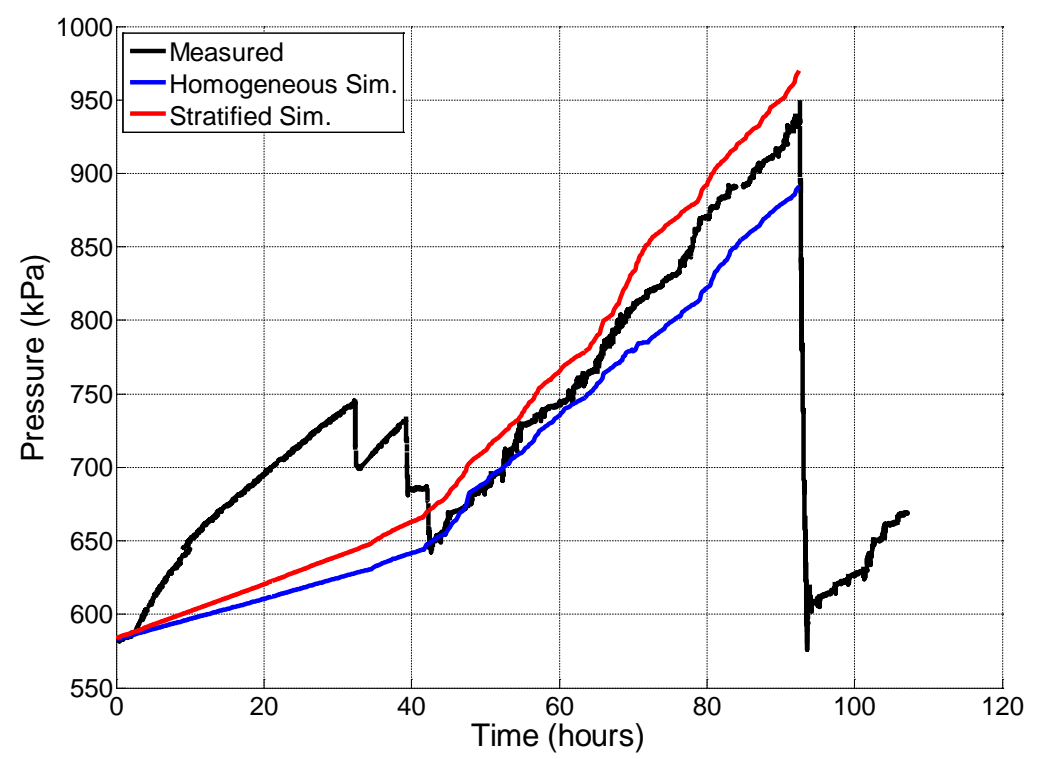

Figure C-53: Audit 1 Segment 2 Simulation Pressure Comparison

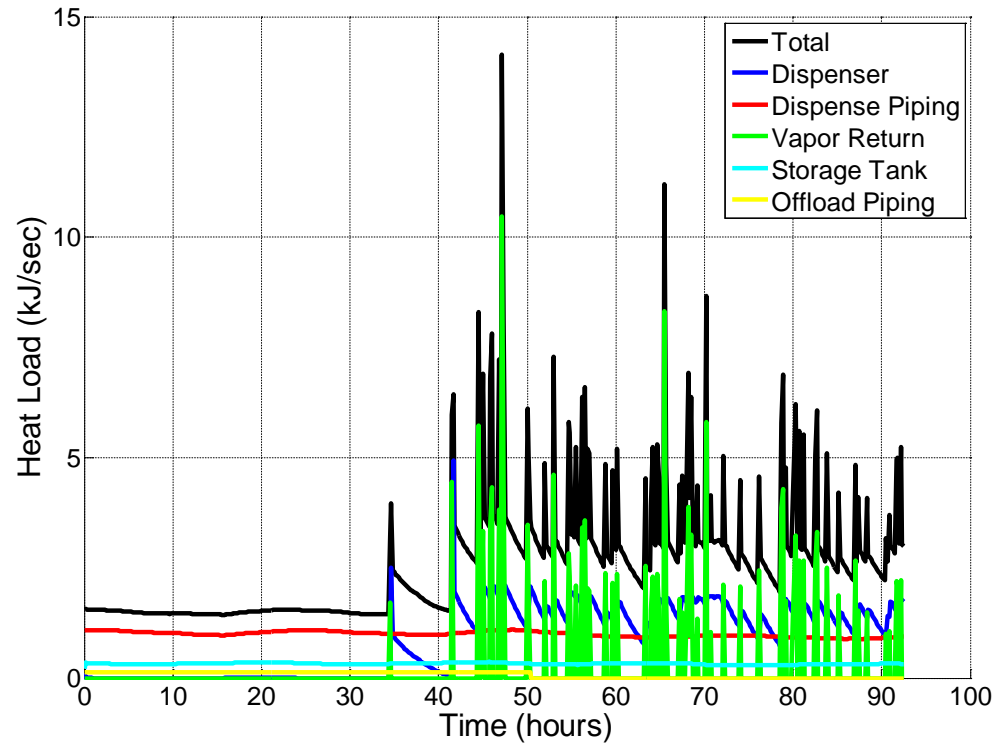

Figure C-54: Audit 1 Segment 2 Simulation Heat Transfer 

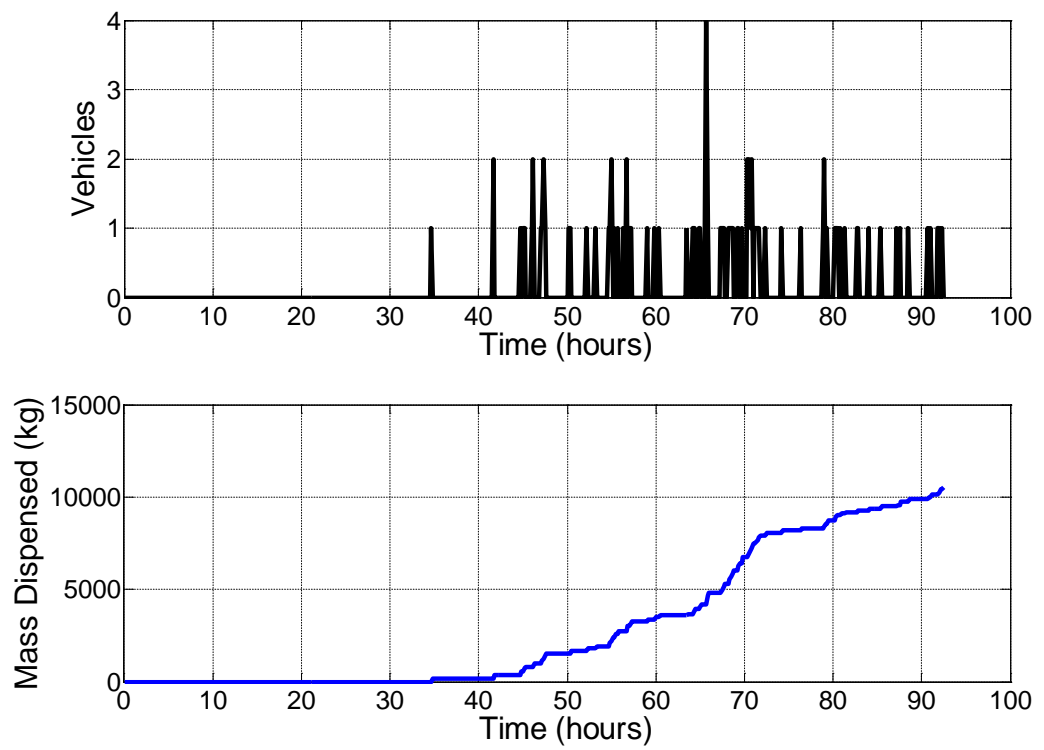

Figure C-55: Audit 1 Segment 2 Simulation Fleet Arrival Times and Mass Dispensed

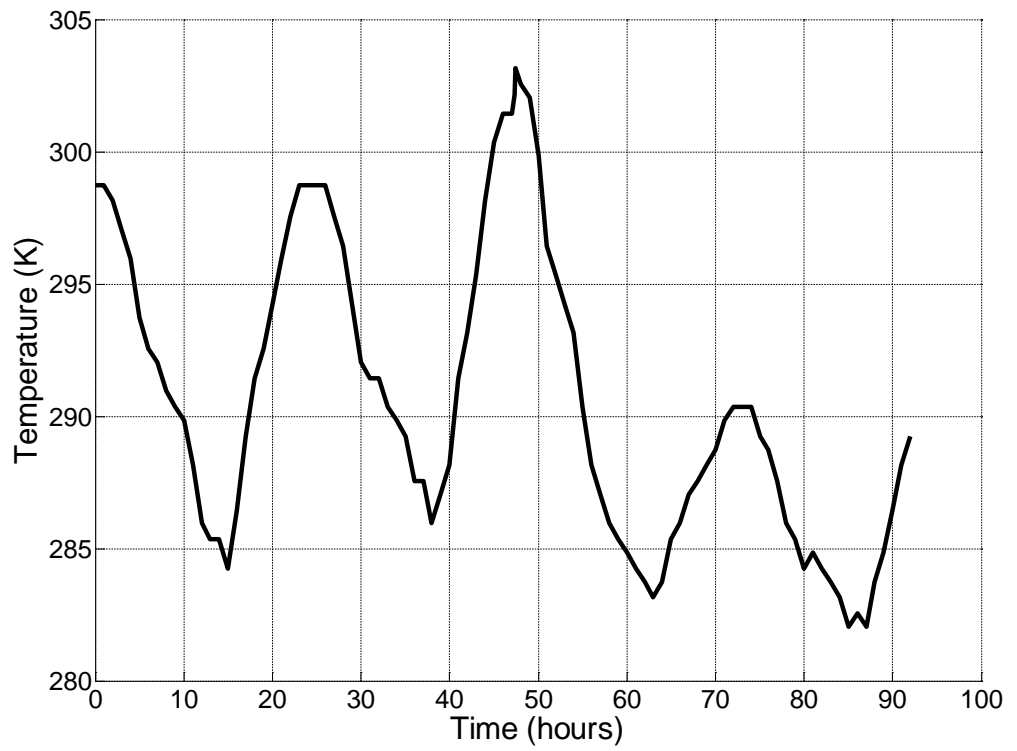

Figure C-56: Audit 1 Segment 2 Ambient Temperature 


\section{$\underline{\text { Audit } 1 \text { Segment } 3}$}

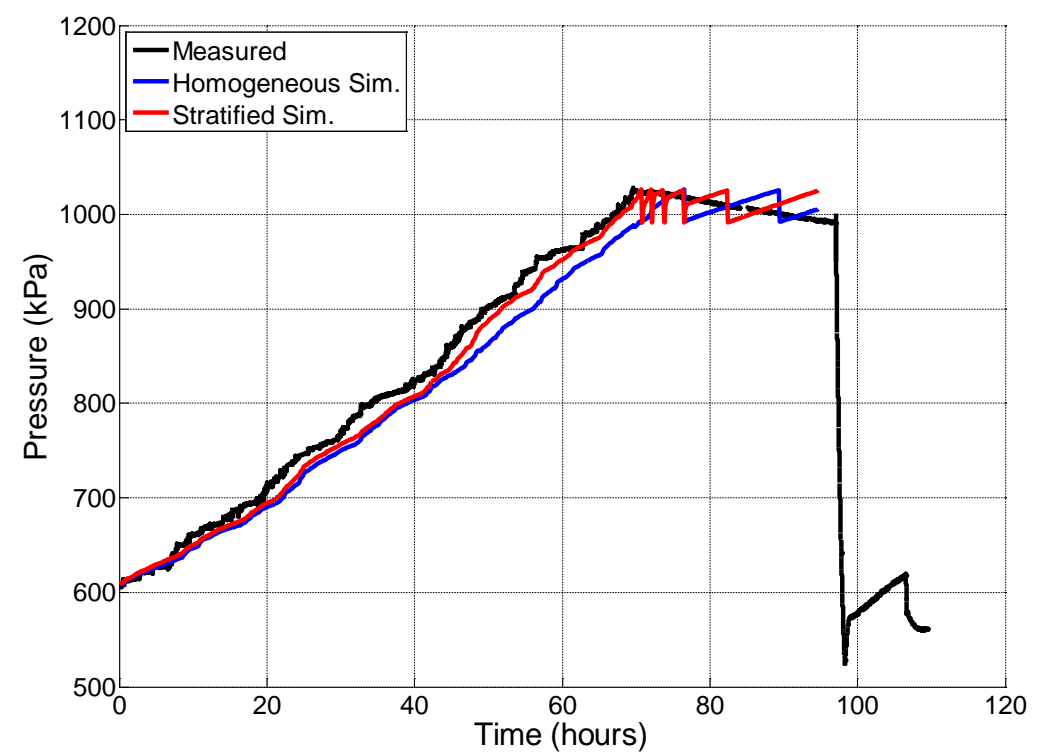

Figure C-57: Audit 1 Segment 3 Simulation Pressure Comparison

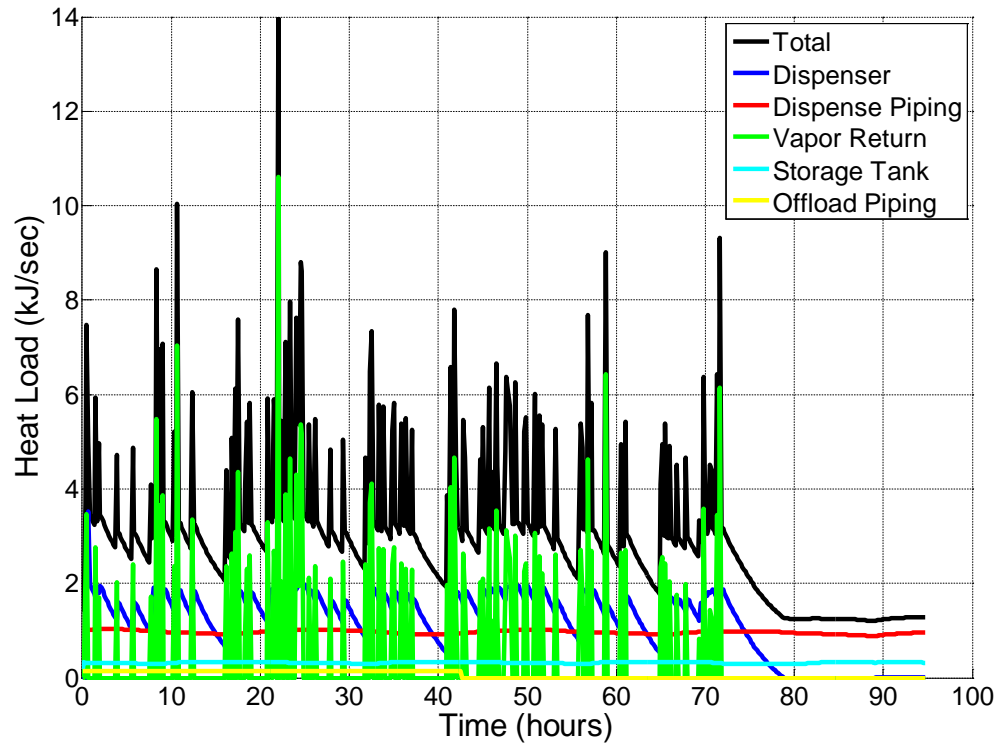

Figure C-58: Audit 1 Segment 3 Simulation Heat Transfer 


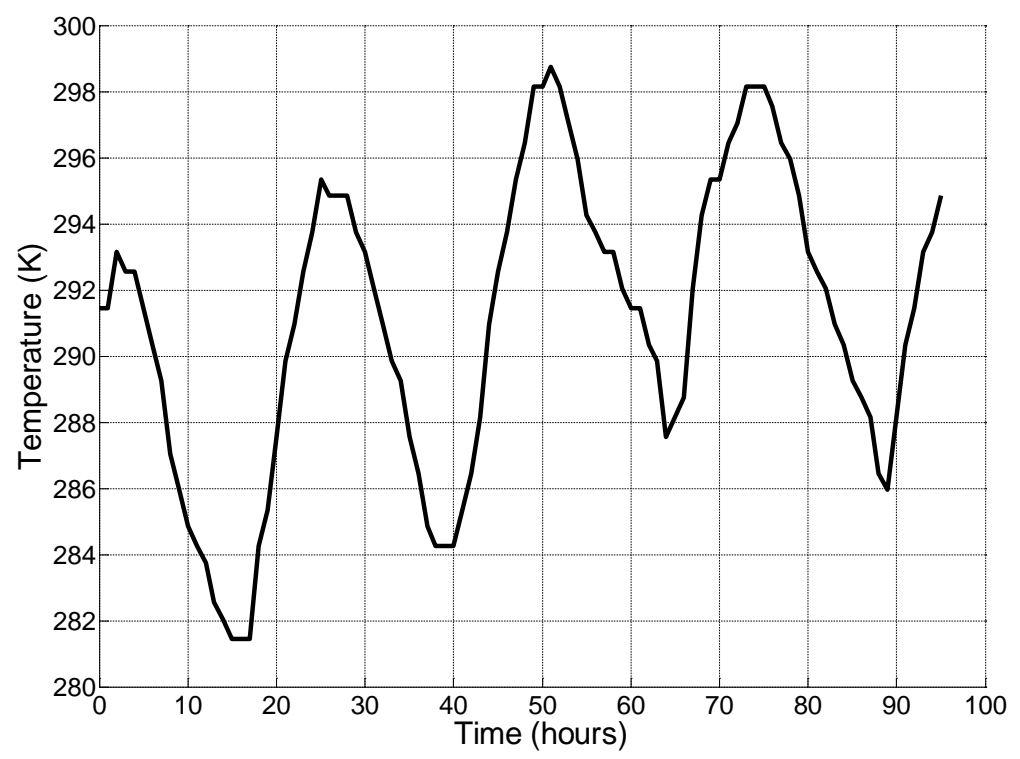

Figure C-59: Audit 1 Segment 3 Ambient Temperature
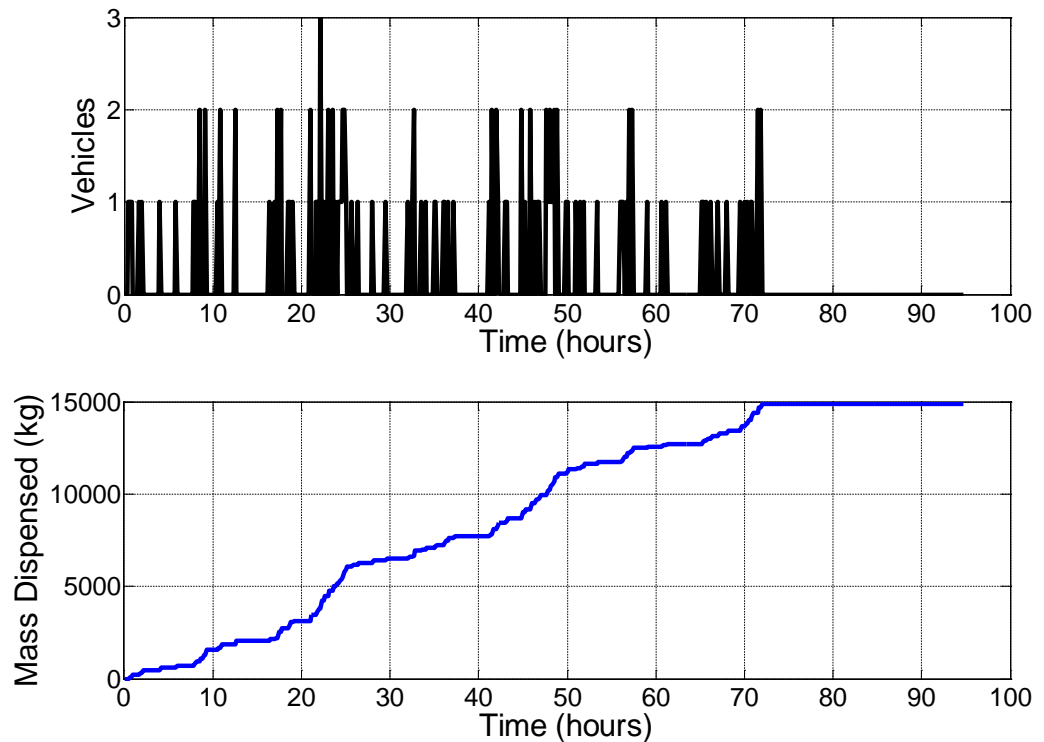

Figure C-60: Audit 1 Segment 3 Simulation Fleet Arrival Times and Mass Dispensed 


\section{$\underline{\text { Audit } 1 \text { Segment } 4}$}

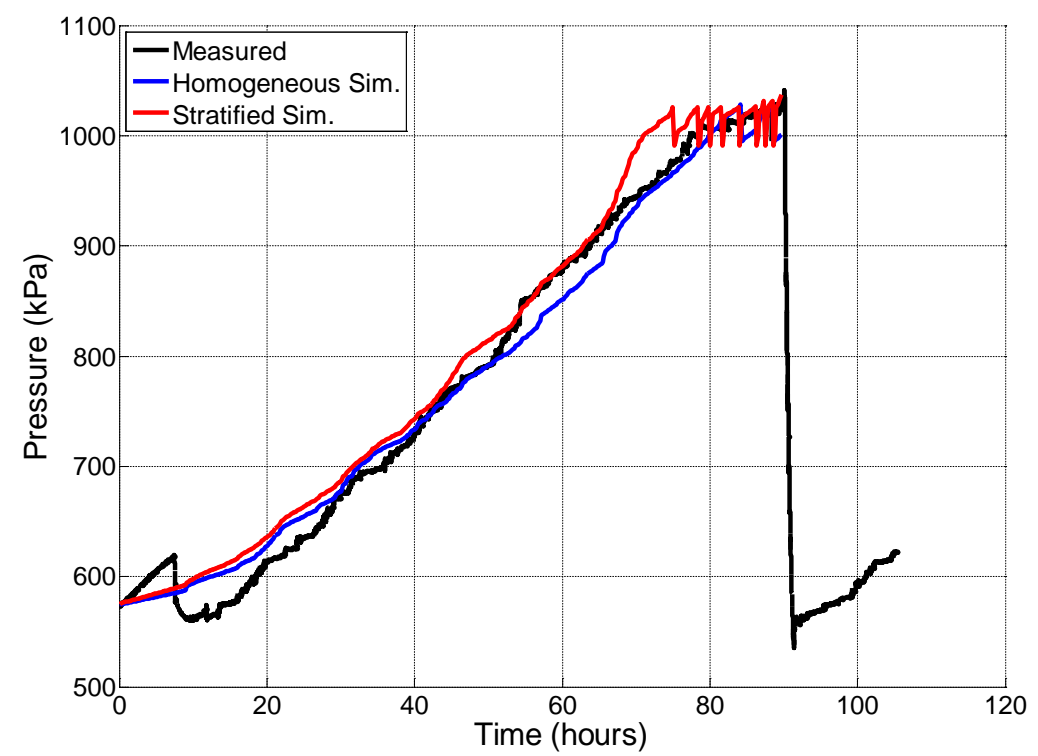

Figure C-61: Audit 1 Segment 4 Simulation Pressure Comparison

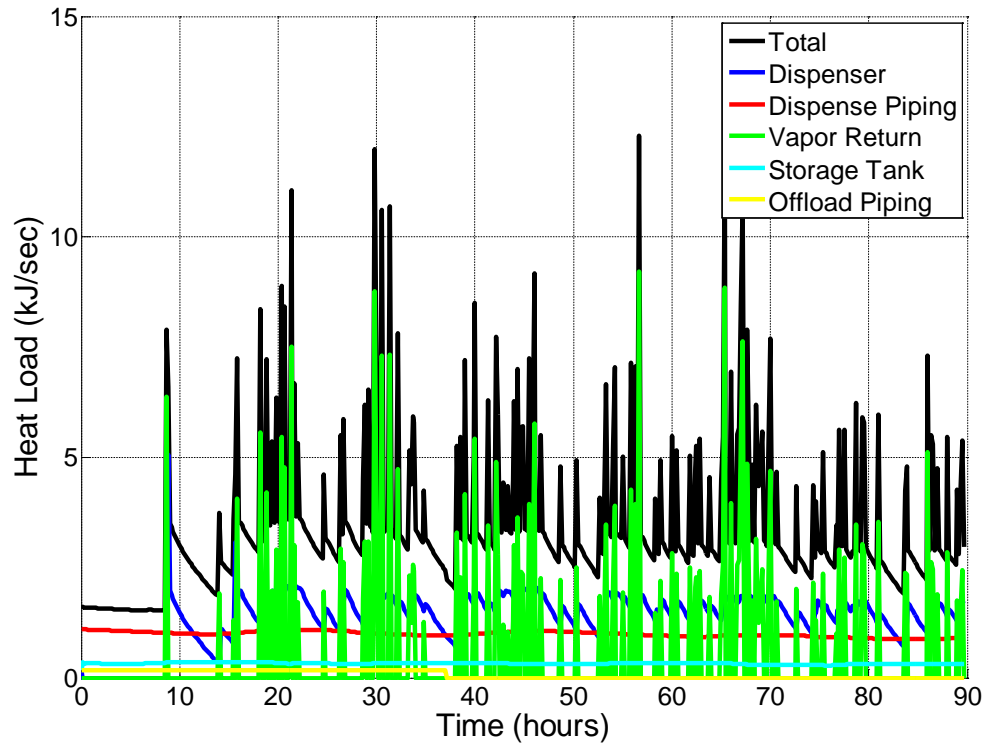

Figure C-62: Audit 1 Segment 4 Simulation Heat Transfer 


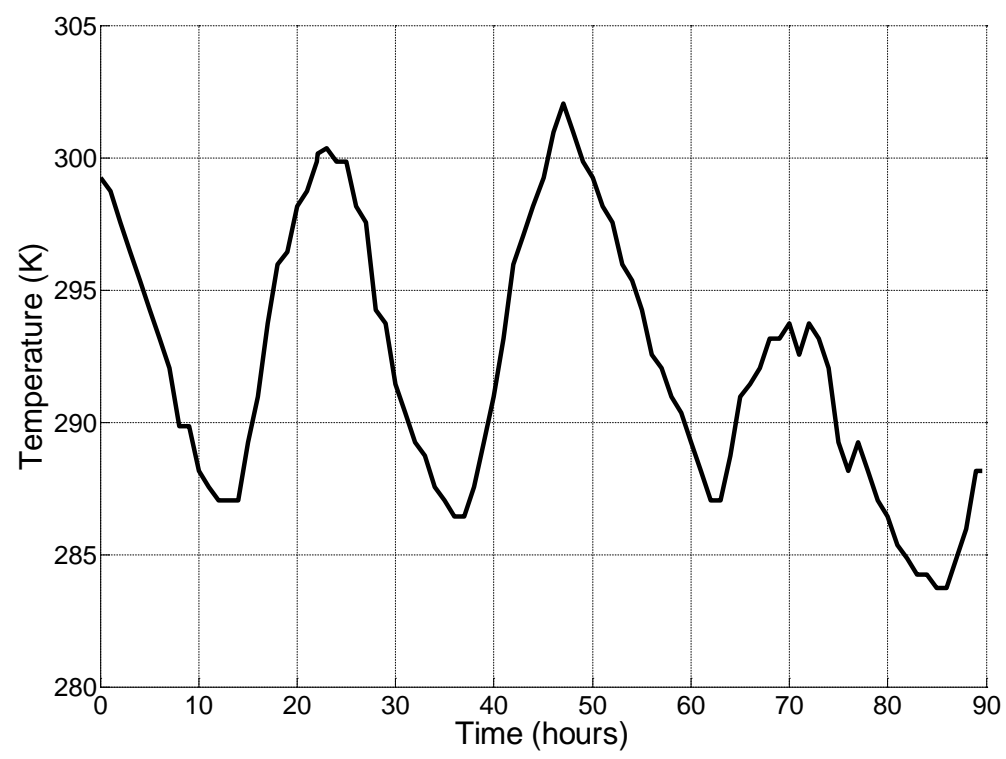

Figure C-63: Audit 1 Segment 4 Ambient Temperature
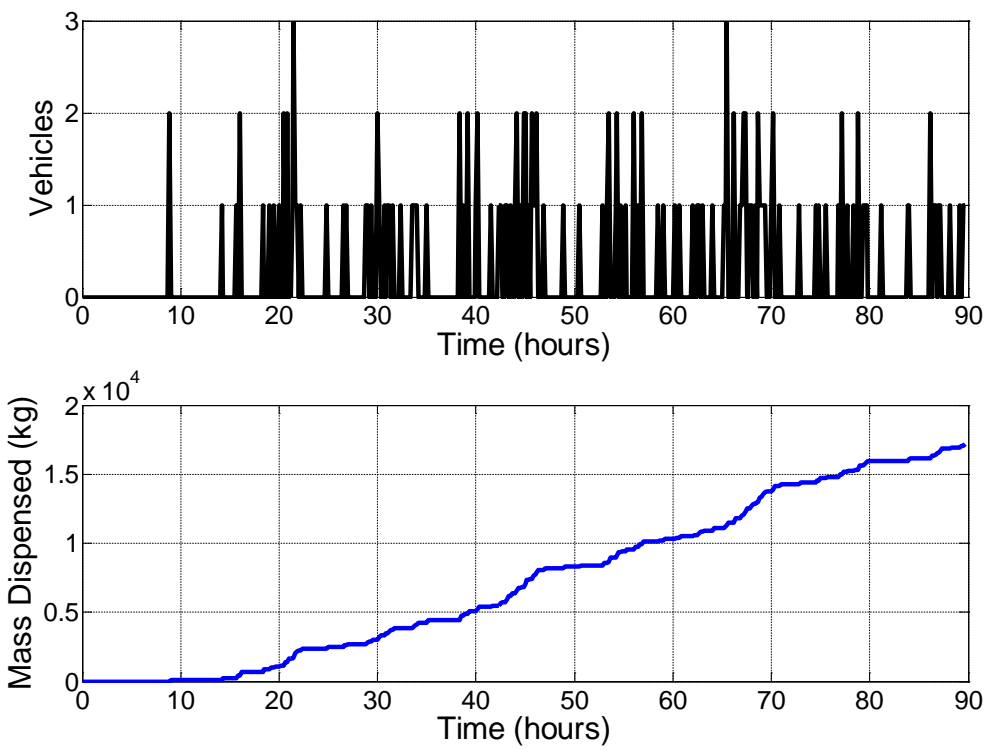

Figure C-64: Audit 1 Segment 4 Simulation Fleet Arrival Times and Mass Dispensed 


\section{$\underline{\text { Audit } 1 \text { Segment } 5}$}

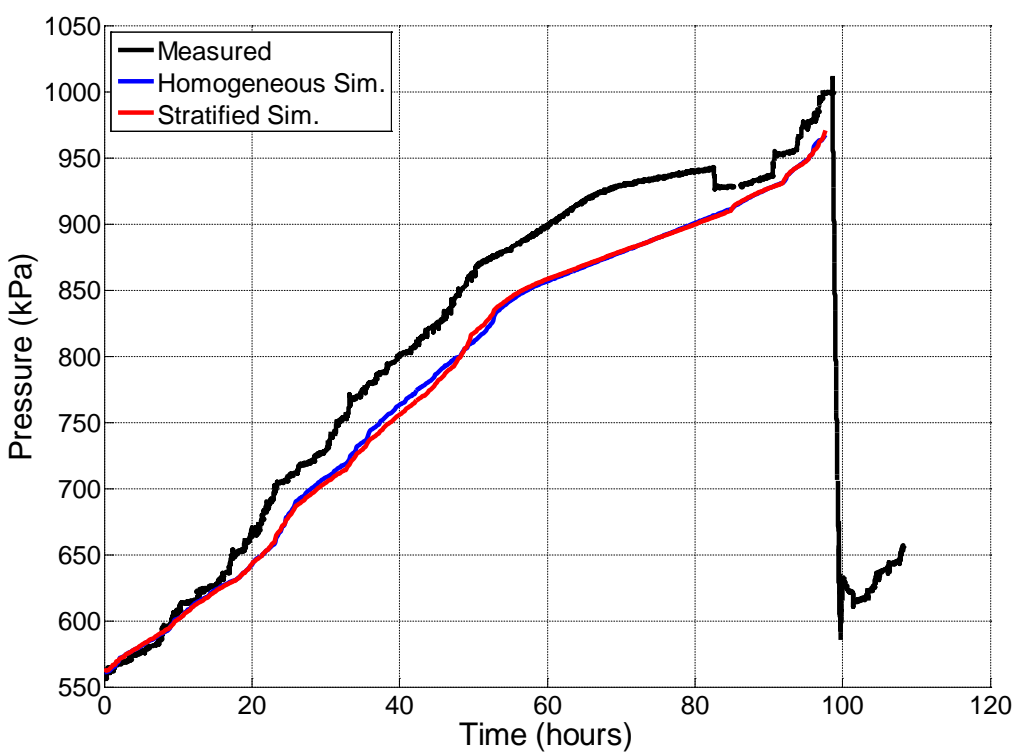

Figure C-65: Audit 1 Segment 5 Simulation Pressure Comparison

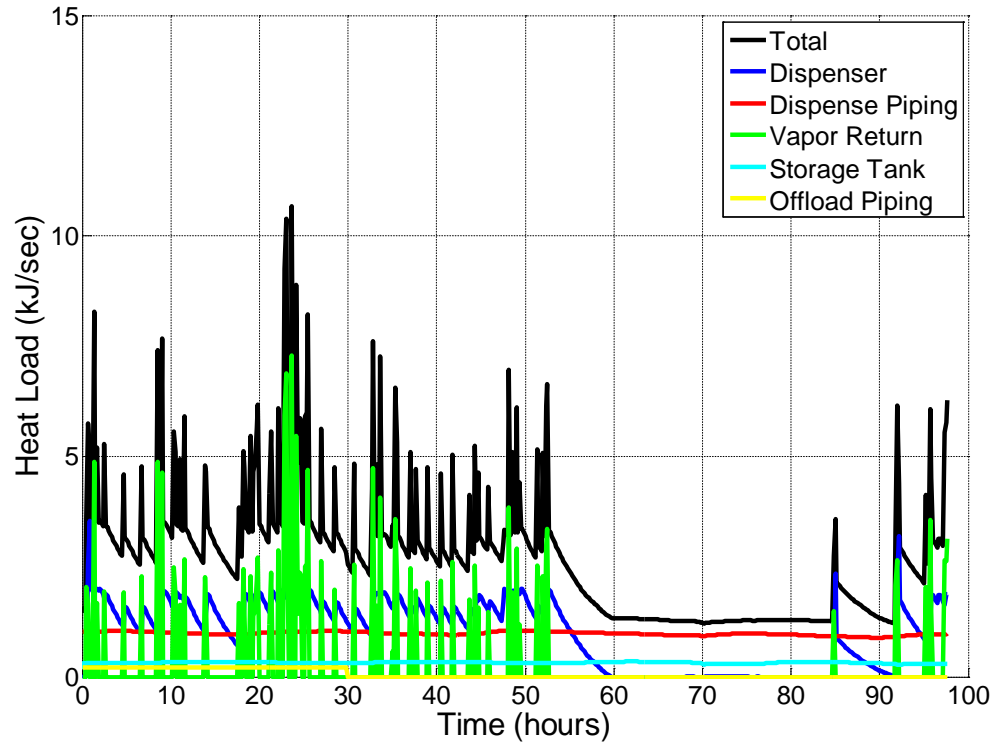

Figure C-66: Audit 1 Segment 5 Simulation Heat Transfer 


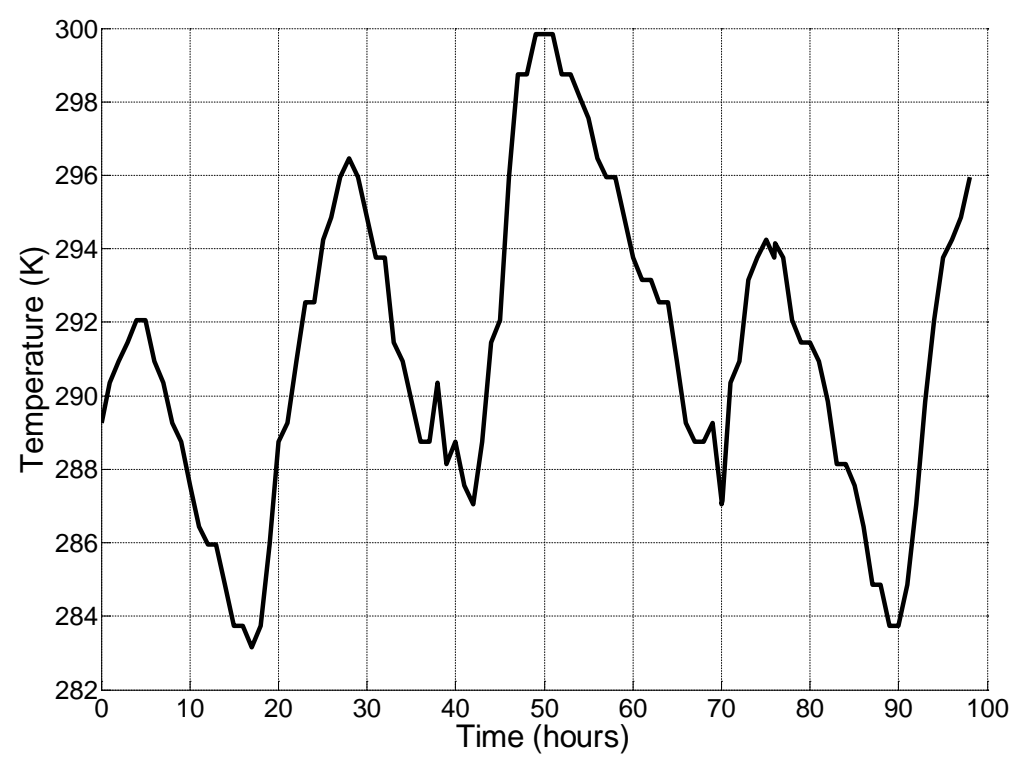

Figure C-67: Audit 1 Segment 5 Ambient Temperature
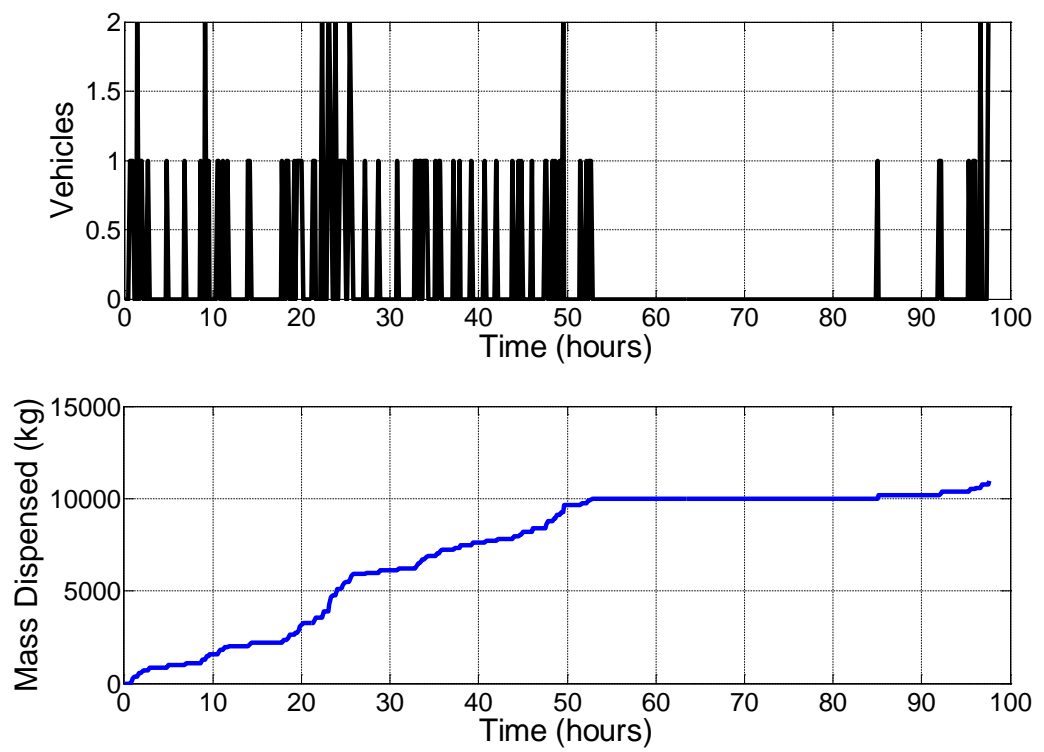

Figure C-68: Audit 1 Segment 5 Simulation Fleet Arrival Times and Mass Dispensed 


\section{$\underline{\text { Audit } 1 \text { Segment } 6}$}

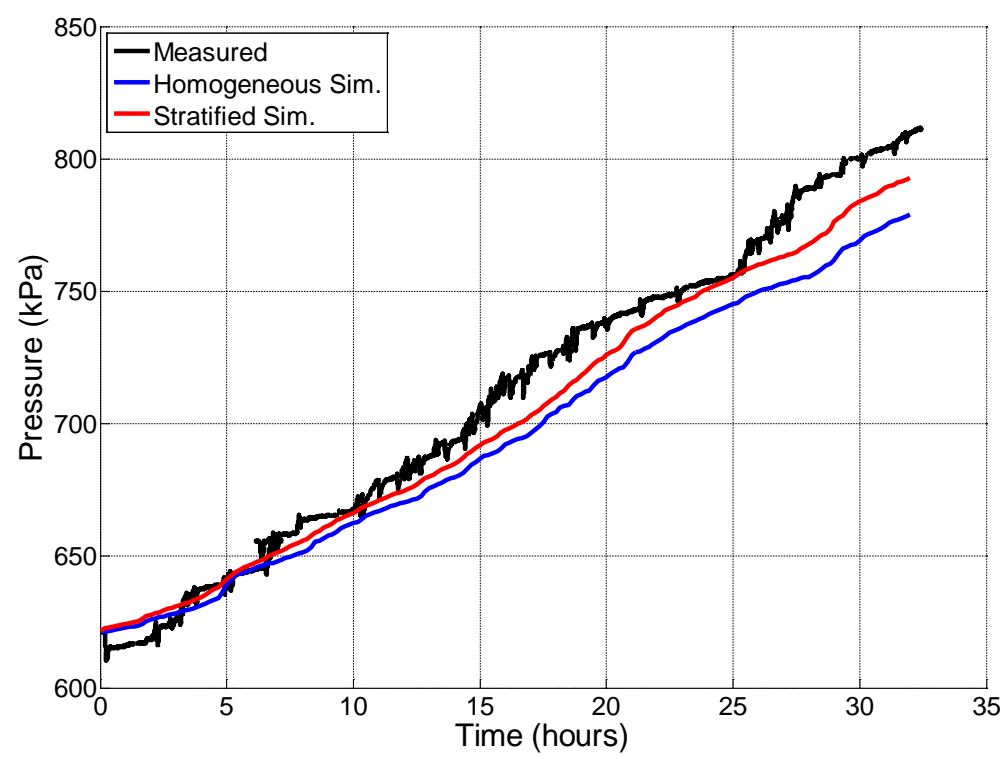

Figure C-69: Audit 1 Segment 6 Simulation Pressure Comparison

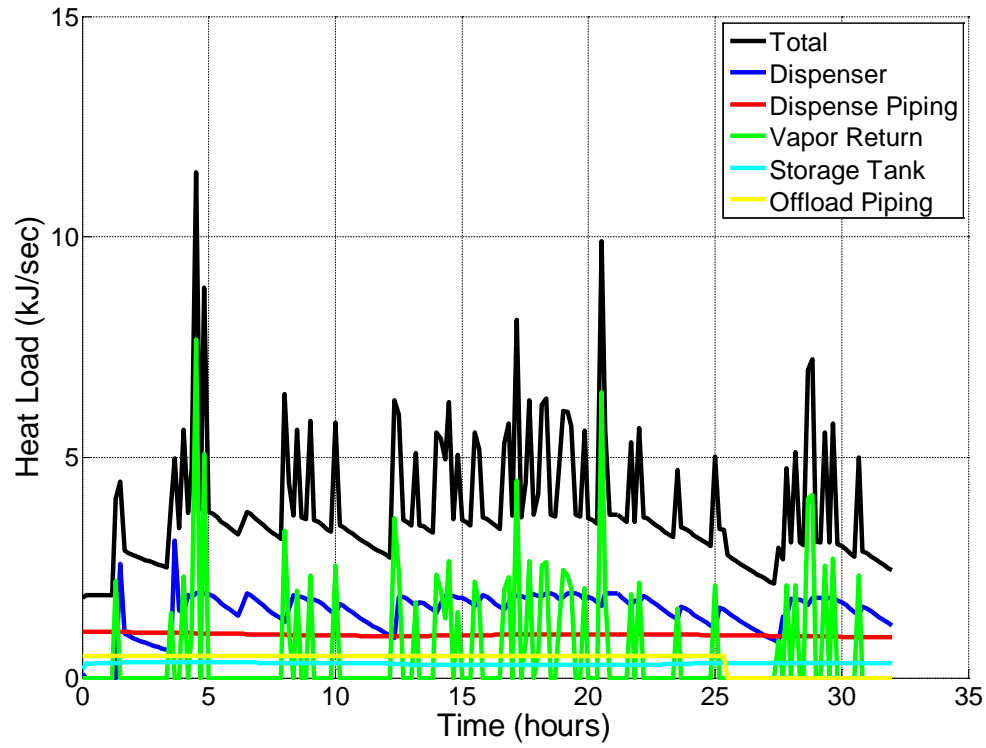

Figure C-70: Audit 1 Segment 6 Simulation Heat Transfer 


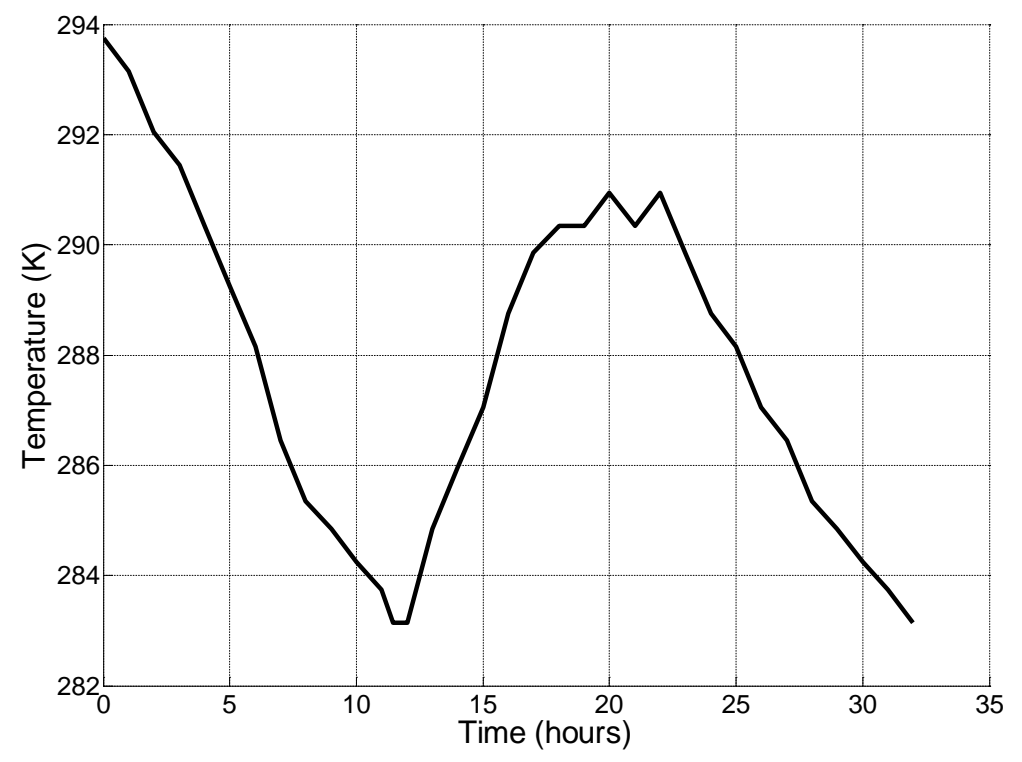

Figure C-71: Audit 1 Segment 6 Ambient Temperature
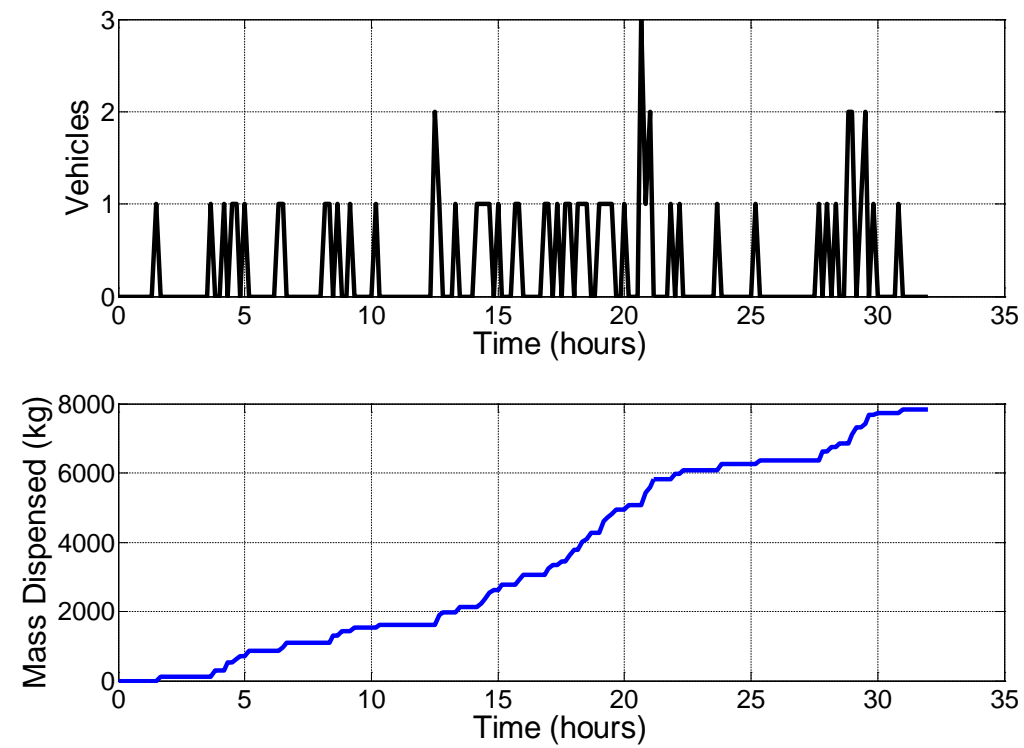

Figure C-72: Audit 1 Segment 6 Simulation Fleet Arrival Times and Mass Dispensed 


\section{Audit 2 Segment 1}

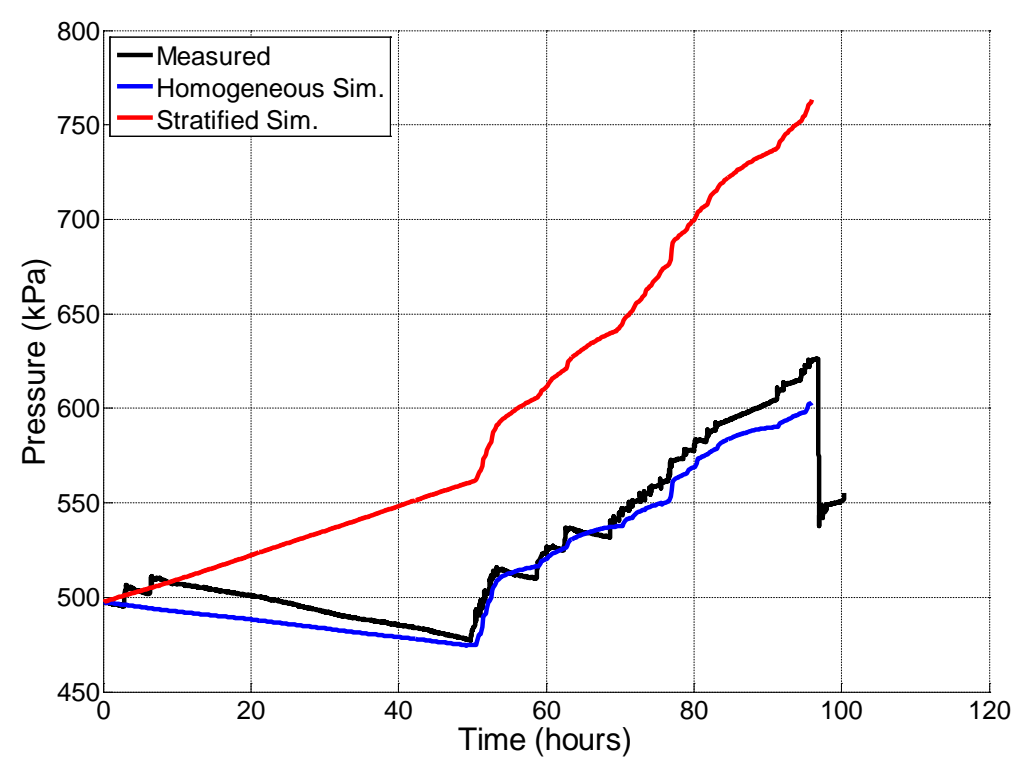

Figure C-73: Audit 2 Segment 1 Simulation Pressure Comparison

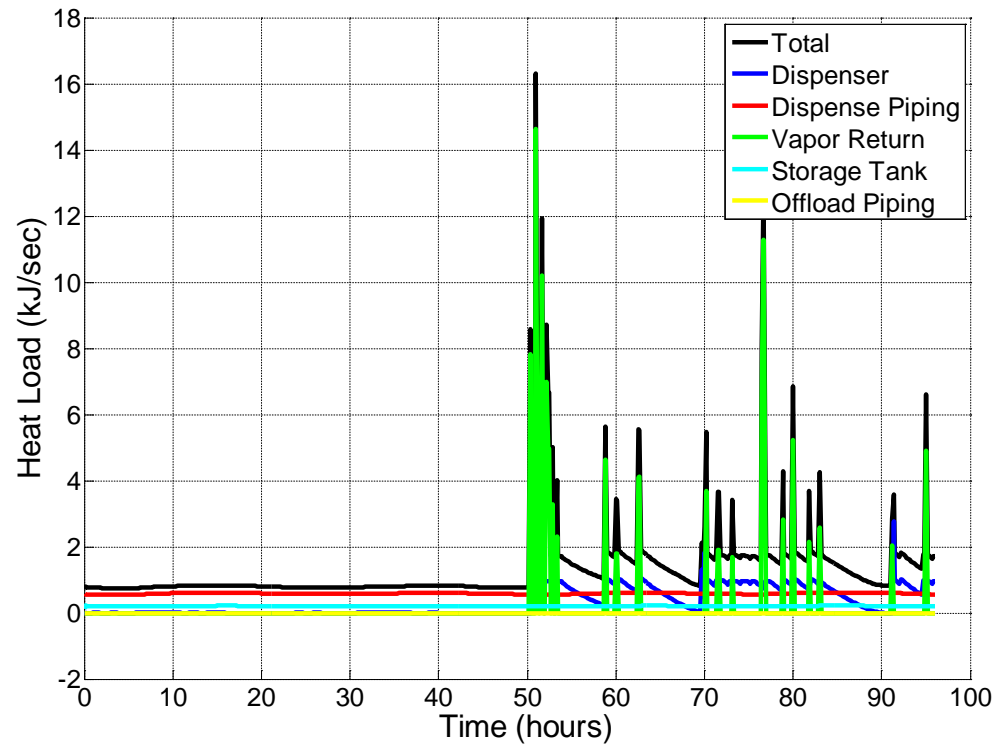

Figure C-74: Audit 2 Segment 1 Simulation Heat Transfer 


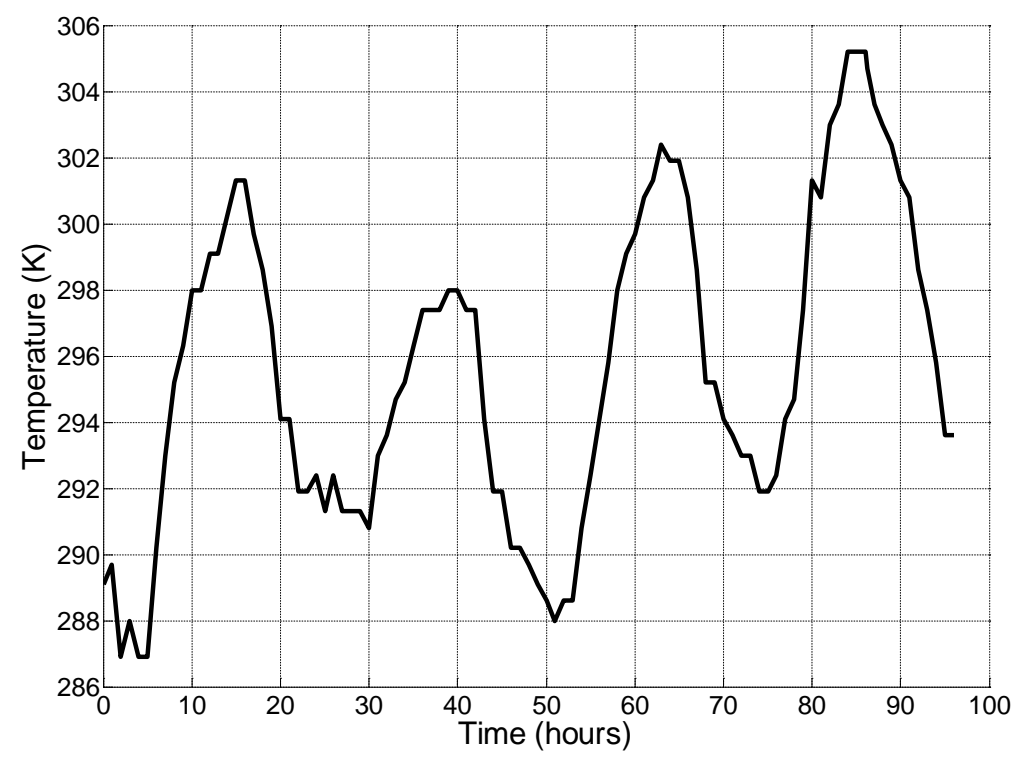

Figure C-75: Audit 2 Segment 1 Ambient Temperature
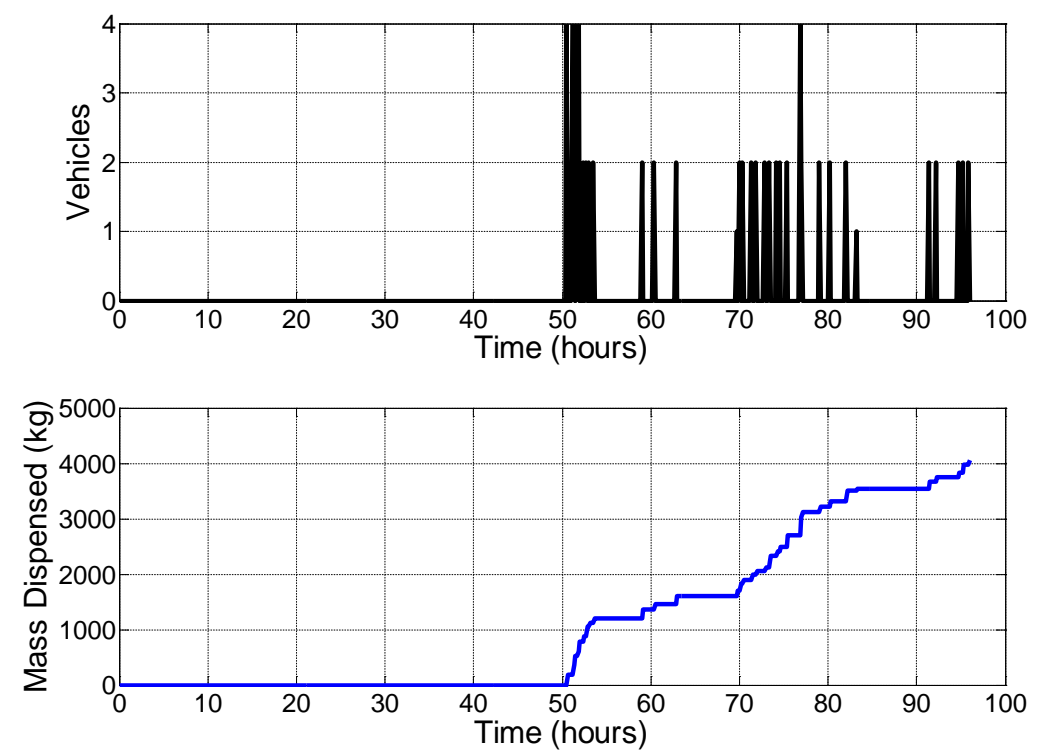

Figure C-76: Audit 2 Segment 1 Simulation Fleet Arrival Times and Mass Dispensed 


\section{$\underline{\text { Audit } 2 \text { Segment } 2}$}

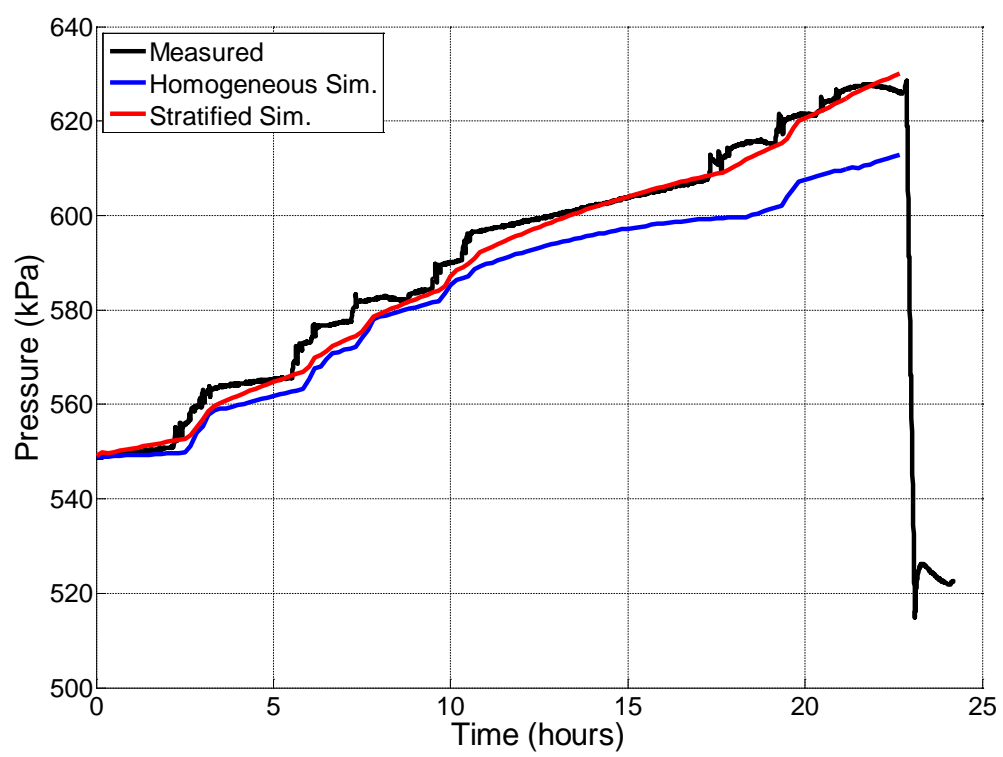

Figure C-77: Audit 2 Segment 2 Simulation Pressure Comparison

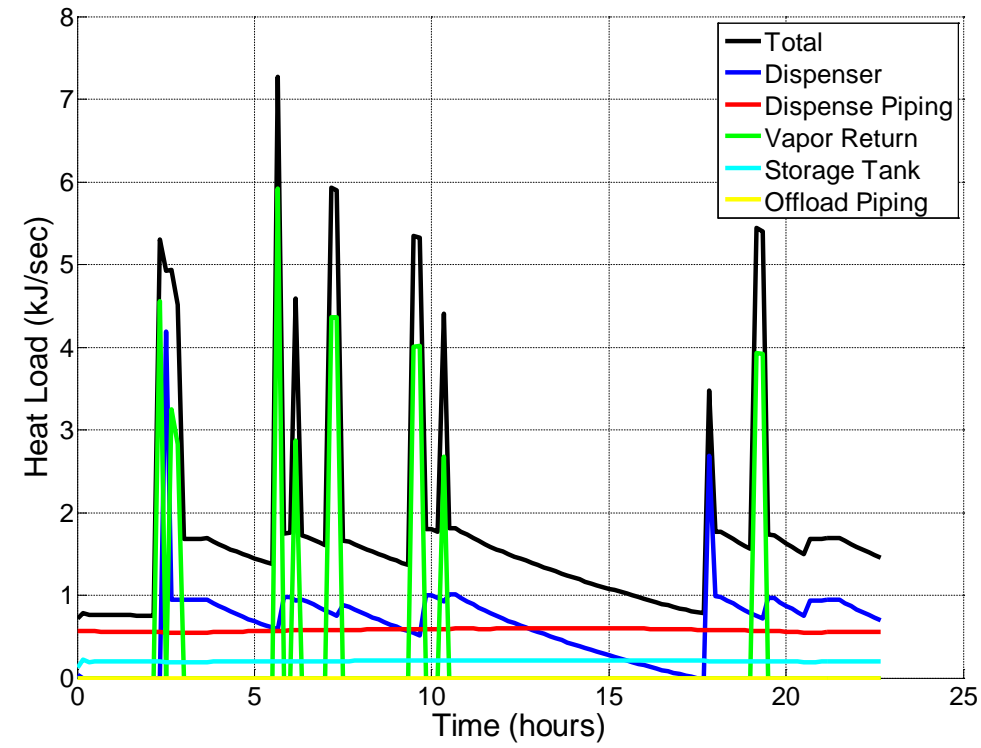

Figure C-78: Audit 2 Segment 2 Simulation Heat Transfer 


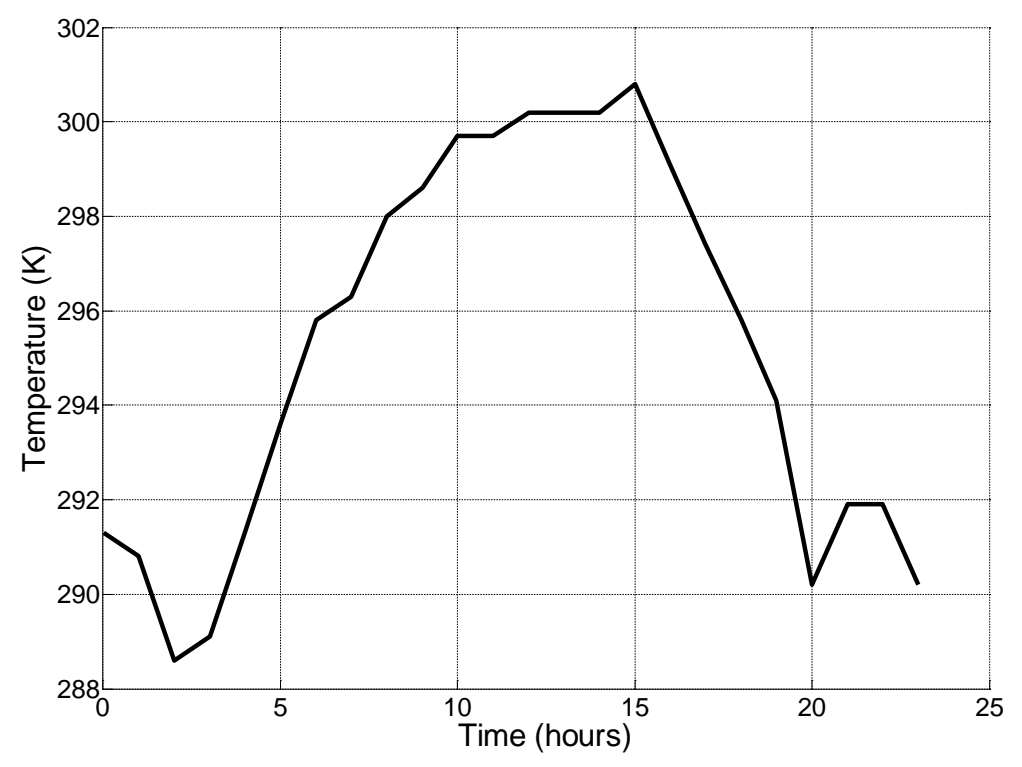

Figure C-79: Audit 2 Segment 2 Ambient Temperature
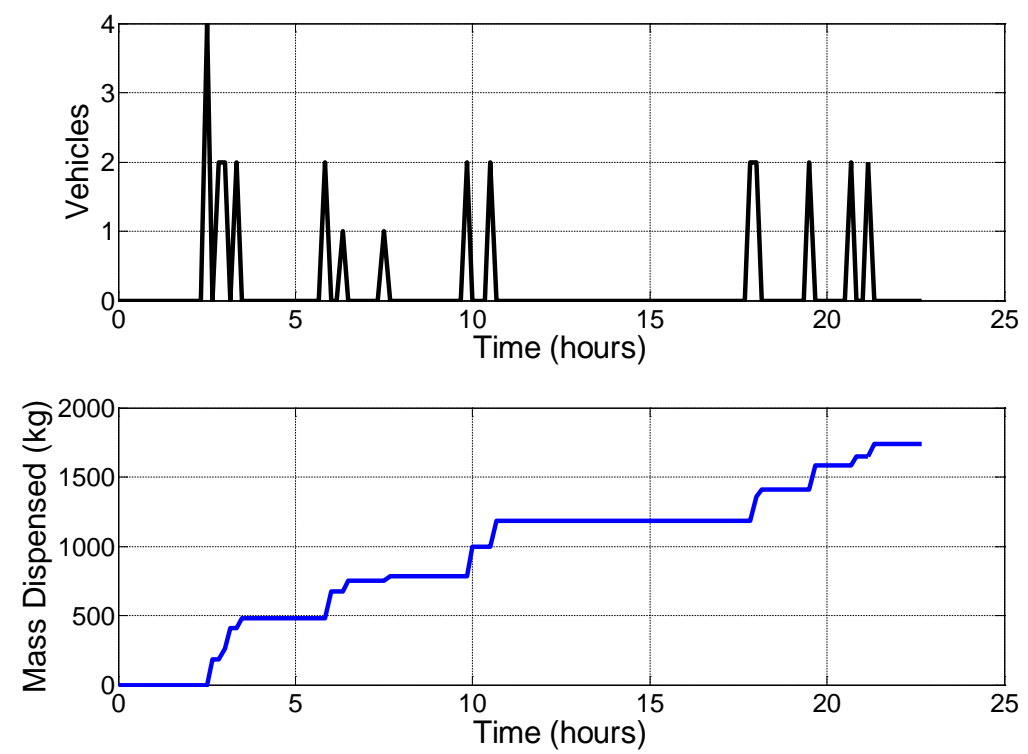

Figure C-80: Audit 2 Segment 2 Simulation Fleet Arrival Times and Mass Dispensed 


\section{$\underline{\text { Audit } 2 \text { Segment } 3}$}

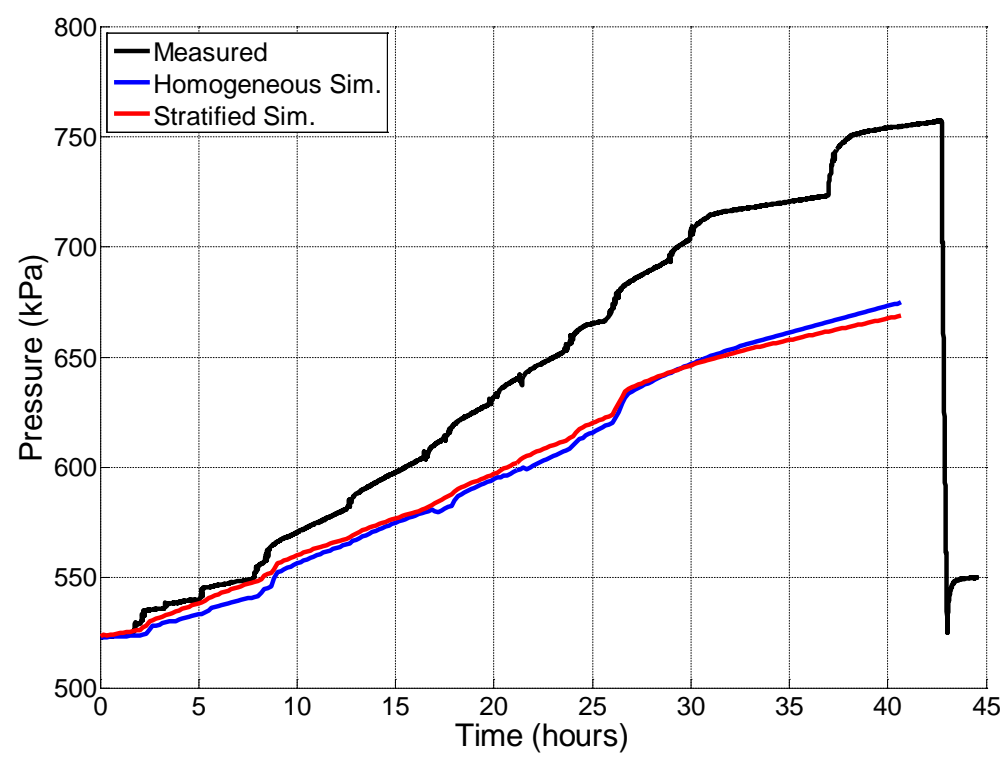

Figure C-81: Audit 2 Segment 3 Simulation Pressure Comparison

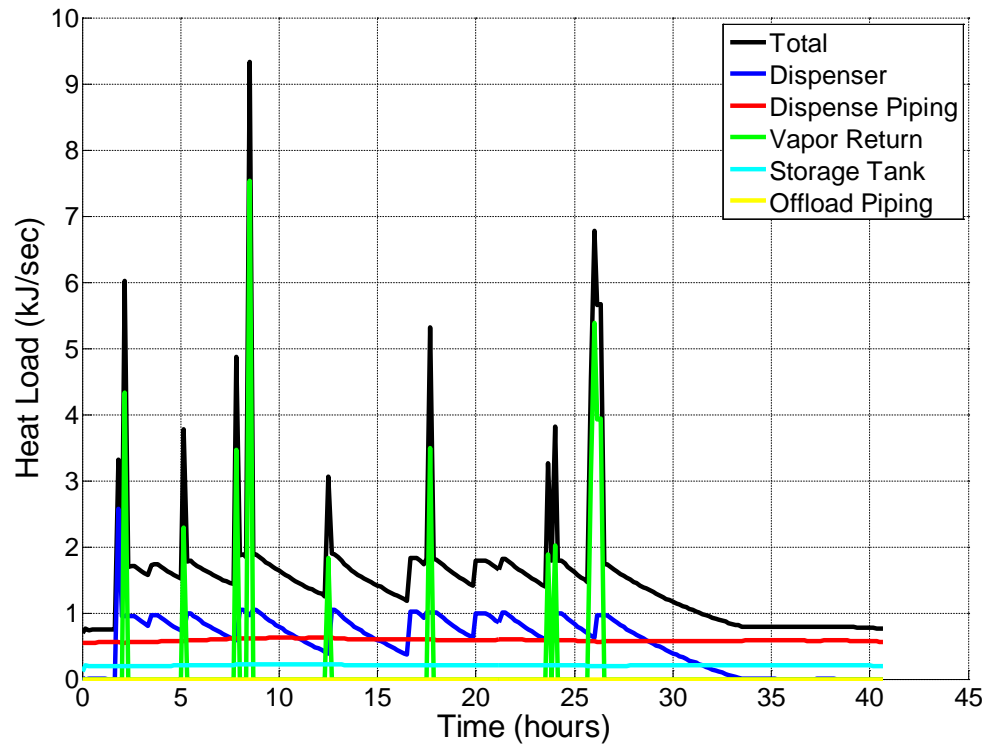

Figure C-82: Audit 2 Segment 3 Simulation Heat Transfer 

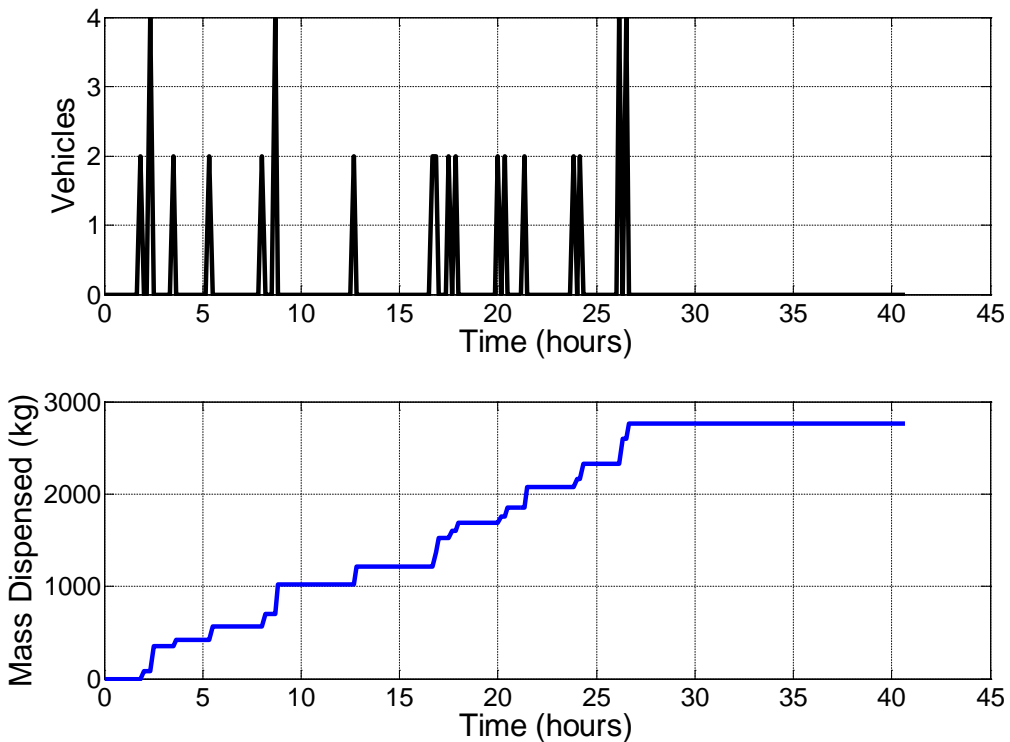

Figure C-83: Audit 2 Segment 3 Simulation Fleet Arrival Times and Mass Dispensed

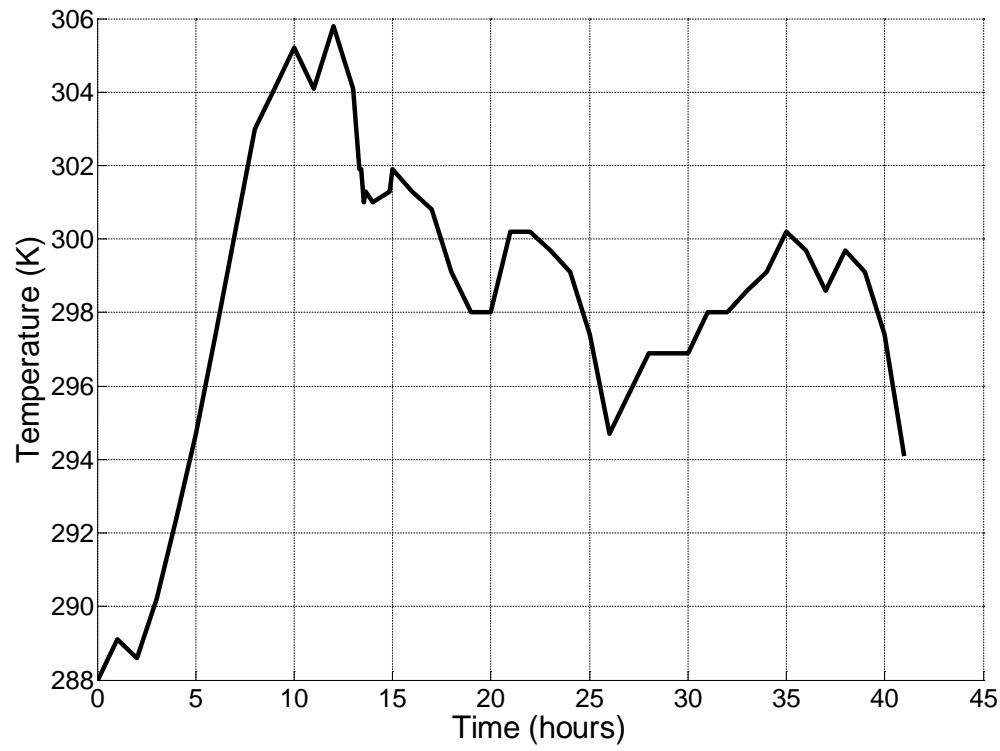

Figure C-84: Audit 2 Segment 3 Ambient Temperature 


\section{Audit 2 Segment 4}

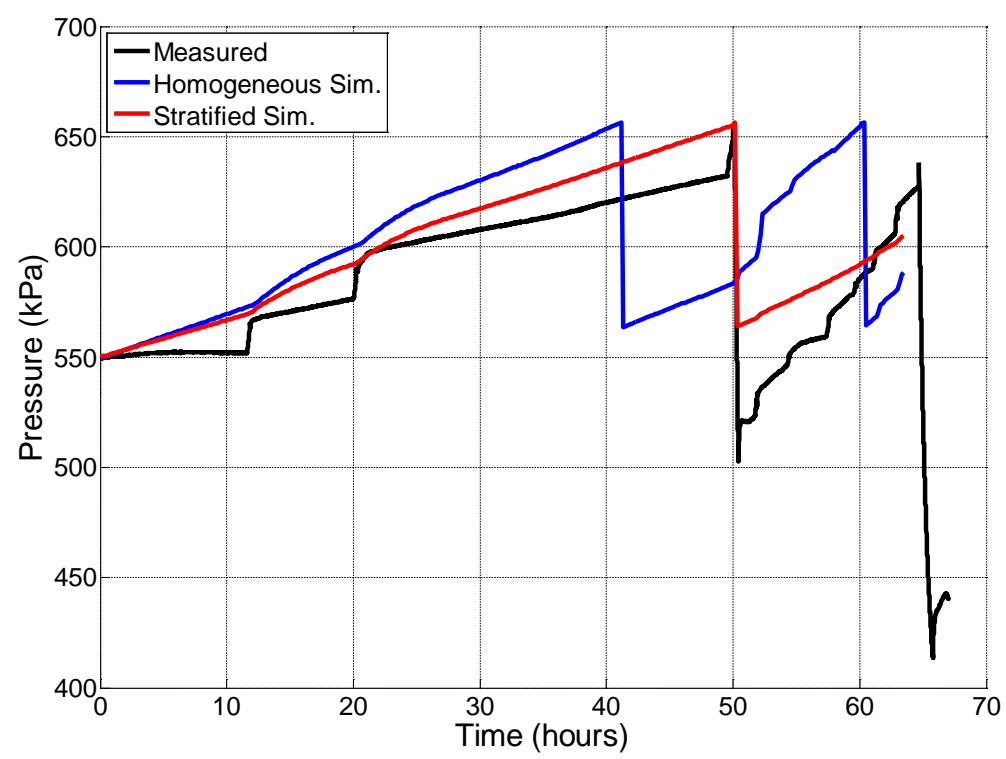

Figure C-85: Audit 2 Segment 4 Simulation Pressure Comparison

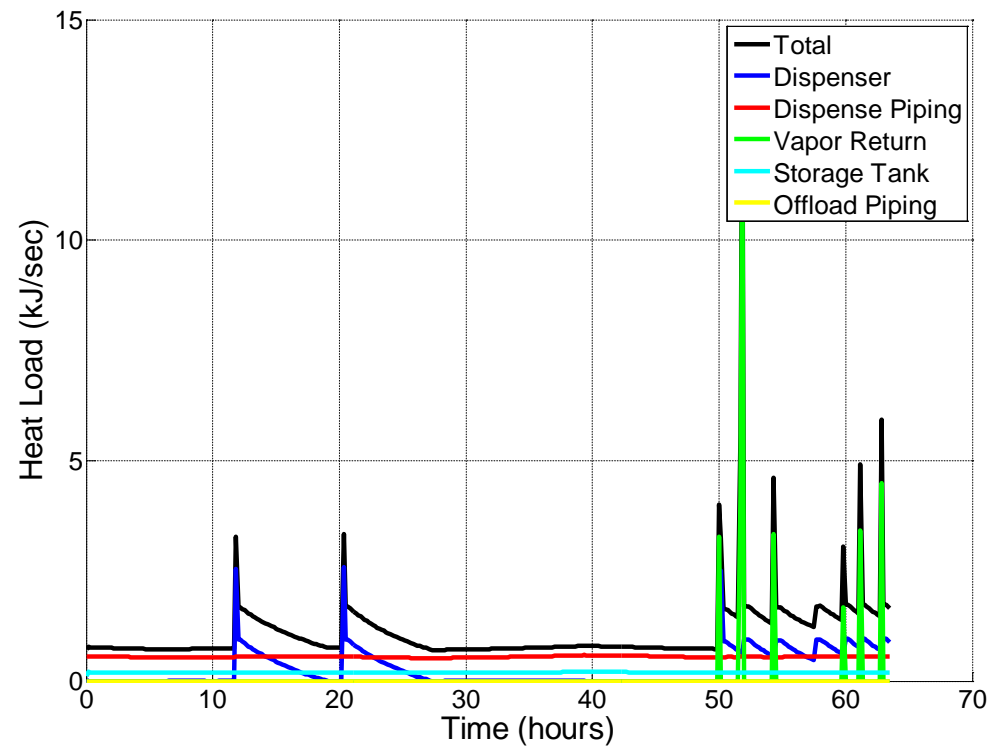

Figure C-86: Audit 2 Segment 4 Simulation Heat Transfer 


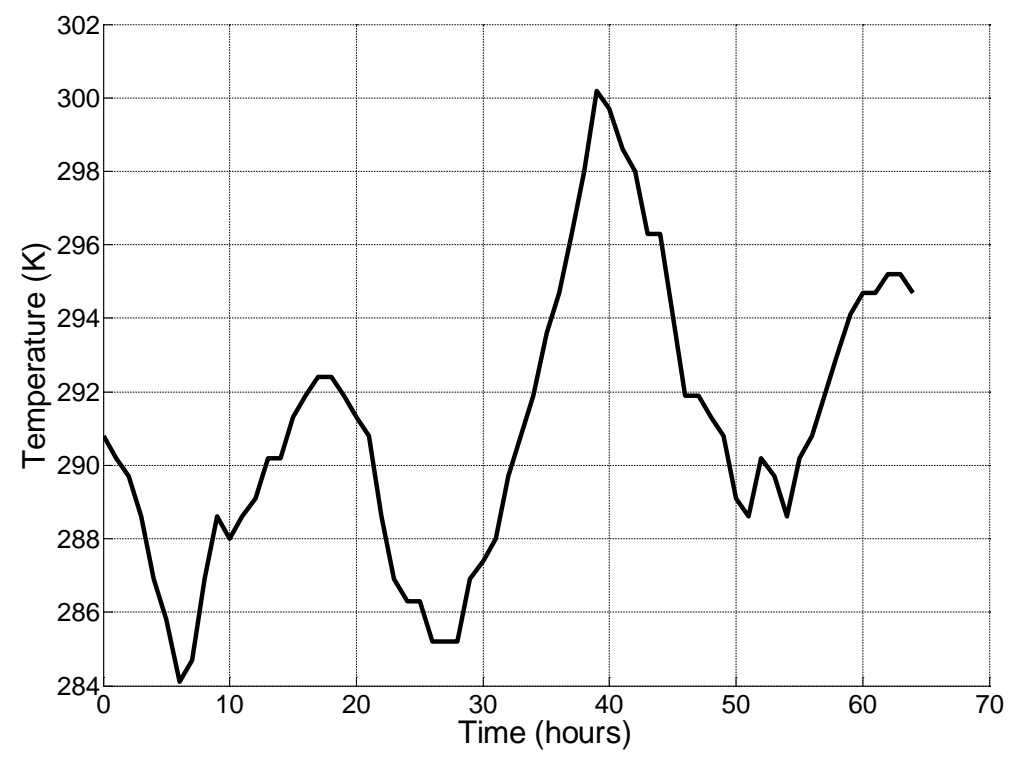

Figure C-87: Audit 2 Segment 4 Ambient Temperature
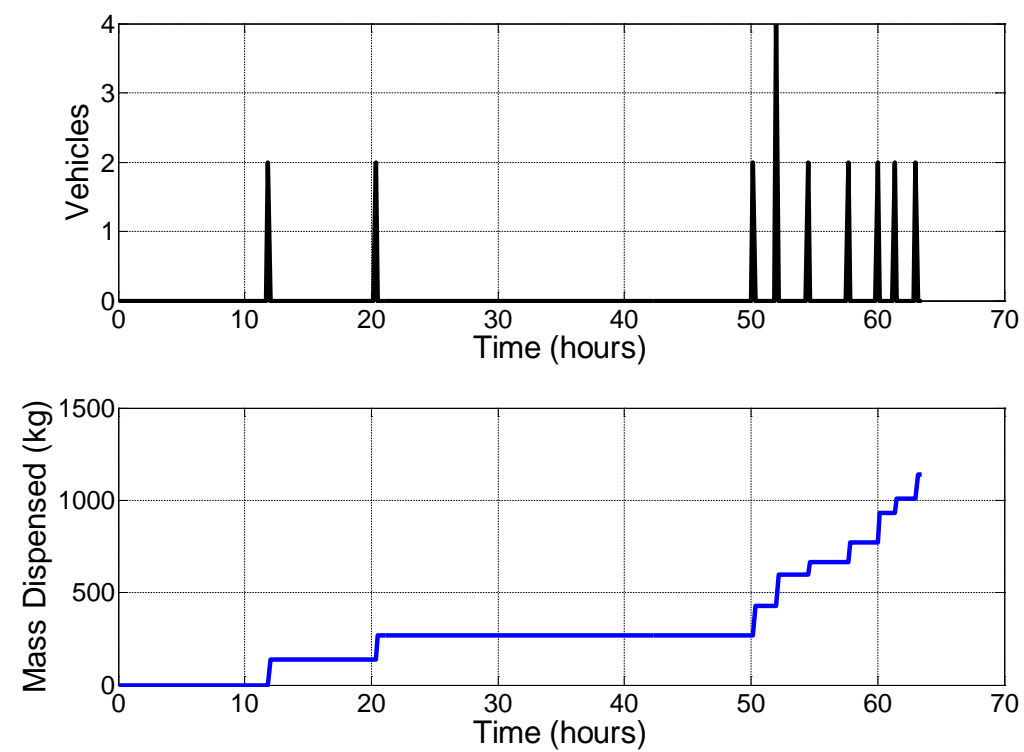

Figure C-88: Audit 2 Segment 4 Simulation Fleet Arrival Times and Mass Dispensed 


\section{Audit 2 Segment 5}

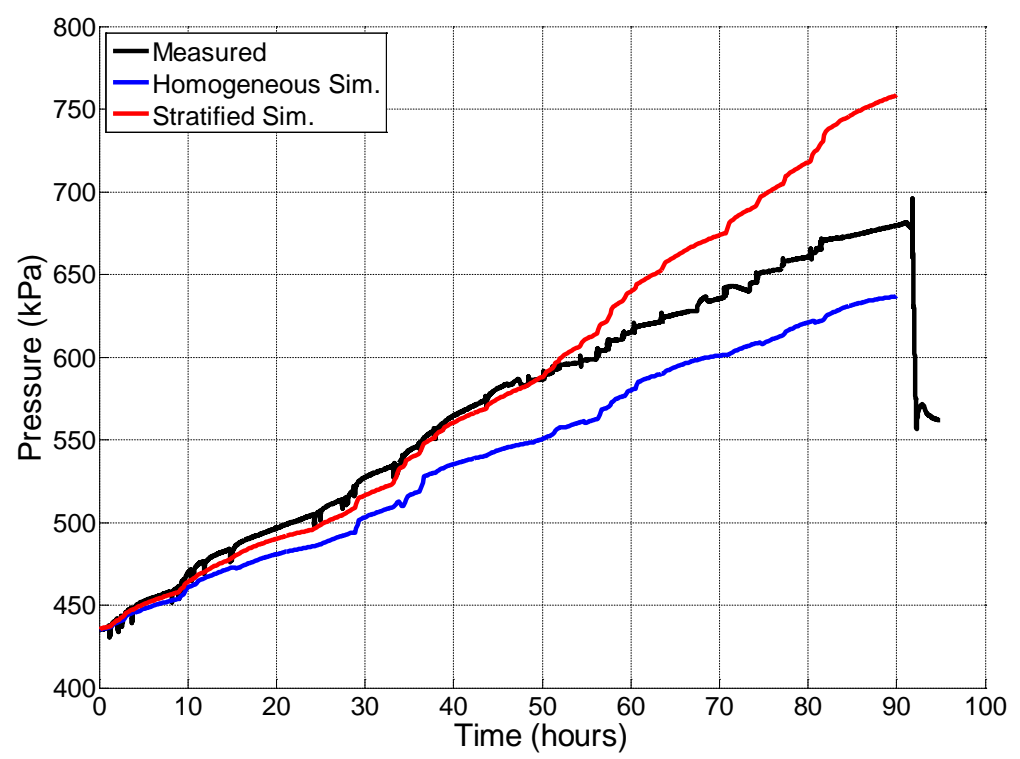

Figure C-89: Audit 2 Segment 5 Simulation Pressure Comparison

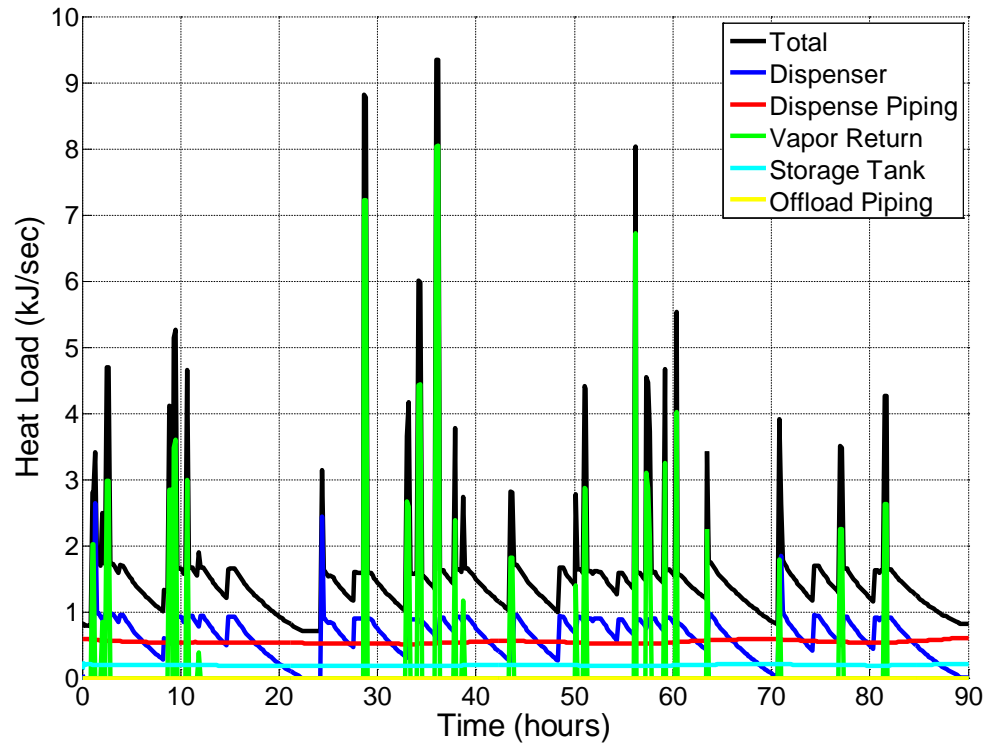

Figure C-90: Audit 2 Segment 5 Simulation Heat Transfer 


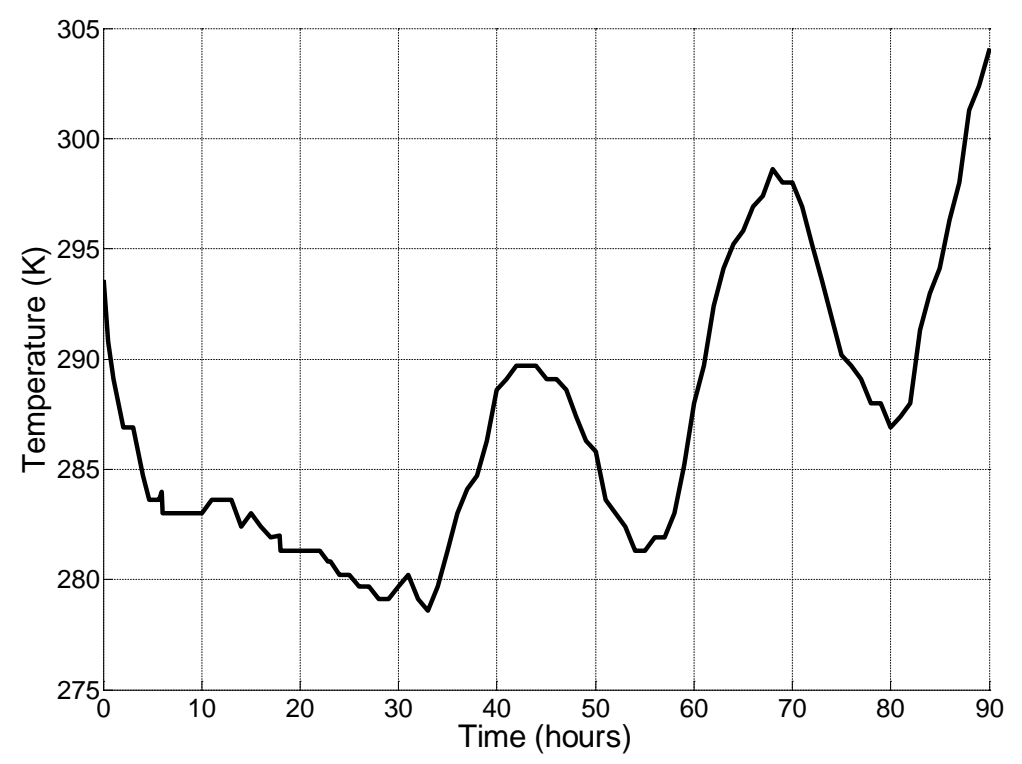

Figure C-91: Audit 2 Segment 5 Ambient Temperature
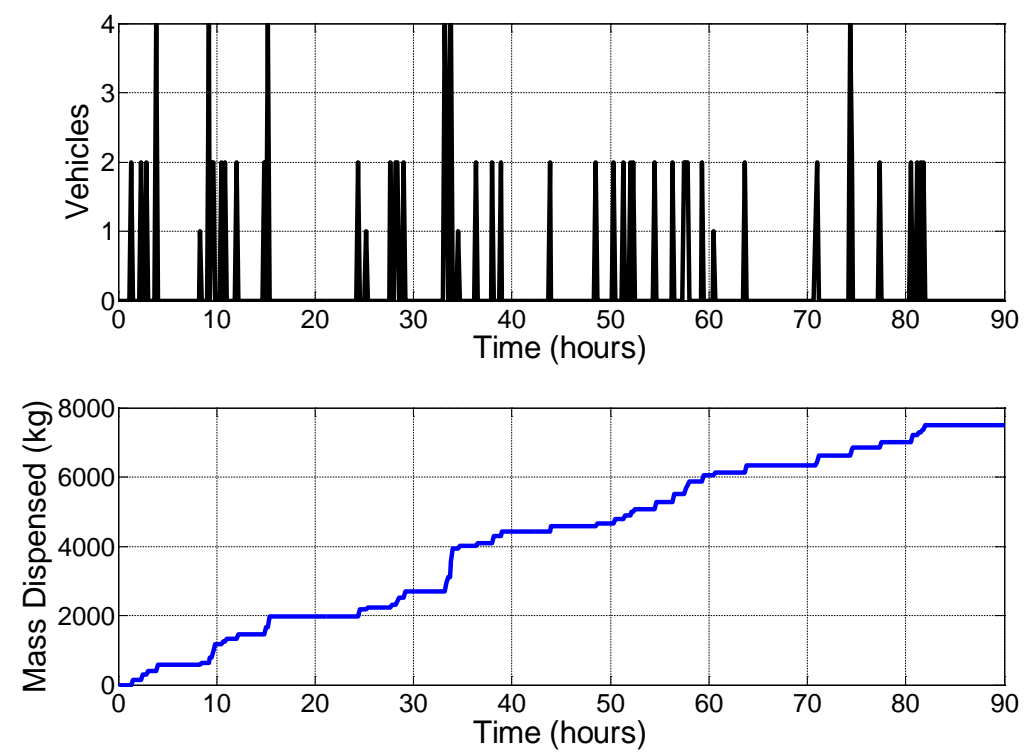

Figure C-92: Audit 2 Segment 5 Simulation Fleet Arrival Times and Mass Dispensed 


\section{Audit 2 Segment 6}

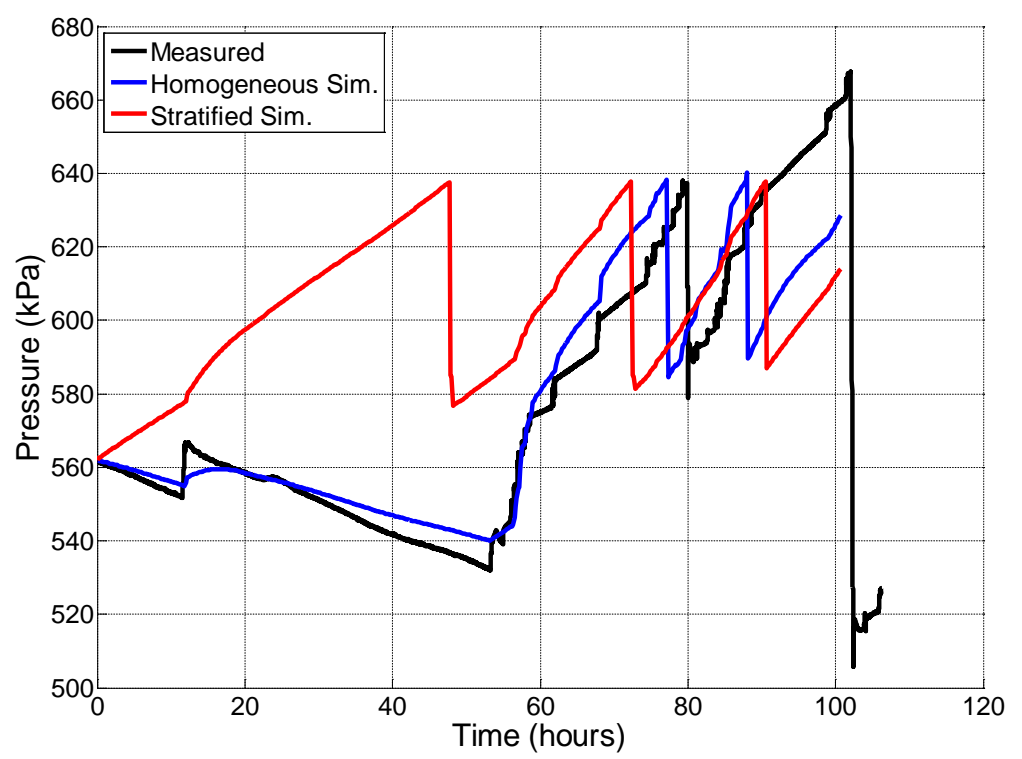

Figure C-93: Audit 2 Segment 6 Simulation Pressure Comparison

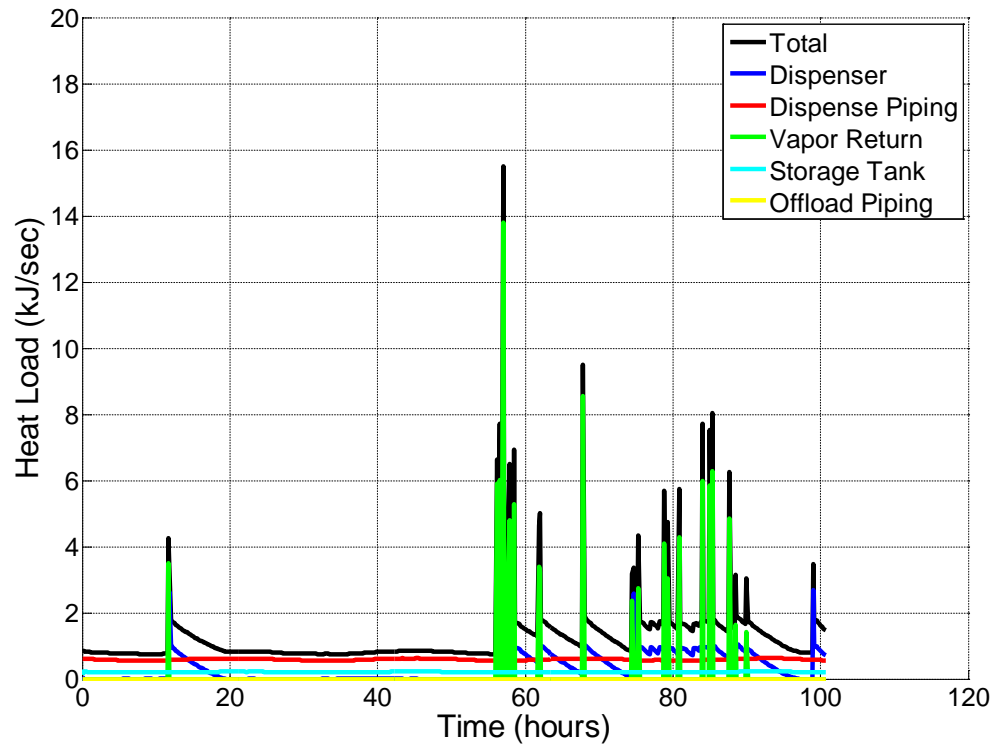

Figure C-94: Audit 2 Segment 6 Simulation Heat Transfer 

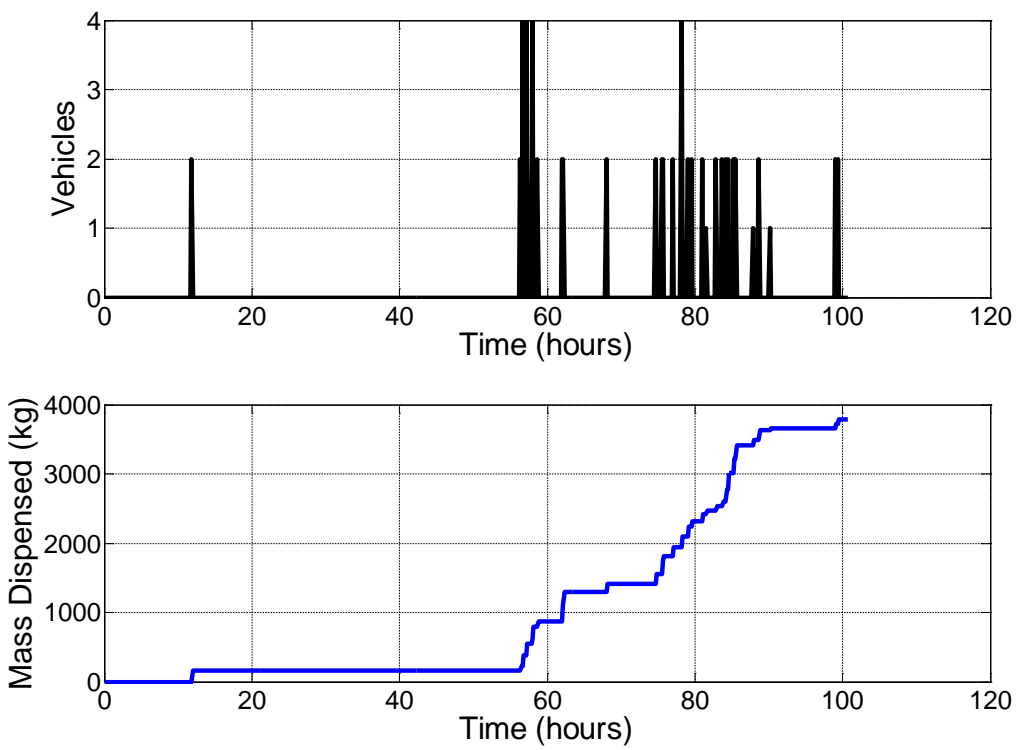

Figure C-95: Audit 2 Segment 6 Simulation Fleet Arrival Times and Mass Dispensed

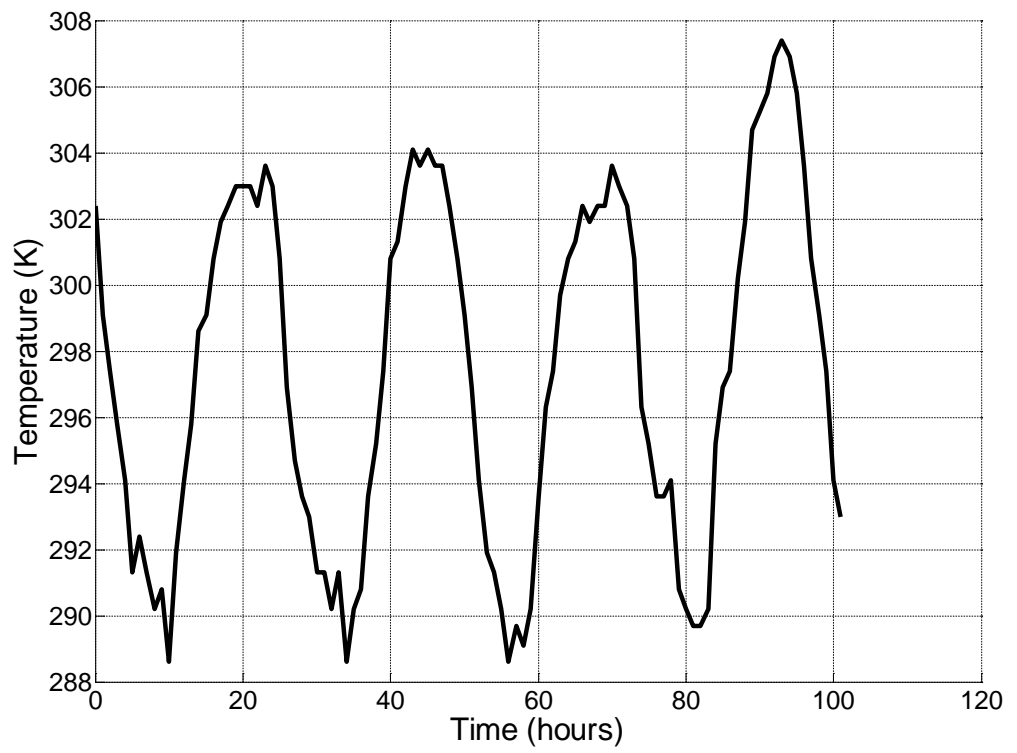

Figure C-96: Audit 2 Segment 6 Ambient Temperature 


\section{$\underline{\text { Audit } 2 \text { Segment } 7}$}

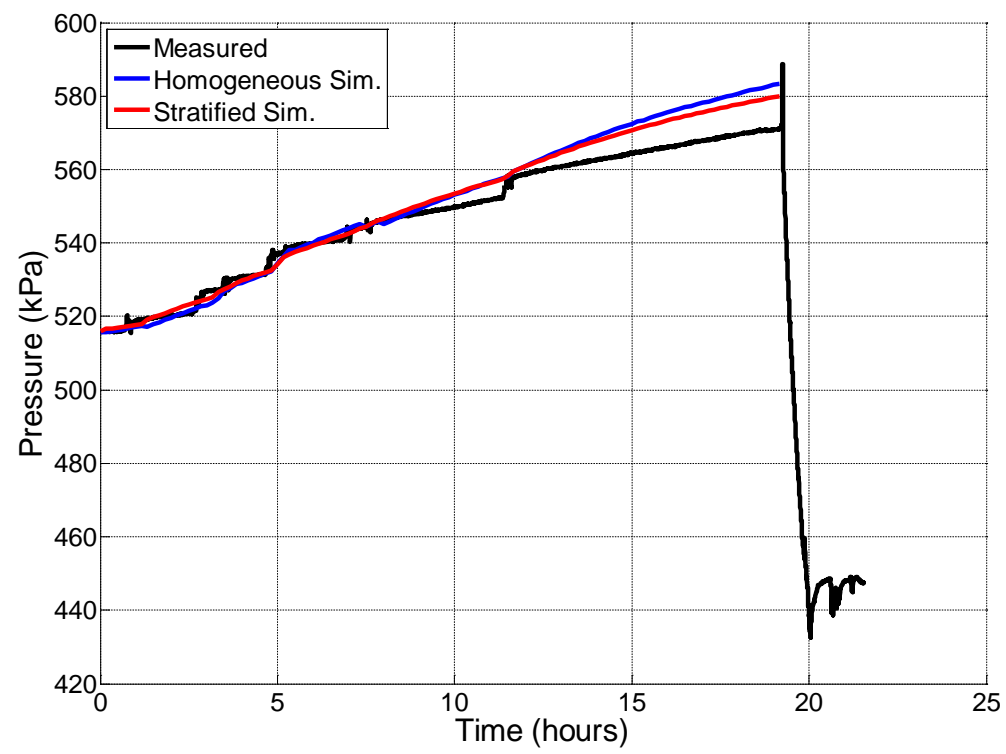

Figure C-97: Audit 2 Segment 7 Simulation Pressure Comparison

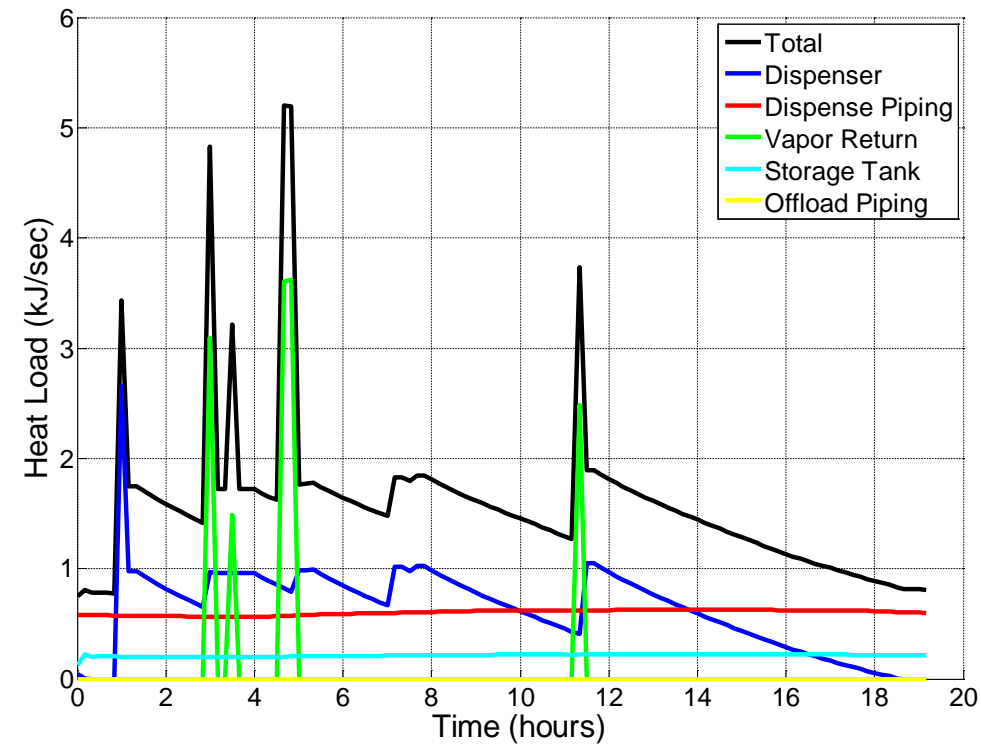

Figure C-98: Audit 2 Segment 7 Simulation Heat Transfer 

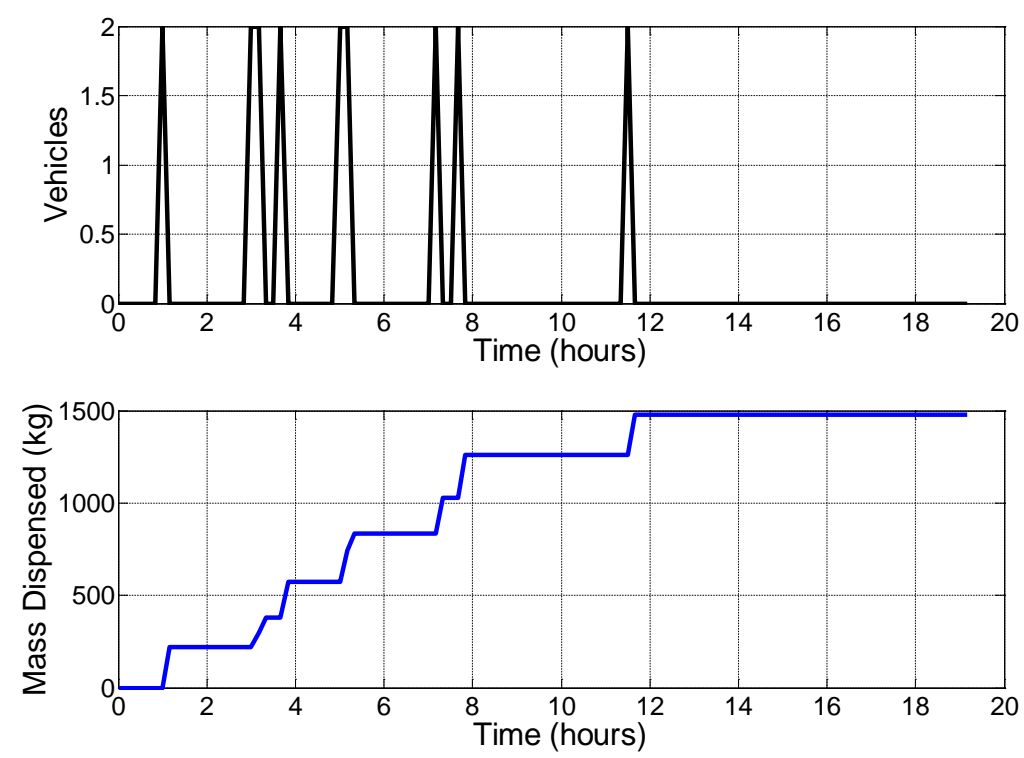

Figure C-99: Audit 2 Segment 7 Simulation Fleet Arrival Times and Mass Dispensed

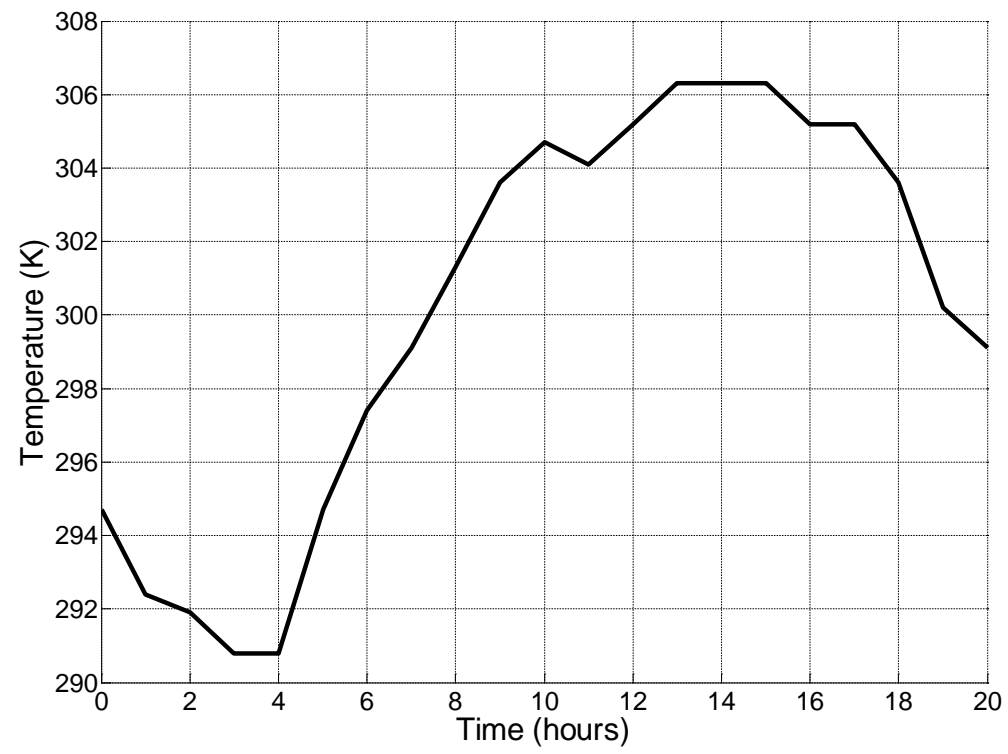

Figure C-100: Audit 2 Segment 7 Ambient Temperature 


\section{Audit 2 Segment 8}

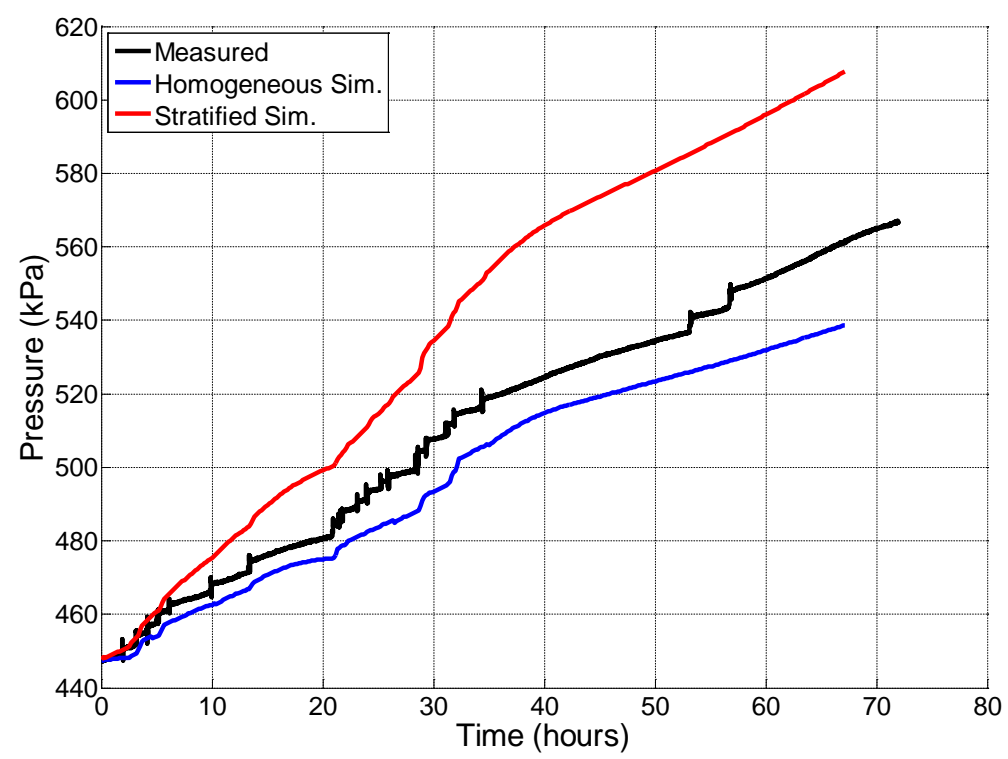

Figure C-101: Audit 2 Segment 8 Simulation Pressure Comparison

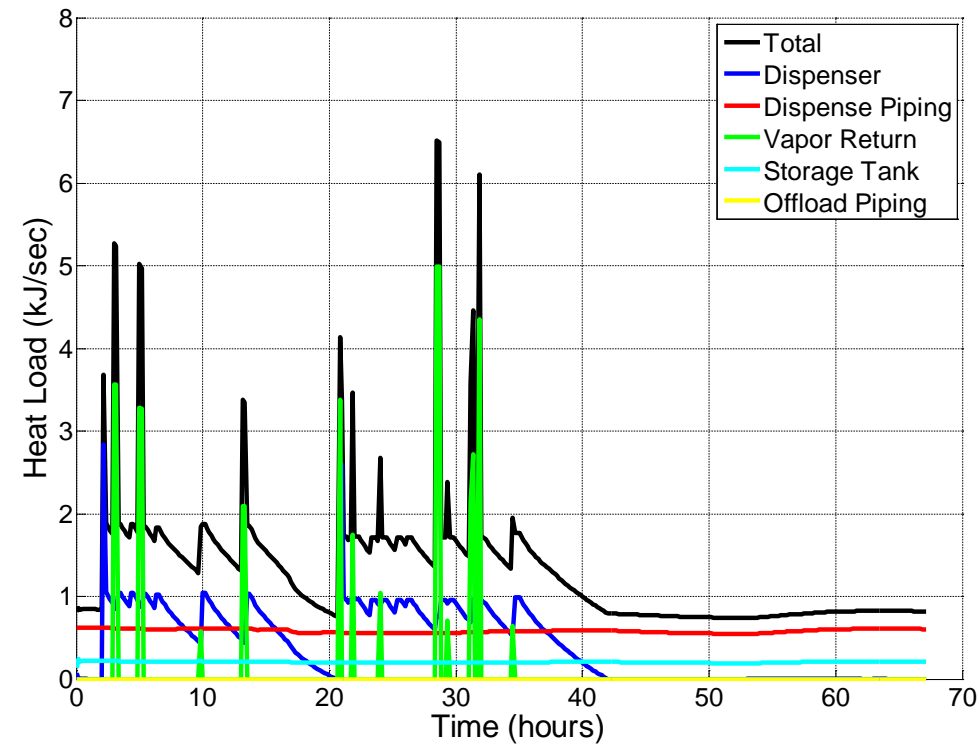

Figure C-102: Audit 2 Segment 8 Simulation Heat Transfer 

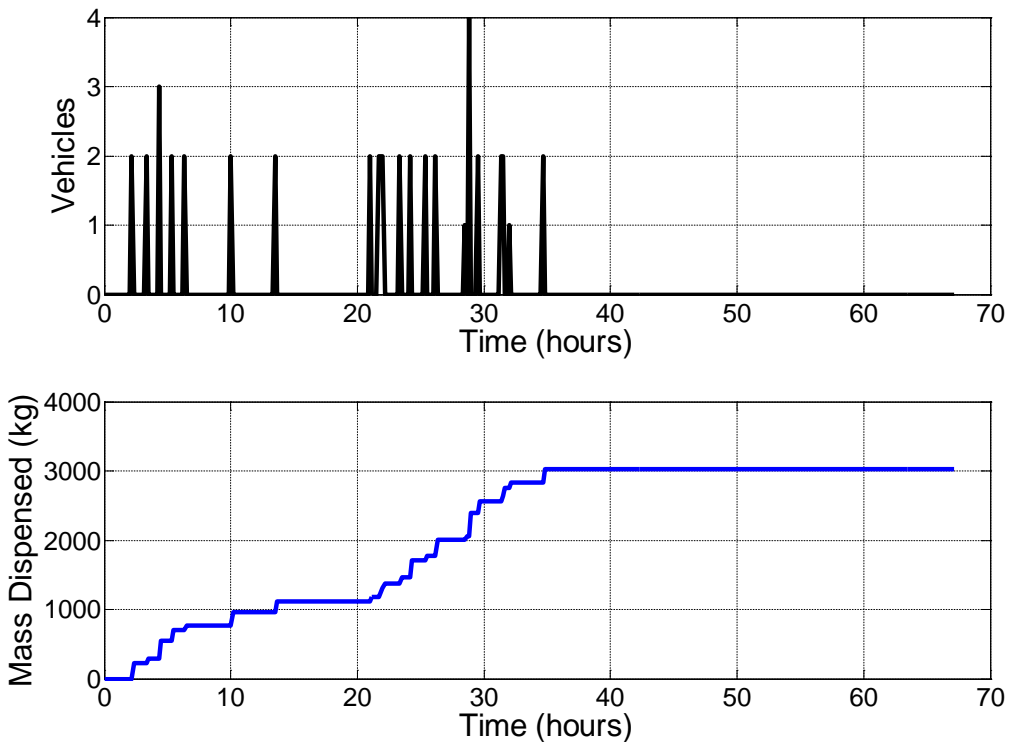

Figure C-103: Audit 2 Segment 8 Simulation Fleet Arrival Times and Mass Dispensed

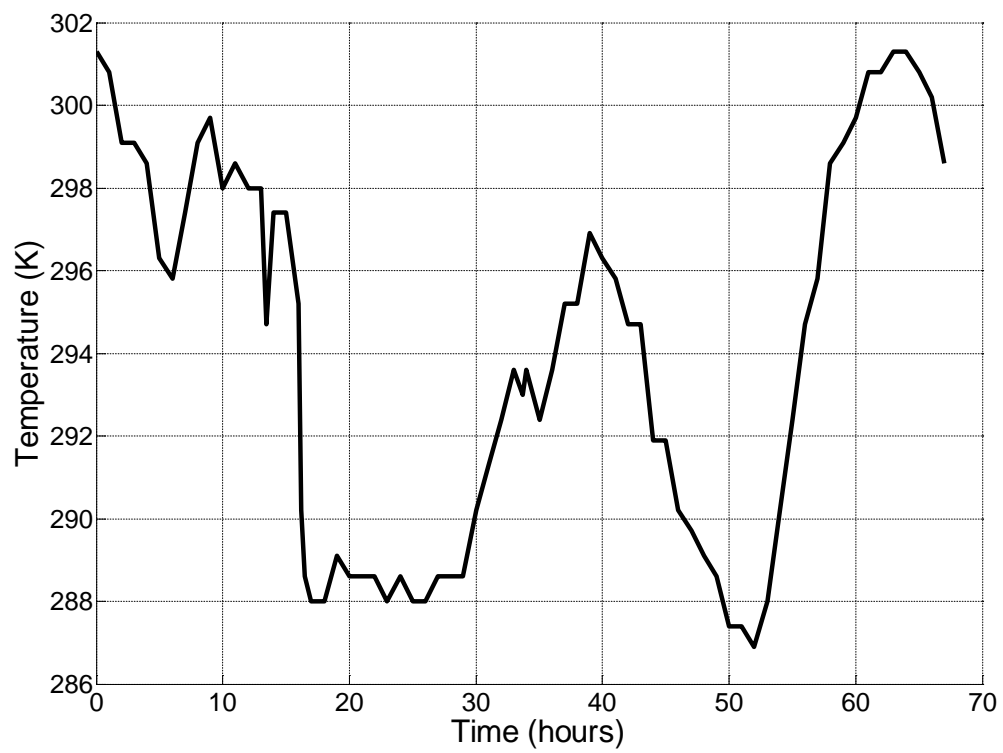

Figure C-104: Audit 2 Segment 8 Ambient Temperature 